\title{
Search for the Higgs Boson Using High-pT Isolated Like-Sign Dilepton Events in 1.96-TeV Proton-Antiproton Collisions
}

\author{
Hirokazu KOBAYASHI \\ (Doctoral Program in Physics)
}

Submitted to the Graduate School of

Pure and Applied Sciences in Partial Fulfillment of the Requirements for the Degree of Doctor of Philosophy in Science at the University of Tsukuba 


\section{Abstract}

Our physics objective is to search for the neutral Higgs boson using events containing a like-sign dilepton pair in the following reaction:

$$
q \bar{q}^{\prime} \rightarrow W^{ \pm} H \rightarrow W^{ \pm} W^{*} W^{*} \rightarrow \ell^{ \pm} \ell^{ \pm}+X
$$

The relevant Higgs boson mass region is above $160 \mathrm{GeV} / c^{2}$ for the Standard Model Higgs boson where the branching fraction of $H \rightarrow W^{*} W^{*}$ supersedes that of $H \rightarrow b \bar{b}$. The search for this signature in the region at low mass (less than $135 \mathrm{GeV} / c^{2}$ ) is, however, still important because we need to investigate various Higgs boson couplings as an essential test to convince that signals are attributed to the Higgs boson production. This channel also covers the case beyond the Standard Model that the Higgs boson couples only to the gauge bosons, which is referred to as the bosophilic or fermiophobic Higgs boson. The corresponding mass region suitable to our signature is above 110 $\mathrm{GeV} / c^{2}$ where the branching fraction of $H \rightarrow \gamma \gamma$ is overtaken by this channel. On the experimental side, the like-sign dilepton event is one of the cleanest signature in hadron collisions. This analysis exploiting such a distinctive signature is therefore expected to have a high potential of the sensitivity for the search of the Higgs boson.

The data were collected with the CDF II detector between March 2002 and September 2003, corresponding to an integrated luminosity of $193.5 \mathrm{pb}^{-1}$. At off-line, the central region $(|\eta|<1.1)$ is considered for the lepton detection. We require at least one electron with $E_{T}>20 \mathrm{GeV}$ or muon with $p_{T}>20 \mathrm{GeV} / c$ which is considered to be responsible for firing the corresponding trigger, and at least one other electron with $E_{T}>6 \mathrm{GeV}$ or muon with $p_{T}>6 \mathrm{GeV} / c$. The leptons are required to be isolated in terms of the calorimeter cone-isolation with a cone size of $R=0.4$. We require that the isolation is less than $2 \mathrm{GeV}$ for each lepton. For the events with like-sign two or more leptons that pass our selection above, we apply a cosmic-ray veto, dilepton mass cut $\left(M_{\ell \ell}>12 \mathrm{GeV} / c^{2}\right)$, and a $Z$-decay removal to clean up the sample. We also explicitly require that the leptons must be consistent with coming from the same vertex, which is an important requirement for multi-lepton signatures especially in a high-luminosity situation. The selection cuts identifies high- $p_{T}$, isolated leptons with almost "standard" identification criteria. We observed 45 like-sign dilepton events in data.

The like-sign requirement is quite effective to suppress QCD and known electroweak processes, we expect that fake-lepton backgrounds still remain at a considerable level in the events of our signature. Most of our efforts in background study focus on these fakes. The physics sources of fake electrons (including non-prompt electrons) are interactive $\pi^{ \pm}$, accidental overlap of $\pi^{0}$ 's and a track, and residual photon conversion, where the residual photon conversion is a single electron originating from the photon conversion with an unobserved partner track due to its low momentum. For the fake 
muons the sources are punch-through hadron and decay-in-fight muon. Non-interested real leptons from the semileptonic decay from heavy flavors in QCD processes are included here as one type of fake leptons. We first obtain the fake rate $R_{\text {fake }}$ which is defined as the probability for the isolated tracks to pass the lepton selection cuts, then scale, with this rate, the number of isolated like-sign tracks found in addition to the leading (trigger) lepton in the inclusive lepton samples to estimate the fake backgrounds as a lump sum. We evaluate the $R_{\text {fake }}$ using inclusive jet samples with $E_{T}>20 \mathrm{GeV}$ (JET20) and $E_{T}>50 \mathrm{GeV}$ (JET50). In this, we require a set of criteria to ensure an unbiased sample of isolated tracks in generic QCD events. We look at the inclusive jet sample with $E_{T}>100 \mathrm{GeV}$ (JET100) and inclusive high- $E_{T}(>25 \mathrm{GeV})$ photon samples for validation of the fake lepton rate. We see reasonable agreements for each sample within the estimated error.

We try to separate the contribution of residual photon conversions from the fake electron rate. Once we know the total tagging efficiency $\varepsilon_{\text {tot }}$, we can estimate the amount of the residual photon conversions from that of the tagged photon conversions by multiplying the residual ratio $R_{\text {res }}$ defined as

$$
R_{\mathrm{res}}=\frac{1-\varepsilon_{\mathrm{tot}}}{\varepsilon_{\mathrm{tot}}}, \quad \varepsilon_{\mathrm{tot}}=\frac{N_{\mathrm{tag}}}{N_{\mathrm{IDele}}},
$$

where $N_{\text {tag }}$ is the number of tagged photon conversions and $N_{\text {IDele }}$ is the number of photon conversions with one leg of electron passing our selection criteria except the photon conversion veto (seed electron). We decompose the photon conversion tagging efficiency into three parts as

$$
\varepsilon_{\mathrm{tot}}=\varepsilon_{\mathrm{track}} \cdot \varepsilon_{\mathrm{cut}} \cdot \varepsilon_{\mathrm{pt} 2.0}
$$

where $\varepsilon_{\text {track }}$ is the partner track finding efficiency and the dominant source of the inefficiency, $\varepsilon_{\text {cut }}$ is the efficiency of the criteria applied to a track pair to identify a photon conversion (tagging cuts), $\varepsilon_{\mathrm{pt} 2.0}$ is the partner track finding efficiency in the region $p_{T}>2 \mathrm{GeV} / c$. We use Data and Monte Carlo sample to obtain the kinematic information of the photon conversion. We calculate the overall photon conversion tagging efficiency $\varepsilon_{\text {tot }}$ defined in Eq.(2) and the residual ratio $R_{\text {res }}$ of Eq.(1). We see that $\varepsilon_{\text {tot }}$ increases from $50 \%$ to $60 \%$ as the conversion electron $E_{T}$ increases.

We compare the expected like-sign dilepton events with the observed ones for the inclusive high- $p_{T}$ lepton sample. We look into the second lepton in terms of its transverse momentum $p_{T}$, event $\eta$, and calorimeter isolation. The major background components of the like-sign dilepton events are the fake leptons and the residual photon conversions with the latter being dominant at high- $p_{T}$ region. The contribution of the background estimated by the Monte Carlo simulation ( $W Z, Z Z, t \bar{t}$ and $W$ plus heavy flavors) is found to be small. We also look into the event topological variables such as $\mathbb{E}_{T}$, lepton-pair azimuthal opening-angle, and vector sum of lepton's $p_{T}$ for the like-sign 
dilepton events. The observed number of events in the data is well saturated with our background expectation, and we see reasonable agreements within the statistical errors of the data.

We determine a signal region in the plane of the second lepton $p_{T}\left(p_{T 2}\right)$ and the vector sum of $p_{T}$ 's of the two leptons $\left(p_{T 12}\right)$ to enhance the signal significance $(S / \sqrt{B})$. We use $160 \mathrm{GeV} / c^{2}$ Standard Model Higgs boson Monte Carlo events as signal samples and the total number of expected backgrounds, then look for the region to improve the signal significance as much as reasonable to the extent that we can expect at least about one signal-event with the base-projection of the integrated luminosity in Run II which is $4.4 \mathrm{fb}^{-1}$. The cut is chosen to be $(18,35) \mathrm{GeV} / \mathrm{c}$ for the Higgs boson mass equal or more than $160 \mathrm{GeV} / c^{2}$. We divide the $p_{T 2}$ versus $p_{T 12}$ plane into four regions: $\mathrm{A}\left(p_{T 2}>18 \mathrm{GeV} / c\right.$ and $\left.p_{T 12} \leq 35 \mathrm{GeV} / c\right), \mathrm{B}\left(p_{T 2} \leq 18 \mathrm{GeV} / c\right.$ and $\left.p_{T 12} \leq 35 \mathrm{GeV} / c\right)$, $\mathrm{C}\left(p_{T 2} \leq 18 \mathrm{GeV} / c\right.$ and $\left.p_{T 12}>35 \mathrm{GeV} / c\right)$, and the signal region $\left(p_{T 2}>18 \mathrm{GeV} / c\right.$ and $\left.p_{T 12}>35 \mathrm{GeV} / c\right)$. We compare the number of expected background events with that of observed ones in the control regions $(\mathrm{A}-\mathrm{C})$ as a sanity test. In each control region we see a reasonable agreement, while we observe no event in the signal region.

Since we observed no event in the signal region of the second lepton $p_{T}$ greater than $18 \mathrm{GeV} / c$ and the vector sum of $p_{T}$ 's of the two leptons greater than $35 \mathrm{GeV} / c$, we set the upper limit on the production cross section times the branching fraction $\sigma(W H) \times B_{r}(H \rightarrow W W)$. The upper limit was obtained to be $12 \mathrm{pb}$ at the $95 \%$ C.L. for the Higgs boson mass of $110 \mathrm{GeV} / c^{2}$ and $8 \mathrm{pb}$ for $160 \mathrm{GeV} / c^{2}$. 


\section{Acknowledgments}

I would like to thank my thesis adviser Prof. Koji Takikawa and Prof. Shinhong Kim for his guidance through my graduate school. You gave a great opportunity to study physics at CDF Run II experiment and financial supports staying at Fermilab. I would like to thank Prof. Yoshihiro Seiya for your many thoughtful advices. You patiently tried to have a correct understanding of my analysis and gave many suggestions from your empirical knowledge derived from CDF experiment. I would like to thank Dr. Kazuhiro Yamamoto for your advice from an objective perspective and technical supports during staying at Osaka City University. Prof. John Conway, Prof. Weiming Yao, Prof. Tom Junk, and Prof. Aaron Dominguez who are the conveners of Higgs Working group, suggested a lot of fruitful comments for my analysis. Prof. Stephan Lammel and Dr. Beate Heinemann , who are the conveners of Exotic physics working group, organized my analysis and gave me a chance of presentation at the CDF Collaboration meeting. I enjoyed a precious experience.

Of course, experimental particle physics is no longer product for one person, but supported by hundreds of people. I would like to thank all of the people who have designed and constructed the Tevatron and CDF detector, took the data, wrote the analysis code, and so on.

I would like to thank stuff members of the University of Tsukuba, Prof. Fumihiko Ukegawa, Kazuhiko Hara, Takasumi Maruyama, Hiroyuki Matsunaga Yuji Takeuchi. They provided me the opportunity to work at the CDF experiments. And also I would like to thank CDF students, Satoru Uozumi, Koji Sato, Hideaki Takano, Takashi Ogawa, Tomohiro Yamashita, Naho Tanimoto, Takashi Akimoto, Yoshio Ishizawa, Masato Aoki, and Vivek Tiwari, who shared the two year's life at Fermilab with me.

I would like to thank stuff members of the Osaka-City University, Prof. Toru Okusawa, Yukihiro Kato, Hirotoshi Toyoda. You welcome me to Osaka-City University and gave a position of research student during about one year. I owe you more than I can repay. Thank you.

I would like to thank my family. Yujiro, Fukuko, and Mitsuko, you provided me with all of the warmth, support, and encouragement I could have ever wanted and more. Thank you very much.

Finally I thank the Fermilab staff and the technical staffs of the participating institutions for their vital contributions. This work was supported by the U.S. Department of Energy and National Science Foundation; the Italian Istituto Nazionale di Fisica Nucleare; the Ministry of Education, Culture, Sports, Science and Technology of Japan; the Natural Sciences and Engineering Research Council of Canada; the National Science Council of the Republic of China; the Swiss National Science Foundation; the A.P. Sloan Foundation; the Bundesministerium fuer Bildung und Forschung, Germany; the Korean Science and Engineering Foundation and the Korean Research Foundation; the Particle Physics and Astronomy Research Council and the Royal Society, UK; the 
Russian Foundation for Basic Research; the Comision Interministerial de Ciencia y Tecnologia, Spain; and in part by the European Community's Human Potential Programme under contract HPRN-CT-20002, Probe for New Physics. 


\section{Contents}

1 Introduction 1

1.1 Standard Model . . . . . . . . . . . . . . . . . . . . . . . 2

1.1.1 Standard Model Elements . . . . . . . . . . . . . . . . . 2

1.1.2 Standard Model Structure . . . . . . . . . . . . . . . . . 3

1.1.3 Quantum Electrodynamics: $\mathrm{U}(1)_{Q} \ldots \ldots \ldots \ldots \ldots$

1.1.4 Quantum Chromodynamics: $\mathrm{SU}(3)_{C} \ldots \ldots \ldots \ldots$

1.1.5 Electroweak Theory: $\mathrm{SU}(2)_{L} \otimes \mathrm{U}(1)_{Y} \ldots \ldots \ldots \ldots \ldots$

1.1.6 Electroweak Symmetry Breaking . . . . . . . . . . . . . 8

1.1 .7 Renormalizability . . . . . . . . . . . . . . . . 10

1.1.8 Input to the Standard Model . . . . . . . . . . . . . . . . 11

1.2 An Energy Scale $\Lambda$ when the Standard Model breaks down . . . . . . . 11

1.3 Present Status of the SM Higgs Boson Search . . . . . . . . . . . . . 12

1.4 The Standard Model Higgs Boson Search . . . . . . . . . . . . . . . . . 14

1.4.1 Standard Model Higgs Boson Decay Modes . . . . . . . . . . . . 15

1.4.2 Standard Model Higgs Boson Production at Tevatron . . . . . . 16

1.5 "Bosophilic" Higgs Boson . . . . . . . . . . . . . . . . . . . . . . 18

1.6 Physics Motivation . . . . . . . . . . . . . . . . . . . . 21

2 Apparatus 23

2.1 Fermilab Run II Accelerator Complex . . . . . . . . . . . . . . . . . . . . 23

2.1 .1 Proton Beam . . . . . . . . . . . . . . . . . 23

2.1 .2 Antiproton Beam . . . . . . . . . . . . . . . . . . 24

2.1 .3 Colliding Beams . . . . . . . . . . . . . . . . 25

2.2 Collider Detector at Fermilab . . . . . . . . . . . . . . . . 27

2.2 .1 Silicon Tracking Systems . . . . . . . . . . . . . . . . 29

2.2 .2 Central Outer Tracker . . . . . . . . . . . . . . . . . 33

2.2 .3 Magnetic Field . . . . . . . . . . . . . . . . . . . . . 33

2.2 .4 Calorimetry . . . . . . . . . . . . . . . . . . . . 35

2.2 .5 Muon Systems . . . . . . . . . . . . . . . . . . . . . . 40

2.2 .6 Trigger Systems . . . . . . . . . . . . . . . . . . . . . 40

2.2.7 Event Generation and Simulation . . . . . . . . . . . . . 45 
2.2 .8 Object-oriented Software . . . . . . . . . . . . . . 46

3 Base-Line Selection of Like-Sign Dilepton Events 47

3.1 Physics Objects at CDF . . . . . . . . . . . . . . . 47

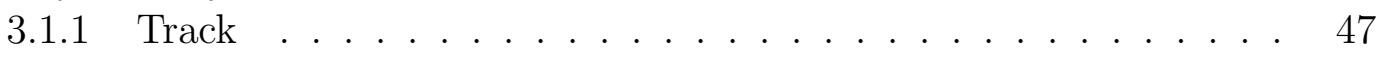

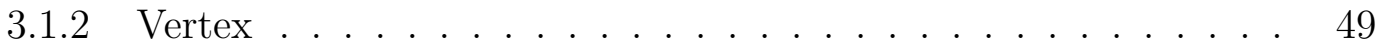

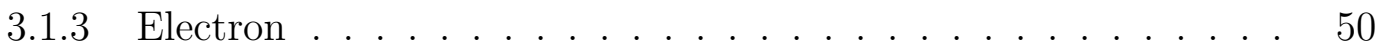

3.1 .4 Muon ......................... 62

3.2 Triggers and Datasets . . . . . . . . . . . . . . . . 70

3.2.1 Good Runs and Integrated Luminosity . . . . . . . . . . . . . . 71

3.2.2 Inclusive High $p_{T}$ Lepton samples . . . . . . . . . . . . . . . . 71

3.3 Selection Cuts . . . . . . . . . . . . . . . . . . . . 74

3.4 Survived Events . . . . . . . . . . . . . . . . . . . . . . . . . . . . . . . . . . . . . . . . . . .

3.5 Summary of Event Selection . . . . . . . . . . . . . . . 75

4 Background Estimation for High- $p_{T}$ Like-Sign Dilepton $\quad 81$

4.1 Monte Carlo Background Samples . . . . . . . . . . . . . . . . 81

4.2 Fake Lepton Background . . . . . . . . . . . . . . . . . . . . . . . . 82

4.2.1 Lepton Fake-Rate Measurement . . . . . . . . . . . . . . . . . 83

4.2.2 Validation of the Lepton Fake Rate . . . . . . . . . . . . . . . 84

4.3 Residual Photon Conversion Background . . . . . . . . . . . . . . . . . 95

4.3.1 Conversion Flag Rate . . . . . . . . . . . . . . . . . . . . 95

4.3.2 Photon Conversion Tagging Efficiency . . . . . . . . . . . . . . . . 98

4.3.3 Photon Conversion Monte Carlo Sample . . . . . . . . . . . . . 98

4.3.4 Partner Track Finding Efficiency . . . . . . . . . . . . . . . . . 99

4.3.5 Conversion Tagging Cut Efficiency . . . . . . . . . . . . . . 105

4.3.6 Partner Track Finding Efficiency above $2 \mathrm{GeV} / c$. . . . . . . . 108

4.3.7 Overall Efficiency and Residual Conversion Ratio . . . . . . . . 110

4.4 Corrected Electron Fake Rate . . . . . . . . . . . . . . . . . . . . 112

4.5 Expected Event to Data Comparison . . . . . . . . . . . . . . . . 116

4.5.1 2nd Leg Lepton . . . . . . . . . . . . . . . . . . . . . . . . 116

4.5.2 Event Topological Variables . . . . . . . . . . . . . . 116

4.6 Summary of Background Estimation . . . . . . . . . . . . . . . . 123

5 Topological Cut $\quad 125$

5.1 Determination of Signal Region . . . . . . . . . . . . . . . 125

5.2 Checks around the Signal Box . . . . . . . . . . . . . . . . 126

6 Signal Acceptance 133

6.1 Signal Monte Carlo Samples . . . . . . . . . . . . . . . . . . 133

6.2 Geometrical and Kinematical Acceptance . . . . . . . . . . . . . . . . . 134 
6.3 Isolation Cut Efficiency . . . . . . . . . . . . . . . . . . . . . . . . . . 139

6.4 Lepton Identification Efficiency . . . . . . . . . . . . . . . . 146

6.5 Dilepton Selection Efficiency . . . . . . . . . . . . . . . . . . . . 146

6.6 Topological Cut Efficiency . . . . . . . . . . . . . . . . . . . . . . 153

6.7 Trigger Efficiency and Other Scale Factors . . . . . . . . . . . . . 153

6.7.1 High $p_{T}$ Electron Trigger Efficiency . . . . . . . . . . 153

6.7.2 High $p_{T}$ Muon Trigger Efficiency . . . . . . . . . . . . . . 154

6.7.3 Event Weight for the Difference of Integrated Luminosity . . . . 155

6.8 Total Detection Efficiency . . . . . . . . . . . . . . . . . 156

6.9 Systematic Uncertainties . . . . . . . . . . . . . . . . . . . 156

7 Results and Conclusions 161

7.1 Pseudo Experiments . . . . . . . . . . . . . . . . . . . . . 161

7.2 Observed 95\% C.L. Cross Section Upper Limit . . . . . . . . . . . . . 161

7.3 The Other Direct SM Higgs Boson Search in CDF Run II . . . . . . . . 162

7.4 Conclusions . . . . . . . . . . . . . . . . . . . . . . . . . . 162 


\section{List of Figures}

1.1 The Higgs potential $V$ for a complex scalar field with $\mu^{2}<0$ and $\lambda>0.9$

1.2 The lower [15] and the upper [14] Higgs boson mass bounds as a function of the energy scale $\lambda$ at which the Standard Model breaks down, assuming $M_{t o p}=175 \mathrm{GeV} / c^{2}$ and $\alpha_{s}\left(M_{Z}\right)=0.118$. The shaded areas above reflect the theoretical uncertainties in the calculations of the Higgs boson mass bounds. This figure is taken from [16] . . . . . . . . . . . 13

1.3 Standard Model Higgs boson interactions at tree-level . . . . . . . . . . 14

1.4 Branching ratios of the dominant decay modes of the Standard Model Higgs boson [17]. These results have been obtained with the program HDECAY [19], and include QCD corrections beyond the leading order.

1.5 Higgs boson production cross-section (in units of pb) at the Tevatron for the various production mechanisms as a function of the Higgs boson mass, taken from refs [17]. The full NLO QCD-corrected results are shown for the gluon fusion $g g \rightarrow H$, vector boson fusion $q q \rightarrow$ $V^{*} V^{*} q q \rightarrow H q q$, Higgs-strahlung processes $q \bar{q} \rightarrow V^{*} \rightarrow V H$. Treelevel cross-section are exhibited for Higgs boson production processes in association with heavy quarks pairs: $g g, q \bar{q} \rightarrow t \bar{t} H . . . . . . .$.

1.6 (a) Two-photon decay of a bosophilic Higgs boson via a $W$ boson loop; (b) decay of a bosophilic Higgs boson to $b \bar{b}$ via virtual $W$ and $Z$ bosons. The latter decay mode is suppressed relative to the former by $m_{b}^{2} / M_{W}^{2}$.

1.7 Branching ratios of a bosophilic Higgs boson decay to $\gamma \gamma, W W^{*}, Z Z^{*}$, and $\gamma Z$, versus the Higgs boson mass. . . . . . . . . . . . . . . . 20

2.1 Pictorial overview of the Fermilab accelerator complex for Run II. . . . 24

2.2 Initial instantaneous luminosity for each store. . . . . . . . . . . . . . 26

2.3 Integrated luminosity. Upper line is the delivered luminosity and lower one is the received luminosity. . . . . . . . . . . . . . . 26

2.4 Longitudinal view of half of the CDF Run II detector. . . . . . . . . . . 28

2.5 Longitudinal view of the CDF tracking system, representing a quarter of the whole detector. . . . . . . . . . . . . . . 28

2.6 Coordinate system used at CDF . . . . . . . . . . . . . . . . . 29 
2.7 Upper figure is a view of the three barrels of SVX II silicon detector. Lower one is a view of one barrel. One barrel has 12 wedges and 5 layers. 31

2.8 The CDF Silicon detectors (Layer 00 (Pink), SVX II(Blue), and ISL(Red)) end view showing the layering and space frame used to mount the detector. 32

2.9 Upper figure is the endplate slots. In this figure the odd are stereo and the even are axial superlayers. Lower figure is a single cell layout. . . .

2.10 Top: Three-dimensional view of a central calorimeter wedge showing the location of each tower in pseudorapidity. Bottom: Illustration of the CES response to an EM shower. . . . . . . . . . . . . . . 36

$2.11 r-z$ cross-section view of the upgraded Endplug Calorimeter of CDF.

2.12 Tower geometry for the CDF run II Endplug calorimeters. Each wedge covers 15 degree in azimuth. As shown, towers $0-14$ span the region $1.1<|\eta|<2.1$, and towers $16-22$ span the region $2.1<|\eta|<3.6$. . . .

2.13 Schematic drawing of a 45 degree section of the PES detector. The PES is calibrated using a radioactive source, which traverses the indicated path during calibration and is retracted into a shielded box when not in use. . . . . . . . . . . . . . . . . . .

2.14 Cross-section view of drift tubes in a muon chamber. A stub is formed from the trajectory of a muon passing through the chamber, and the stub is matched to a track to become a reconstructed muon. . . . . . . 41

2.15 Map of the muon coverage for this analysis. . . . . . . . . . . . . 42

2.16 The three level deadtime-less trigger used to control DAQ of the CDF

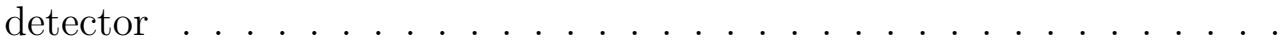

2.17 The readout functional block diagram. The acronyms have been already introduced in the text, apart from the following: XFT: Extremely fast tracker, XTRP: Track extrapolation module, SVT: SVX track processor in Level2 trigger system, TSI: Trigger System Interface. . . . . . . . . .

3.1 An illustration of the tracking parameters: the figure on the left shows a charged track in the $r-z$ view of the tracking volume. The figure on the right shows an $r-\phi$ view of the track. $d_{0}$ is the track's impact parameter, or point of closest approach to the origin. $C$ is the curvature. 48

3.2 Left distributions are number of vertices in inclusive high $p_{T}$ lepton samples. Right one are primary vertex z position. Solid lines are electron sample and broken ones are muon sample. We require at least one tight lepton for each event. . . . . . . . . . . . . . . . . .

3.3 CEM tower gains. A global correction factor of 1.015 is applied in order to move the mean back to what it was before the corrections. This prevents these corrections from changing the absolute energy scale. . .

$3.4 E / p$ as a function of run number. The average $E / p$ is calculated in the range between 0.9 and $1.1 \ldots \ldots \ldots \ldots \ldots$ 
3.5 Left plot is the CEM energy correction factor applied to the data. Right one is $E / p$ as a function of CES $x$ after the corrections. . . . . . . .

3.6 The electron variables used for the selection of the events. The tight electron in the $Z \rightarrow e^{+} e^{-}$candidates events (dots) and in the Monte Carlo (solid histogram) are used. For each variable all the selection criteria, but the one including the variable itself, are applied. The number of Monte Carlo events is normalized to the $193.5 \mathrm{fb}^{-1}$ data. Upper plots are electron $E_{T}$ and $p_{T}$ distributions and lower ones are detector $\eta$ and azimuthal angle $\phi$ of EM cluster from $Z \rightarrow e^{+} e^{-}$candidate events. . . .

3.7 The electron variables used for the selection of the events. The tight electron in the $Z \rightarrow e^{+} e^{-}$candidates events (dots) and in the Monte Carlo (solid histogram) are used. For each variable all the selection criteria, but the one including the variable itself, are applied. The number of Monte Carlo events is normalized to the $193.5 \mathrm{fb}^{-1}$ data. Upper plots are calorimeter isolation $I S O_{0.4}^{\text {cal }}$ and $H A D / E M$ distributions. Lower plots are $L_{\mathrm{shr}}$ and $E / p$ distributions . . . . . . . . . . . .

3.8 The electron variables used for the selection of the events. The tight electron in the $Z \rightarrow e^{+} e^{-}$candidates events (dots) and in the Monte Carlo (solid histogram) are used. For each variable all the selection criteria, but the one including the variable itself, are applied. The number of Monte Carlo events is normalized to the $193.5 \mathrm{fb}^{-1}$ data. Upper plots are CES strip $\chi^{2}$ and $\Delta x \times Q$ distributions. Middle plots are $\Delta z$ and impact parameter $d_{0}$ distributions. Lower plot is $z_{0}-z_{v t x}$ distribution.

3.9 Upper plot is a distribution of the conversion point radius from detector center. Lower plots are $\delta_{x y}$ and $\Delta(\cot \theta)$ distributions. These conversions are selected from $4 \mathrm{GeV}$ conversion sample by $|\Delta(\cot \theta)|<$

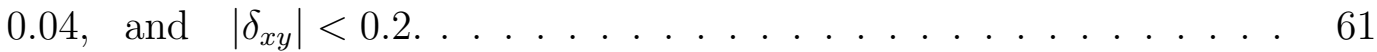

3.10 Passage of a muon through the CDF detector. . . . . . . . . . . . . 62

3.11 The COT exit radius $\rho$ for CMX $W \rightarrow \mu \nu$ candidate events. The points are the data and the yellow histogram is the simulation. The simulation is normalized to the number of data events in the range $150<\rho<280$ $\mathrm{cm}$. The lack of CMX events at low $\rho$ is a reflection of the XFT trigger acceptance due to the requirement of hits on all 4 axial superlayers. A cut of $\rho<140 \mathrm{~cm}$ is chosen to remove this inefficient region. . . . . . .

3.12 The muon variables used for the selection of the events. The tight muon in the $Z \rightarrow \mu^{+} \mu^{-}$candidates events (dots) and in the Monte Carlo (solid histogram) are used. For each variable all the selection criteria, but the one including the variable itself, are applied. The number of Monte Carlo events is normalized to the $193.5 \mathrm{fb}^{-1}$ data. Upper plots are CMUP and CMX muon's $p_{T}$ and $I S O_{0.4}^{\text {cal }}$ distributions and lower ones are event $\eta$ and azimuthal angle $\phi$ of track. . . . . . . . . . . . . . 
3.13 The muon variables used for the selection of the events. The tight muon in the $Z \rightarrow \mu^{+} \mu^{-}$candidates events (dots) and in the Monte Carlo (solid histogram) are used. For each variable all the selection criteria, but the one including the variable itself, are applied. The number of Monte Carlo events is normalized to the $193.5 \mathrm{fb}^{-1}$ data. Upper plots are muon electromagnetic calorimeter energy deposition distributions for CMUP and CMX muons. Lower plots are muon hadron calorimeter energy deposition distributions for CMUP and CMX muons. . . . . . .

3.14 The muon variables used for the selection of the events. The tight muon in the $Z \rightarrow \mu^{+} \mu^{-}$candidates events (dots) and in the Monte Carlo (solid histogram) are used. For each variable all the selection criteria, but the one including the variable itself, are applied. The number of Monte Carlo events is normalized to the $193.5 \mathrm{fb}^{-1}$ data. Upper and middle plots are track-stub matching $\Delta x=r \times \Delta \phi$ for CMUP and CMX muons. Lower plots are impact parameter $d_{0}$ and $z_{0}-z_{v t x}$ distributions. . . . .

3.15 Upper distributions are transverse energy for all electrons in the inclusive high $p_{T}$ electron sample. Lower distributions are transverse momentum for all muons in the inclusive high $p_{T}$ muon sample. . . . . . . . . . . .

3.16 Distributions of $p_{T}$, event $\eta$, and $I S O_{0.4}^{\text {cal }}$ for the 2 nd lepton leg in the like-sign dilepton events selected from inclusive high- $p_{T}$ lepton data. . .

3.17 Distributions of $\mathbb{E}_{T}, \cos \phi_{12}$, and $p_{T 12}$ for the like-sign dilepton events selected from inclusive high- $p_{T}$ lepton data. . . . . . . . . . .

4.1 Track $p_{T}$ distributions of isolated tracks (upper left), electrons (lower left), and muons (lower right) in the JET samples. Points correspond to JET20 and JET50 samples. . . . . . . . . . . . . . . . . . . .

4.2 Cone 0.4 calorimeter isolation $I S O_{0.4}^{c a l}$ distributions of isolated tracks (upper left), electrons (lower left), and muons (lower right) in the JET samples. Points correspond to JET20 and JET50 samples. . . . . . . .

4.3 Track $p_{T}$ dependence of the lepton fake rate. The upper left (right) plot shows the electron (muon) fake rate for the JET20 and JET50 samples. The lower left (right) plot shows the combined electron (muon) fake rate. 87

4.4 Cone 0.4 calorimeter isolation $I S O_{0.4}^{c a l}$ dependence of the lepton fake rate. The upper left (right) plot shows the electron (muon) fake rate for the JET20 and JET50 samples. The lower left (right) plot shows the combined electron (muon) fake rate. . . . . . . . . . . .

4.5 Upper plot is $\cos \phi_{12}$ distribution, where $\phi_{12}$ is $\phi$ separation of isolated track and trigger jet. The points are JET20 and JET50 samples. We define that $\cos \phi_{12}>-0.8$ region is no-bias region and $\cos \phi_{12}<-0.8$ region is bias region. . . . . . . . . . . . . . . . 
4.6 Electron and muon $p_{T}$ dependence of fake rate. Upper plots are fake rates in the no-bias region $\left(\cos \left(\phi_{12}\right)>-0.8\right)$. Middle plots are fake rates in the bias region $\left(\cos \left(\phi_{12}\right)<-0.8\right)$. Lower plots are combined fake rates for each region. We take the half of difference between no-bias fake rate and bias one as a systematic error. . . . . . . . . . . . .

4.7 Electron and muon $I S O_{0.4}^{c a l}$ dependence of fake rate. Upper plots are fake rates in the no-bias region $\left(\cos \left(\phi_{12}\right)>-0.8\right)$. Middle plots are fake rates in the bias region $\left(\cos \left(\phi_{12}\right)<-0.8\right)$. Lower plots are combined fake rates for each region. We take the half of difference between no-bias fake rate and bias one as a systematic error. . . . . . . . . . . . . .

4.8 Expected and observed lepton $p_{T}$ and $I S O_{0.4}^{c a l}$ distributions in the JET70 sample. The upper left (right) plot shows the electron (muon) $p_{T}$ distribution and the lower left (right) plot shows the $I S O_{0.4}^{c a l}$ distribution. The filled regions represent the number of expected events with the estimated errors, and the points represent the number of observed events. . . .

4.9 Expected and observed lepton $p_{T}$ and $I S O_{0.4}^{c a l}$ distributions in the JET100 sample. The upper left (right) plot shows the electron (muon) $p_{T}$ distribution and the lower left (right) plot shows the $I S O_{0.4}^{c a l}$ distribution. The filled regions represent the number of expected events with the estimated errors, and the points represent the number of observed events. . . .

4.10 Expected and observed lepton $p_{T}$ and $I S O_{0.4}^{c a l}$ distributions in the inclusive high- $E_{T}$ photon sample. The upper left (right) plot shows the electron (muon) $p_{T}$ distribution and the lower left (right) plot shows the $I S O_{0.4}^{c a l}$ distribution. The filled regions represent the number of expected events with the estimated error, and points represent the number of observed events. . . . . . . . . . . . . . . . . . .

4.11 The theoretical calculation of the relative probability of energy sharing in the photon conversion as a function of the fractional electron energy $[76] . \ldots \ldots \ldots \ldots \ldots \ldots$

4.12 Conversion flag rate defined in Equation 4.2. A closed circle and a closed square correspond to the JET + electron $\left(E_{T}>6 \mathrm{GeV}\right)$ events in the JET20 and JET50 samples. An open circle and a open square correspond to the 2 nd leg electrons $\left(E_{T}>6 \mathrm{GeV}\right)$ of the like-sign ee or $\mu e$ events in the inclusive high- $p_{T}$ electron and muon samples. . . . . .

4.13 Distribution of the radius of the conversion points from the detector center (left), and the fractional electron energy (right). The points correspond to data and the histograms correspond to the Monte Carlo simulation. . . . . . . . . . . . . . . . 
4.14 Conversion electron $p_{T}$ spectra for $E_{T}>6 \mathrm{GeV}$ (top left), $E_{T}>10 \mathrm{GeV}$ (bottom left), and $E_{T}>15 \mathrm{GeV}$ (bottom right). The points correspond to data and the histograms correspond to the reconstructed Monte Carlo simulation. The Monte Carlo sample is normalized to data in the region of the partner track $p_{T}>2 \mathrm{GeV} / c \ldots \ldots \ldots$

4.15 Conversion partner track $p_{T}$ spectra for $E_{T}>6 \mathrm{GeV}$ (top left), $E_{T}>$ $10 \mathrm{GeV}$ (bottom left), and $E_{T}>15 \mathrm{GeV}$ (bottom right) of the seed electron. The points correspond to data and the histograms correspond to the $p_{T}$ distribution at the Monte Carlo OBSP level. The Monte Carlo sample is normalized to data in the region of the partner track $p_{T}>2$ $\mathrm{GeV} / c \ldots \ldots \ldots \ldots \ldots \ldots$

4.16 The conversion electron $p_{T}$ spectrum (left), and the partner track $p_{T}$ spectrum (right). The points correspond to data and the histograms correspond to the Monte Carlo simulation. The solid line histogram shows the spectrum which is reconstructed after the detector simulation, while the broken line histogram shows the spectrum at the OBSP level. The Monte Carlo spectra are normalized to data in the region of the partner track $p_{T}$ above $2 \mathrm{GeV} / c \ldots \ldots \ldots$

4.17 The partner track $p_{T}$ dependence of the partner track finding efficiency. We have normalized the Monte Carlo distribution to data in the region above $2 \mathrm{GeV} / c \ldots \ldots \ldots \ldots$

4.18 The conversion electron $E_{T}$ dependence of the partner track finding efficiency. We have normalized the Monte Carlo distribution to data in the region of the partner track $p_{T}$ above $2 \mathrm{GeV} / c$. . . . . . . . . . . . 104

4.19 The illustrations of the conversion tagging by means of the CES strip hit information.

4.20 The $z$ position difference between the electron passing the identification cuts and the highest energy CES cluster in the nearest three towers for each $\phi$ side (right side and wrong side). The upper plot shows data and the lower plot shows the Monte Carlo simulation. . . . . . . . . . . . . 106

4.21 The difference of the electron-positron $z$ coordinates on the CES plane for the pairs passing and failing the conversion tagging cuts. The electron $E_{T}$ cut is applied at $8 \mathrm{GeV}$. . . . . . . . . . . . . . 107

4.22 The difference of the electron-positron $z$ coordinates on the CES plane for cases of "no track found" and "track found". The electron $E_{T}$ cut is

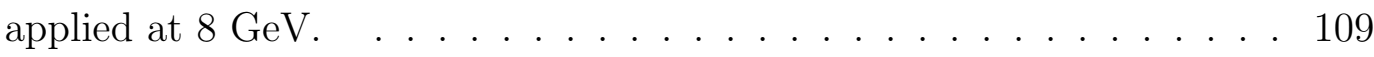

4.23 The electron $E_{T}$ dependence of the photon conversion tagging efficiency. 110

4.24 The electron $E_{T}$ dependence of the residual photon conversion ratio. . 111

4.25 The conversion electron $p_{T}$ and $I S O_{0.4}^{c a l}$ dependence of the conversion flag rate for the JET20 and JET50 samples. 
4.26 The conversion electron $p_{T}$ and $I S O_{0.4}^{\text {cal }}$ dependence of the residual conversion ratio for the JET20 and JET50 samples.

4.27 The $p_{T}$ and $I S O_{0.4}^{c a l}$ dependence of the original and corrected electron fake rates. The fake rates are combined values between the JET20 and JET50 samples.

4.28 Expected and observed second leg $p_{T}$ (top left), event $\eta$ (bottom left), and $I S O_{0.4}^{c a l}$ (bottom right) spectra for the inclusive high- $p_{T}$ trigger sample

4.29 Expected and observed second leg $p_{T}$ (top left), event $\eta$ (bottom left), and $I S O_{0.4}^{\text {cal }}$ (bottom right) spectra for the lepton+track trigger sample.

4.30 Expected and observed second leg $p_{T}$ (top left), event $\eta$ (bottom left), and $I S O_{0.4}^{\text {cal }}$ (bottom right) spectra for the inclusive low- $p_{T}$ trigger sample.

4.31 Expected and observed missing transverse $E_{T}$ (top left), lepton-pair opening angle (bottom left), and vector sum of lepton $P_{\mathrm{T} 12}$ (bottom right) for the like-sign dilepton events in the inclusive high- $p_{T}$ trigger

4.32 Expected and observed missing transverse $E_{T}$ (top left), lepton-pair opening angle (bottom left), and vector sum of lepton $P_{\mathrm{T} 12}$ (bottom right) for the like-sign dilepton events in the lepton+track data. . . . .

4.33 Expected and observed missing transverse $E_{T}, \cos \phi_{12}$ and $P_{\mathrm{T} 12}$ for inclusive low- $p_{T}$ data.

$5.1 p_{T 2}$ vs $p_{T 12}$ scatter plots of the signal and backgrounds. . . . . . . . 128

5.2 Upper (Lower) plot shows the $S / \sqrt{B}$ as a function of $p_{T 12}$ cut value for the $110 \mathrm{GeV} / c^{2}$ bosophilic Higgs $\left(160 \mathrm{GeV} / c^{2}\right.$ SM Higgs). . . . . . . .

5.3 Upper (Lower) plot shows the number of expected signal events as a function of $p_{T 12}$ cut value for the $110 \mathrm{GeV} / c^{2}$ bosophilic Higgs (160 $\left.\mathrm{GeV} / c^{2} \mathrm{SM} \mathrm{Higgs}\right) . \ldots \ldots \ldots \ldots \ldots$

$5.4 p_{T 2}$ vs $p_{T 12}$ scatter plot. The region $\mathrm{A}$ is defined by $p_{T 2}>18 \mathrm{GeV} / c$ and $p_{T 12} \leq 35 \mathrm{GeV} / c$. The region $\mathrm{B}$ is defined by $p_{T 2} \leq 18 \mathrm{GeV} / c$ and $p_{T 12} \leq 35 \mathrm{GeV} / c$. The region $\mathrm{C}$ is defined by $p_{T 2} \leq 18 \mathrm{GeV} / c$ and $p_{T 12}$ $>35 \mathrm{GeV} / c$. The signal box is defined by $p_{T 2}>18 \mathrm{GeV} / c$ and $p_{T 12}$ $>35 \mathrm{GeV} / c$. Closed (Open) points correspond to the inclusive high $p_{T}$ electron (muon) sample. The differentiation between $e \mu$ and $\mu e$ is meant to indicate the first one is the leading lepton.

6.1 Upper (Lower) plot shows the standard model (bosophilic) Higgs boson branching ratio. . . . . . . . . . . . . . . . . . 135 
6.2 Signal cross section after multiplying branching fractions. The Solid line corresponds to the SM Higgs boson and the broken line corresponds to the bosophilic Higgs boson. . . . . . . . . . . . . . . . . . . . . . . .

6.3 Leading lepton and second leading lepton $p_{T}$ distributions for each dilepton type $(e e, \mu \mu$, and $e \mu)$. Left (Right) plots correspond to the 110 $\mathrm{GeV} / c^{2}\left(160 \mathrm{GeV} / c^{2}\right)$ Higgs boson mass Monte Carlo sample. . . . . . 137

6.4 Upper (Lower) plot shows geometrical and kinematical cut efficiency for each dilepton type for the $W H \rightarrow W W^{*} W^{*} \rightarrow$ dilepton $(W H+Z H \rightarrow$ $V V^{*} V^{*} \rightarrow$ dilepton) signal Monte Carlo samples. Circles, squares, triangles, and diamonds correspond to the dielectron channel, dimuon channel, $e \mu$ channel, and trilepton channel respectively. . . . . . . . . . .

6.5 Comparison between Monte Carlo $Z \rightarrow e e$ and data one. Upper left plot is raw isolation distribution, upper right one is electron $E_{T}$ dependence, lower left one is the efficiency vs cut value, and lower right one is $\eta$ dependence of the efficiency. . . . . . . . . . . . . . . . . . . . . . . . 140

6.6 Comparison between Monte Carlo $Z \rightarrow \mu \mu$ and data one. Upper left plot is raw isolation distribution, upper right one is electron $E_{T}$ dependence, lower left one is the efficiency vs cut value, and lower right one is $\eta$ dependence of the efficiency. . . . . . . . . . . . . . .

6.7 The lepton-isolation features of signal Monte Carlo sample. The Higgs boson mass is $160 \mathrm{GeV} / c^{2}$ and the event type is dielectron. Upper left plot is raw $I S O_{0.4}^{\text {cal }}$ distribution, upper right plot is $E_{T}$ dependence of isolation cut efficiency, lower left plot is the isolation cut efficiency as a function of the cut value, and lower right is the cut efficiency as a function of the Higgs boson mass. . . . . . . . . . . . . . . . . . . . . . 142

6.8 The lepton-isolation features of signal Monte Carlo sample. The Higgs boson mass is $160 \mathrm{GeV} / c^{2}$ and the event type is dimuon. Upper left plot is raw $I S O_{0.4}^{\text {cal }}$ distribution, upper right plot is $E_{T}$ dependence of isolation cut efficiency, lower left plot is the isolation cut efficiency as a function of the cut value, and lower right is the cut efficiency as a function of the Higgs boson mass. . . . . . . . . . . . . . . . . .

6.9 The lepton-isolation features of signal Monte Carlo sample. The Higgs boson mass is $160 \mathrm{GeV} / c^{2}$ and the event type is $e \mu$. Upper left plot is raw $I S O_{0.4}^{\text {cal }}$ distribution, upper right plot is $E_{T}$ dependence of isolation cut efficiency, lower left plot is the isolation cut efficiency as a function of the cut value, and lower right is the cut efficiency as a function of the Higgs boson mass. . . . . . . . . . . . . . . . . . . . . . . . 144

6.10 Upper (Lower) plot is the isolation cut efficiencies as a function of the Higgs boson mass for $W H(W H+Z H)$ production. The circle point is dielectron channel, square one is dimuon channel, triangle one is $e \mu$ channel, and diamond one is trilepton channel. . . . . . . . . . . 
6.11 Upper (Lower) plot shows the isolation cut efficiencies as a function of the Higgs boson mass for the $W H(W H+Z H)$ production. Circles, squares, triangles, and diamonds correspond to the dielectron channel, dimuon channel, $e \mu$ channel, and trilepton channel respectively. . . . . 150

$6.12\left|z_{0}^{l 1}-z_{0}^{l 2}\right|$ distributions of $Z \rightarrow \ell \ell$ events. . . . . . . . . . . . . 151

6.13 Upper (Lower) plot is dilepton selection cuts efficiencies as a function of the Higgs boson mass for $W H(W H+Z H)$ production. The circle point is dielectron channel, square one is dimuon channel, and triangle one is $e \mu$ channel . . . . . . . . . . . . . . . . . . . . . 152

6.14 Upper (Lower) plot is topological selection cuts efficiencies as a function of the Higgs boson mass for $W H(W H+Z H)$ production. The circle point is dielectron channel, square one is dimuon channel, and triangle one is $e \mu$ channel. . . . . . . . . . . . . . . . . . . . . 158

6.15 Level-1 (top) and Level-3 (bottom) tracking efficiencies as a function of $\eta$ measured by CES. . . . . . . . . . . . . . . . . . . . 159

6.16 L2_CEM16 trigger efficiency as a function of $E_{T} \ldots \ldots \ldots \ldots$. . . . . . 159

6.17 Upper (Lower) plot is total signal acceptance as a function of the Higgs boson mass for $W H(W H+Z H)$ production. The circle point is dielectron channel, square one is dimuon channel, triangle one is $e \mu$ channel, and crossed one is the dilepton total (including trilepton events). . . . 160

7.1 Upper limit on the cross section for each mass point from pseudoexperiments. Arrows show the mean and RMS. . . . . . . . . . . 165

7.2 Upper (Lower) plot shows 95\% C.L. upper limits on the production cross section times branching fraction as a function of Higgs boson mass for the $W H(W H+Z H)$ production, together with the cross sections of the Standard Model and of the benchmark scenario for the bosophilic Higgs boson. Results from pseudo-experiments for expected limits are also shown. . . . . . . . . . . . . . . . 166

7.3 CDF Run II preliminary cross section limit for the Standard Model Higgs boson. . . . . . . . . . . . . . . . . . 167 


\section{List of Tables}

1.1 Experimentally measured masses of the fermions [3]. In the Standard Model, neutrinos are defined to be massless. . . . . . . . . . . . .

1.2 Quantum numbers of the fermions in the Standard Model, where $Q$ is the charge, $t$ and $t_{3}$ denote the weak isospin and its third component and $y$ is the weak hypercharge. Members of a given weak isospin multiplet have a common hypercharge. . . . . . . . . . . . . . 4

1.3 Quantum numbers of the bosons in the Standard Model, where $q$ is the charge, $t$ and $t_{3}$ denote the weak isospin and its third component. . . . 5

2.1 Design parameters of the Central Outer Tracker at CDF. . . . . . . . . 35

2.2 Summary of the CDF calorimeters. The different components CEM, CHA, etc. are described in the text. . . . . . . . . . . . . . . 38

2.3 Design parameters of the CDF II Muon Detectors. Pion interaction lengths and multiple scattering are computed at a reference angle of $\theta=55$ degree in CMX, and show a range of values for the BMU. . . . . 40

3.1 Muon reconstruction criteria. . . . . . . . . . . . . . . . . 64

3.2 Criteria for central tight electron and muon candidates . . . . . . . . . 72

3.3 Primary vertex and lepton selection cuts. . . . . . . . . . . . . 78

3.4 Dilepton selection cuts. . . . . . . . . . . . . . . . . . 78

3.5 Physics objects used to identify and remove $Z$ bosons. . . . . . . . . . 79

3.6 Number of events passing like-sign dilepton selection. . . . . . . . . . . 79

4.1 Summary of the Monte Carlo samples; number of generated events, accepted cross section $\sigma \cdot B r(\mathrm{pb})$, and comments. . . . . . . . . . . . 82

4.2 Partner track finding efficiencies for five $E_{T}$ regions of the conversion electrons. . . . . . . . . . . . . . . . . 102

4.3 Conversion tagging cut efficiencies for four $E_{T}$ cuts of the conversion electrons. . . . . . . . . . . . . . . . . . 107

4.4 Partner track finding efficiencies above $2 \mathrm{GeV} / c$ for four $E_{T}$ cuts of the conversion electrons. . . . . . . . . . . . . . . . . . 108 
4.5 Overall conversion tagging efficiencies for five $E_{T}$ regions of the conversion electrons. . . . . . . . . . . . . . . . . . . . . . 110

4.6 Residual photon conversion ratios for five $E_{T}$ regions of the conversion

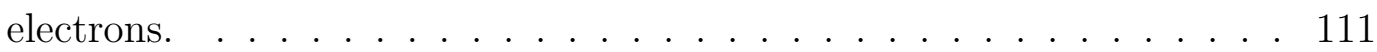

5.1 Topological cuts . . . . . . . . . . . . . . . . 126

5.2 Breakdown of background components and observed events for each region. . . . . . . . . . . . . . . . . . . 127

$6.1 z_{\mathrm{vtx}}$ cut efficiencies and scale factors for the different Monte Carlo samples. We refereed $0.951 \pm 0.006$ as data-derived $z_{\mathrm{vtx}}$ cut efficiency [78]. Combined value is the average of five different Monte Carlo sample. . . 134

6.2 Isolation cut efficiencies for Monte Carlo $Z \rightarrow \ell \ell$ and data for each lepton type. . . . . . . . . . . . . . . . . . 139

6.3 Electron identification cut efficiencies for data $Z \rightarrow e e$ and the Monte Carlo samples. The scale factors (Data/Monte Carlo) are listed in the rightmost column. . . . . . . . . . . . . . . . . . 147

6.4 Muon identification cut efficiencies in $Z \rightarrow \mu \mu$ for data and the Monte Carlo samples for each dimuon combination (CMUP-CMUP, CMX-CMUP, CMX-CMX, and CMUP-CMX). The scale factors (Data/Monte Carlo) are listed in the rightmost column. The combined value is the weighted average value. . . . . . . . . . . . . . . . . . . . . . . . . . . 148

$6.5 z_{0}^{l 1}-z_{0}^{l 2}$ cut efficiencies for data $Z \rightarrow \ell \ell$ and Monte Carlo for each lepton type. The scale factor (Data/Monte Carlo) are listed in right row. . . . 149

6.6 The requirements for the high $p_{T}$ electron trigger and the backup triggers. 153

6.7 Total signal acceptance as a function of the Higgs boson mass. The denominator of the acceptance is $W H(W H+Z H) \rightarrow W W W(V V V) \rightarrow$ dilepton or trilepton events. . . . . . . . . . . . . 156

6.8 Systematic error sources and errors. . . . . . . . . . . . . . . . 157

7.1 Expected limit and observed limit for each Higgs boson mass point. The error is taken from the RMS of limit distribution. . . . . . . . . . . . . 164 


\section{Chapter 1}

\section{Introduction}

Elementary particle physics is the study of the fundamental particles of matter and their interactions. By "elementary" physicists mean that these particles have no measurable internal structure or components. They can thus be treated as ideal or point-like particles to the smallest scale of size available to physicists in the modern era, $10^{-19}$ meters. By "fundamental" we mean that the physical properties such as mass, electric charge, and spin of these infinitesimal particles underlies at the physical world of our everyday experience. These physical properties dictate how the fundamental particles themselves compose more complex particles and atoms. The properties of the fundamental particles then govern how those atoms in turn build molecules, cells, organisms and all bulk matter.

The laws of classical biology, chemistry and physics all rely on bulk properties of materials and classical mechanics, i.e. many millions of elementary particles moving slowly compared to the speed of light $\left(c=2.9979 \times 10^{8}\right.$ meters/second $)$. When dealing with individual particles or atoms physicists must replace the laws of classical mechanics with those of quantum mechanics. When dealing with particles moving very quickly, generally greater than $\frac{1}{10} c$, physicists must replace classical mechanics with relativistic mechanics. In the case of both extremes - the regime of modern high-energy particles physics - relativistic mechanics and quantum mechanics must be combined into a relativistic quantum field theory (QFT).

The fundamental particles currently known to physicists are the quarks, leptons, and gauge bosons. The gauge bosons (integer spin) mediate the interactions between the quarks $(q)$ and leptons $(\ell)$, which have $\frac{1}{2}$ - integer spin. One or more gauge bosons have been observed for each of the fundamental forces currently described by physicists with a working QFT: electromagnetic, weak nuclear, and strong nuclear. The QFT describing the electromagnetic and nuclear interactions between quarks, leptons, and gauge bosons is called the Standard Model (SM) of particle physics. 


\section{$1.1 \quad$ Standard Model}

The Standard Model has his roots in gauge theory and exploits some of the symmetries that are inherent in many physical theories. The following sections describe some of the different parts of the Standard Model. For a more comprehensive description of it, see for instance [1].

\subsubsection{Standard Model Elements}

The matter fields that constitute the Standard Model can be classified into two group according to their spins. These are the fermions which are spin $\frac{1}{2}$ particles and the bosons which are spin-0 or spin-1. Theory are described in the following sections.

\section{The Fermions}

These particles are characterized by their spin- $\frac{1}{2}$ nature and are thus governed by FermiDirac statistics. They can be split into two groups, quarks and leptons, which are distinguished by the charges associated with them. There are the six quarks ( $\mathrm{u}, \mathrm{d}, \mathrm{c}, \mathrm{s}$, $\mathrm{t}$, and $\mathrm{b}$ ) and six leptons ( electron, muon, and tau with their corresponding neutrinos). Quarks carry the color charge of the strong interactions of Quantum Chromodynamics (QCD) and fractional electric charge, whereas leptons are colorless and have integer electric charge. In addition, the fermions can be split into generations which correspond to doublets of left-handed and singlets of right-handed quarks and leptons of increasing mass. This separation is based on the empirical evidence of the chirality of the weak interactions and corresponds to massless neutrinos. Table 1.1 and 1.2 show the fermions and their properties.

\section{The Bosons}

There are four known forces which act on matter. Three have a basis within the SM , electromagnetic and weak, combined in the electroweak interaction, and strong. The gravitational force is negligibly small at the energy scales at which the SM is thought to be relevant, and it is not included. The electromagnetic, weak, and strong forces are mediated by the spin-1 gauge bosons, which are shown in Table 1.3 along with their masses. Although the gravitational interaction is not featured in the SM, it is thought to be mediated by a spin- 2 gauge boson, known as the graviton. For a possible realization of Quantum Gravity and the graviton, which constitutes physics beyond the Standard Model, see for instance [2]. 


\begin{tabular}{cccc}
\hline \hline Leptons & Mass & Quarks & Mass \\
\hline$\nu_{e}$ & $<3 \mathrm{eV} / c^{2}$ & $u$ & $1.5-4.5 \mathrm{MeV} / c^{2}$ \\
$e$ & $0.511 \mathrm{MeV} / c^{2}$ & $d$ & $5.0-8.5 \mathrm{MeV} / c^{2}$ \\
$\nu_{\mu}$ & $<0.19 \mathrm{MeV} / c^{2}$ & $c$ & $1.0-1.4 \mathrm{GeV} / c^{2}$ \\
$\mu$ & $105.66 \mathrm{MeV} / c^{2}$ & $s$ & $80-155 \mathrm{MeV} / c^{2}$ \\
$\nu_{\tau}$ & $<18.2 \mathrm{MeV} / c^{2}$ & $t$ & $174.3 \mathrm{GeV} / c^{2}$ \\
$\tau$ & $1.777 \mathrm{GeV} / c^{2}$ & $b$ & $4.0-4.5 \mathrm{GeV} / c^{2}$ \\
\hline \hline
\end{tabular}

Table 1.1: Experimentally measured masses of the fermions [3]. In the Standard Model, neutrinos are defined to be massless.

\subsubsection{Standard Model Structure}

The Standard Model is based on the local gauge symmetry of the fundamental $\mathrm{SU}(2)_{L} \otimes \mathrm{U}(1)_{Y}$ electroweak and the $\mathrm{SU}(3)_{C}$ strong interactions in particle physics:

$$
\mathrm{SU}(3)_{C} \bigotimes \mathrm{SU}(2)_{L} \bigotimes \mathrm{U}(1)_{Y}
$$

Here $C$ stands for the color charge carried by the strong interactions, $L$ denotes the fact that the left-handed components of the fermions form doublets, and $Y$ represents the weak hypercharge carried by the electroweak interactions, defined as:

$$
Y=2\left(\frac{Q}{e}-t_{3}\right)
$$

where $Q$ is the charge, $t$ and $t_{3}$ denote the weak isospin and its third component.

By demanding local gauge invariance of the Standard Model Lagrangian, massless spin-1 fields, mediating the interactions, are required. However, this makes the theory inconsistent with experimental observation of the massive electroweak bosons $W^{ \pm}$and $Z^{0}$. The mechanism that generates masses in the SM is based on spontaneous symmetry breaking of the electroweak $\mathrm{SU}(2)_{L} \otimes \mathrm{UU}(1)_{Y}$ symmetry. This causes the decoupling of the weak and electromagnetic forces while preserving the local gauge invariance of the whole theory. A consequence of spontaneous symmetry breaking is the prediction of there being a massive scalar (spin-0) particle known as the Higgs boson (Table 1.3), as yet undiscovered experimentally. The following section explain the above in more detail. The electromagnetic interaction, based on the symmetry group $\mathrm{U}(1)_{Q}$, is used as an example of how local gauge invariance requires the existence of an extra massless vector field, in this case identified with the photon. 


\begin{tabular}{|c|c|c|c|c|c|c|}
\hline \multicolumn{3}{|c|}{ Generation } & \multicolumn{4}{|c|}{ Quantum number } \\
\hline $1 \mathrm{st}$ & 2nd & $3 r d$ & $Q$ & $t$ & $t_{3}$ & $y$ \\
\hline \multicolumn{7}{|c|}{$\underline{\text { Leptons }}$} \\
\hline$\nu_{e}$ & $\nu_{\mu}$ & $\nu_{\tau}$ & 0 & $1 / 2$ & $+1 / 2$ & -1 \\
\hline$e$ & $\mu$ & $\tau$ & -1 & $1 / 2$ & $-1 / 2$ & -1 \\
\hline$e_{R}$ & $\mu_{R}$ & $\tau_{R}$ & -1 & 0 & 0 & -2 \\
\hline \multicolumn{7}{|c|}{ Quarks } \\
\hline$u$ & $c)$ & $t)$ & $+2 / 3$ & $1 / 2$ & $+1 / 2$ & $1 / 3$ \\
\hline$d)$ & $s)$ & $b)$ & $-1 / 3$ & $1 / 2$ & $-1 / 2$ & $1 / 3$ \\
\hline$u_{R}$ & $c_{R}$ & $t_{R}$ & $+2 / 3$ & 0 & 0 & $+4 / 3$ \\
\hline$d_{R}$ & $s_{R}$ & $b_{R}$ & $-1 / 3$ & 0 & 0 & $-2 / 3$ \\
\hline
\end{tabular}

Table 1.2: Quantum numbers of the fermions in the Standard Model, where $Q$ is the charge, $t$ and $t_{3}$ denote the weak isospin and its third component and $y$ is the weak hypercharge. Members of a given weak isospin multiplet have a common hypercharge.

\subsubsection{Quantum Electrodynamics: $\mathrm{U}(1)_{Q}$}

Quantum Electrodynamics (QED) is the gauge theory of electromagnetic interaction. For a free Dirac field $\psi$ with spin $s=\frac{1}{2}$, mass $m$ and electric charge $e Q$, the corresponding equation of motion and the Lagrangian are:

$$
\begin{gathered}
\left(i \gamma^{\mu} \partial_{\mu}-m\right) \psi=0, \\
L_{\text {free }}=\bar{\psi}\left(i \gamma^{\mu} \partial_{\mu}-m\right) \psi .
\end{gathered}
$$

The Lagrangian can be seen to be invariant under the global U(1) transformations, $\psi \rightarrow e^{i Q \theta} \psi, \bar{\psi} \rightarrow \bar{\psi} e^{-i Q \theta}$, and $\partial_{\mu} \psi \rightarrow e^{i Q \theta} \partial_{\mu} \psi$, where $Q \theta$ is a global phase and $\theta$ is a continuous parameter. Noether's Theorem states that if a system is invariant under a continuous transformation, then there is a conserved current and associated charge. Therefore, the global $\mathrm{U}(1)$ invariance of $L_{\text {free }}$ implies the conservation of electromagnetic charge, $e Q$, and current, $J_{\mu}$,

$$
J_{\mu}=\bar{\psi} \gamma_{\mu} e Q \psi, \quad \partial_{\mu} J^{\mu}=0, \quad e Q=\int d^{3} x J^{0}(x) .
$$

If we make the global transformation into a local one, i.e., the continuous parameter $\theta$ is allowed to depend on the space-time point $x$, then the Lagrangian is now only invariant if a vector field $A_{\mu}$ is introduced, which transforms under the local gauge transformations as $A_{\mu} \rightarrow \frac{1}{e} \partial_{\mu} \theta(x)$. $A_{\mu}$ is identified with the mediator of the electromagnetic force, the photon. 


\begin{tabular}{|c|c|c|c|c|c|}
\hline Boson & Interaction & Mass & $q$ & $t$ & $t_{3}$ \\
\hline \multicolumn{6}{|c|}{ Vector Bosons } \\
\hline$W^{+}$ & weak & $80.42 \mathrm{GeV} / c^{2}$ & +1 & +1 & +1 \\
\hline$W^{-}$ & weak & $80.42 \mathrm{GeV} / c^{2}$ & -1 & +1 & -1 \\
\hline$Z^{0}$ & electroweak & $91.18 \mathrm{GeV} / c^{2}$ & 0 & +1 & 0 \\
\hline$\gamma$ & QED & 0 & 0 & 0 & 0 \\
\hline gluon & $\mathrm{QCD}$ & 0 & 0 & 0 & 0 \\
\hline \multicolumn{6}{|c|}{ Scalar Boson } \\
\hline Higgs & Yukawa & $>114.4 \mathrm{GeV} / c^{2}$ & 0 & 0 & 0 \\
\hline
\end{tabular}

Table 1.3: Quantum numbers of the bosons in the Standard Model, where $q$ is the charge, $t$ and $t_{3}$ denote the weak isospin and its third component.

In order to complete the expression for the Lagrangian a term has to be introduced to account for the propagation of the vector field, which must also be gauge invariant. The so called kinetic term is given in terms of the field strength tensor, $F_{\mu \nu}=\partial_{\mu} A_{\nu}-$ $\partial_{\nu} A_{\mu}$. Thus, the Lagrangian of the Quantum Electrodynamics is

$$
L_{Q E D}=\bar{\psi}\left(i \gamma^{\mu}\left(\partial_{\mu}+i e Q A_{\mu}\right)-m\right) \psi-\frac{1}{4} F_{\mu \nu} F^{\mu \nu} .
$$

The addition of a mass term $\frac{1}{2} m^{2} A_{\mu} A^{\mu}$ would cause the QED Lagrangian to change under a local gauge transformation and is therefore not allowed. Thus the gauge field, the photon, is massless, which is consistent with experimental observation and ensures that the electromagnetic force has infinite range.

\subsubsection{Quantum Chromodynamics: $\mathrm{SU}(3)_{C}$}

Quantum Chromodynamics is the gauge theory for strong interactions. It is based on the symmetry group $\mathrm{SU}(3)_{C}$ of phase transformations on the quark color fields. The quark color charge, conventionally taken to be red, blue, and green, was originally postulated to preserve the Pauli exclusion principle for states such as $\Omega^{-}$and $\Delta^{++}$ which would otherwise be described by symmetric wave-functions. The color quantum number has since been shown to accurately describe many other aspects of the strong interaction. Local gauge invariance of the non-Abelian $\mathrm{SU}(3)_{C}$ results in the eight massless vector fields, the gluons, which themselves carry the color charge. The QCD Lagrangian is written in terms of the quark fields $\psi=(r, g, b)$ and contains in addition the kinetic term for the gluon fields,

$$
L_{Q C D}=\bar{\psi}\left(i \gamma^{\mu} D_{\mu}-m\right) \psi-\frac{1}{4} F_{\mu \nu}^{a} F^{a \mu \nu}
$$




$$
D_{\mu}=\partial_{\mu}+i g_{s} A_{\mu}^{a} \frac{\lambda^{a}}{2}=\partial_{\mu}+i g_{s} \sum_{a=1}^{8} A_{\mu}^{a} \frac{\lambda^{a}}{2}
$$

The new element with respect to the QED Lagrangian defined in equation 1.5 is the set of eight $\mathrm{SU}(3) 3 \times 3$ matrices $\lambda_{a}$, numbered by the gluon index $a=1, \cdots, 8$. They fulfill the $\mathrm{SU}(3)$ commutation relations: $\left[\lambda_{a} / 2, \lambda_{b} / 2\right]=i C_{a b c} \lambda_{c} / 2$, where $C_{a b c}$ are the $\mathrm{SU}(3)$ algebra structure constants. The gluon field tensors $F_{\mu \nu}^{a}$ are defined as

$$
F_{\mu \nu}^{a}=\partial_{\mu} A_{\nu}^{a}-\partial_{\nu} A_{\mu}^{a}+g_{s} C_{a b c} A_{\mu}^{b} A_{\nu}^{c}
$$

The last term in Equation 1.8 is responsible for gluon self-interactions, which accounts for both the asymptotic freedom of quarks and their confinement within color singlet states. Gluon anti-screening of the quark color charge causes the strong coupling constant $\alpha_{S}$ to decrease with decreasing distance, such that quarks behave as free particles at high momentum transfers. As a $q \bar{q}$ pair is separated $\alpha_{S}$ increases. Field lines stretch into a tube until the field gains sufficient energy to create a new $q \bar{q}$ pair; no finite amount of energy can liberate a single quark. This behavior is in contrast to the running coupling constant of QED, the fine-structure constant $\alpha$, which increases with increasing $q^{2}$ (the transferred momentum) due to the lack of self-interactions between photons.

By means of Deep Inelastic Scattering (DIS) experiments, it has been shown that the point-like quarks are the constituents of the nucleons, proton and neutron. Indeed it is possible to reconstruct and explain the properties of the nucleons from the quantum numbers of these constituents. For this purpose, two different types of quarks are needed, $\mathrm{u}$ and $\mathrm{d}$. As the quarks and the nucleons both have spin $\frac{1}{2}$, the nucleons have to be made of at least three quarks: the proton has two u-quarks and one d-quark, while the neutron has two d-quarks and one u-quark. These three quarks determine the quantum numbers of the nucleons and are called "valence quarks". A "sea" of virtual quark-antiquark pairs, formed by u-d as well as the other quark flavors, is also present in the nucleon, but the contribution from charm and more massive quarks is heavily suppressed. Their effective quantum numbers average to zero such that they don't alter the quantum numbers of the nucleon. They are also visible in DIS interactions because of their electric charge, but they carry a smaller fraction of the nucleon momentum. Quarks inside the nucleon produce gluons by the process $q \rightarrow q+g$. The sea quarks are produced by gluon splitting into $q \bar{q}$ pairs through the process $g \rightarrow q \bar{q}$. The fractional momentum of the nucleon carried by the valence quarks, sea quarks and gluons changes as the momentum transfer squared $\left(q^{2}\right)$ of the probe is increased. As $q^{2}$ increases the fractional momenta carried by the sea quarks and gluons increase at the expense of the valence quarks. The distribution of the quarks and gluons in the nucleons is described by functions called Structure Functions or Parton Distribution Functions (PDFs). For a more complete treatment of this subject, see [4]. 
The lepton doublets participating in the weak interaction are the doublets listed in Table 1.2. In the quark sector, the weak interaction quark doublets are a linear combination of the strong interaction mass eigenstates. This idea was first postulated by Cabibbo for $\mathrm{u}, \mathrm{d}$, and s quarks to accommodate both quark-lepton universality and the different decay rates of fully leptonic muon decay and strangeness changing and conserving hadronic decays. The principle has been extended to a second generation including charm using the GIM (Glashow, Iliopoulos and Maiani) mechanism in order to explain the absence of flavor-changing neutral currents and to a third generation incorporating $\mathrm{t}$ and $\mathrm{b}$ quarks to account for small $\mathrm{CP}$-violating effects (where $\mathrm{C}$ refers to charge conjugation and $\mathrm{P}$ to parity). The $3 \times 3$ Cabibbo-Kobayashi-Maskawa (CKM) matrix incorporates the mixing terms of this mechanism. It is expressed as

$$
V_{C K M}=\left[\begin{array}{lll}
V_{u d} & V_{u s} & V_{u b} \\
V_{c d} & V_{c s} & V_{c b} \\
V_{t d} & V_{t s} & V_{t b}
\end{array}\right] .
$$

Being unitary, it can be parametrized in terms of three angles and a single phase. Offdiagonal terms describe flavor-changing charged currents. $V_{u d}$ is the most accurately measured, whereas $V_{c b}$ and $V_{u b}$ are small but yet non-zero and the most difficult to measure. The most accurate measurement of $\left|V_{c s}\right|$ to data has been made by LEP [3].

\subsubsection{Electroweak Theory: $\mathrm{SU}(2)_{L} \otimes \mathrm{U}(1)_{Y}$}

The SM electroweak theory is based upon the symmetry group $\mathrm{SU}(2)_{L} \otimes \mathrm{U}(1)_{Y}$, which is a local symmetry of the electroweak Lagrangian. $\mathrm{SU}(2)_{L}$ is the isospin group which acts only on the left-handed fermions and $\mathrm{U}(1)_{Y}$ is the weak hypercharge group. Within the electroweak formalism the electromagnetic and weak interactions are unified, and the $\mathrm{U}(1)_{Q}$ symmetry group which was described before appears as a subgroup of the total electroweak group. The local gauge transformations of the electroweak theory are obtained by combining the local transformations for the $\mathrm{U}(1)$ and $\mathrm{SU}(2)$ groups, thus:

$$
U=\exp \left(-i\left(\frac{g}{2} \sigma_{i} \theta_{i}(x)+\frac{g^{\prime}}{2} Y \theta^{\prime}(x)\right)\right.
$$

under which the doublet

$$
\psi_{L}=\frac{1-\gamma_{5}}{2}\left(\begin{array}{c}
\psi_{\nu_{\ell}} \\
\psi_{\ell}
\end{array}\right)
$$

and the singlet

$$
\psi_{L}=\frac{1+\gamma_{5}}{2} \psi_{\ell}
$$

transform, $\psi_{L}^{\prime}=U \psi_{L}$ and $\psi_{R}^{\prime}=U \psi_{R}$. here $\sigma_{i}$ are the Pauli spin matrices and $Y$ is the diagonal matrix of the weak hypercharges. Performing these local transformations 
and demanding gauge invariance generates interactions between the particles in the form of four vector fields. These are three fields identified with the $\mathrm{SU}(2)_{L}$ transformations, $\left(W_{\mu}^{1}, W_{\mu}^{2}, W_{\mu}^{3}\right)$ and one field belonging to the $\mathrm{U}(1)_{Y}$ transformations, $B_{\mu}$. The electroweak Lagrangian is as follows:

$$
\begin{aligned}
L_{E W}= & \bar{\psi} i \gamma^{\mu} \partial_{\mu} \psi \\
& -\bar{\psi}_{L} \gamma^{\mu}\left(\frac{g}{2} \boldsymbol{\sigma} \cdot \boldsymbol{W}_{\mu}+\frac{g^{\prime}}{2} Y_{L} B_{\mu}\right) \psi_{L} \\
& -\bar{\psi}_{R} \gamma^{\mu} \frac{g^{\prime}}{2} Y_{R} B_{\mu} \psi_{R} \\
& -\frac{1}{4} \boldsymbol{W}_{\mu \nu} \boldsymbol{W}^{\mu \nu}-\frac{1}{4} B_{\mu \nu} B^{\mu \nu}
\end{aligned}
$$

The U(1) gauge field, $B_{\mu}$, couples to weak hypercharge with coupling $\frac{g^{\prime}}{2}$. The triplet of gauge fields $W_{\mu}^{a}$ couples to weak isospin with coupling $g$. As in the case of QED, gauge invariance is preserved by ensuring that the fields are massless. The massless fields in the above Lagrangian can be combined to form the physical fields of the electroweak theory. The charged $W^{ \pm}$bosons are formed from the $W_{\mu}^{1}$ and $W_{\mu}^{2}$ and couple to lefthanded chirality states. The $Z^{0}$ and photon couple to both left- and right-handed fermions and are formed as orthogonal linear combinations of the $W_{\mu}^{3}$ and $B_{\mu}$ fields.

$$
\begin{aligned}
W_{\mu}^{ \pm} & =\frac{1}{\sqrt{2}}\left(W_{\mu}^{1} \mp i W_{\mu}^{2}\right), \\
Z_{\mu} & =\cos \theta_{W} W_{\mu}^{3}-\sin \theta_{W} B_{\mu}, \\
A_{\mu} & =\sin \theta_{W} W_{\mu}^{3}+\cos \theta_{W} B_{\mu},
\end{aligned}
$$

where $\theta_{W}$ is the Weinberg or weak mixing angle. It relates the couplings of the electromagnetic and weak interactions according to $g \sin \theta_{W}=g^{\prime} \cos \theta_{W}=e$. As with QED the addition of a mass term of the form $\frac{1}{2} m^{2} V_{\mu} V^{\mu}$ for the bosonic field would break gauge invariance.

\subsubsection{Electroweak Symmetry Breaking}

Electroweak theory predicts the existence of four gauge bosons, $\gamma, W^{ \pm}$, and $Z^{0}$. These are necessarily massless in order to preserve the local gauge invariance of the theory. However, it is known that $W^{ \pm}$and $Z^{0}$ bosons of the weak interaction have mass. The Higgs mechanism [5], [6], and [7] provides a possible explanation of the origin of the masses through gauge invariant spontaneous symmetry breaking of the electroweak sector. It is an extension of the Goldstone Theorem which states that if a Lagrangian has a global symmetry which is not a symmetry of the vacuum (i.e.the ground state) then there must exist one massless boson, scalar or pseudo-scalar, associated to each generator which does not annihilate the vacuum. These modes are known 


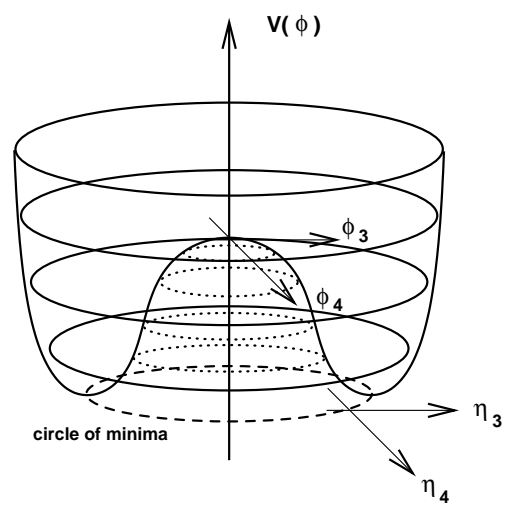

Figure 1.1: The Higgs potential $V$ for a complex scalar field with $\mu^{2}<0$ and $\lambda>0$.

as the Goldstone Bosons. In the Higgs mechanism a weak isospin doublet of complex scalar fields $\phi^{0}(x)$ and $\phi^{+}(x)$ is introduced which must belong to the $\mathrm{SU}(2)_{L} \otimes \mathrm{U}(1)_{Y}$ multiplets and along with the scalar potential $V(\phi)$

$$
\begin{gathered}
\phi(x)=\left(\begin{array}{c}
\phi^{+}(x) \\
\phi^{0}(x)
\end{array}\right)=\frac{1}{\sqrt{2}}\left(\begin{array}{c}
\phi_{1}(x)+i \phi_{2}(x) \\
\phi_{3}(x)+i \phi_{4}(x)
\end{array}\right) \\
V(\phi)=\mu^{2} \phi^{\dagger} \phi+\lambda\left(\phi^{\dagger} \phi\right)^{2}, \quad \lambda>0 .
\end{gathered}
$$

This gives a contribution to the electroweak Lagrangian, $L_{H i g g s}$, where $D_{\mu}$ is the covariant derivative:

$$
L_{\text {Higgs }}=\left(D_{\mu} \phi\right)^{\dagger}\left(D^{\mu} \phi\right)-V(\phi), \quad D^{\mu}=\partial^{\mu}-i \frac{g}{2} \boldsymbol{\sigma} \cdot \boldsymbol{W}^{\mu}-i \frac{g^{\prime}}{2} Y B^{\mu} .
$$

The minimum of $V$ corresponds to the ground state of the system or vacuum which is at $|\phi|=0$ for $\mu^{2}>0$, but for the choice $\mu^{2}<0$ the minimum shifts to

$$
\left|\phi^{2}\right|=\phi^{\dagger} \phi=\frac{1}{2}\left(\phi_{1}^{2}+\phi_{2}^{2}+\phi_{3}^{2}+\phi_{4}^{2}\right)=-\frac{\mu^{2}}{2 \lambda}=\frac{v^{2}}{2},
$$

where $v$ is the vacuum expectation value. The above solutions are now degenerate and any point satisfying the equation of a circle is a ground state. The perturbation procedure of Feynman calculus starts with fields which are fluctuations from the vacuum ground state. This leads to the definition of two new field variables $\eta_{1}$ and $\eta_{2}$, so that the potential is of the form in Figure 1.1. They have their origin at an arbitrarily chosen minimum

$$
\begin{aligned}
|\langle 0|\phi| 0\rangle| & =\frac{1}{\sqrt{2}}\left(\begin{array}{c}
0 \\
v
\end{array}\right), \quad \text { where } \phi_{1}=\phi_{2}=\phi_{4}=0, \quad \phi_{3}^{2}=v^{2} \\
\eta_{3} & =\phi_{3}-v \text { and } \eta_{4}=\phi_{4} .
\end{aligned}
$$


The symmetry of the Lagrangian becomes hidden by the choice of a particular minimum. The Lagrangian expressed in terms of the new fields reveals a massive scalar particle $\eta_{3}$ of mass $M_{H i g g s}=\sqrt{2 \lambda v^{2}}$, the Higgs boson $H$, and three massless Goldstone bosons $\phi_{1}, \phi_{2}$, and $\eta_{4}$. These unwanted Goldstone bosons can be removed by applying a unitary gauge transformation to $\phi(x)$ such that only the real Higgs field remains,

$$
\phi(x)=U \phi(x)=\frac{1}{\sqrt{2}}\left(\begin{array}{c}
0 \\
v+H(x)
\end{array}\right) .
$$

\section{Electroweak Boson Masses}

In doing so, the three Goldstone bosons disappear from the theory: their corresponding degrees of freedom are eaten by the $W^{ \pm}$and $Z^{0}$ fields which acquire mass and a third, longitudinal, polarization state. By invoking the Higgs mechanism, expanding the fields about the chosen vacuum $\phi_{0}$ and gauging away the Goldstone bosons, the gauge boson masses are generated:

$$
M_{W}=\frac{g v}{2}, \quad M_{Z}=\frac{g v}{2 \cos \theta_{W}}, \quad M_{\gamma}=0 .
$$

\section{Fermions Masses}

As well as coupling to the gauge fields $\mathrm{W}_{\mu}$ and $B_{\mu}$, the Higgs field couples to the fermion matter fields to generate their masses. The coupling of the Higgs field to a fermion pair is parametrized by an arbitrary Yukawa coupling constant $\lambda_{f}=m_{f} \sqrt{2} / v$, different for each fermion and proportional to its mass $m_{f}$. Lepton number conservation is assumed within the SM, giving a diagonal lepton mass matrix. The lack of quark generation number conservation in electroweak interactions means that the observed physical mass eigenstates of quarks are not eigenstates of weak isospin. The level of quark mixing is parametrized in terms of the Cabibbo-Kobayashi-Maskawa mixing matrix.

\subsubsection{Renormalizability}

Calculations within the SM have to be performed using perturbative expansions in terms of the strengths of the couplings. This is because loops of particles can be added to the process without altering the final state. These series are infinite and as such the individual terms of the loop can diverge making calculations impossible. The calculations can be make non-divergent in a theory which is renormalizable. The process of renormalization relates the physical masses and charges of the calculable theory to experimentally unobservable "bare" masses and charges which absorb the divergences. It was shown by t'Hooft and Veltman [8], [9], and [10] that gauge theory, such as the SM, is renormalizable. 


\subsubsection{Input to the Standard Model}

The Standard Model theory was developed over a period of years from both the theoretical and empirical discoveries. The theory has predictive power, yet it still requires 18 empirically determined parameters. These are:

- $\alpha_{S}$ the strength of the coupling of strong interaction,

- $g$ and $g^{\prime}$, the strength of the electroweak couplings,

- $M_{\text {Higgs }}$ the mass of the Higgs boson and $v$ the vacuum expectation value of the Higgs potential,

- The Yukawa couplings, $\lambda_{f}$, of the nine massive fermions,

- The four parameters of the CKM matrix describing the quark mixing.

This large number of free parameters and their arbitrary values are one reason why it is thought that the Standard Model is not a final theory.

\subsection{An Energy Scale $\Lambda$ when the Standard Model breaks down}

As described above, the Standard Model is clearly a very good approximation to the physics of elementary particles and their interactions at an energy scale of $O\left(10^{2} \mathrm{GeV}\right)$ and below. However, theoretical considerations teach us that the Standard Model is not the ultimate theory of the fundamental particles and their interactions. At an energy scale above the Plank scale, $M_{P L} \sim 10^{19} \mathrm{GeV}$, quantum gravitational effects become significant and the Standard Model must be replaced by a more fundamental theory that incorporates gravity [2]. It is also possible that the Standard Model breaks down at some energy scale (called $\Lambda$ ) below the Plank scale. In this case, the Standard Model degrees of freedom are no longer adequate for describing the theory above $\Lambda$ and new physics must become relevant. One possible signal of this occurrence lies in the behavior of the Standard Model couplings. The Standard Model is not an asymptotically free theory since some of the couplings (e.g., the $\mathrm{U}(1)$ gauge coupling, the Higgs-top-quark Yukawa coupling, and the Higgs self-coupling) eventually blow up at some high energy scale. Among these couplings, only the Higgs self-coupling may blow up at an energy scale below $M_{P L}$. Of course, there may be other experimental or theoretical hints that new degrees of freedom exit at some high energy scale below $M_{P L}$.

It is clear from the above discussion that the Standard Model is not a fundamental theory; at best, it is an effective field theory. At an energy scale below $\Lambda$ the Standard 
Model provides an extremely good description of all observable phenomena. Therefore, an essential question that future experiments must address is: what is the minimum scale $\Lambda$ at which new physics beyond the Standard Model must enter? The search for the the origin of electroweak symmetry breaking and the quest to identify $\Lambda$ are intimately tied together. We can consider two scenarios [11]. In the first scenario, electroweak symmetry breaking dynamics results in the existence of a single Higgs boson as posited by the Standard Model. In this case, one would ask whether new phenomena beyond the Standard Model must enter at an energy scale $\Lambda$ that is accessible to experiment. In the second scenario, electroweak symmetry breaking dynamics dose not result in a weakly-coupled Higgs boson as assumed in the Standard Model. In this case, the effective theory that describes current data is a theory that contains the Standard Model fields excluding the Higgs boson. In such an approach, the latter effective field theory must break down at $\Lambda \sim O(1 \mathrm{TeV})$ in order to restore the unitarity of the theory, and new physics associated with the electroweak symmetry breaking dynamics must enter.

\subsection{Present Status of the SM Higgs Boson Search}

Although current data provides no direct evidence to distinguish between the above two scenarios, there is indirect evidence that could be interpreted as favoring the first approach. Namely, the global Standard Model fit to electroweak data takes the Higgs boson mass as a variable to be fitted. The results of the LEP Electroweak Working Group analysis yields [12]:

$$
M_{\text {Higgs }}=81_{-33}^{+52} \mathrm{GeV} / c^{2} .
$$

In fact direct searches at LEP show no evidence for the Higgs boson, and imply the $M_{\text {Higgs }}>114.4 \mathrm{GeV} / c^{2}$ at the $95 \%$ C.L. [13]. And it probably is more useful to quote the $95 \%$ C.L. upper limit that is obtained in the global Standard Model fit [13]:

$$
M_{\text {Higgs }}<193 \mathrm{GeV} / c^{2} \text { at } 95 \% \text { C.L.. }
$$

These results reflect the logarithmic sensitivity to the Higgs boson mass via the virtual Higgs boson loop contributions to the various electroweak observables. The Higgs boson mass range above is consistent with a weakly-coupled Higgs scalar boson that is expected to emerge from the Standard Model scalar dynamics.

Henceforth, we shall take the above result as an indication that the Standard Model (with a weakly-coupled Higgs boson as suggested above) is the appropriate effective field theory at the $100 \mathrm{GeV}$ scale. If this is the case, then the eventual discovery of the Higgs boson will have a profound effect on the determination of $\Lambda$, the scale at which the Standard Model must break down. The key parameter for constraining $\Lambda$ is the 


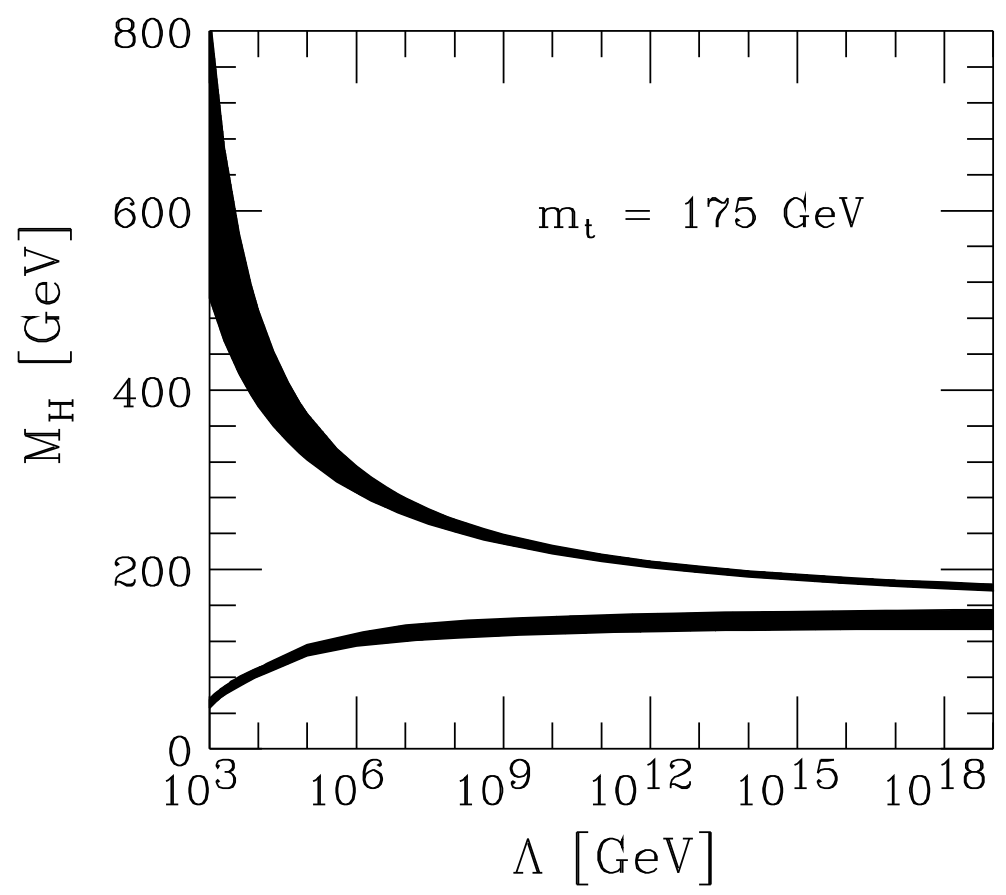

Figure 1.2: The lower [15] and the upper [14] Higgs boson mass bounds as a function of the energy scale $\lambda$ at which the Standard Model breaks down, assuming $M_{t o p}=$ $175 \mathrm{GeV} / c^{2}$ and $\alpha_{s}\left(M_{Z}\right)=0.118$. The shaded areas above reflect the theoretical uncertainties in the calculations of the Higgs boson mass bounds. This figure is taken from [16].

Higgs boson mass, $M_{\text {Higgs }}$. If $M_{\text {Higgs }}$ is too large, then the Higgs boson self-coupling blows up at some scale $\Lambda$ below the Plank scale [14]. If $M_{\text {Higgs }}$ is too small, then the Higgs potential develops a second (global) minimum at a large value of the scalar field of order $\Lambda$ [15]. Thus new physics must enter at a scale $\Lambda$ or below in order that the true minimum of the theory correspond to the observed $\mathrm{SU}(2) \otimes \mathrm{U}(1)$ broken vacuum with $v=246 \mathrm{GeV}$. Thus, given a value of $\Lambda$, one can compute the minimum and maximum Higgs boson mass allowed. The results of this computation (with shaded bands indicating the theoretical uncertainty of the result) are illustrated in Figure 1.2. 

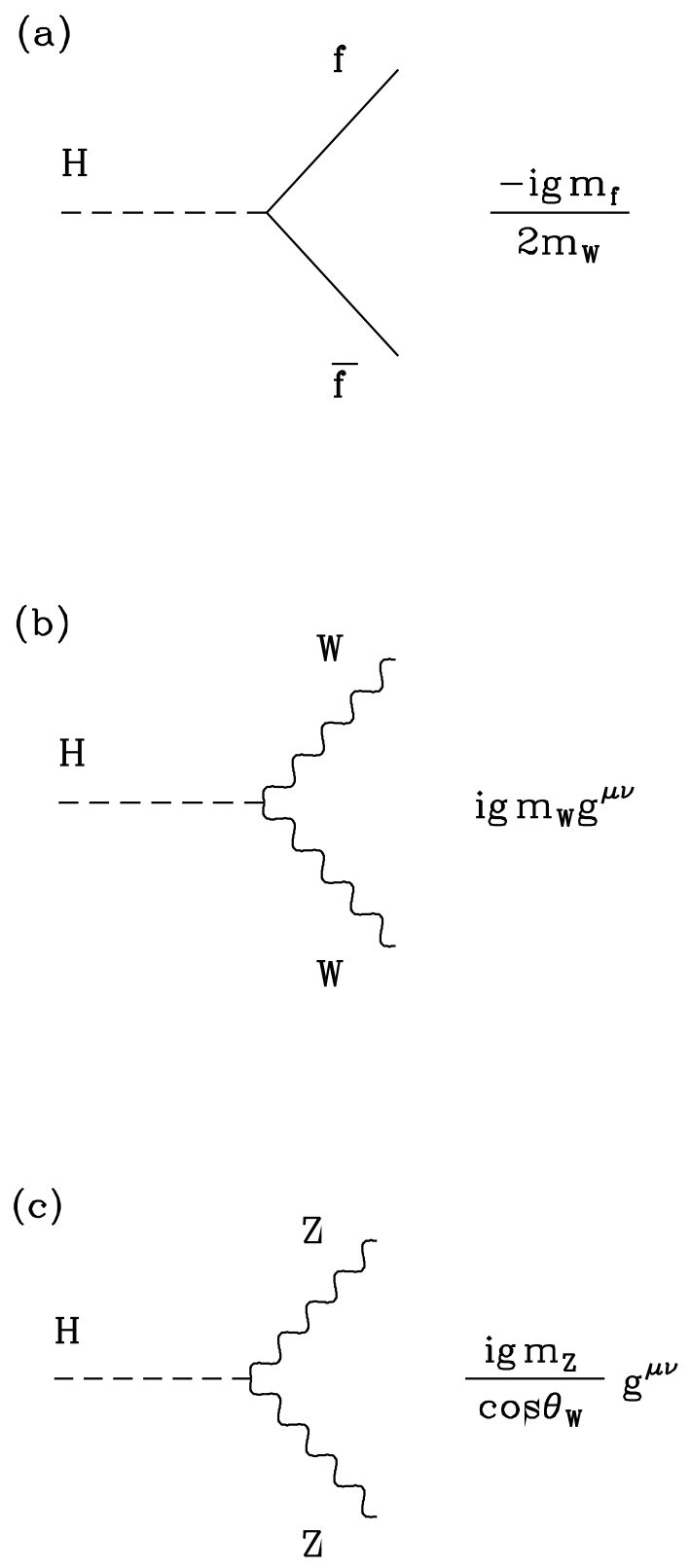

Figure 1.3: Standard Model Higgs boson interactions at tree-level

\subsection{The Standard Model Higgs Boson Search}

In the Standard Model, the Higgs boson mass is given by $M_{\text {Higgs }}=\sqrt{\lambda v^{2}}$, where $\lambda$ is the Higgs boson self-coupling parameter. And the value of the Standard Model Higgs boson mass is not predicted. However, other theoretical considerations, discussed 


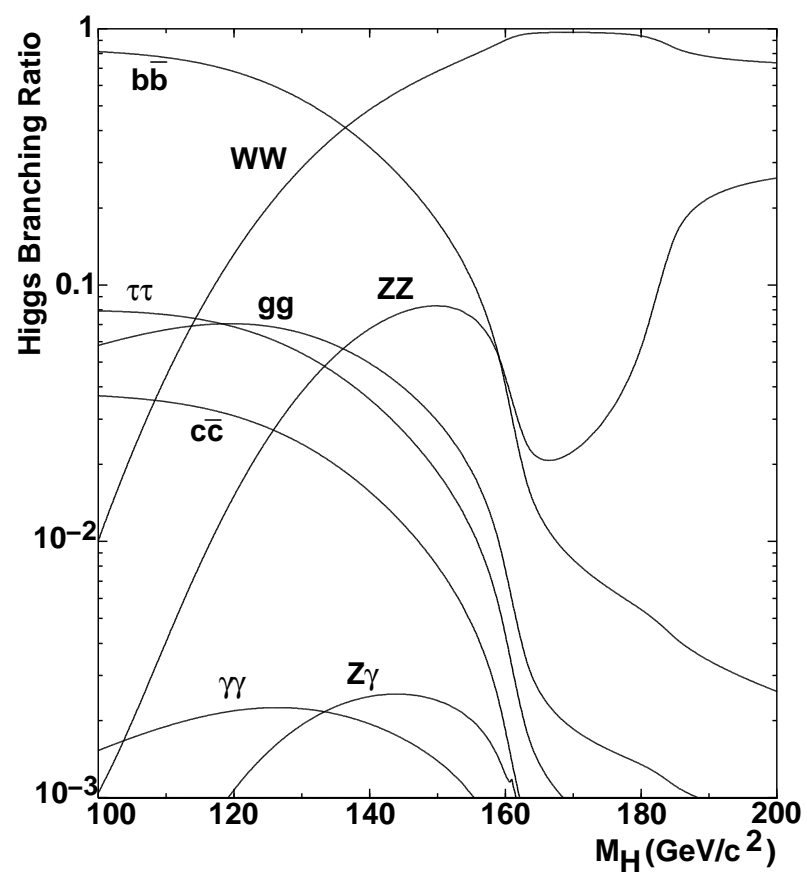

Figure 1.4: Branching ratios of the dominant decay modes of the Standard Model Higgs boson [17]. These results have been obtained with the program HDECAY [19], and include QCD corrections beyond the leading order.

in the previous section, place constrains on the Higgs boson mass as exhibited in Figure 1.2. In contrast, the Higgs boson couplings to fermions and gauge bosons are predicted by the theory. In particular, the Higgs boson couplings are proportional to the corresponding particle masses, as shown in Figure 1.3. The vertices of Figure 1.3 govern the most important features of Higgs boson phenomenology at colliders. In Higgs boson production and decay processes, the dominant mechanisms involve the coupling of the Higgs boson to the $W^{ \pm}, Z$ and the third generation quarks and leptons. It should be noted that a $g g H$ ( $g=$ gluon) coupling is induced by a one-loop graph in which the Higgs boson couples to a virtual $t \bar{t}$ pair. Likewise, a $H \gamma \gamma$ coupling is generated, although in this case the one-loop graph in which the Higgs boson couples to a virtual $W^{+} W^{-}$pair is the dominant contribution.

\subsubsection{Standard Model Higgs Boson Decay Modes}

The branching ratios for the dominant decay modes of a Standard Model Higgs boson are shown as a function of Higgs boson mass in Figure 1.4.

$\underline{H \rightarrow f \bar{f}}$ 
For Higgs boson masses below about $130 \mathrm{GeV}$, the decay $H \rightarrow b \bar{b}$ dominates, which the decay $H \rightarrow \tau^{+} \tau^{-}$can also be phenomenologically relevant. These results have been obtained with the program HDECAY [19], and include QCD corrections beyond the leading order. The QCD corrections are significant for the $H \rightarrow b \bar{b}, c \bar{c}$ decay widths due to large logarithmic contributions. The dominant part of these corrections can be absorbed by evaluating the running quark mass at a scale equal to the Higgs boson mass. In order to gain a consistent prediction of the partial decay widths one has to use $\overline{\mathrm{MS}}$ mass, $\bar{m}_{Q}\left(M_{Q}\right)$, obtained by fits to experimental data. The evolution of $\bar{m}_{Q}\left(M_{Q}\right)$ to $\bar{m}_{Q}\left(M_{\text {Higgs }}\right)$ is controlled by the renormalization group equations for the running $\overline{\mathrm{MS}}$ masses. A recent analysis of this type can be found in ref [18].

$H \rightarrow g g$

Though one-loop suppressed, the decay $H \rightarrow g g$ is competitive with other decays in the relevant Higgs boson mass region because of the large top Yukawa coupling and the color factor. The partial width for this decay is primarily of interest because it determines the $g g \rightarrow H$ productions cross-section.

$H \rightarrow W W, Z Z$

For Higgs boson masses above about $110 \mathrm{GeV}$, the decay mode $H \rightarrow W W$, where at least one of the $W$ bosons is off-shell (denoted henceforth by $W W^{*}$ ) becomes relevant. Above $135 \mathrm{GeV}$, this is the dominant decay mode [20][21]. The corresponding Higgs boson branching ratio to $Z Z^{*}$ is less useful for the Tevatron Higgs boson search, while constituting the gold-plated mode for the Higgs boson search at the LHC [22] when both $Z$ bosons decay to electrons or muons.

\subsubsection{Standard Model Higgs Boson Production at Tevatron}

This section describes the most important Higgs boson production processes at the Tevatron. The relevant cross section are depicted in Figure 1.5 [23]. Combining these Higgs boson production mechanisms with the decays discussed in the previous section, one obtains the most promising signatures.

$q \bar{q} \rightarrow V^{*} \rightarrow V H$

Given sufficient luminosity, the most promising Standard Model Higgs boson discovery mechanism at the Tevatron for $M_{\text {Higgs }}<135 \mathrm{GeV} / c^{2}$ consists of $q \bar{q}$ annihilation into a virtual $V^{*}\left(V=W^{ \pm}, Z\right)$, where the virtual $V^{*} \rightarrow V H$ followed by $H \rightarrow b \bar{b}$ and the leptonic decay of the $V$. The cross-section for $q \bar{q} \rightarrow W^{ \pm} H$ (summed over both $W$ charge states) reaches values of $0.3-0.02 \mathrm{pb}$ for $M_{\text {Higgs }}=100 \sim 200 \mathrm{GeV} / c^{2}$ as shown in Figure 1.5. The corresponding $q \bar{q} \rightarrow Z H$ cross-section is roughly a factor of two lower over the same Higgs boson mass range. The QCD corrections to $\sigma(V H)$ coincide with those of the Drell-Yan process and increase the cross-sections by about $30 \%$ [24][25][26]. The theoretical uncertainty is estimated to be about $15 \%$ from the 


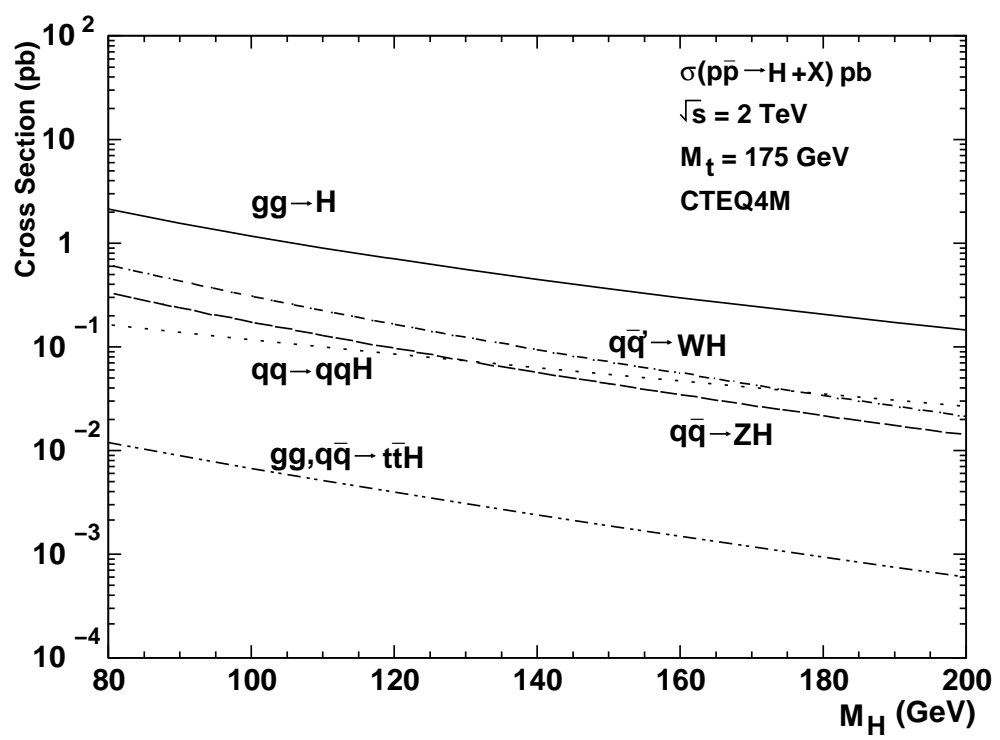

Figure 1.5: Higgs boson production cross-section (in units of pb) at the Tevatron for the various production mechanisms as a function of the Higgs boson mass, taken from refs [17]. The full NLO QCD-corrected results are shown for the gluon fusion $g g \rightarrow H$, vector boson fusion $q q \rightarrow V^{*} V^{*} q q \rightarrow H q q$, Higgs-strahlung processes $q \bar{q} \rightarrow V^{*} \rightarrow$ $V H$. Tree-level cross-section are exhibited for Higgs boson production processes in association with heavy quarks pairs: $g g, q \bar{q} \rightarrow t \bar{t} H$.

remaining scale dependence. The dependence on different sets of parton densities is rather weak and also leads to a variation of the production cross-sections by about $15 \%$.

The signature of Higgs boson production in the $V H$ channel are governed by the corresponding decays of the Higgs boson and vector boson. The dominant decay mode of the Higgs boson in the mass range of $M_{\text {Higgs }}<135 \mathrm{GeV} / c^{2}$ is $H \rightarrow b \bar{b}$; in this case, the leptonic decays of the final state $W$ and $Z$ (these include the missing energy signature associated with $Z \rightarrow \nu \nu$ ) serve as a trigger for the $V H$ events and significantly reduce QCD backgrounds. The detection of the Higgs boson signal via the more copious four-jet final states resulting from hadronic decays of $\mathrm{W}$ and $\mathrm{Z}$ is severely hampered by huge irreducible backgrounds. For the $M_{\text {Higgs }}>135 \mathrm{GeV} / c^{2}$ the Higgs boson decay mode $H \rightarrow W^{+} W^{-}$(where one $W$ is off-shell if $M_{\text {Higgs }}<2 M_{W}$ ) becomes dominant. In this case, the final state consists of three gauge bosons, $V W^{+} W^{-}$, and the like-sign dilepton signature becomes the primary signature for Higgs boson discovery. This is the signature of our analysis. 
$g g \rightarrow H$

The gluon fusion processes proceed primarily through a top quark triangle loop [27][28][29], and is the dominant neutral Higgs boson production mechanism at the Tevatron, with cross-sections of roughly $1.0-0.1 \mathrm{pb}$ for $M_{\text {Higgs }}=100 \sim 200 \mathrm{GeV} / c^{2}$, as shown in Figure 1.5. The two-loop QCD corrections enhance the gluon fusion cross-section by about $60-100 \%$ [30]. These are dominated by soft and collinear gluon radiation in the Standard Model [31]. The remaining scale dependence results in a theoretical uncertainty of about $20 \%$. The dependence of the gluon fusion cross-section on different parton densities yields roughly an additional 15\% uncertainty in the theoretical prediction. The signature $g g \rightarrow H \rightarrow b \bar{b}$ is not promising at the Tevatron due to the overwhelming QCD background of $b \bar{b}$ production. The $g g \rightarrow H \rightarrow \tau^{+} \tau^{-}$signature probably requires a high $\mathbb{E}_{T}$ resolution beyond the capabilities of the current detector. For $M_{\text {Higgs }}>135$ $\mathrm{GeV} / c^{2}$, the $H \rightarrow W^{+} W^{-}$decay channel becomes dominant and provides a potential Higgs boson discovery mode for the Tevatron. The strong angular correlations of the final state leptons resulting from $W W^{*}$ is one of the crucial ingredients for this discovery channel [32][33].

\section{$q \bar{q} \rightarrow q \bar{q} V^{*} V^{*} \rightarrow q \bar{q} H$}

The vector boson fusion is a shorthand notation for the full $q \bar{q} \rightarrow q \bar{q} H$ process, where the quark and anti-quark both radiate virtual vector bosons which then annihilate to produce the Higgs boson. The vector boson fusion via $u d \rightarrow d u H$ (and its chargeconjugate process) is also possible, although the relative contribution is small at the Tevatron. In Figure 1.5, all contributing processes are included, labeled $q q \rightarrow q q H$ for simplicity. The resulting Standard Model cross-sections are in the range $0.1-$ $0.03 \mathrm{pb}$ for $M_{\text {Higgs }}=100 \sim 200 \mathrm{GeV} / c^{2}$. The QCD corrections enhance the crosssection by about 10\% [26]. The modest vector boson fusion cross-section precludes observation of any of the rare SM Higgs boson decay modes in $q q \rightarrow q q H$ events at the Tevatron. For example, for $M_{\text {Higgs }}=120 \mathrm{GeV} / c^{2}$ and $30 \mathrm{fb}^{-1}$ of data, only six $H \rightarrow \gamma \gamma$ events are expected from the production process $q q \rightarrow q q H$. Similarly, under the same assumptions, only eleven dilepton events resulting from $H \rightarrow \tau^{+} \tau^{-}$are expected from the same data sample prior to any acceptance cuts that are required to reduce the large $Z \rightarrow \tau^{+} \tau^{-}, W^{+} W^{-}$or $t \bar{t}$ backgrounds. Typically these cuts reduce Higgs boson signal by another order of magnitude or more.

\section{5 "Bosophilic" Higgs Boson}

The Standard Model Higgs boson is responsible for generating the masses of both the weak vector bosons and the fermions. One can imagine that the mass generation of the weak vector bosons has little or nothing to do with that of the fermions. A Higgs boson associated only with the generation of the weak-vector-boson masses would be 

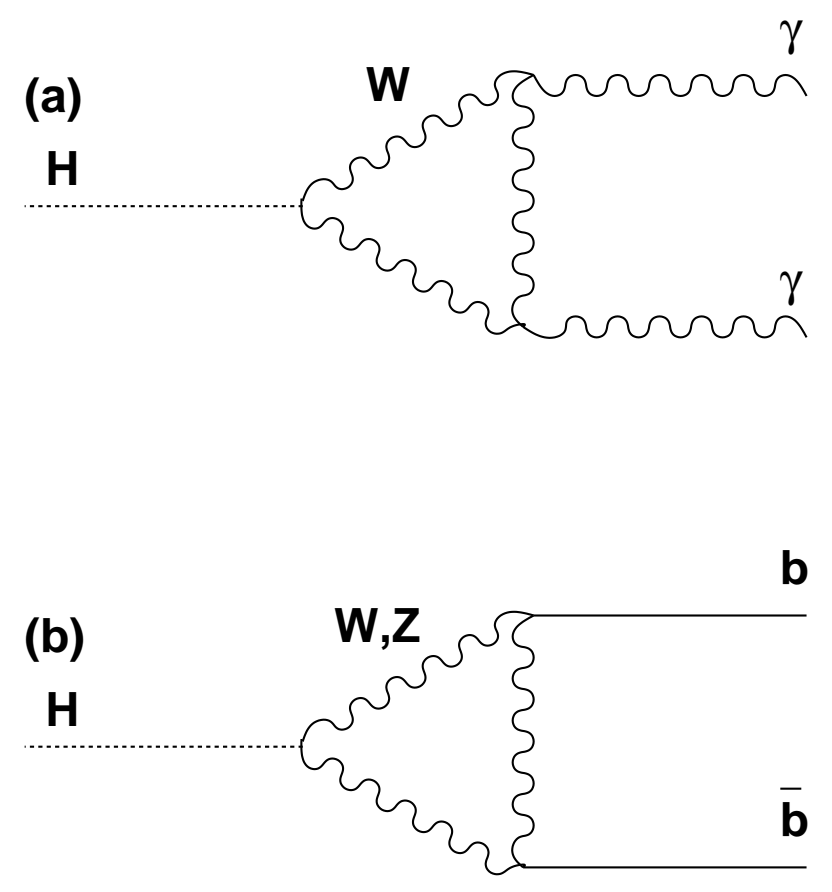

Figure 1.6: (a) Two-photon decay of a bosophilic Higgs boson via a $W$ boson loop; (b) decay of a bosophilic Higgs boson to $b \bar{b}$ via virtual $W$ and $Z$ bosons. The latter decay mode is suppressed relative to the former by $m_{b}^{2} / M_{W}^{2}$.

expected to have couplings to the weak vector bosons of Standard Model strength, but suppressed coupling to fermions. We will refer to such a particle as a "bosophilic" or "fermiophobic" Higgs boson [34]. For example, a bosophilic Higgs boson can arise in models with two Higgs doublets or with doublets and triplet. Since the fermionic decay modes of a bosophilic Higgs boson are greatly suppressed, the decay of a bosophilic Higgs boson of mass less than $2 M_{W}$ is not dominated by $H \rightarrow b \bar{b}$. The dominant decay mode of a sufficiently light bosophilic Higgs boson is to two photons via a $W$ boson loop as shown Figure 1.6(a). The bosophilic Higgs boson can also decay to $b \bar{b}$ at one loop, as shown in Figure 1.6(b); however, this decay mode is suppressed relative to the two-photon mode by $m_{b}^{2} / M_{W}^{2}$, and can be neglected. As the Standard Model Higgs boson the decay $H \rightarrow W W^{*}$ begins to compete with the two-photon decay. The branching ratios of a bosophilic Higgs boson decay to $\gamma \gamma, W W^{*}, Z Z$, and $\gamma Z$ are shown in Figure 1.7.

A bosophilic Higgs boson of mass less than about $108.2 \mathrm{GeV}$ would have been seen at LEP via $Z \rightarrow Z H$, with $H \rightarrow \gamma \gamma$ [35]. For the Tevatron this type of Higgs boson have been seen to less than $82 \mathrm{GeV}$ via $V \rightarrow V H(V=W, Z)$, with $H \rightarrow \gamma \gamma[36]$. 


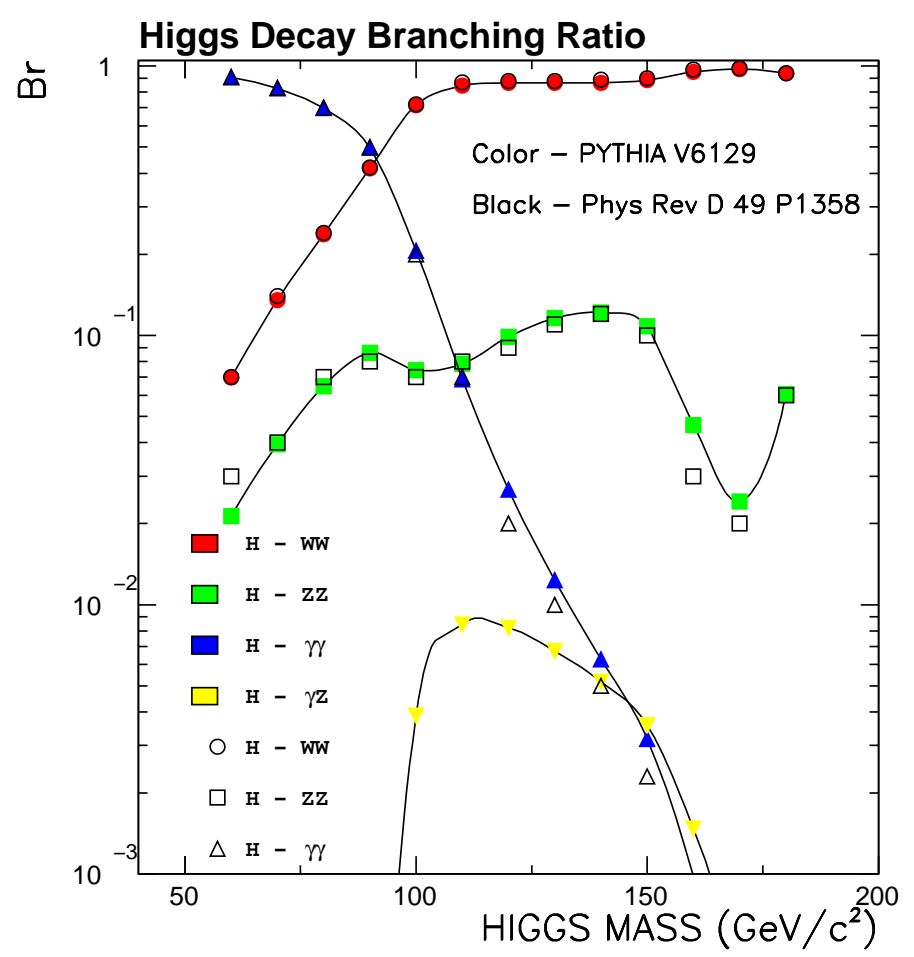

Figure 1.7: Branching ratios of a bosophilic Higgs boson decay to $\gamma \gamma, W W^{*}, Z Z^{*}$, and $\gamma Z$, versus the Higgs boson mass. 


\subsection{Physics Motivation}

Our physics objective is to search for the neutral Higgs boson using events containing a like-sign dilepton pair in the following reaction:

$$
q \bar{q}^{\prime} \rightarrow W^{ \pm} H \rightarrow W^{ \pm} W^{(*)} W^{(*)} \rightarrow \ell^{ \pm} \ell^{ \pm}+X .
$$

The relevant Higgs boson mass region is above $160 \mathrm{GeV} / c^{2}$ for the Standard Model Higgs boson where the branching fraction of $H \rightarrow W W^{*}$ supersedes that of $H \rightarrow b \bar{b}$. The search for this signature in the region at low mass is, however, still important because we need to investigate various Higgs boson couplings as an essential test to convince that signals are attributed to the Higgs boson production as we expect. This channel also covers the case beyond the Standard Model that the Higgs boson couples only to the gauge bosons, which is referred to as the bosophilic or fermiophobic Higgs boson. Such a case is interesting on its own from the view point of experimental physics if we consider the possibility that the mass origins could be different between gauge bosons and fermions. Theoretically, the bosophilic Higgs [34] boson appears, for example, in the two Higgs doublet model (type I). The production cross section is usually dependent on theoretical parameters such as $\tan \beta$, the ratio between the two vacuum expectation values of the CP-even neutral Higgs bosons, but, as a benchmark scenario, we consider it to be the same as the Standard Model in this analysis. The corresponding mass region suitable to our signature is above $110 \mathrm{GeV} / c^{2}$ where the branching fraction of $H \rightarrow \gamma \gamma$ is overtaken by this channel. On the experimental side, the like-sign dilepton event is one of the cleanest signature in hadron collisions. This analysis exploiting such a distinctive signature is therefore expected to have a high potential of the sensitivity for the search of Higgs boson. 


\section{Chapter 2}

\section{Apparatus}

Fermilab is currently the only laboratory in the world which can produce top quarks. In this chapter we describe the accelerators which make this possible, and the Collider Detector at Fermilab (CDF), which allows us to study the physics.

\subsection{Fermilab Run II Accelerator Complex}

Run II at Fermilab consists of $p \bar{p}$ collisions at a center-of-mass energy of $1.96 \mathrm{TeV}$. Figure 2.1 provides a pictorial overviews of the complex process which ultimately produces these collisions. Following this diagram, we describe the creation and acceleration of the proton and antiproton beams in the subsections below [37] [38].

\subsubsection{Proton Beam}

The creation of a proton beam begins with ordinary hydrogen gas $\left(H_{2}\right)$. The hydrogen molecules are split electrostatically within a cesium-walled chamber, which some of them become ionized due to the low work function of cesium. The resulting $H^{-}$ions undergo their first stage of acceleration in a Cockcroft-Walton [42], where is a simple capacitor-diode voltage multiplying array. The Cockcroft-Walton accelerates the $\mathrm{H}^{-}$ ions electrostatically to approximately $750 \mathrm{keV}$, where they enter a transfer section as continuous beam. The transfer section imparts a bunch structure to the beam, and injects it into the first stage of the Fermilab linear accelerator (linac), a standing-wave "Alvarez drift tube" linac [43]. The first section accelerates the ions to $116 \mathrm{MeV}$, where they pass through another coupling section and into the second stage, a $400 \mathrm{MeV}$ sidecoupled cavity linac. Upon exiting the linac, the ions pass through a thin carbon foil which removes the electrons. The beam (now composed only of protons) enters the booster, a 150 meter diameter synchrotron, which uses a multi-turn injection process since the time per revolution of a bunch $(2.2 \mu \mathrm{s})$ is shorter than the pulse length 


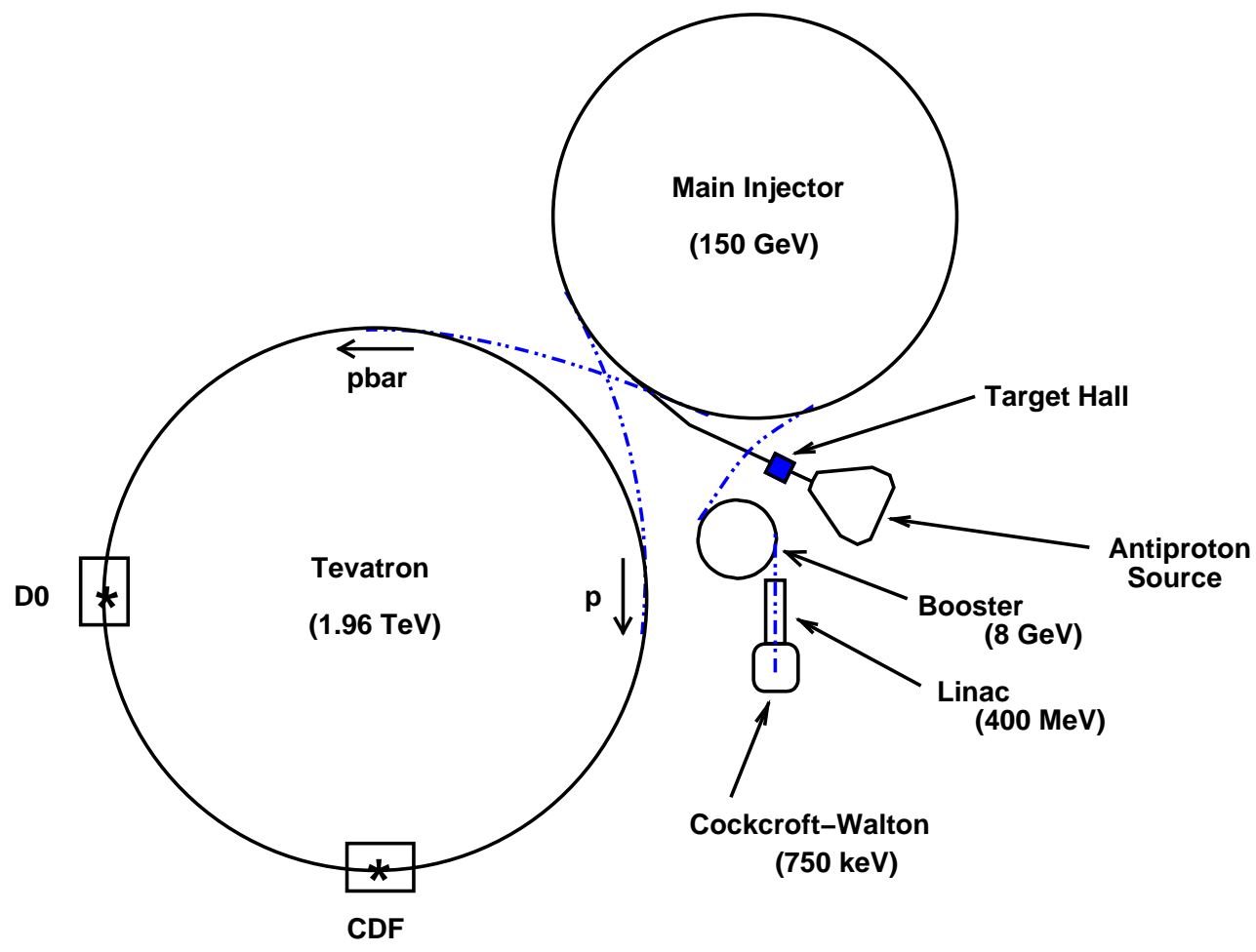

Figure 2.1: Pictorial overview of the Fermilab accelerator complex for Run II.

of the linac $(20 \mu \mathrm{s})$. The booster accelerates the proton beam to $8 \mathrm{GeV}$ in a few hundredths of a second. From the booster, the proton beam is transferred to the Main Injector (MI), which was a major part of the Run II upgrade to the Fermilab accelerator complex. In its collider injection mode, the MI (also a synchrotron) accelerates the proton beam to $150 \mathrm{GeV}$ in a few seconds, and performs coalescing and cogging of the beam in preparation for injection into the Tevatron. The Tevatron is a superconducting synchrotron, approximately four miles in circumference, which accelerates the proton beam to its final energy of $980 \mathrm{GeV}$.

\subsubsection{Antiproton Beam}

The creation of an antiproton beam is much more difficult, and begins with the Main Injector. In its antiproton mode the MI accelerates protons to $120 \mathrm{GeV}$ and directs them to a nickel target, creating a spray of particles which contains a small number of antiprotons. The particles are produced into a large solid angle, and must be focused in order to be collected. The focusing requirements are severe: The particles pass through a tubular piece of lithium which is made to support a current of $650 \mathrm{kA}$ while they traverse it. This is known as the lithium lens, since the resulting magnetic 
field provides focusing which is everywhere directed radially inward. Upon exiting the lens, the particles are deflected by a pulsed magnet which selects antiprotons and other negatively charged particles. After a short distance the other particles decay, leaving a beam of antiprotons. These are debunched and stochastically cooled [44] to increase the density of the beam by a factor of $\sim 18,000$. When enough antiprotons are circulating in the accumulator ring, they are transferred back into the MI, accelerated, and injected into the Tevatron in the opposite direction as the protons.

\subsubsection{Colliding Beams}

In Run II the Tevatron operates with 36 on 36 bunch structure (protons on antiprotons), with 396 ns spacing between bunches. At two points around the ring ("B0" and "D0") the beams are focused by quadrupole magnets to achieve the highest luminosity within the detectors. The luminosity is given by:

$$
L=\frac{\gamma}{2 \pi} f_{0} N_{\bar{p}} N_{p} B \frac{H}{\beta^{*}\left(\epsilon_{p}+\epsilon_{\bar{p}}\right)},
$$

where $\gamma$ is the relativistic energy factor, $f_{0}$ is the revolution frequency, $N_{p}$ and $N_{\bar{p}}$ are the number of protons and antiprotons per bunch, $B$ is the number of bunches of each type, $\beta^{*}$ is the beta function at the center of the interaction region, $\epsilon_{p}$ and $\epsilon_{\bar{p}}$ are the proton and antiproton $95 \%$ normalized emittances, and $H$ is the "hourglass form-factor" due to the bunch lengths. Although the luminosity depends explicitly on other quantities, increasing the proton and antiproton bunch intensities is the most

direct way to increase luminosity. As of August 2004, the end date for the data set used in this analysis, the average peak luminosity was about $1.0 \times 10^{32} \mathrm{~cm}^{-2} \mathrm{sec}^{-1}$ per a store (Figure 2.2). 


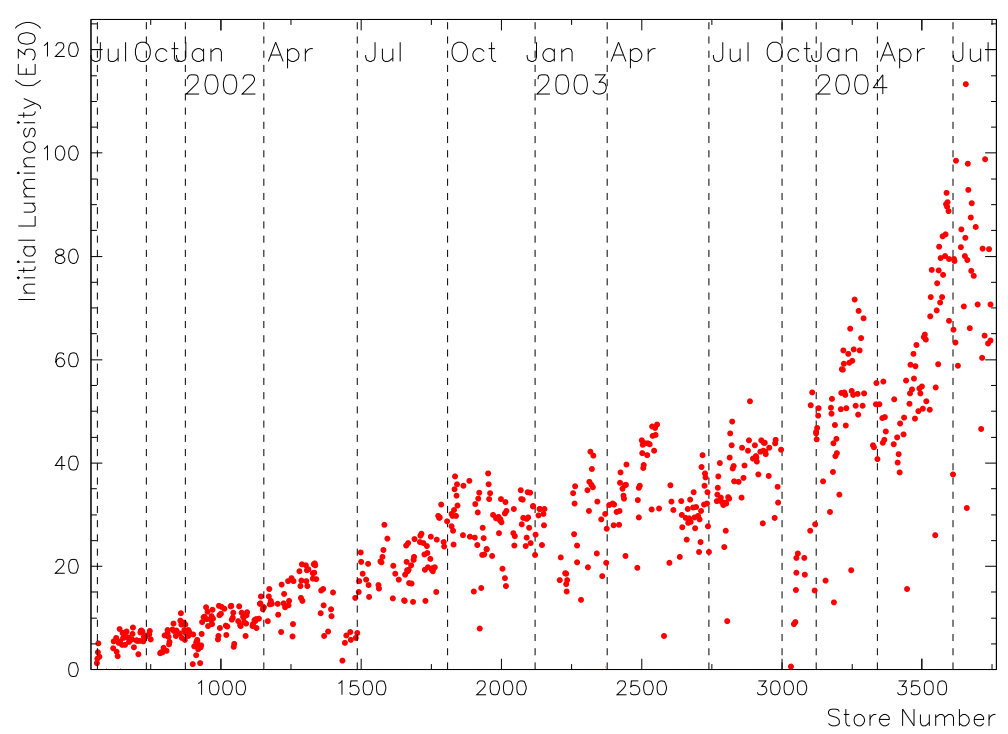

Figure 2.2: Initial instantaneous luminosity for each store.

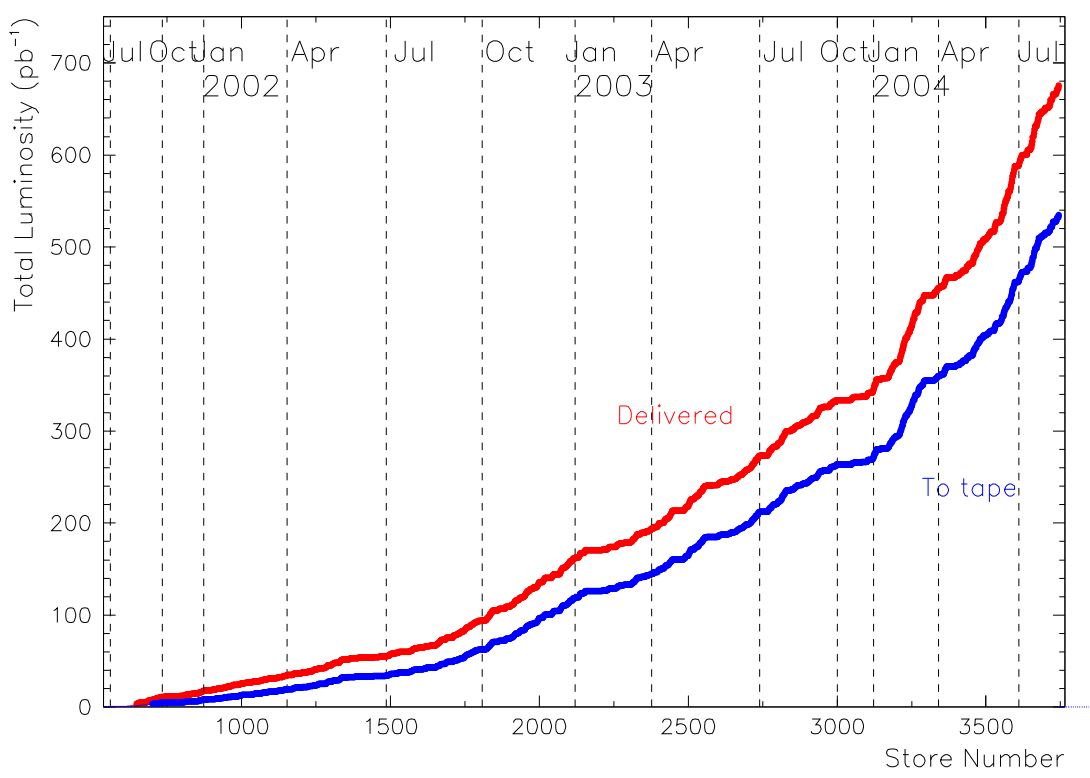

Figure 2.3: Integrated luminosity. Upper line is the delivered luminosity and lower one is the received luminosity. 


\subsection{Collider Detector at Fermilab}

The Collider Detector Fermilab (CDF) is a multi-purpose detector designed to study the physics of high-energy $p \bar{p}$ collisions [45]. CDF provides charged particle tracking in a solenoidal magnetic field, time-of-flight measurement, electromagnetic and hadronic calorimetry, and muon detection. An elevation view of the detector is shown in Figure 2.4 and 2.5. The overall geometry of the detector is cylindrical, with the Tevatron beamline running through the center, along the axis of symmetry. CDF employs a right-handed global coordinate system, with the origin at the interaction point in the very center of the detector. The set of coordinates used $(r, \phi, z, x, y$, and $\theta)$ is defined in Figure 2.6. The $p$ and $\bar{p}$ beams circulating in the Tevatron are unpolarized, and bunches exhibit a longitudinal density profile such that the resulting distribution of collisions along the beam axis is Gaussian, with a width of about $30 \mathrm{~cm}$. Since hadrons are composite objects, daughter particles from a $p \bar{p}$ collision are often produced with significant momentum along the $z$ axis. It is thus useful to define two variables, rapidity and pseudorapidity:

\section{Rapidity $y$}

The rapidity (not to be confused with Cartesian y in Figure 2.6) is defined as

$$
y=\frac{1}{2} \ln \left(\frac{E+p_{z}}{E-p_{z}}\right),
$$

where $E$ is the energy of a particle, and $p_{z}$ is the component of its momentum along the $z$ axis. The rapidity is additive under Lorentz boosts in the $z$ direction, and thus the rapidity differences are invariant under such boosts. Particle production is empirically observed to be essentially flat in the rapidity.

\section{Pseudorapidity $\eta$}

The pseudorapidity is defined as

$$
\eta=-\ln \left(\tan \frac{\theta}{2}\right),
$$

where $\theta$ is the polar angle measured from the $z$ axis, as shown in Figure 2.6. The pseudorapidity is equivalent to rapidity for massless particles, and is approximately equivalent for particles with momentum much greater than their rest mass. The pseudorapidity is experimentally convenient as a coordinate because the polar angle is easily measured within the detector.

Note that the detector is azimuthally symmetric about the $z$ axis, and mirror symmetric about the plane transverse to the beamline, centered at $z=0$. Similar to the Fermilab accelerator complex, CDF is a complex entity which is comprised of many subsystems; those most important are described in the following subsections, beginning nearest the interaction point and moving radially outward. 


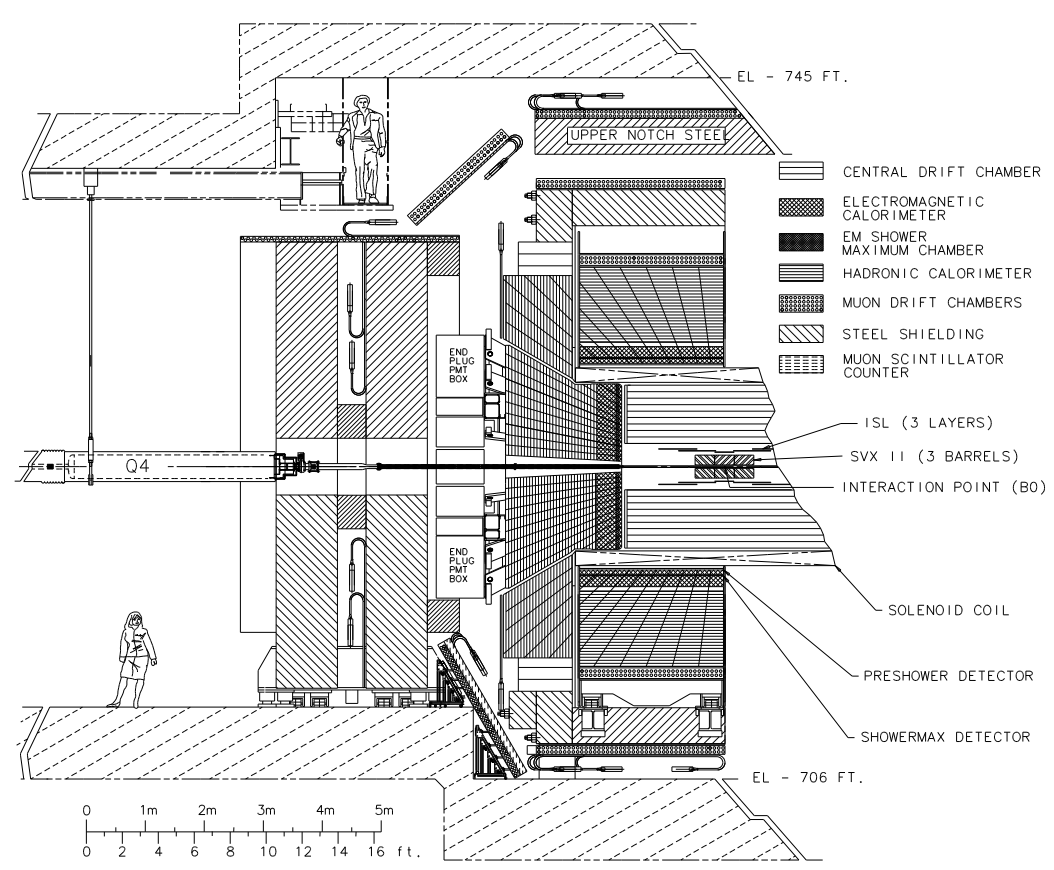

Figure 2.4: Longitudinal view of half of the CDF Run II detector.

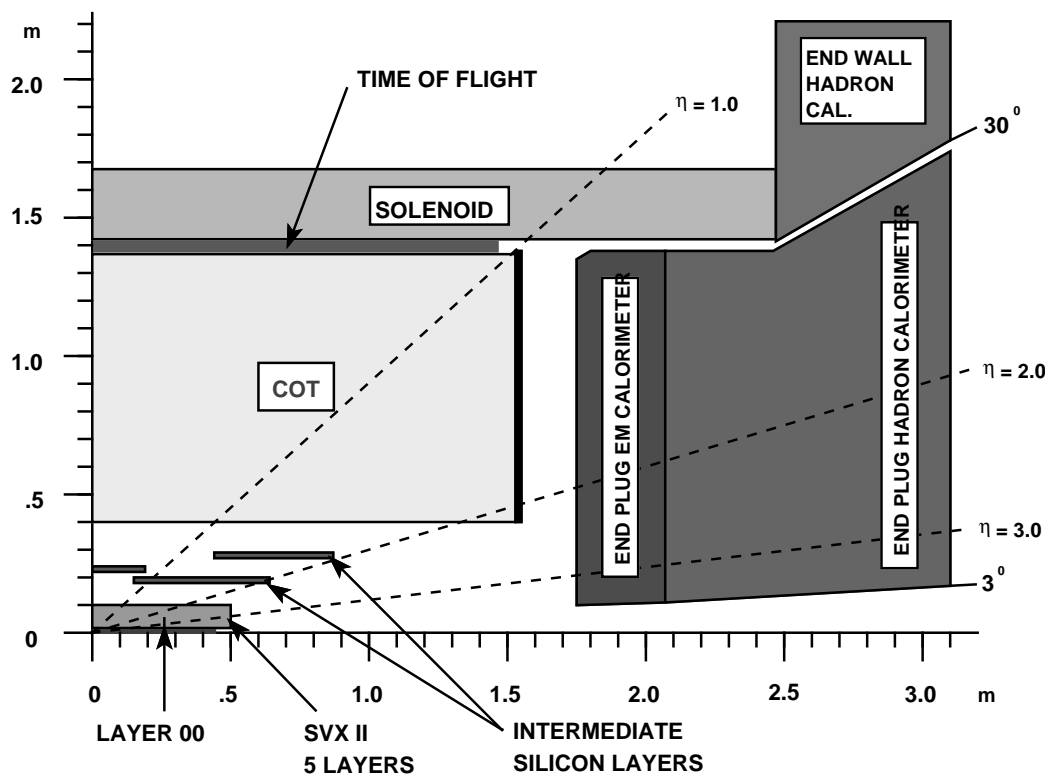

Figure 2.5: Longitudinal view of the CDF tracking system, representing a quarter of the whole detector. 


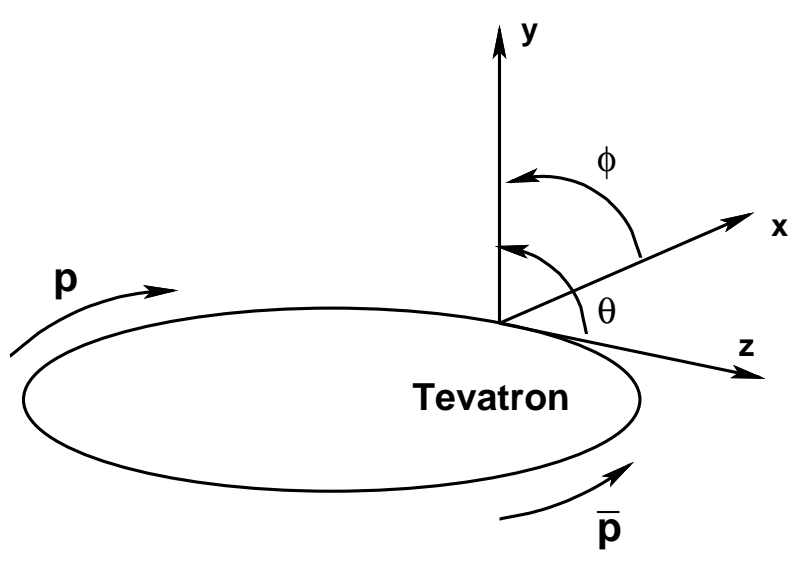

Figure 2.6: Coordinate system used at CDF

\subsubsection{Silicon Tracking Systems}

Silicon tracking for Run II at CDF is composed of three independent subsystems: A layer of single-sided silicon mounted directly on the beampipe (known as "Layer 00"), 5 layers of double-sided silicon located from $r=2.44 \mathrm{~cm}$ to $r=10.6 \mathrm{~cm}$ (known as "SVX II"), and 1+2 layers of double-sided silicon located at $r=20,22$, and 28 (known as "ISL"). The Layer 00 detector is not yet usable for physics analysis, and will not be described further here. The SVX (Silicon Vertex detector) is segmented into three independent barrels. Each barrel is $29 \mathrm{~cm}$ long and total length is $87 \mathrm{~cm}$ along the $z$ axis. Three of the layers combine the $r-\phi$ measurement on one side with a 90 degree stereo measurement on the other. The remaining two layers combine the $r-\phi$ measurements on one side, with a small stereo angle of 1.2 degree on the other. The stereo angle information from all the layers is combined to form a three dimensional track. A highly parallel fiber-based data acquisition system reads out the entire detector in approximately $10 \mu \mathrm{s}$. Figure 2.7 shows a three-dimensional drawing of the SVX. The ISL (Intermediate Silicon Layers) detector consists of three independent sections, a central layer $(r=22 \mathrm{~cm}, 0<|z|<25 \mathrm{~cm})$, a mid- $\eta$ layer $(r=20 \mathrm{~cm}$, $20<|z|<65 \mathrm{~cm})$, and a high- $\eta$ layer $(r=28 \mathrm{~cm}, 42<|z|<87.5 \mathrm{~cm})$. The structural and sensor designs for the SVX and ISL detectors are similar. A view of the SVX and ISL barrels are shown in Figure 2.8. Together, the SVX and ISL detector extend the reach of charged particle tracking at CDF to higher pseudorapidity. This is illustrated in Figure 2.5, which gives an overview of the designed tracking coverage for Run II. The silicon detectors also provide high spacial resolution near the interaction point, which is crucial for determining the precise location of vertices, including detached secondary vertices from $b$ quark decays. The ability to identify jets which come from $b$ quarks was of key importance in the Run I discovery of the top quark, and will be crucial to 
our ability to study the top sector with higher statistics in Run II.

The combined information of the SVX and ISL allows the reconstruction of three dimensional tracks independently of the COT (Central Outer Tracker), thus providing a tool to measure the efficiency of the latter. However, in this analysis only central leptons are used and no information from the silicon systems is used anywhere. The resolution of the SVX+ISL systems has been estimated by simulations to be

$$
\sigma_{p_{T}} / p_{T}^{2} \sim 0.4 \%[\mathrm{GeV} / c]^{-1} ; \quad \sigma_{d_{0}}=15 \mu \mathrm{m} ; \quad \sigma_{\phi_{0}}=0.3 \mathrm{mrad}
$$

where $d_{0}$ is the impact parameter of the track and $\phi_{0}$ is the angle that the track forms with the $x$-axis. 

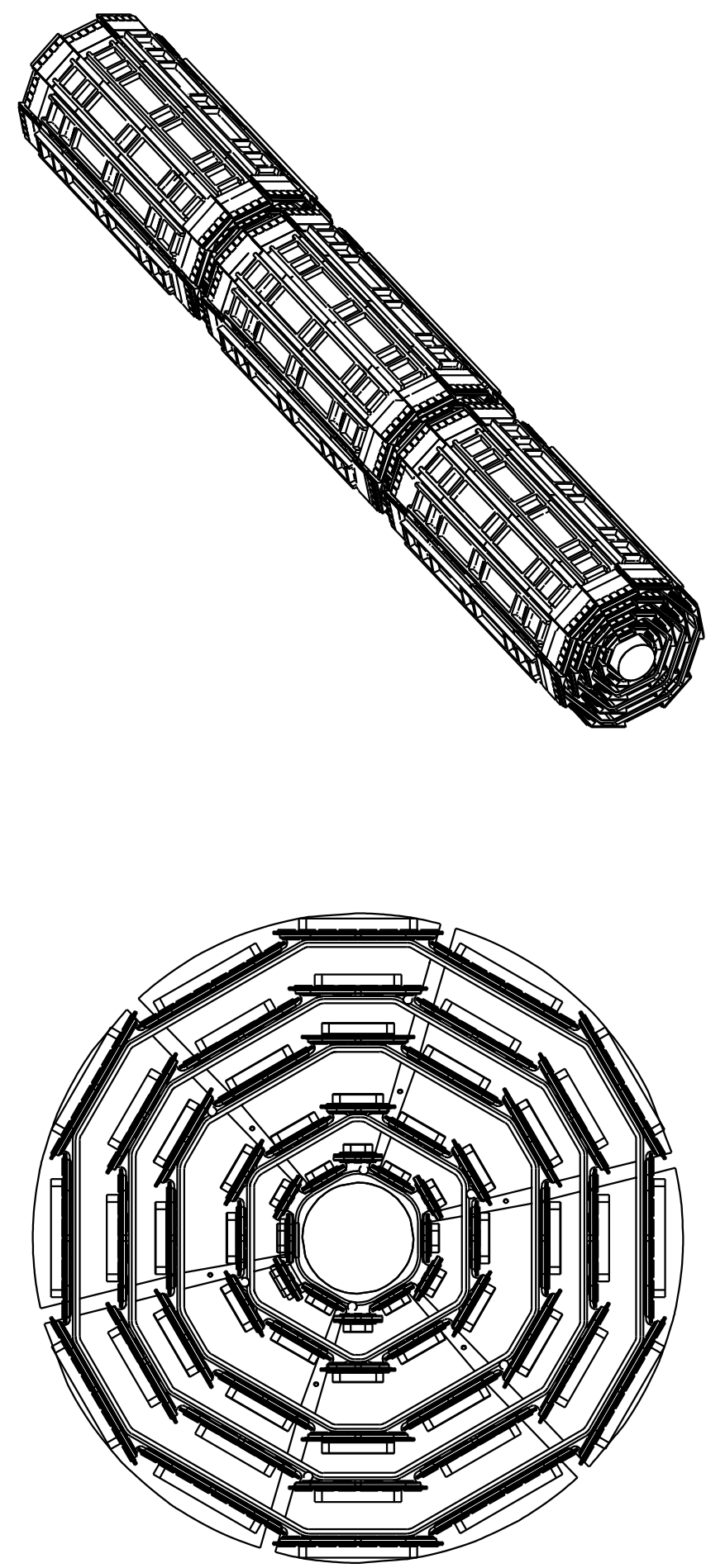

Figure 2.7: Upper figure is a view of the three barrels of SVX II silicon detector. Lower one is a view of one barrel. One barrel has 12 wedges and 5 layers. 


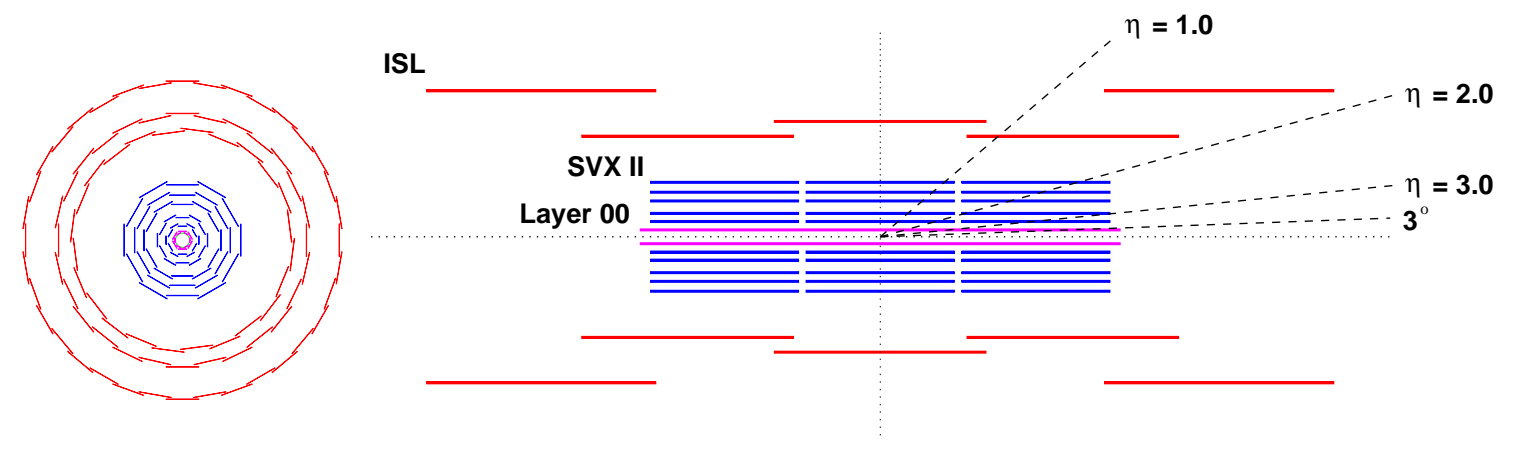

Figure 2.8: The CDF Silicon detectors (Layer 00 (Pink), SVX II(Blue), and ISL(Red)) end view showing the layering and space frame used to mount the detector. 


\subsubsection{Central Outer Tracker}

Tracking in the central region is provided by the Central Outer Tracker, an open cell drift chamber which consists of eight superlayers (Figure 2.9) of cells placed between the radii of $40 \mathrm{~cm}$ and $132 \mathrm{~cm}$ from the beam pipe. Each superlayer is composed of 12 layers of sense wires alternated with potential wires in a plane, as shown in Figure 2.9. The space between the cells is filled with a gas mixture of Argon and Ethane in the proportions 50:50, chosen to ensure a fast drift velocity $(\sim 100 \mu \mathrm{m} / \mathrm{ns})$ in order to deal with the expected high luminosity. Four of the superlayers are axial (for the measurements in the transverse plane) and the other four are stereo (for the $z$ measurements), with stereo angles of \pm 2 degree [46]; the superlayers are alternated starting with a stereo superlayer. A summary of the COT characteristics is given in Table 2.1. The ions produced by a charged particle passing through the COT are collected at the sense wires giving the $r-\phi$ information on the position of the hits. The hits from the stereo and axial wires are combined to obtain the $z$ position. The three-dimensional sequence of hits is fitted to form a track. The track momentum and charge are determined from the curvature in the magnetic field. If $B$ is the strength of the magnetic field, the transverse momentum $p_{T}$ of the track can be obtained by the relationship

$$
p_{T}=B q r,
$$

where $q$ is the charge of the particle and $r$ is the radius of curvature of the track. The resolution on the curvature has been studied using detailed simulation [45] and has been found to be $0.68 \times 10^{-4} \mathrm{~cm}^{-1}$ which corresponds to a momentum resolution $\sigma_{p_{T}} / p_{T}^{2} \sim 3 \times 10^{-3} \mathrm{GeV} / c^{-1}$. As more energetic tracks bend less, the curvature, and thus the momentum resolution of the COT, decreases for higher momentum tracks. The resolution on the impact parameter $d_{0}$ is about $600 \mu \mathrm{m}$, the resolution on $\cot \theta$ is $\sim 6 \times 10^{-3}$. In this analysis the momenta of the tracks associated with leptons are measured using the COT alone.

\subsubsection{Magnetic Field}

The CDF detector features a 1.41 Tesla axial magnetic field throughout the tracking volume (approximately $2.8 \mathrm{~m}$ and $3.5 \mathrm{~m}$ long), which enables measurements of charge and momentum via the tracking detectors. The field points in the $-z$ direction of the CDF global coordinate system, as shown in Figure 2.6. The solenoid coil is superconducting, and employs an aluminum-stabilized NbTi conductor. The normal operating field of 1.41 Tesla corresponds to a persistent current of 4,650 Amps. The conductor is cooled indirectly via liquid helium, and reinforced structurally by an external support cylinder made of aluminum. The infrastructure also includes an iron return yoke. Although the solenoid was commissioned in the 1980's and is subject to stresses during 

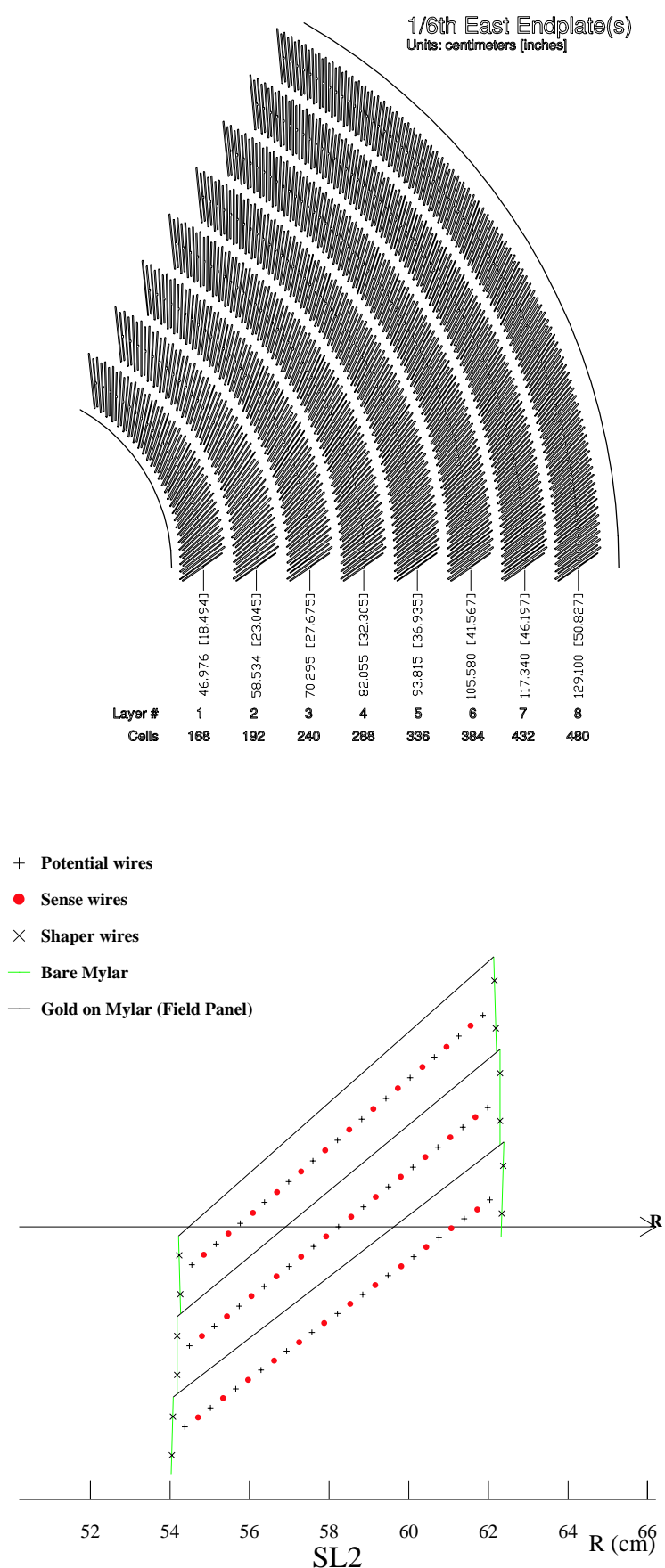

Figure 2.9: Upper figure is the endplate slots. In this figure the odd are stereo and the even are axial superlayers. Lower figure is a single cell layout. 


\begin{tabular}{cc}
\hline \hline \multicolumn{1}{c}{ COT } \\
\hline Radial coverage & 44 to $132 \mathrm{~cm}$ \\
Number of superlayers & 8 \\
Stereo angle (degree) & $+3,0,-3,0,+3,0,-3,0$ \\
Layers per superlayer & 12 \\
Drift field & $2.5 \mathrm{kV} / \mathrm{cm}$ \\
Maximum drift distance & $0.88 \mathrm{~cm}$ \\
Maximum drift time & $100 \mathrm{~ns}$ \\
Resolution per measurement & $180 \mu \mathrm{m}$ \\
Rapidity coverage & $|\eta|<1.0$ \\
Number of channels & 30,240 \\
Material thickness & $1.6 \% \mathrm{X}_{0}$ \\
\hline \hline
\end{tabular}

Table 2.1: Design parameters of the Central Outer Tracker at CDF.

every thermal cycle, it was conservatively built and is expected to last throughout Run II.

\subsubsection{Calorimetry}

The calorimeter systems at CDF surround the tracking volume and the solenoid, as shown in Figure 2.4 and 2.5, and provide energy measurement of electrons, photons, and jets. Each of the systems covers $2 \pi$ in azimuth $(\phi)$, a range in pseudorapidity $(\eta)$, and features an electromagnetic and a hadronic calorimeter. The calorimeters are segmented in $\eta$ and $\phi$ to form projective towers, which point back to the (nominal) interaction region at $z=0$. Towers exhibit a one-to-one EM:HAD matching geometry (with the exception of the two outer $\eta$ rings in the endplugs), such that the ratio of electromagnetic to hadronic energy can be measured for an individual tower.

\section{Central calorimeters}

The central calorimeters at CDF are denoted as CEM (Central Electromagnetic), CHA (Central Hadron), and WHA (Wall Hadron). For Run II these systems are essentially unchanged, receiving only an electronics upgrade. The CEM and CHA are constructed in wedges which span 15 degrees in azimuth and extend $\sim 250 \mathrm{~cm}$ in the positive and negative $z$ directions. There are thus 24 wedges on both the $+z$ and $-z$ sides of the detector, for a total of 48 wedges. A wedge contains ten towers, each of which covers a range in pseudorapidity of approximately $\Delta \eta=0.11$. This corresponds to a total span of $0<|\eta|<1.1$ for the CEM, and $0<|\eta|<0.9$ for CHA. Note that the central calorimeters have two phototubes per tower. The CEM is a lead-scintillator sampling calorimeter, approximately $19 X_{0}$ (radiation lengths) in depth, and has an energy 

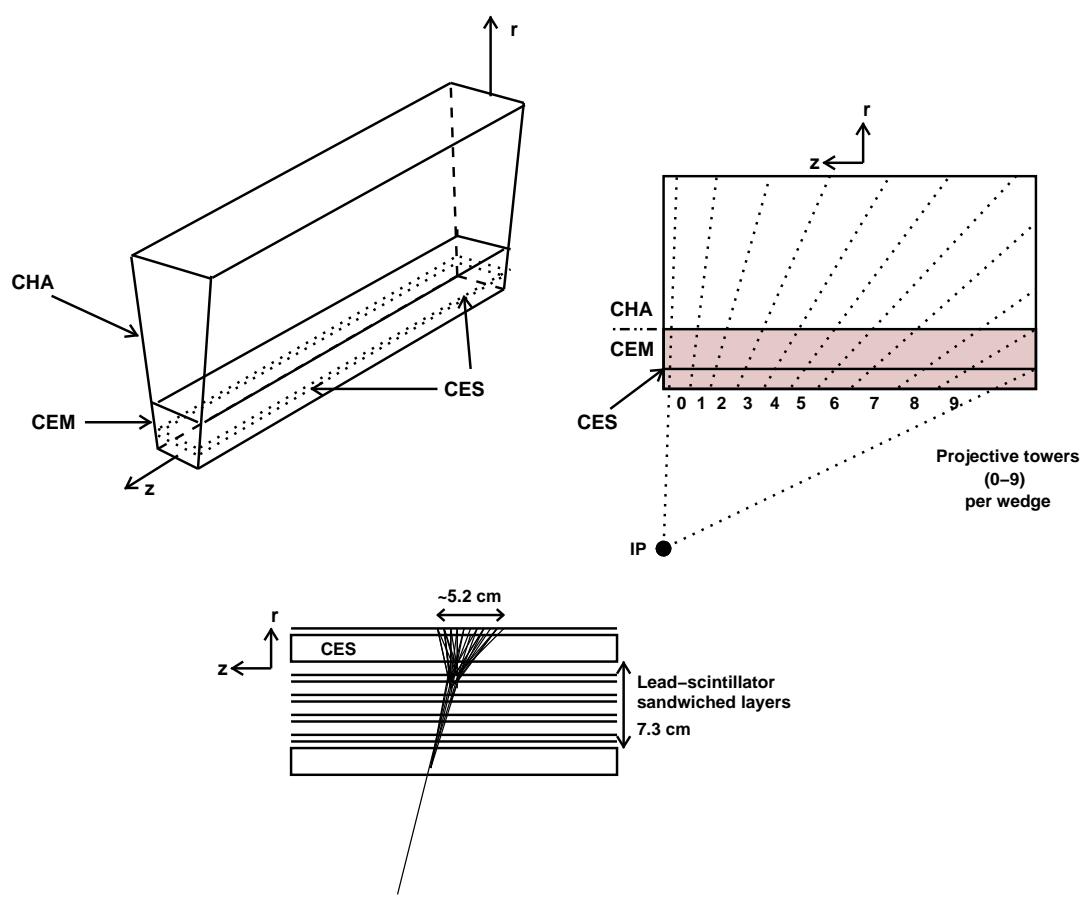

Figure 2.10: Top: Three-dimensional view of a central calorimeter wedge showing the location of each tower in pseudorapidity. Bottom: Illustration of the CES response to an EM shower.

resolution: $\sigma(E) / E=14.0 \% / \sqrt{E_{T}} \bigoplus 2 \%$, where $\bigoplus$ denotes addition in quadrature. At a depth of approximately $6 X_{0}$, the CEM contains a shower maximum detector called the CES. The CES employs proportional strip and wire counters in a finegrained array to provide precise position and shape information ( $\sim 2 \mathrm{~mm}$ resolution) for electromagnetic cascades. Figure 2.10 shows a three-dimensional view of a central calorimeter wedge and a close-up view of the CES.

The CHA is an iron-scintillator sampling calorimeter, approximately $4.5 \lambda$ (interaction lengths) in depth, and has an energy resolution of $\sigma(E) / E=50.0 \% / \sqrt{E_{T}} \bigoplus 3 \%$. The WHA is also an iron-scintillator sampling calorimeter, spanning a range in pseudorapidity of $0.7<|\eta|<1.3$. The WHA has a depth of $\sim 4.5 \lambda$, similar to the CHA, however it has worse energy resolution: $\sigma(E) / E=75.0 \% / \sqrt{E_{T}} \bigoplus 4 \%$.

\section{Endplug calorimeters}

The Run II upgrade at CDF included new scintillating endplug calorimeters. As in the central region, there is an electromagnetic calorimeter (PEM) with a shower maximum detector (PES), and a hadronic calorimeter (PHA). Figure 2.11 shows the layout of the detector and coverage in polar angle $(3<\theta<37$ degree). Each plug wedge spans 


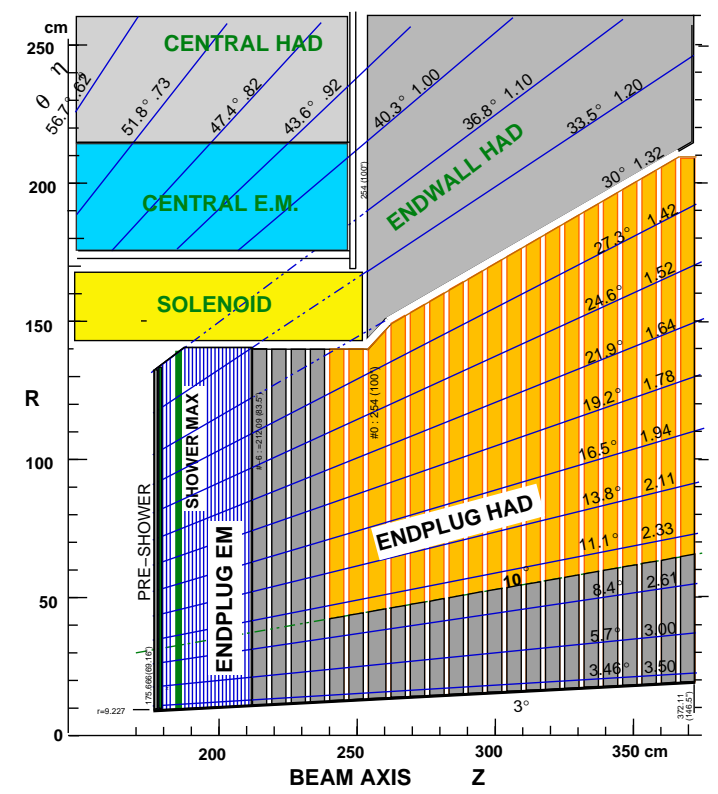

Figure 2.11: $r-z$ cross-section view of the upgraded Endplug Calorimeter of CDF.

15 degrees in azimuth. However from $1.1<|\eta|<2.1$ the segmentation in $\eta$ is doubled, and each tower spans only 7.5 degrees. Figure 2.12 shows the plug tower geometry. Note that, in contrast to the central region, each plug tower is read out by a single phototube. The PEM is a lead-scintillator sampling calorimeter, approximately $21 X_{0}$ in depth, and has an energy resolution of $\sigma(E) / E=16.0 \% / \sqrt{E_{T}} \bigoplus 1 \%$. The PHA is an iron-scintillator sampling calorimeter, approximately $7 \lambda_{0}$ in depth, and has an energy resolution of $\sigma(E) / E=80.0 \% / \sqrt{E_{T}} \bigoplus 5 \%$. The PES shower-maximum detector is located about $6 X_{0}$ deep within the PEM, and consist of of two layers (denoted "U" and "V") of scintillating strips. The strips are $5 \mathrm{~mm}$ wide, and roughly square in crosssection. The PES is segmented into 45 degrees sections, with strip in the U and V layers offset from the radial direction by +22.5 degree and -22.5 degree respectively. The position resolution of the PES is $\sim 1 \mathrm{~mm}$. There is also segmentation in pseudorapidity direction, low- $\eta$ region $(1.1<|\eta|<2.6)$ and a high- $\eta$ region $(2.6<|\eta|<3.5)$. Figure 2.13 shows a schematic layout of a PES 45 degree section. 


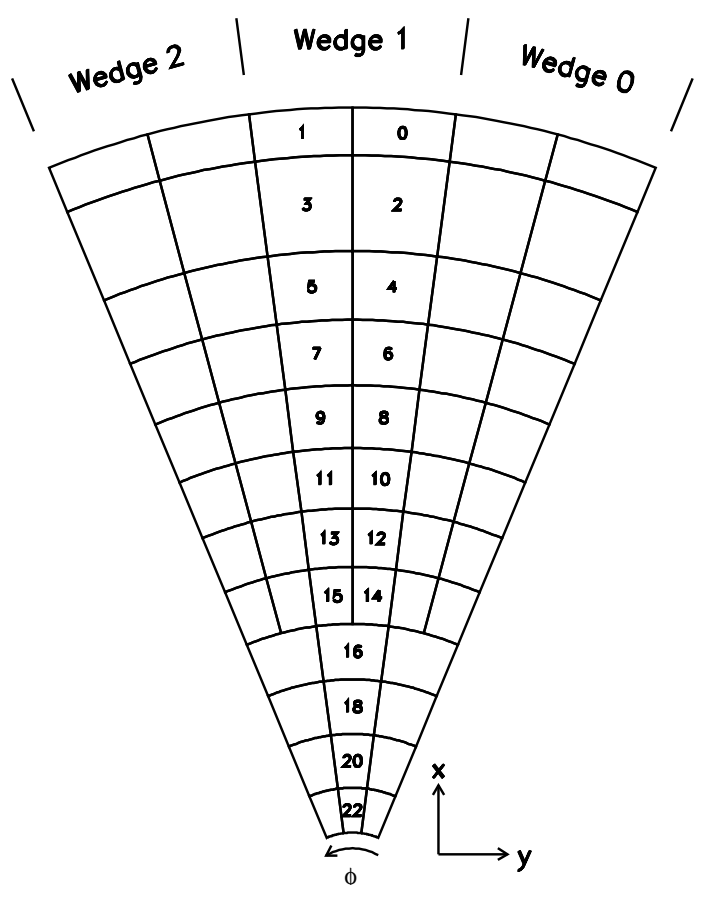

Figure 2.12: Tower geometry for the CDF run II Endplug calorimeters. Each wedge covers 15 degree in azimuth. As shown, towers 0 -14 span the region $1.1<|\eta|<2.1$, and towers 16-22 span the region $2.1<|\eta|<3.6$.

\begin{tabular}{cccccc}
\hline \hline Calorimeter & CEM & CHA & WHA & PEM & PHA \\
\hline Coverage & $|\eta|<1.1$ & $|\eta|<0.9$ & $0.7<|\eta|<1.3$ & $1.1<|\eta|<3.6$ & $1.1<|\eta|<3.6$ \\
Modules & 48 & 48 & 48 & 24 & 24 \\
$\eta$ towers per module & 10 & 8 & 6 & 12 & 10 \\
Layers & 31 & 32 & 15 & 23 & 23 \\
Material & Lead & Steel & Steel & Lead & Iron \\
Radiation Length & $19 \mathrm{X}_{0}$ & $4.5 \lambda_{0}$ & $4.5 \lambda_{0}$ & $21 \mathrm{X}_{0}$ & $7 \lambda_{0}$ \\
Energy Resolution & $14.0 \% / \sqrt{E_{T}}$ & $50.0 \% / \sqrt{E_{T}}$ & $75.0 \% / \sqrt{E_{T}}$ & $16.0 \% / \sqrt{E_{T}}$ & $80.0 \% / \sqrt{E_{T}}$ \\
& $\bigoplus 2 \%$ & $\bigoplus 3 \%$ & $\oplus 4 \%$ & $\oplus 1 \%$ & $\oplus 5 \%$ \\
\hline \hline
\end{tabular}

Table 2.2: Summary of the CDF calorimeters. The different components CEM, CHA, etc. are described in the text. 


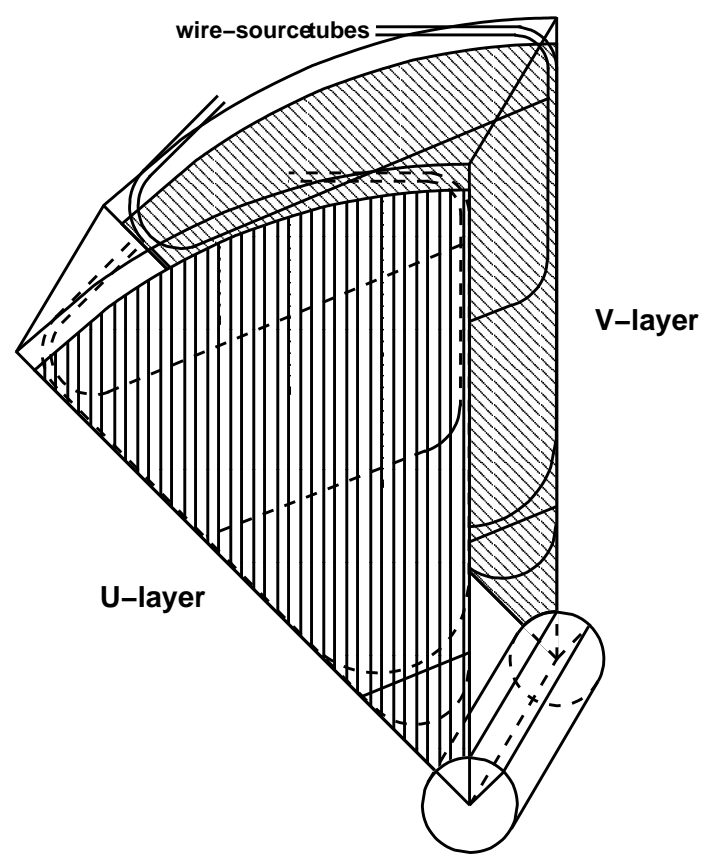

Figure 2.13: Schematic drawing of a 45 degree section of the PES detector. The PES is calibrated using a radioactive source, which traverses the indicated path during calibration and is retracted into a shielded box when not in use. 


\begin{tabular}{ccccc}
\hline \hline Muon system & CMU & CMP & CMX & BMU \\
\hline Pseudorapidity coverage & $|\eta|<0.6$ & $|\eta|<0.6$ & $0.6<|\eta|<1.0$ & $1.0<|\eta|<1.5$ \\
Drift tube length & $226 \mathrm{~cm}$ & $640 \mathrm{~cm}$ & $180 \mathrm{~cm}$ & $363 \mathrm{~cm}$ \\
Drift tube width & $6.35 \mathrm{~cm}$ & $15 \mathrm{~cm}$ & $15 \mathrm{~cm}$ & $8.4 \mathrm{~cm}$ \\
Maximum drift time & $800 \mathrm{~ns}$ & $1.4 \mu \mathrm{m}$ & $1.4 \mu \mathrm{m}$ & $800 \mathrm{~ns}$ \\
Number of drift tube & 2304 & 1076 & 2208 & 1728 \\
Scintillation counter thickness & N/A & $2.5 \mathrm{~cm}$ & $1.5 \mathrm{~cm}$ & $2.5 \mathrm{~cm}$ \\
Scintillation counter width & N/A & $30 \mathrm{~cm}$ & $30-40 \mathrm{~cm}$ & $17 \mathrm{~cm}$ \\
Scintillation counter lengths & N/A & $320 \mathrm{~cm}$ & $180 \mathrm{~cm}$ & $180 \mathrm{~cm}$ \\
Total counters & N/A & 269 & 324 & 864 \\
Pion interaction length & 5.5 & 7.8 & 6.2 & $6.2-20$ \\
Minimum detectable muon $p_{T}$ & $1.4 \mathrm{GeV} / c$ & $2.2 \mathrm{GeV} / c$ & $1.4 \mathrm{GeV} / c$ & $1.4-2.0 \mathrm{GeV} / c$ \\
Multiple scattering resolution & $12 \mathrm{~cm} / p$ & $15 \mathrm{~cm} / p$ & $13 \mathrm{~cm} / p$ & $13-25 \mathrm{~cm} / p$ \\
\hline \hline
\end{tabular}

Table 2.3: Design parameters of the CDF II Muon Detectors. Pion interaction lengths and multiple scattering are computed at a reference angle of $\theta=55$ degree in CMX, and show a range of values for the BMU.

\subsubsection{Muon Systems}

Electrons lose most of their energy in the EM calorimeters and hadrons in the hadron calorimeters. On the other hand, muons are known to penetrate the tracking systems and the calorimeters leaving very little energy. For this reason a muon candidate is created from minimum energy deposited in the calorimeters matched with a minimum ionizing track in the COT and with hits in the "muon chambers", placed outside the CDF detector. CDF uses four systems of absorbers, scintillators and proportional chambers in the detection of muons over the region $|\eta|<2.0$. They are the Central Muon detector (CMU), the Central Muon Upgrade detector (CMP), the Central Muon Extension detector (CMX), and the Barrel Muon detector (BMU). All four detectors are composed of layers of single wire drift chambers, of which alternating layers are staggered in order to eliminate hits position ambiguities (Figure 2.14). The creation of a muon object involves the process of forming a "stub" from hits in the muon chambers, and matching it to a COT track. An overview of their $\eta$ and $\phi$ coverages at CDF is shown in Figure 2.15, and their characteristics are briefly summarized in Table 2.3.

\subsubsection{Trigger Systems}

The trigger plays a crucial role in $p \bar{p}$ collider experiments. Since they usually have a high rate of collisions, much higher than the speed with which these events can be written to tape, it is essential that the trigger system be able to provide as much event 


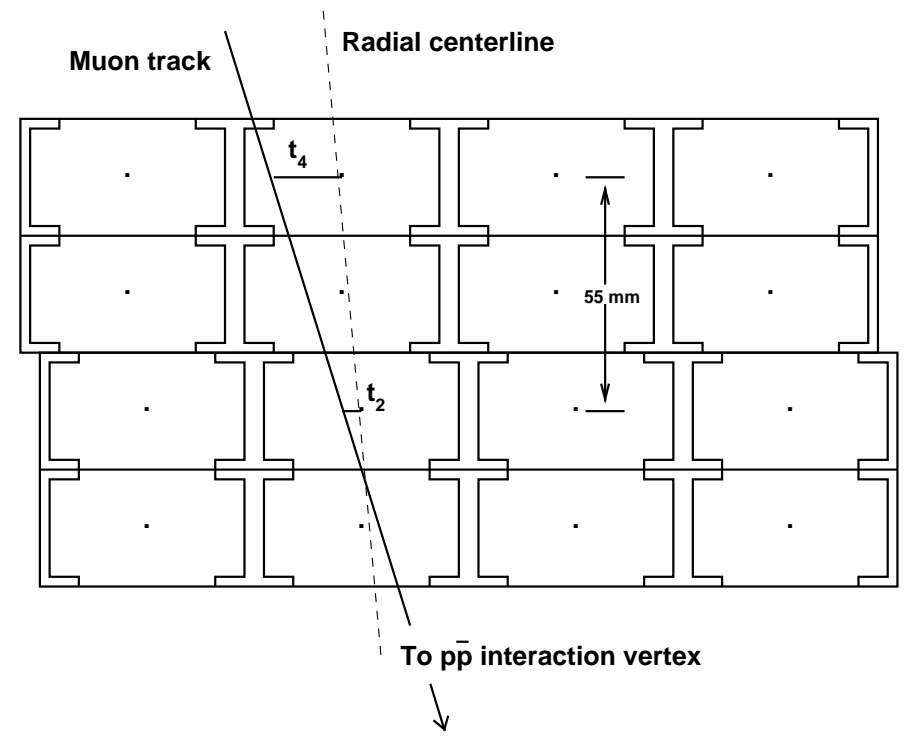

Figure 2.14: Cross-section view of drift tubes in a muon chamber. A stub is formed from the trajectory of a muon passing through the chamber, and the stub is matched to a track to become a reconstructed muon.

throughputs possible in the short time. In Run II the collision rate is, at nominal luminosity, essentially equal to the crossing-rate, $7.6 \mathrm{MHz}$, while the tape recording rate is less than $75 \mathrm{~Hz}$. The role of the trigger is efficiently select the most interesting events among the large amount of "minimum bias". Due to the improvements in the accelerator configuration all of the trigger system needed to be replaced in Run II to have a higher rejection factor with respect to Run I and still ensure the maximum event recording efficiency. The CDF trigger system has a three level architecture, with each level providing a rate of reduction sufficient to allow for processing in the next level with the minimum dead time. The three levels will be described separately in the following paragraphs. Figure 2.16 shows a data flow in the data acquisition system (DAQ system), while the block diagram of the trigger system is shown in Figure 2.17.

\section{Level-1}

The first level of trigger selection Level-1 (L1) uses custom designed hardware to find physics objects based on a subset of the detector information and then makes a decision based on simple counting of these objects. The input to the L1 hardware comes from the calorimeters, tracking chambers and muon detectors. The decision to retain an event for further processing is based on the number and energies of the electron, jet and muon candidates as well as the missing energy in the event, or on the kinematic properties of few of these objects. The L1 hardware consists of three parallel synchronous processing 


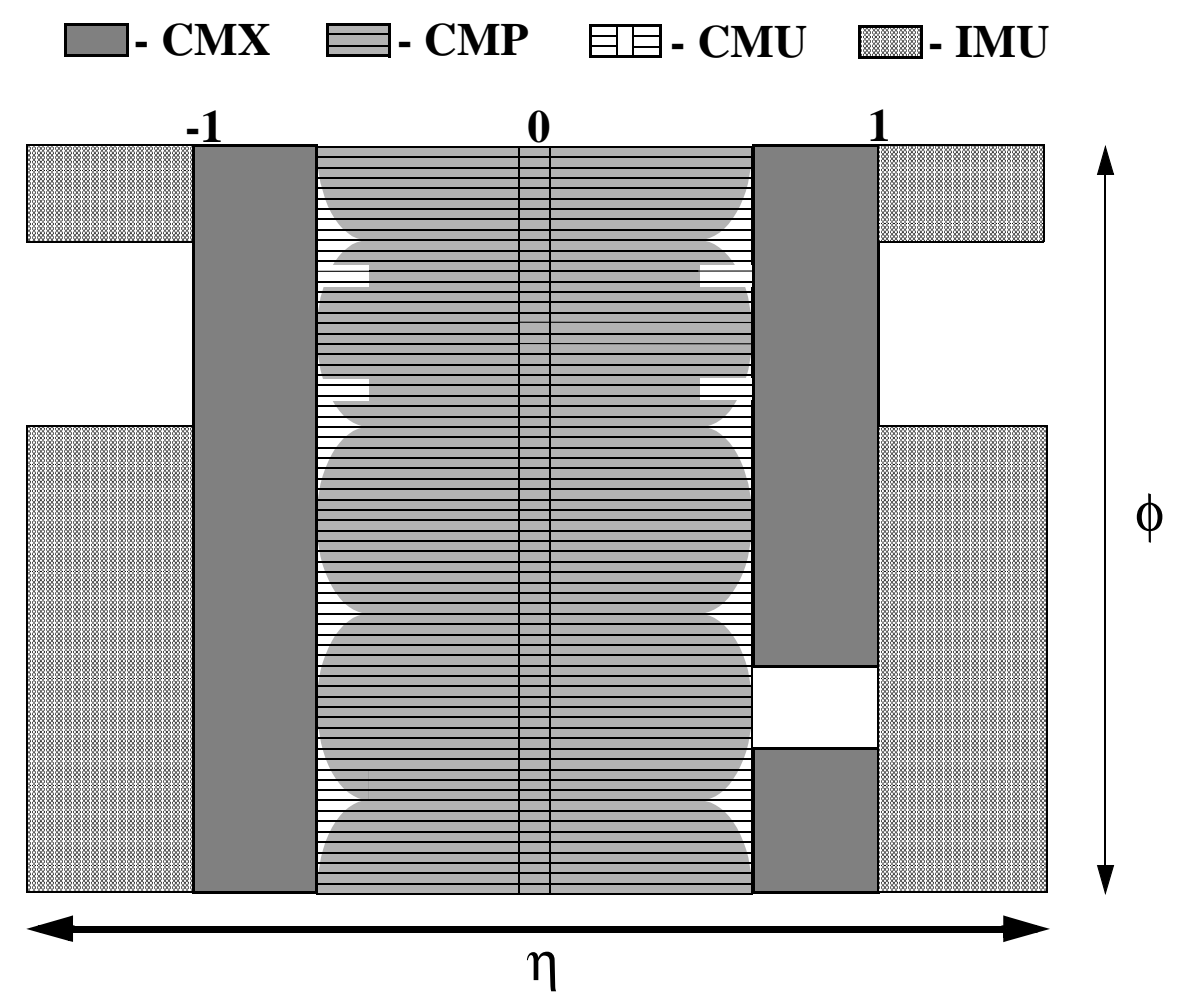

Figure 2.15: Map of the muon coverage for this analysis.

streams which feed inputs of the single Global Level-1 decision unit (Figure 2.17). One stream finds calorimeter objects (e.g. electrons and jets), another finds muons and the third finds tracks in the central region. The L1 trigger can be formed using these streams singularly as well as AND or OR combinations of them. All elements of the L1 trigger are synchronized to the same 132 ns clock, with a decision made every 132 ns by Global L1. In the period of data taking considered in this analysis the accelerator was operating in 36 bunches mode (396 ns) and the trigger was clocked every $132 \mathrm{~ns}$ with the two intermediate clock cycles automatically rejected. The maximum L1 accept rate is $20 \mathrm{kHz}$, while the typical one is about $12 \mathrm{kHz}$.

\section{Level-2}

Events accepted by L1 are processed by the second level of trigger Level-2 (L2), which is composed of several asynchronous subsystems. These provide input data to programmable L2 processors in the Global L2 crate, which determine if any of the L2 triggers are satisfied. Processing for a L2 trigger decision starts after the event is written into one of the four L2 buffers by a L1 accept. When L2 is analyzing the event in one of the buffers, that buffer cannot be used for additional L1 accepts. If all the 


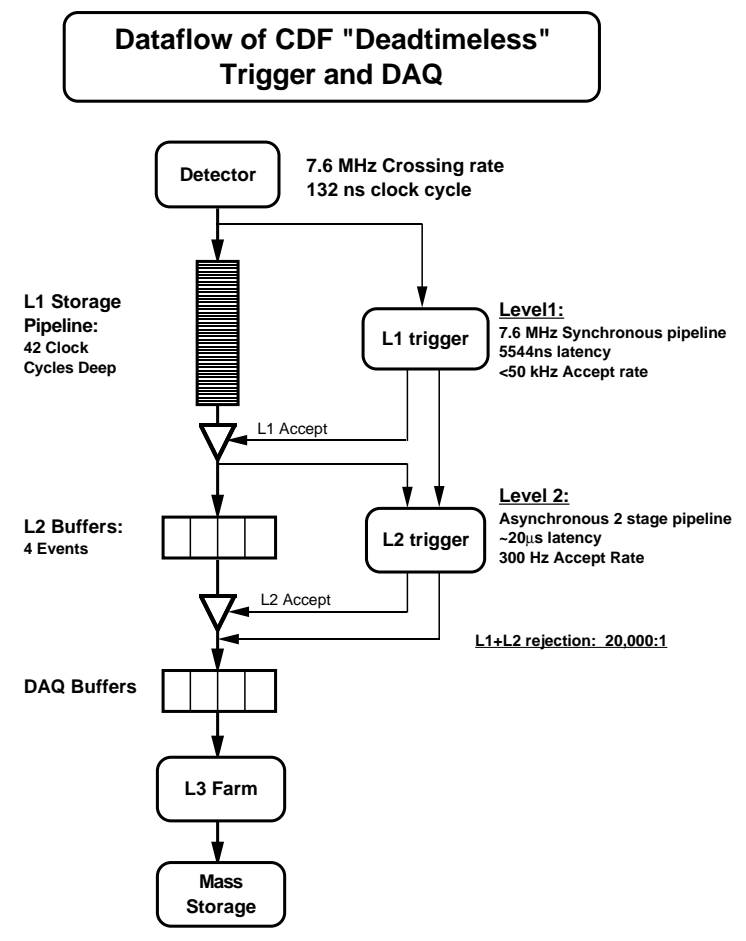

Figure 2.16: The three level deadtime-less trigger used to control DAQ of the CDF detector

four buffers are full, the deadtime of the data acquisition is increased. It follows that the time required for a L2 decision needs to be less than about $80 \%$ of the average time between L1 accepts in order to keep the deadtime as low as possible. For this purpose L2 has been pipelined into two stages each taking approximately $10 \mu \mathrm{s}$, which is sufficient to keep the deadtime at a minimum, even if L1 had an accept-rate of 50 $\mathrm{kHz}$. The L2 buffers perform a limited event reconstruction using essentially all the information used in L1, but with higher precision. In addition, at L2, data from the central shower-max detector and the SVX are available, which improve respectively the identification of electrons and photons and the reconstruction of the secondary vertices. Furthermore, a jet reconstruction algorithm is provided by the L2 cluster finder. After all of the data are stored in the processors, the event is examined to check if the criteria of any of the L2 triggers have been satisfied. This operation can be performed while the new events are being loaded into memory, thus not affecting the dead time. The typical L2 accept rate, as of this writing, is between 100 and $300 \mathrm{~Hz}$, depending on the initial luminosity.

\section{Level-3}

The Level-3 (L3) trigger subsystem is composed of two main components, the Event 


\section{RUN II TRIGGER SYSTEM}

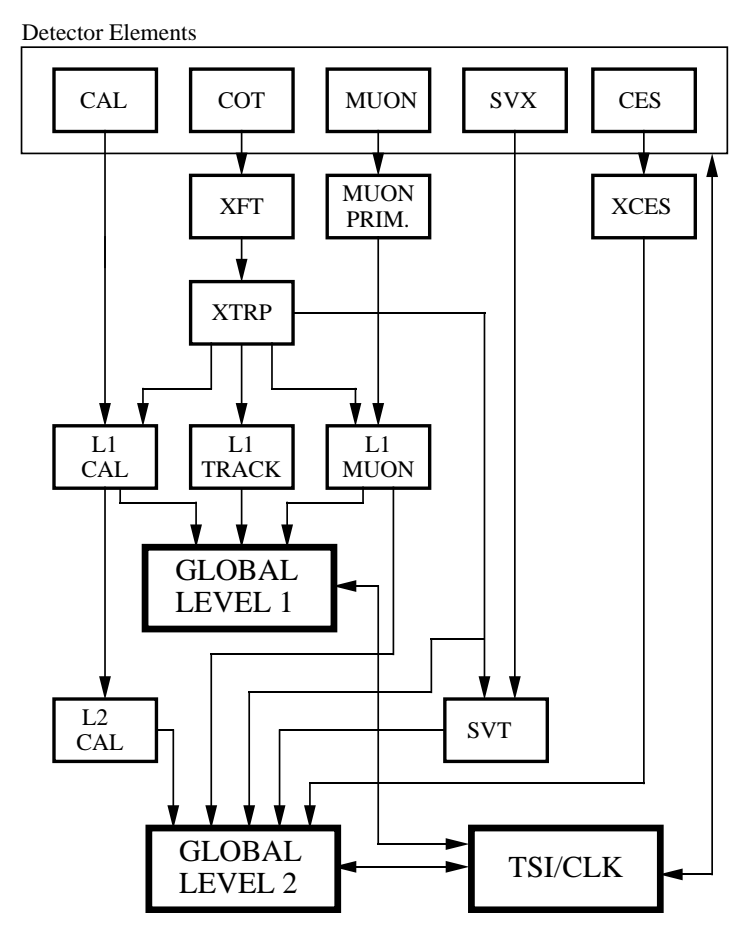

Figure 2.17: The readout functional block diagram. The acronyms have been already introduced in the text, apart from the following: XFT: Extremely fast tracker, XTRP: Track extrapolation module, SVT: SVX track processor in Level2 trigger system, TSI: Trigger System Interface.

Builder (EVB) and the Level-3 Farm. Level-1 and Level-2 systems need to make their decisions at a very high rate which makes it impossible to fully reconstruct each event. While Level-1 and Level-2 algorithms use small predefined pieces of event data to make their decision, the event pieces are stored in the buffers of the 140 Front End crates which constitute the EVB. After a L2 decision is made, the Event Builder assembles all event fragments from the Front End crates into one data block.

The 16 subfarms which compose the L3 farm receive event fragments from the EVB and build complete events into the appropriate data structure for analysis. Since it takes about one second for one computer unit to make a trigger decision on one event, it takes a large farm of 250 Dual Pentium Linux personal computers (called "processors") to ensure the required input rate. Each subfarm contains between 14 and 18 processor nodes and one "converter" node, which acts as "farm input" distributing the data flow coming from the EVB.

The events are then passed to a trigger algorithm (a different one for each processor) 
that categorizes the event and makes the decision as to whether or not to permanently store it. The selected events are passed to the Data Logger subsystem. During the building processing, the event integrity is checked. The L3 algorithms take advantage of the full detector information and improved resolution unavailable to the lower trigger levels. This includes full three-dimensional track reconstruction and tight matching of tracks to calorimeter and muon-system information. Results from the lower levels are used to drive the algorithms, which are based on the off-line analysis packages. This is a modular and separated filter modules for specific triggers. L3 accepts events with a rate of approximately $75 \mathrm{~Hz}$.

\subsubsection{Event Generation and Simulation}

Based on the known physics of the processes under study, we have developed computer programs which

1. reproduce the kinematics of the physics processes, which in the case of the physics of elementary particles involves the production and decay of the examined particles;

2. simulate the effect of the passage through matter (represented by the detector) of these particles, simulating the amount of energy they will lose, the direction they will take and so on.

The first step is called "generation" and it is usually performed by sophisticated computer programs called "Monte Carlo". They assemble the known physics of several processes and reproduce their kinematic characteristics, such as $Z$ production from proton-antiproton interactions. That is, knowing the energy of the incoming proton and antiproton and they will assign a certain energy to the $Z$ according to the Parton Distribution Functions, a certain boost, and so on.

After the generation is performed, these data are passed through the "simulation" program. This reproduces the physics of the interaction of the generated particles through the matter of the detector, their consequent decay, and the amount of energy they deposit in each subdetector. In CDF a GEANT [47] parameterization of the detector is used, which contains all the information regarding the amount of material in the detector and the geometry of its components. The output of the simulation program has exactly the same structure as the actual data taken from real interactions, consisting of a series of hits in the COT or energy depositions in the calorimeters or in the muon chambers. To reproduce the real event coming from a $p \bar{p}$ interaction, many different physics concepts have to be introduced and coded into the simulation program, such as bremsstrahlung, to realistically reproduce the behavior of the particles passing through the detector. Once the simulated data have been processed, they are analyzed by the same analysis code used to analyze the collision data; the agreement of the two 
gives confidence that both physics and the detector response are well understood and under control. Any inconsistencies between the data and the simulation is indicative of lack of knowledge or deficiencies in the code, and thus needs to be investigated.

\subsubsection{Object-oriented Software}

All the reconstruction and data acquisition system at CDF is built upon a $\mathrm{C}++$ infrastructure. $\mathrm{C}++$ is an object-oriented software language which makes use of "object", intended as separate self-existing structures of a general nature identifying all entities of the same kind. An object defining a certain category will contain by definition all the properties and links to the quantities common to that particular category. In CDF these objects are the most basic physics quantities such as a track, or the more sophisticated ones such as electrons, muons, and jets. According to this philosophy, a separate piece of software exists to identify each separate physics object. Once the basic requirements to form a physics object are fulfilled, the latter can be filled with links to all the physics properties or quantities which can help to better identify it. As an example, a "jet object" will contain a certain value for the energy measured in the hadronic calorimeter, one for the EM energy, the coordinates of its position in the detector, and so on. In general, for each object only one quantity of kind is chosen to be associated with it. In this way the user who has access to the object will obtain the energy or the pseudorapidity value which has been uniquely associated to it by selection criteria defined "a priori". 


\section{Chapter 3}

\section{Base-Line Selection of Like-Sign Dilepton Events}

Our physics motivation is to search for the neutral Higgs boson using events containing a like-sign (LS) dilepton pair as expected in the following reaction:

$$
q q^{\prime} \rightarrow W^{ \pm} H \rightarrow W^{ \pm} W^{(*)} W^{(*)} \rightarrow \ell^{ \pm} \ell^{ \pm}+X
$$

Relevant Higgs boson mass-regions are above $160 \mathrm{GeV} / c^{2}$ for the Standard Model Higgs boson where the branching fraction of $H \rightarrow W W^{*}$ supersedes that of $H \rightarrow b \bar{b}$, and above $110 \mathrm{GeV}$ for the bosophilic (fermiophobic) Higgs boson where the branching fraction of $H \rightarrow \gamma \gamma$ is overtaken by this channel. In this chapter we summarize the physics objects at CDF experiment and datasets which we use first. We then describe the base-line event selection for a like-sign dilepton $(e e, e \mu$, and $\mu \mu)$ sample that we use in our Higgs boson search analysis. The sample is extracted from inclusive high- $p_{T}$ lepton datasets collected by central electron and muon triggers. The corresponding integrated luminosity is about $200 \mathrm{pb}^{-1}$. Our base kinematical-cut is $E_{T}\left(p_{T}\right)>20$ $\mathrm{GeV}(\mathrm{GeV} / c)$ for the leading electron (muon) and $6 \mathrm{GeV}(\mathrm{GeV} / c)$ for the 2nd leading electron (muon). The selection also includes isolation cuts, lepton identification cuts that are fairly "standard", and minimal requirements on lepton pairs. We observed 45 events after the selection.

\subsection{Physics Objects at CDF}

\subsubsection{Track}

The helical track is described by five parameters: impact parameter $\left(d_{0}\right)$, curvature $(C)$, azimuthal angle $\left(\phi_{0}\right), z_{0}$, and $\lambda=\cot \theta_{0}$ [48]. There are illustrated in Figure 3.1. As explained in the previous chapter, charged particles leaves small energy de- 

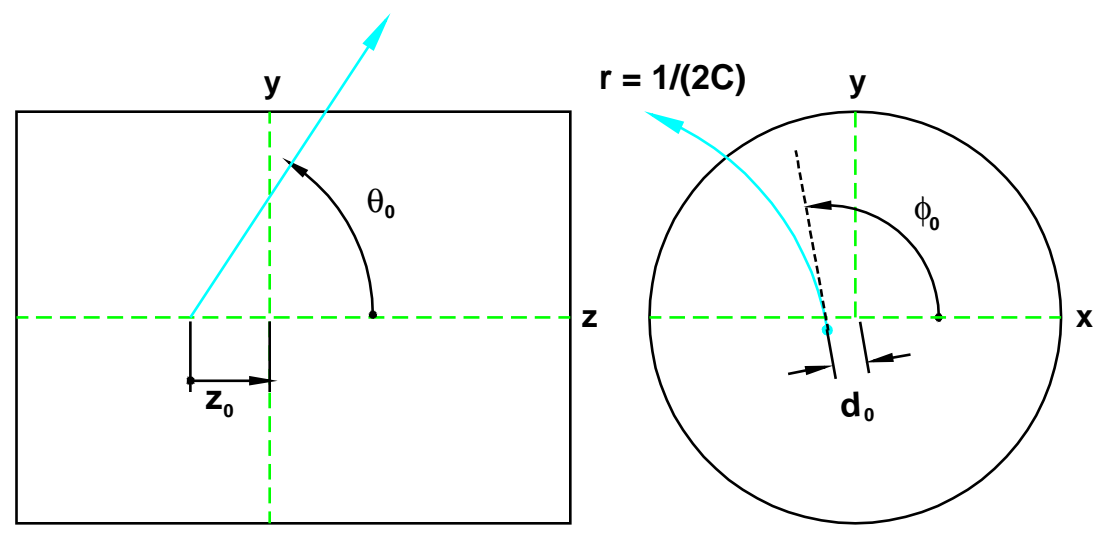

Figure 3.1: An illustration of the tracking parameters: the figure on the left shows a charged track in the $r-z$ view of the tracking volume. The figure on the right shows an $r-\phi$ view of the track. $d_{0}$ is the track's impact parameter, or point of closest approach to the origin. $C$ is the curvature.

positions when they pass through the tracking system. By following "tracking" or "pattern recognition" algorithms we can reconstruct the charged particle track. There are several pattern recognition algorithms used to reconstruct tracks in the CDF tracking system. Most of the tracks are reconstructed using "Outside-In" algorithms which we will describe here. The name of this group of algorithms suggest that the track is followed from the outside of the tracking system to inward.

When projected into the two dimensional $r-\phi$ plane, the helical track is a circle. This simplifies pattern recognition, so the first step of pattern recognition in the COT looks for circular paths in radial superlayers of the COT. Supercells in the radial superlayers are searched for sets of four or more hits that can be fit to a straight line. These sets are called "segments". The straight-line fit for a segment gives sufficient information to extrapolate rough measurements of curvature $C$ and $\phi_{0}$. Once segments are found, there are two approaches to track finding. One approach is to link together segments for which the measurements of curvature $C$ and $\phi_{0}$ are consistent. The other approach is to improve the curvature $C$ and $\phi_{0}$ measurement of a segment reconstructed in superlayer eight by constraining its circular fit to the beamline, and then adding hits which are consistent with this path. Once a circular path is found in the $r-\phi$ plane, segments and hits in the stereo superlayers are added by their proximity to the circular fit. This results in a three-dimensional track fit. Typically, if one algorithm fails to reconstruct a track, the other algorithm will not. This results in a high track reconstruction efficiency (about 95\%) in the COT tracks which pass through all eight superlayers $\left(p_{T}>400 \mathrm{MeV} / c\right)$. The track reconstruction efficiency mostly depends on how many tracks there are to be reconstructed in the event. If there are many tracks 

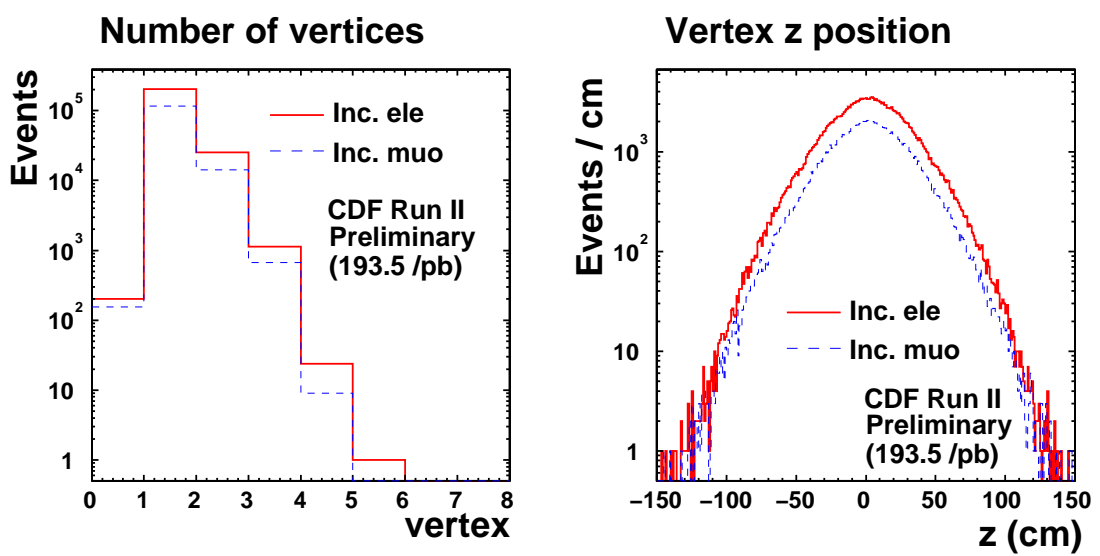

Figure 3.2: Left distributions are number of vertices in inclusive high $p_{T}$ lepton samples. Right one are primary vertex z position. Solid lines are electron sample and broken ones are muon sample. We require at least one tight lepton for each event.

present close to each other, hits from one track are shadowed by hits from the other track and resulted in efficiency loss. Once a track is reconstructed in the COT, it is extrapolated into the Silicon tracking system. Based on the estimated errors on the track parameters, a three-dimensional "road" is formed around the extrapolated track. Starting from the outermost layer, and working inward, silicon clusters found inside the road are added to the track. As a cluster gets added, the road gets narrowed according to the knowledge of the updated track parameters. Reducing the width of the road reduces the chance of adding a wrong hit to the track, and also reduces computation time. In the first pass of this algorithm, $r-\phi$ clusters are added. In the second pass, clusters with stereo information are added to the track.

\subsubsection{Vertex}

In this analysis it is important to require that the two leptons are coming from the same event primary vertex point. The event primary vertex is reconstructed from tracks. In the vertex finding algorithm a track is applied cuts to check the quality of the track first. At least two stereo and two axial superlayers with at least six hits each have to be assigned to a COT track to accept this track. Up to now no cuts are performed on the hit content of silicon tracks. The main task of the algorithm is to find the tracks that originated from the primary vertex and remove all other tracks from the vertex fit. Using a wrong track in the fit results in getting a primary vertex position and covariance matrix that is not compatible with the right vertex position. To remove the tracks that do not originate from the primary vertex the fitting procedure is iterative. It starts with fitting a vertex with all tracks that passed the track selection cuts. Then 
it loops over all the tracks and subtracts one track a time from the fit and calculates the $\chi^{2}$ of this track with respect to the fitted vertex. If the highest $\chi^{2}$ value for any of the tracks exceeds a specified value, this track is removed from the track sample. Then all remaining tracks are used to fit a vertex and this pruning procedure is repeated. If all tracks pass the $\chi^{2}$ cut, the tracks go through the same procedure again doing a vertex fit with tweaking of the track parameters this time. This pruning of the track collection stops if a specified minimum number of tracks is left or all tracks pass the $\chi^{2}$ cut. A last vertex fit is done with the remaining tracks to find the primary vertex position. For example, Figure 3.2 shows the number of vertices and primary vertex $\mathrm{z}$ position. We require at least one "tight" lepton for each event. The "tight" lepton is described in section 3.2.2.

\subsubsection{Electron}

\section{Electron Clustering}

The creation of an electron object begins in the calorimeter. The search for an electromagnetic (EM) shower in the calorimeter begins with all the towers which have more than $3 \mathrm{GeV}$ of EM transverse energy. These are defined as seed towers. The list of seed towers is ordered in transverse energy from the highest to the lowest. The towers adjacent in $\eta$ to the highest $E_{T}$ seed tower ("shoulder towers") form a "cluster" in the central electromagnetic calorimeter (CEM) together with the seed tower itself if they have at least $100 \mathrm{MeV}$ of EM or hadronic energy. The shoulder towers have to be within the same wedge in $\phi$ as the seed tower; this means that the cluster has to be contained in a single wedge and can consist of, at most, the seed tower and its nearest neighbors in $\eta$ on either side of it. The maximum cluster size is 3 towers in $\eta$ and 15 degrees in $\phi$. If the seed tower is either in the outer or in the innermost region of the CEM (so called "tower 0" and "tower 9"), then only "tower 1" or "tower 8" is added to the cluster, and no towers of a cluster are allowed to cross a region boundary or the center of the detector. Only electromagnetic energy is used to determine the centroid and the total energy of the cluster.

\section{Creation of Electron Object}

When a valid EM cluster is found, it is added a series of other objects e.g. tracks, a shower-max cluster, and a pre-radiator cluster, then added to the "CdfEmObject" to form an electron. For each CdfEmObject, each track is in turn iteratively extrapolated to the plane of the CES for the wedge containing the associated EM cluster. The final extrapolated track is required to be within $25 \mathrm{~cm}$ in $x$ (where $x$ is the local $x$ from the CES) and $38 \mathrm{~cm}$ in $z$ from the center of the EM tower seeding the cluster. This provides a region in the CEM that covers slightly more than three physical towers. Among the tracks which fulfill these requirements, the "best matching" one is chosen to be the highest $p_{T}$ track located within the seed tower or not more than 5 
$\mathrm{cm}$ beyond the seed tower boundary in the $z$ direction, which passes some "Quality Cuts" requiring a minimum number of COT axial and stereo hits. The next objects checked for association with the CdfEmObject are shower-max clusters. In the central calorimeter there are two CES cluster collections; an "unbiased" collection which is created from a list of wires or strip seeds over a threshold energy (150 MeV), typically used in the creation of photon objects, and a "track based" collection that uses the wire or strip nearest an extrapolated track as a seed; the latter is used in electron analysis. The best matching one is taken to be the one with the highest energy. For the "track based" CES clusters, the track seeding each CES cluster is required to belong to the collection of tracks which have been found to match the CdfEmObject. Among these CES clusters, the "best matching" one is chosen to be the one seeded by the best track previously determined. This is the CES cluster which will be used in this analysis.

\section{Correction of Electron Object}

In this section the corrections applied to the central electron variables in the data are introduced.

\section{(a) Vertex Correction}

The electron transverse energy in CdfEmObject is calculated assuming that the interaction point is located at $z=0$. We recalculate the transverse energy $E_{T}$ using $z_{0}$ of the track [49] associated to the electron as the event interaction point, and the angle of the track as the direction of the electron.

\section{(d) Energy Corrections}

In order to tune the central electromagnetic calorimeter, the CEM response needs to be determined and corrected. This procedure includes individual tower gain, local $\mathrm{x}$ and $\mathrm{z}$ position dependent corrections, and attenuation of the light passing through the scintillator.

- Correction for tower-to-tower gain:

The corrections for the tower-to-tower gain variations have been determined using a calibration electron sample collected during the first stage of Run II data-taking. The gains are defined as the average $E / p$ for each tower in the window $0.8-1.25$; their distribution can be observed in Figure 3.3 [50]. Correcting CEM energies for this effect results in approximately $5 \%$ improvement in the energy resolution.

- Correction for the time-dependent

CEM gain changes with time. These time-dependent corrections, extracted by plotting $E / p$ as a function of time, have been implemented in the data. This includes the overall scale correction to make the peak of the dielectron invariant mass be at $91 \mathrm{GeV} / c^{2}$. Figure 3.4 demonstrates that the corrections are made properly. 


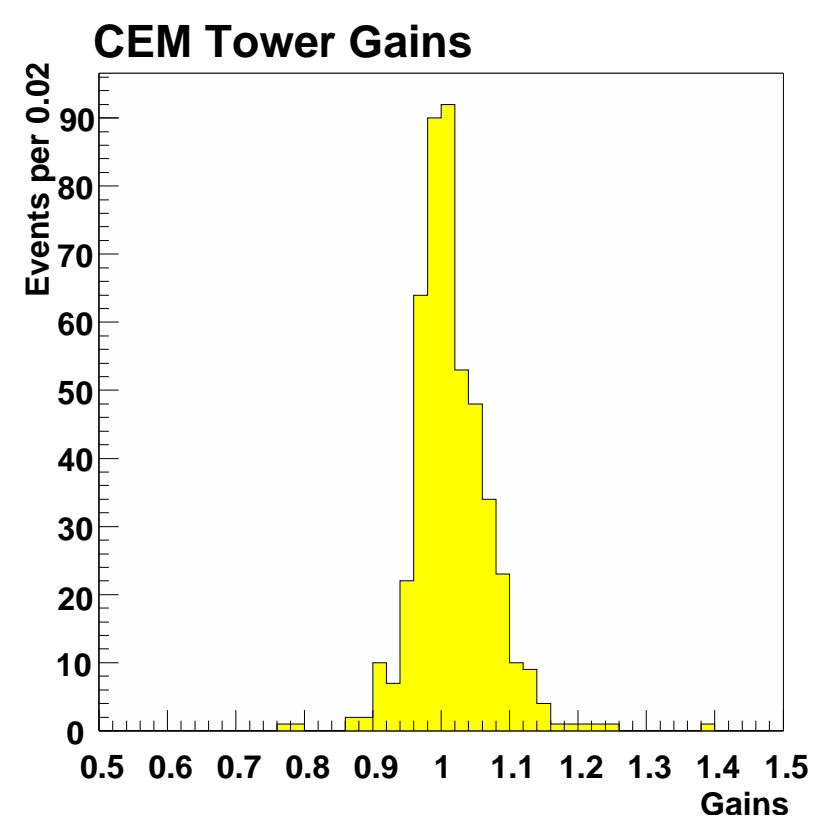

Figure 3.3: CEM tower gains. A global correction factor of 1.015 is applied in order to move the mean back to what it was before the corrections. This prevents these corrections from changing the absolute energy scale.

- CEM face correction:

The CEM response is dependent on local $x$ and $z$ coordinates within a tower. These corrections, called "face corrections", are extracted from the test-beam data taken in 1994 and applied [51] to both data and simulation. Corrections for the attenuation of light passing through the scintillator toward wavelength shifters and other corrections are also applied at this stage.

Despite these corrections, about $7 \%$ variation was observed when $E / p$ was plotted as a function of the CES local $x$ position. In order to make a flat distribution of $E / p$ versus CES local $x$, the following correction factor is applied to CEM energy:

$$
f_{x}=\frac{1.015}{\left(1+0.000157 \times x^{2}\right)} .
$$

where $x$ is measured by the CES. The corrected CEM energy is $E_{\text {corr }}=f_{x} \times E$. The correction factor and $E / p$ as a function of CES local $x$ after that the corrections are applied are shown in Figure 3.5.

\section{(c) Beam Constraint}

Tracking resolution tends to dominate the $E / p$ resolution once the energy has been 


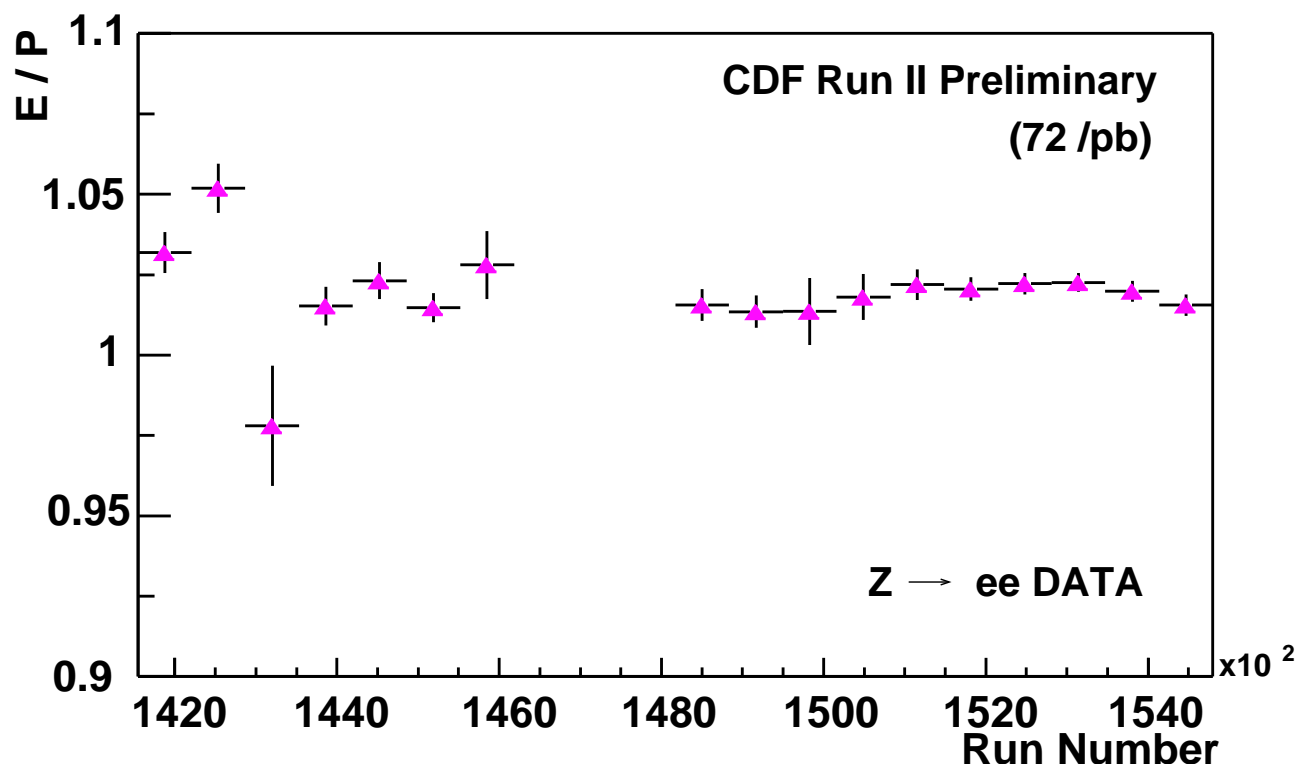

Figure 3.4: $E / p$ as a function of run number. The average $E / p$ is calculated in the range between 0.9 and 1.1 .

tuned. The raw COT resolution can be substantially improved by imposing a beam constraint [52], that is forcing the track fit to go through the beam-split ( $x$ and $y$ position only). All the tracks used in this document are beam constrained COT tracks.

\section{Central Electron Variables}

The variables used in the selection of the central electrons are summarized in the following paragraphs.

- $z_{\mathrm{v} t x}$ :

This variable is the $z$ coordinate of the interaction vertex where the electron has originated. The $z$ position of the primary vertex is used in this context (Figure $3.2)$.

- Fiduciality:

This variable ensures that the electron is reconstructed in a region of the detector which is well instrumented. The electron position in the CEM is determined using either the value determined by the CES shower ("unbiased") or by the extrapolated track ("track based"), and it must satisfy the following requirements:

- the electron must lie within $21 \mathrm{~cm}$ of the tower center in the $r-\phi$ view in order for the shower to be fully contained in the active region; this corre- 

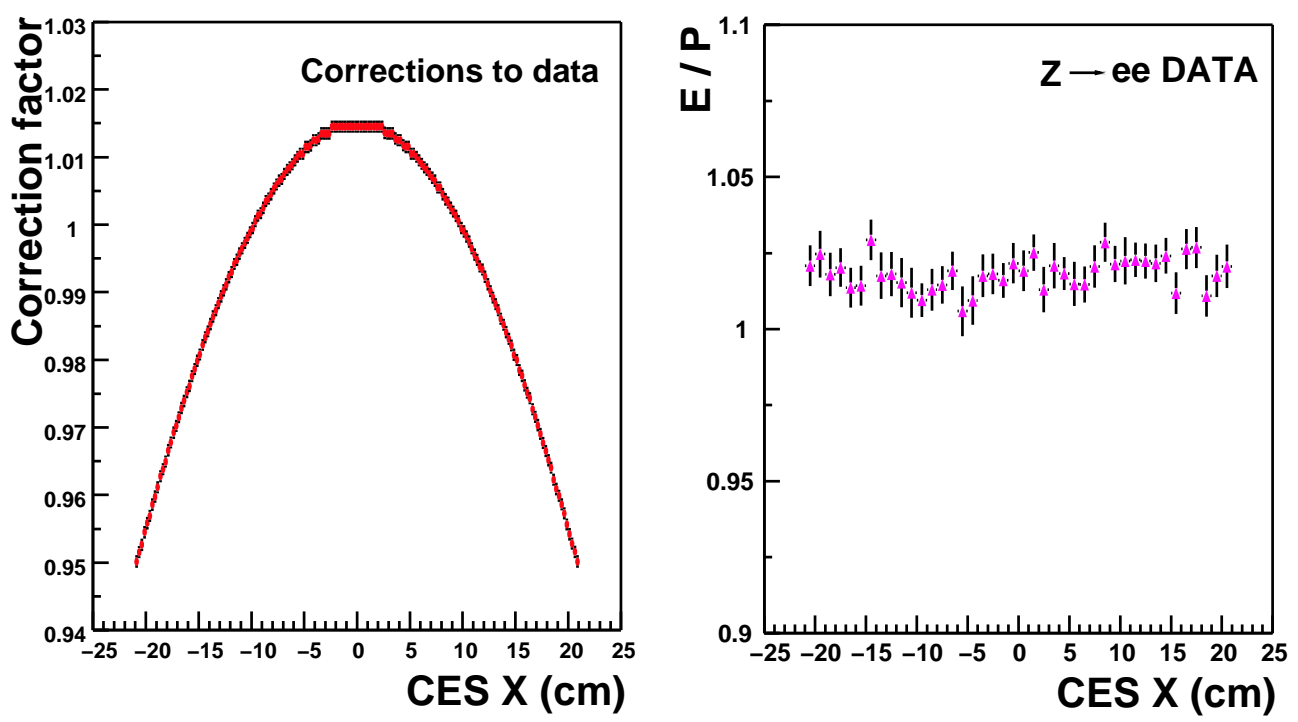

Figure 3.5: Left plot is the CEM energy correction factor applied to the data. Right one is $E / p$ as a function of CES $x$ after the corrections.

sponds to the cut $\left|x_{C E S}\right|<21 \mathrm{~cm}$, where $x_{C E S}$ is the local coordinate of the calorimeter tower,

- the electron should not be in the regions $\left|z_{C E S}\right|<9 \mathrm{~cm}$, where the two halves of the central calorimeter meet, and $\left|z_{C E S}\right|>230 \mathrm{~cm}$, which corresponds to outer half of the last CEM tower ("tower 9"). This region is prone to leakage into the hadronic part of the calorimeter,

- the electron should not be in the region immediately closest to the point of penetration of the cryogenic connections to the solenoidal magnet (the "chimney"), which is un-instrumented. This corresponds to $0.77<\eta<1.0$, $75<\phi<90$ degree, and $\left|z_{C E S}\right|<193 \mathrm{~cm}$.

In addition, the region $1.05<|\eta|<1.10$ is excluded because of the smaller depth of the electromagnetic calorimeter.

- CdfEmObject cluster $E_{T}$ :

An electron cluster is formed from a seed EM tower and a number of shoulder EM towers, which are added to the seed tower until the maximum cluster size is reached. This is defined by three towers in pseudorapidity $(\Delta \eta \sim 0.3)$ and one tower in azimuth $\left(\Delta \phi \sim 15\right.$ degrees). The transverse energy $E_{T}$ is calculated as the EM cluster energy times $\sin \theta$, where $\theta$ is calculated from event vertex point. 
The $E_{T}$ distribution from $Z \rightarrow e^{+} e^{-}$candidates events is shown in Figure 3.6.

$$
E_{T}=E \times \sin \theta
$$

- $p_{T}$ :

This variable is the transverse momentum $\left(p_{T}=p \times \sin \theta\right)$ of a track in the COT (Figure 3.6). The track is selected as the beam-constrained COT track with the highest momentum pointing to the electron cluster.

- Isolation:

This variable is defined by the following:

$$
\begin{aligned}
& E_{T}^{\text {clust }}=E_{T}^{\text {seed }}+E_{T}^{(\eta+1)}+E_{T}^{(\eta-1)}, \\
& I S O_{0.4}^{\text {cal }}=\left(\sum_{\Delta R<0.4} E_{T}^{\text {cal }}\right)-E_{T}^{\text {clust }} .
\end{aligned}
$$

where $E_{T}^{\text {clust }}$ is cluster $E_{T}$ as described before and $\Delta R\left(=\sqrt{\Delta \eta^{2}+\Delta \phi^{2}}\right)$ is defined between the cluster centroid and the center of a candidate tower (Figure 3.7).

- $H A D / E M$ :

This variable is the ratio of the total energy in the hadron calorimeter to the total energy in the EM calorimeter for the towers includes in the EM cluster (Figure 3.7). This cut value has a flat efficiency up to $175 \mathrm{GeV}$ as observed in test beam data $[63]$.

- $L_{\mathrm{shr}}$ :

The purpose of this quantity is to provide some discrimination of electrons and photons from hadronic showers faking these particles in the central electromagnetic calorimeter. This is done by comparing the observed sharing deposition between towers in the CEM to that expected for a "true" electromagnetic shower, taken with test-beam data and recorded in the database. The $L_{\text {shr }}$ (Lateral Shower Sharing variable, shown in Figure 3.7) represents the amount of lateral sharing and it is defined as

$$
L_{\mathrm{shr}}=0.14 \sum_{i} \frac{E_{i}^{a d j}-E_{i}^{\exp }}{\sqrt{(0.14 \sqrt{E})^{2}+\left(\Delta E_{i}^{e x p}\right)^{2}}},
$$

where the sum is only over towers in the electron cluster, not extending across region boundaries or $\eta=0$, and

- $E_{i}^{a d j}$ is the measured energy in tower adjacent to the seed tower, 
- $E_{i}^{e x p}$ is the expected energy in the adjacent tower, calculated using a parameterization from test beam data,

- $\sqrt{0.14 \sqrt{E}}$ is the error on the energy measurement, and

$-\Delta E_{i}^{e x p}$ is the error on the energy estimate.

- $E / p$ :

This quantity is defined by the ratio of the cluster energy $E$ to the momentum of the track (Figure 3.7). The track is selected as the beam constrained COT track with the highest momentum pointing to the electron cluster. During the passage through the material up to the COT active volume the electron might radiate a photon (external bremsstrahlung), which is collinear with the electron and generally deposits energy in the same calorimeter cell as the electron, thus not much affecting the value of $E_{T}$.

- $\chi_{\text {strip }}^{2}$ and $\chi_{\text {wire }}^{2}$ :

The pulse height shape in the Central Electromagnetic Shower-Max (CES) detector is compared to the one obtained with test-beam data using a $\chi^{2}$ test (Figure 3.8). The variable $\chi_{\text {strip }}^{2}$ is the $\chi^{2}$ of the fit between the energy deposited on each of the 11 strips in $z$ in the CES shower and the shape obtained using test beam data. An energy dependent correction is used in the calculation of the $\chi^{2}$, where the total energy of the cluster is the scale factor. A similar variable $\chi_{\text {wire }}^{2}$ tests the energy deposition on the wires in the $r-\phi$ view. The latter is not used in the selection of the events as it is largely affected by bremsstrahlung emission.

- $\Delta x_{\mathrm{CES}}$ and $\Delta z_{\mathrm{CES}}$ :

These variables are the differences between the $x$ and $z$ coordinates of the track extrapolated to the CES and the value of $x$ and $z$ as measured by the CES itself. $\Delta x$ is the separation in the $r-\phi$ view, while $\Delta z$ is the separation in the $z$ view (Figure 3.8). The cut on $\Delta x$ has been multiplied by the sign of the charge of the electron and it is asymmetric in $r-\phi$, to account for photons emitted in bremsstrahlung radiation, which distort the CES cluster toward one direction depending on the charge. Both quantities are corrected for the CES and COT alignments wedge-by-wedge.

- Track Quality:

To ensure that the track associated with the electron is a good quality reconstructed track, we ask that the track has been reconstructed in the COT in 3 axial and 3 stereo superlayers with at least 7 hits in each.

- Separation cut between track and primary vertex $z$ position, $\left|z_{0}-z_{\mathrm{vtx}}\right|$ :

$z_{0}$ parameter is recalculated to take the $\mathrm{z}$ coordinated of the closest-approach point with respect to run-average beam line (Figure 3.8). 
- Impact parameter, $d_{0}$ :

The impact parameter $d_{0}$ is recalculated to take the $\mathrm{z}$ coordinated of the primary vertex (Figure 3.8).

- Conversion removal:

Two variables for the conversion pair candidate tagging are used:

$$
|\Delta(\cot \theta)|<0.04, \text { and }\left|\delta_{x y}\right|<0.2 \text {. }
$$

The variable $\Delta(\cot \theta)<0.04$ is simply the difference in $\cot \theta$ of the two tracks. The $x-y$ separation $\delta_{x y}$ is found by first collapsing the helices of the two tracks into two circles on the $x-y$ plane. Two variables, $\Delta(\cot \theta)$ and $\delta_{x y}$, of the typical conversion in data $\left(E_{T}>4 \mathrm{GeV}\right.$ conversion sample) and illustrations are shown in Figure 3.9. 

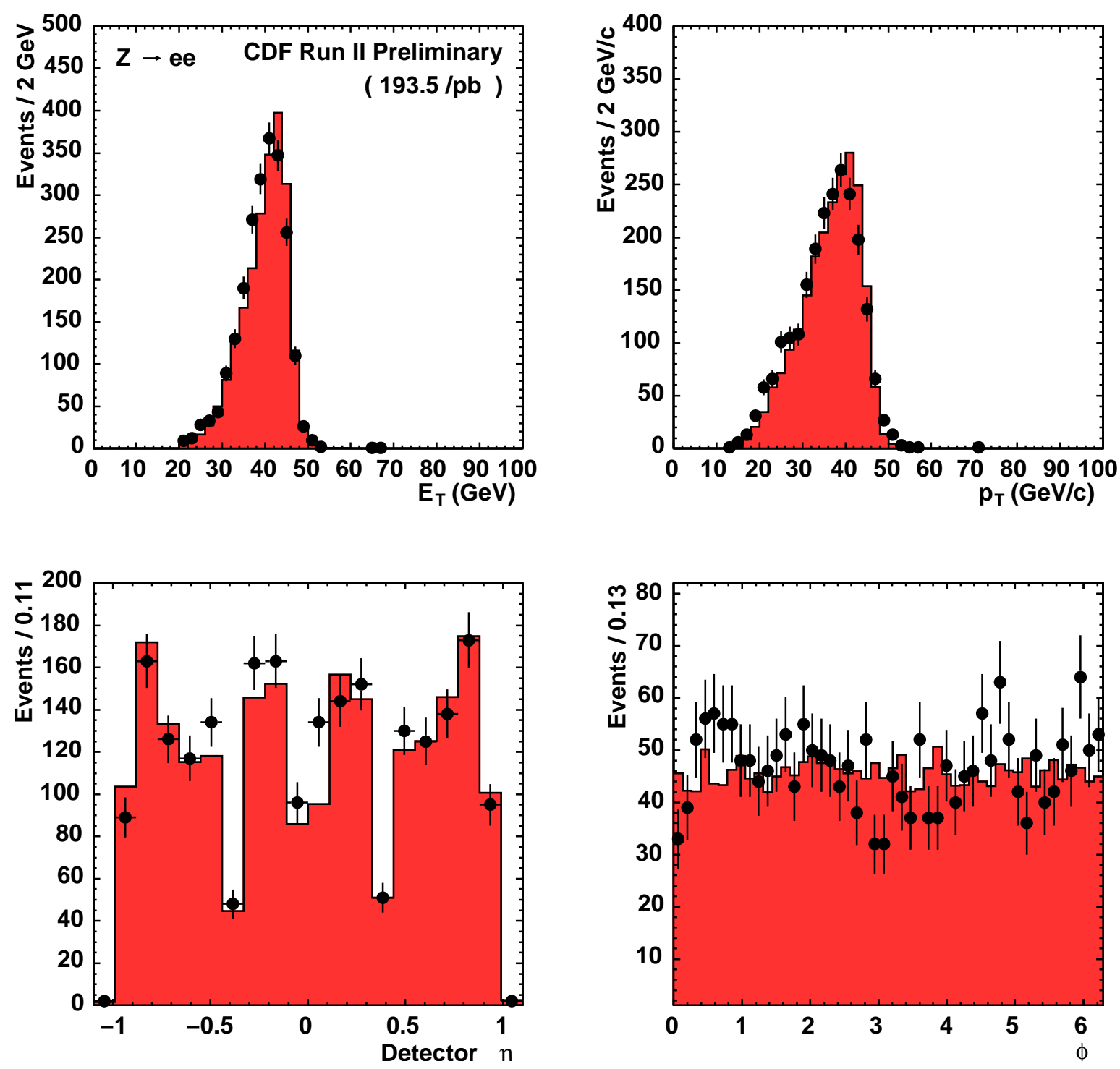

Figure 3.6: The electron variables used for the selection of the events. The tight electron in the $Z \rightarrow e^{+} e^{-}$candidates events (dots) and in the Monte Carlo (solid histogram) are used. For each variable all the selection criteria, but the one including the variable itself, are applied. The number of Monte Carlo events is normalized to the $193.5 \mathrm{fb}^{-1}$ data. Upper plots are electron $E_{T}$ and $p_{T}$ distributions and lower ones are detector $\eta$ and azimuthal angle $\phi$ of EM cluster from $Z \rightarrow e^{+} e^{-}$candidate events. 

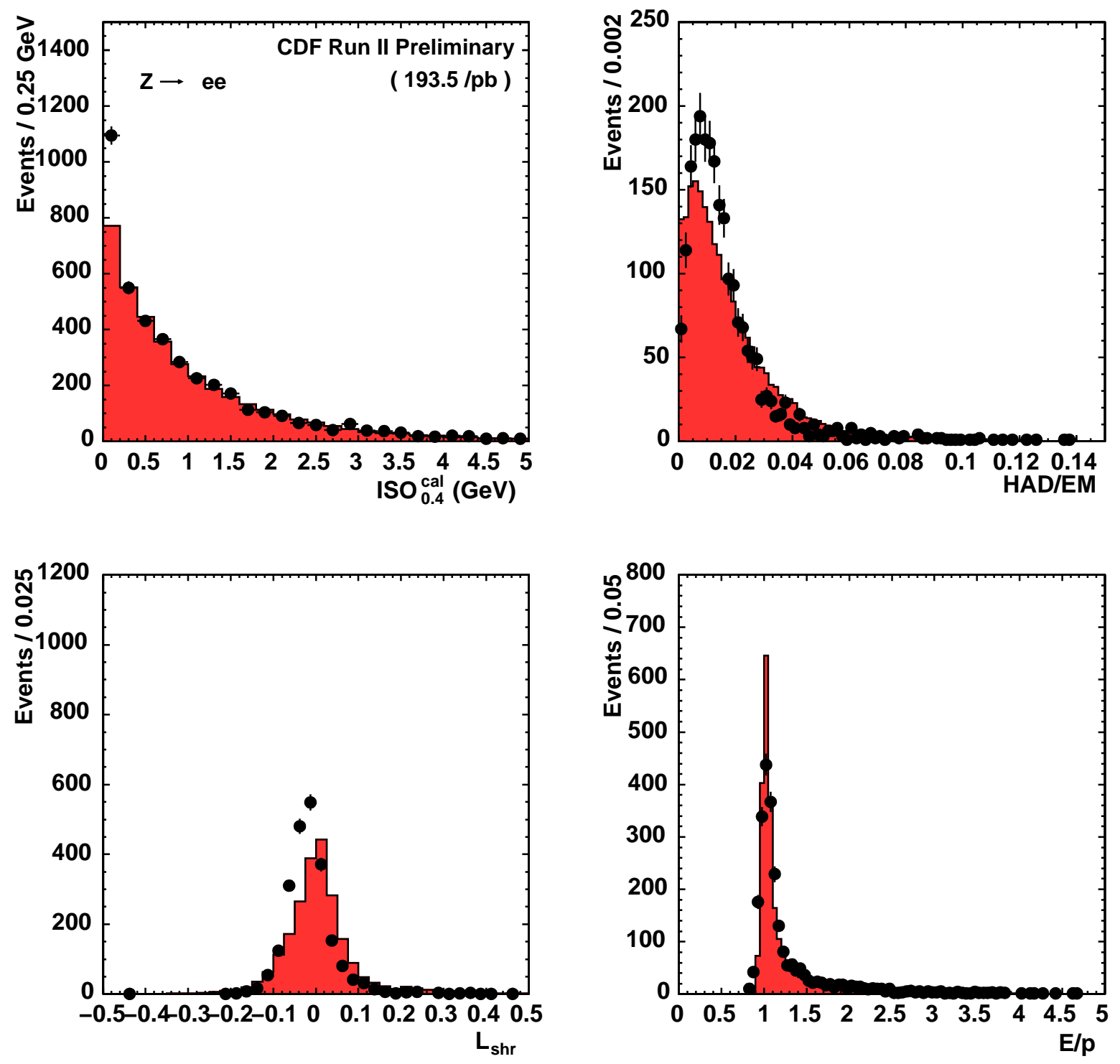

Figure 3.7: The electron variables used for the selection of the events. The tight electron in the $Z \rightarrow e^{+} e^{-}$candidates events (dots) and in the Monte Carlo (solid histogram) are used. For each variable all the selection criteria, but the one including the variable itself, are applied. The number of Monte Carlo events is normalized to the $193.5 \mathrm{fb}^{-1}$ data. Upper plots are calorimeter isolation $I S O_{0.4}^{\text {cal }}$ and $H A D / E M$ distributions. Lower plots are $L_{\mathrm{shr}}$ and $E / p$ distributions 

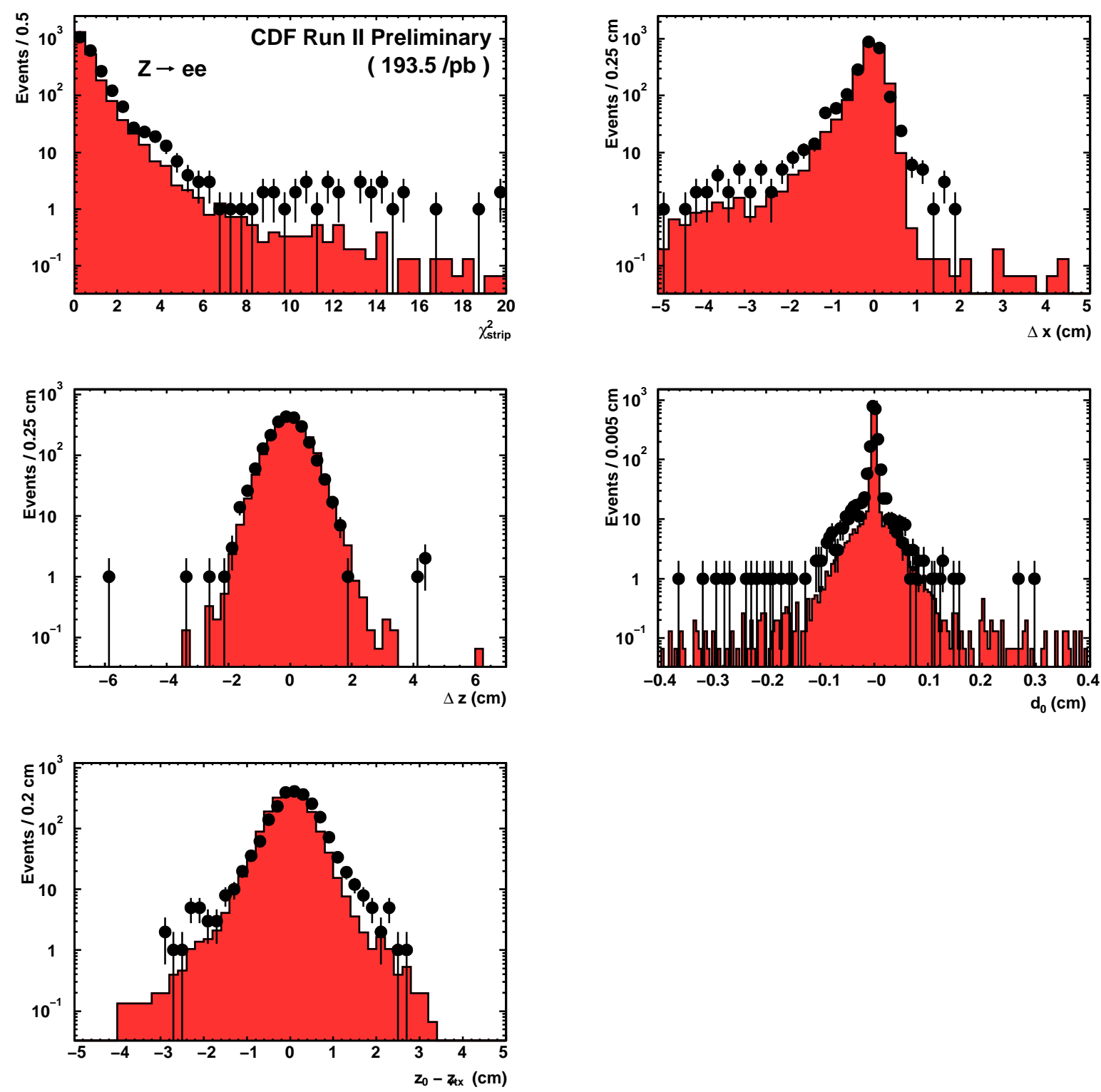

Figure 3.8: The electron variables used for the selection of the events. The tight electron in the $Z \rightarrow e^{+} e^{-}$candidates events (dots) and in the Monte Carlo (solid histogram) are used. For each variable all the selection criteria, but the one including the variable itself, are applied. The number of Monte Carlo events is normalized to the $193.5 \mathrm{fb}^{-1}$ data. Upper plots are CES strip $\chi^{2}$ and $\Delta x \times Q$ distributions. Middle plots are $\Delta z$ and impact parameter $d_{0}$ distributions. Lower plot is $z_{0}-z_{v t x}$ distribution. 

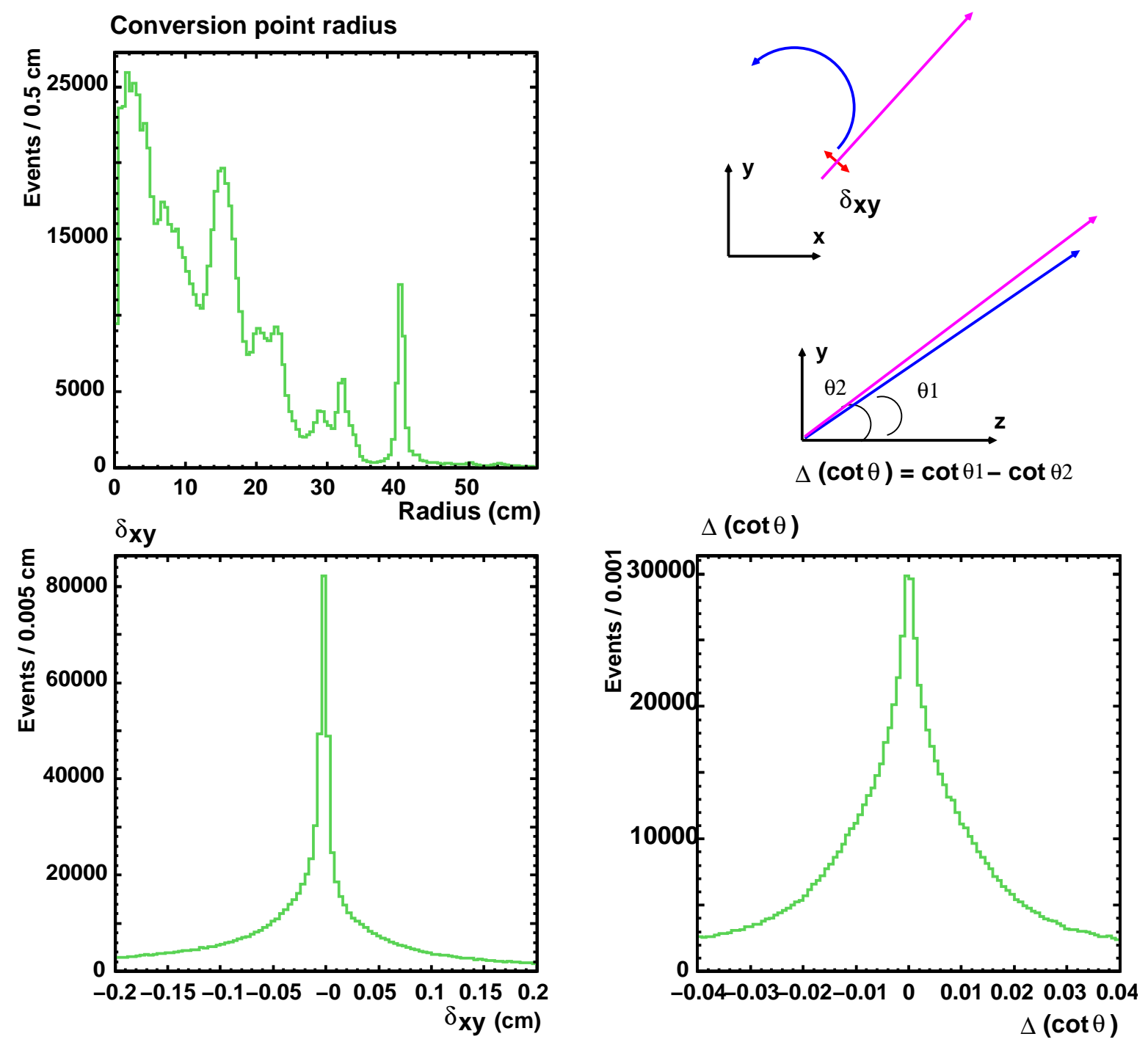

Figure 3.9: Upper plot is a distribution of the conversion point radius from detector center. Lower plots are $\delta_{x y}$ and $\Delta(\cot \theta)$ distributions. These conversions are selected from $4 \mathrm{GeV}$ conversion sample by $|\Delta(\cot \theta)|<0.04$, and $\left|\delta_{x y}\right|<0.2$. 


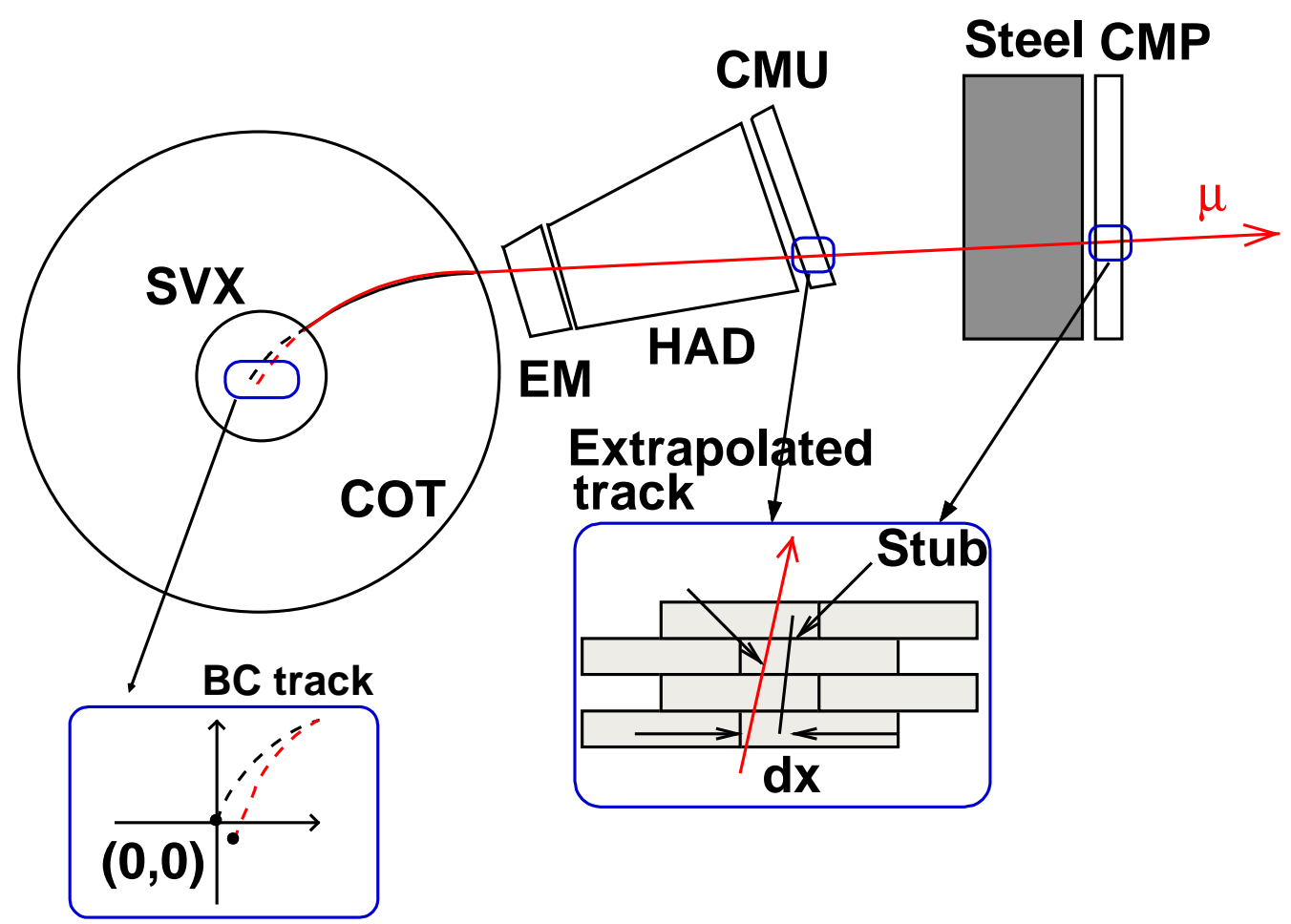

Figure 3.10: Passage of a muon through the CDF detector.

\subsubsection{Muon}

\section{Muon Reconstruction}

The high $p_{T}$ muon data that are collected need to be reconstructed with the CDF offline software with very loose reconstruction requirements and with calibrations and corrections applied. The CDF muon system is very complicated, consisting of many parts with different capabilities. Therefore, we will not go into the details of individual subsystem calibrations here. The common calibrations are the global alignment of the detectors with respect to the central tracker, the drift velocities for a correct measurement of drift distances. The passage of a muon in CDF detector and hitting CMU and CMP chambers is schematically shown in Figure 3.10. The muon track parameters are measured in the tracking volume and the four momentum of a muon comes from its track. The calorimeters, in principle, act like absorbers and they give the measure of the electromagnetic and hadronic energies. The muon then passes through the muon chambers, such that muon chamber tracking (forming "stub") can later on be performed. A muon stub is formed using the hit information and stub finding and fitting algorithms for the sub-detectors. The output of the fits are the stub position and direction vectors [54]. A muon stub has at least three hits associated to it. 
The muon track helices are parameterized at the point of closest approach to the origin in $x-y$ plane of the CDF detector [55]. The axial $r-\phi$ parameters are the impact parameter $d_{0}$, azimuthal angle $\phi_{0}$, and the curvature $C$ or $p_{T}$. The stereo $r-z$ parameters are the $z$ position $z_{0}$ and $\cot \theta_{0}$ or $\theta_{0}$ of the angle with respect to the $z$ axis at the point of origin. The $\cot \theta_{0}$ is defined as $p_{z} / p_{T}$. The curvature is defined as $C=1 / 2 R$ where $R$ is the radius of the curvature of the track as described at the previous track reconstruction section. For a negatively charged particle, the curvature has a negative sign. The relation between $p_{T}$ and curvature is:

$$
p_{T}=\frac{B}{2 c} \times \frac{1}{C}=\frac{0.002117}{C},
$$

where $c$ is the speed of the light. For a tracking volume of fixed magnetic field, $p_{T}$ is only a function of curvature $C$. If the tracks use only the COT information, they are usually "beam-constrained" at the analysis level. This performed refitting the track by using the measured position of the beamline. This procedure improves the momentum resolution of the tracks.

The muon tracking stops at the face of the COT. From then on, a procedure should be applied to match a stub candidate in the muon chambers to the muon track candidate. Also the path of the muon inside the calorimeters is not measured. Therefore, "extrapolators" are used to extrapolate the track to the stub. The measure of this quantity in $r-\phi$ plane, usually called $\Delta x$, is one of the basic criteria for selecting a muon candidate in the CDF detector. Table 3.1 summarizes the criteria for reconstructing a "CdfMuon" in the CDF offline code. The criteria are taken as applied in the version of CDF software used in this analysis. For CMX and BMU detectors, the track-stub matching quantities are applied to the tracks and stubs on the same side of the detector. For CMU this criteria is applied only if $z_{0}$ is more than $20 \mathrm{~cm}$.

\section{Muon Variables}

The variables used in the selection of the central muons are summarized in the following paragraphs.

- Fiduciality:

For the CMUP and CMX muons we require that the CMP or CMX stub satisfies the following two requirements:

- In the direction of the drift wire, the track has to be extrapolated to be at least $3 \mathrm{~cm}$ inside of the chamber: fiducial $z$ distance $<-3 \mathrm{~cm}$ for CMP and CMX,

- In the direction perpendicular to the drift wire, the track has to be extrapolated to be inside of the chamber: fiducial $\mathrm{x}$-distance $<0 \mathrm{~cm}$ for CMP and CMX. 


\begin{tabular}{cc}
\hline \hline Parameter & Criteria \\
\hline$p_{T}$ & $>1.3 \mathrm{GeV} / \mathrm{c}$ \\
& $>10 \mathrm{GeV} / c($ Stub-less muon $)$ \\
$\left|z_{0}\right|$ & $<200 \mathrm{~cm}$ \\
$\left|d_{0}\right|$ & $<6 \mathrm{~cm}$ \\
Axial track hits & $>10$ \\
$\Delta x$ & $<30(\mathrm{CMU}) \mathrm{cm}$ \\
& $<60(\mathrm{CMP}) \mathrm{cm}$ \\
& $<50(\mathrm{CMX}) \mathrm{cm}$ \\
& $<90(\mathrm{BMU}) \mathrm{cm}$ \\
$\Delta z$ & $<250(\mathrm{CMU}) \mathrm{cm}$ \\
& $<330(\mathrm{CMP}) \mathrm{cm}$ \\
& {$[325,550](\mathrm{CMX}) \mathrm{cm}$} \\
& {$[440,840](\mathrm{BMU}) \mathrm{cm}$} \\
\hline \hline
\end{tabular}

Table 3.1: Muon reconstruction criteria.

The convention is that for tracks extrapolated outside the chamber the fiducial distance is greater than zero. For tracks extrapolated inside the chamber the fiducial distance is less than zero. We do not make any Fiduciality requirement on CMU stubs.

- COT exit radius $\rho$ :

CMX muons require that the COT exit radius $\rho$ of the track. $\rho$ can be calculated from the pseudorapidity $\eta$ and $z_{0}$ of the track as follows:

$$
\rho=\frac{\operatorname{sign}(\eta) \cdot z_{\mathrm{COT}}-z_{0}}{\tan \left(\frac{\pi}{2}-\theta\right)}
$$

where a value of $z_{\mathrm{COT}}(=155 \mathrm{~cm})$ is used for the length of the COT. The $\eta$ of the track after the beam constrained refitting should be used. Figure 3.11 is shown the COT exit radius for CMX $W \rightarrow \mu \nu$ candidate events.

- $p_{T}$ :

This variable is the transverse momentum $\left(p_{T}=p \times \sin \theta\right)$ of a track in the COT (Figure 3.12). The track is selected as the beam-constrained COT track with the highest momentum pointing to the electron cluster.

- Calorimeter energy deposition $(E M)$ and $(H A D)$ :

Muons deposit a minimum ionizing signal in the calorimeters (Figure 3.13). Typical calorimeter isolation cuts are:

$$
E M<2 \mathrm{GeV}, \quad H A D<6 \mathrm{GeV} .
$$




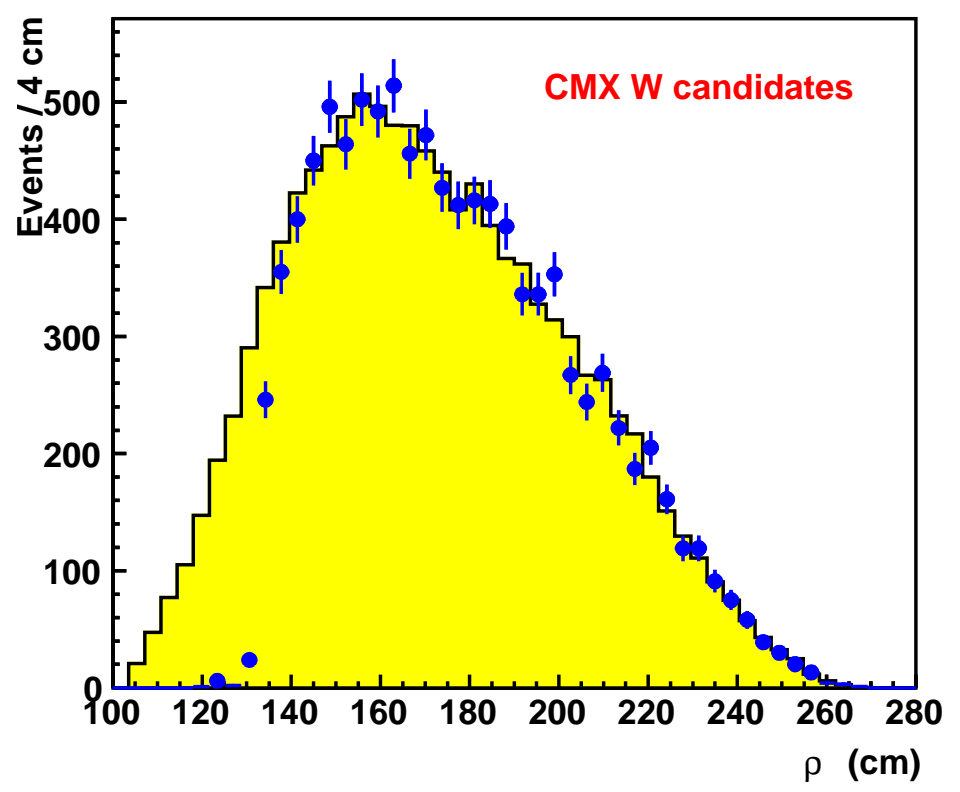

Figure 3.11: The COT exit radius $\rho$ for $\operatorname{CMX} W \rightarrow \mu \nu$ candidate events. The points are the data and the yellow histogram is the simulation. The simulation is normalized to the number of data events in the range $150<\rho<280 \mathrm{~cm}$. The lack of CMX events at low $\rho$ is a reflection of the XFT trigger acceptance due to the requirement of hits on all 4 axial superlayers. A cut of $\rho<140 \mathrm{~cm}$ is chosen to remove this inefficient region.

However, the mean energy deposition in the electromagnetic and hadronic calorimeters increases linearly with momentum and consequently as the muon momenta increase these cuts become less efficient. The mean energy deposition in the hadron calorimeter is given approximately by $2+4 \times p / 500 \mathrm{GeV}$ for momenta greater than $20 \mathrm{GeV} / c$ [60]. The increase originates from large high energy tails from muon interactions such as $e^{+} e^{-}$pair production and bremsstrahlung processes. Muons from $Z$ decay deposit a mean energy $0.4 \mathrm{GeV}$ in the electromagnetic calorimeter and $2.0 \mathrm{GeV}$ in the hadron calorimeter [61], [62]. So although these cuts are suitable for muons for $Z$ decays, they became inefficient for very high momentum muons. For example, a $200 \mathrm{GeV} / c$ muon deposits a mean energy in the hadron calorimeter of $3.6 \mathrm{GeV}$, however, a muon with momentum greater than $500 \mathrm{GeV} / c$ will deposit more than $6 \mathrm{GeV}$ and will consequently exceed above cuts. In order to maintain good efficiency for high energy muons energy dependent cuts are studied [59].

- For $p<100 \mathrm{GeV} / c: E M<2 \mathrm{GeV}, H A D<6 \mathrm{GeV}$,

- For $p>100 \mathrm{GeV} / c$ energy dependent cuts were proposed: 


$$
\begin{aligned}
& E M<2+C_{1} \times(p-100) \\
& H A D<6+C_{2} \times(p-100) .
\end{aligned}
$$

In the analysis this sliding energy cuts are used. $C_{1}$ and $C_{2}$ are chosen to have the values selected in the Run I $Z$ and Drell-Yan Production cross section measurement using dimuons [59]. These values were chosen to maintain the EM energy cut to be $98 \%$ efficient $\left(C_{1}=0.0115\right)$ and the HAD energy cut $97 \%$ effiecient $\left(C_{2}=0.0280\right)$.

- $r \times \phi$ :

These quantities are the track-stub matching in the azimuthal plan $(r-\phi)$ for the central muon detectors (CMU, CMP, and CMX) (Figure 3.14).

- Track Quality:

To ensure that the track associated with the electron is a good quality reconstructed track, we require that the track has been reconstructed in the COT in 3 axial and 3 stereo superlayers with at least 7 hits in each.

- Separation cut between track and primary vertex $z$ position: $\left|z_{0}-z_{\mathrm{vtx}}\right|$ $z_{0}$ parameter is recalculated to take the $\mathrm{z}$ coordinated of the closest-approach point with respect to run-average beam line (Figure 3.14).

- Impact parameter: $d_{0}$

The impact parameter $d_{0}$ is recalculated to take the $\mathrm{z}$ coordinated of the primary vertex (Figure 3.14). 

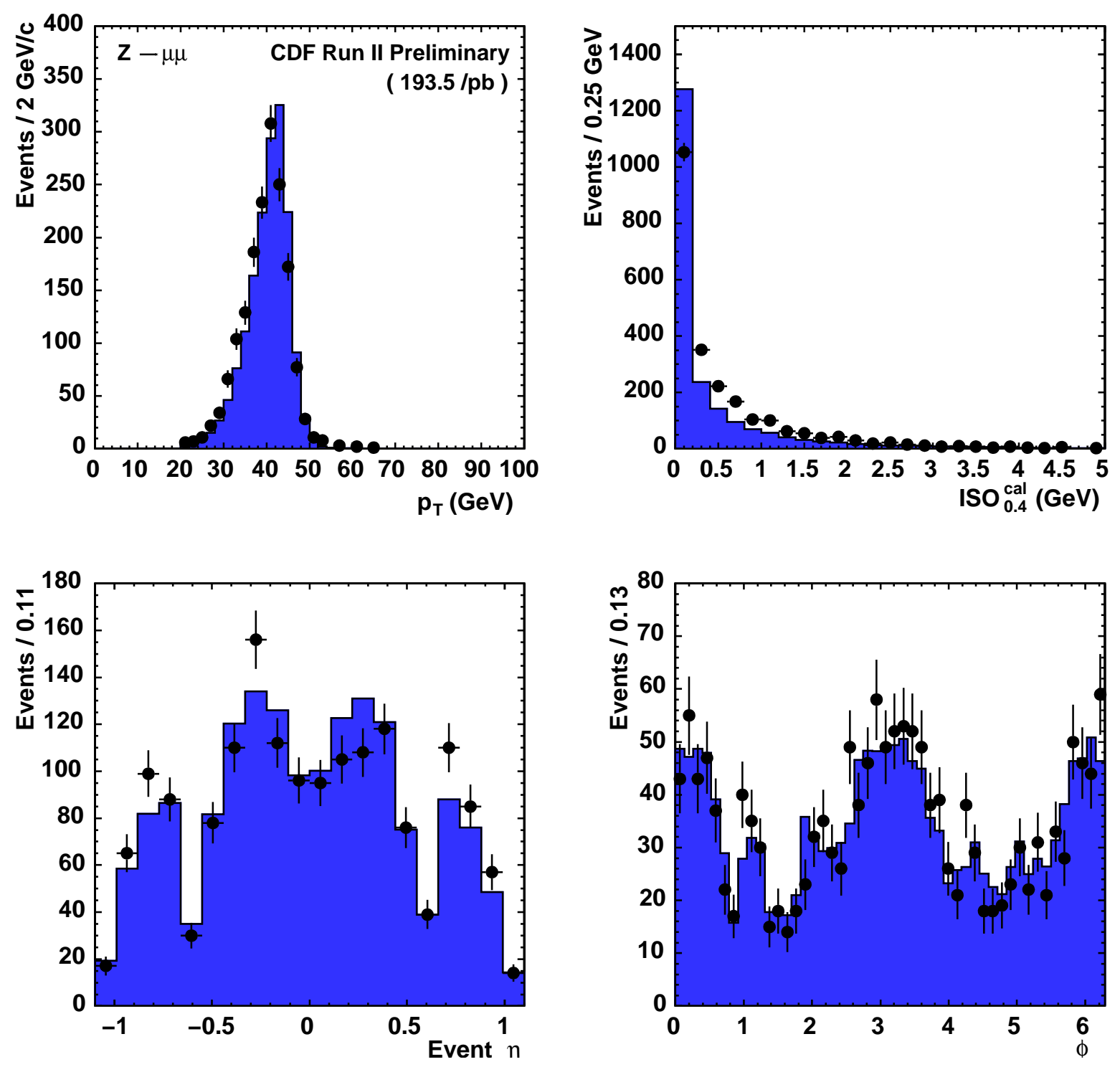

Figure 3.12: The muon variables used for the selection of the events. The tight muon in the $Z \rightarrow \mu^{+} \mu^{-}$candidates events (dots) and in the Monte Carlo (solid histogram) are used. For each variable all the selection criteria, but the one including the variable itself, are applied. The number of Monte Carlo events is normalized to the $193.5 \mathrm{fb}^{-1}$ data. Upper plots are CMUP and CMX muon's $p_{T}$ and $I S O_{0.4}^{\text {cal }}$ distributions and lower ones are event $\eta$ and azimuthal angle $\phi$ of track. 

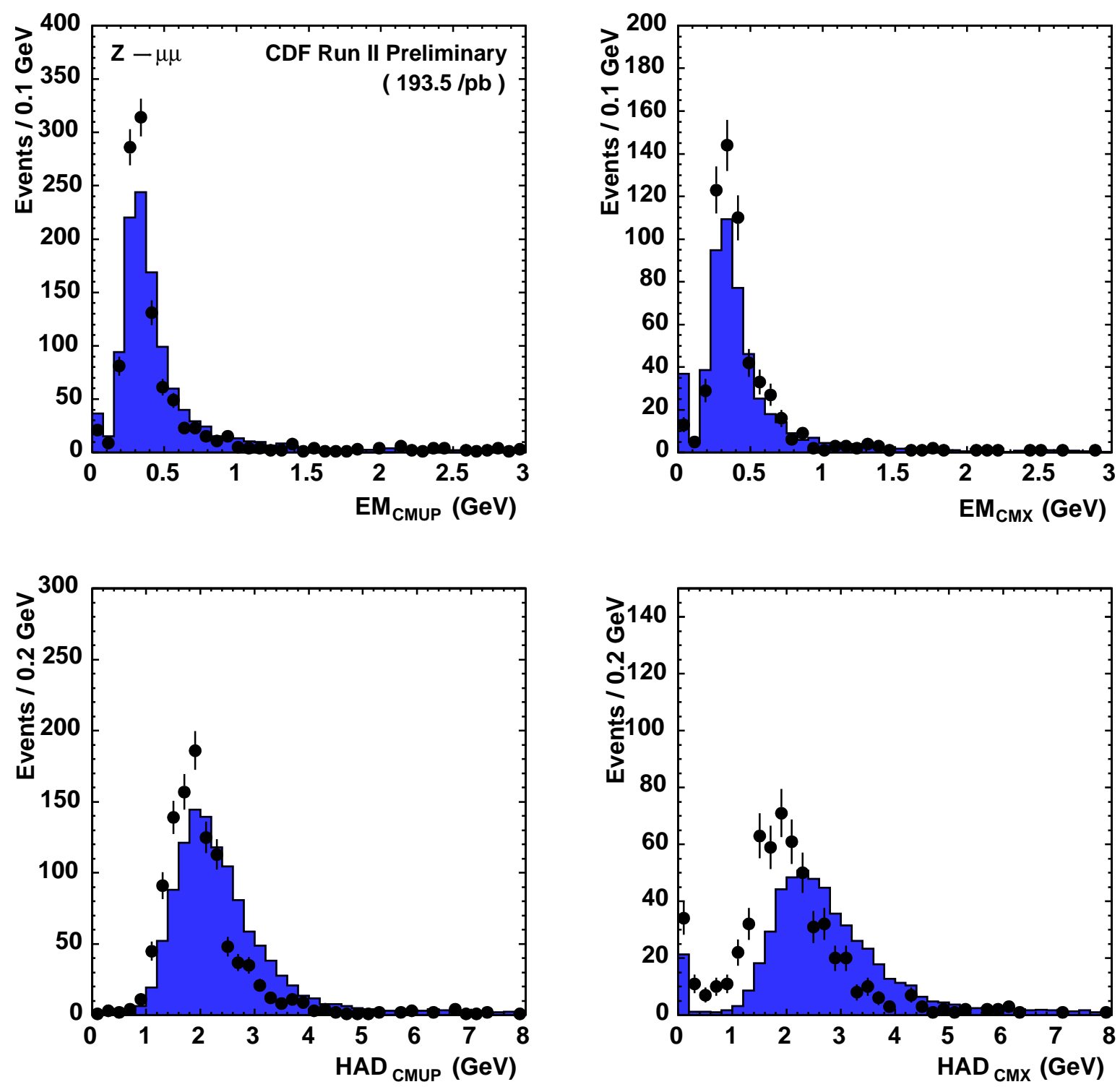

Figure 3.13: The muon variables used for the selection of the events. The tight muon in the $Z \rightarrow \mu^{+} \mu^{-}$candidates events (dots) and in the Monte Carlo (solid histogram) are used. For each variable all the selection criteria, but the one including the variable itself, are applied. The number of Monte Carlo events is normalized to the $193.5 \mathrm{fb}^{-1}$ data. Upper plots are muon electromagnetic calorimeter energy deposition distributions for CMUP and CMX muons. Lower plots are muon hadron calorimeter energy deposition distributions for CMUP and CMX muons. 

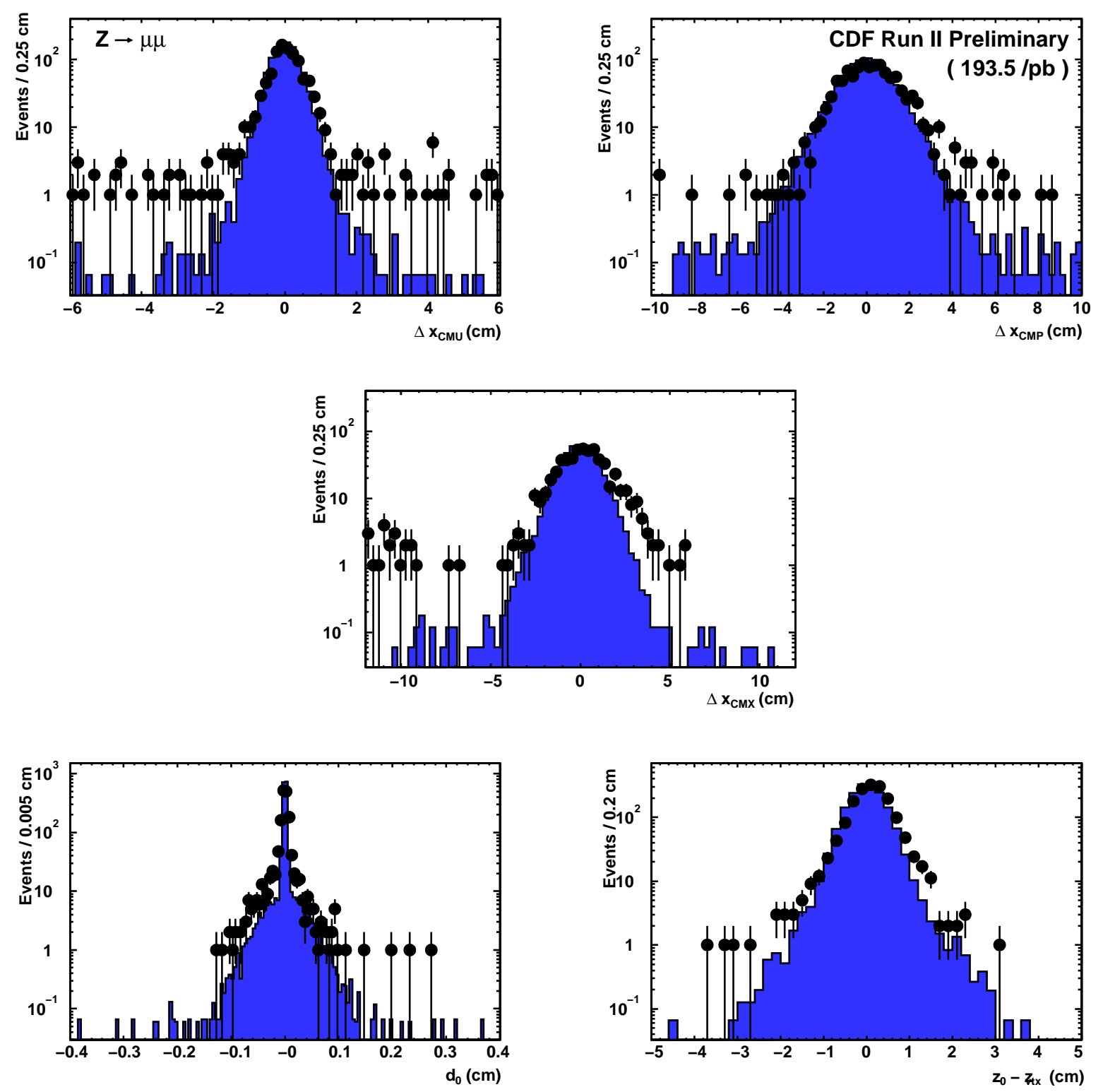

Figure 3.14: The muon variables used for the selection of the events. The tight muon in the $Z \rightarrow \mu^{+} \mu^{-}$candidates events (dots) and in the Monte Carlo (solid histogram) are used. For each variable all the selection criteria, but the one including the variable itself, are applied. The number of Monte Carlo events is normalized to the $193.5 \mathrm{fb}^{-1}$ data. Upper and middle plots are track-stub matching $\Delta x=r \times \Delta \phi$ for CMUP and CMX muons. Lower plots are impact parameter $d_{0}$ and $z_{0}-z_{v t x}$ distributions. 


\subsection{Triggers and Datasets}

We expect a high- $p_{T}$ lepton in the final state because the like-sign signature requires the on-shell $W$ produced in association with a Higgs boson to decay semileptonically. The inclusive high- $p_{T}$ lepton triggers are thus a natural choice for the analysis. They are

- ELECTRON_CENTRAL_18 [53],

1. Level 1 trigger : L1_CEM8_PT8, CEM tower with $E_{\mathrm{T}}>8.0 \mathrm{GeV}$ and XFT track matched tower with $P_{\mathrm{T}}>8.0 \mathrm{GeV}$,

2. Level 2 trigger : L2_CEM16_PT8, CEM cluster with $E_{\mathrm{T}}>16.0 \mathrm{GeV}$, XFT track matched cluster with $8.0 \mathrm{GeV}$ and $E_{\text {had }} / E_{\text {em }}<0.125$,

3. Level 3 trigger : L3_ELECTRON_CENTRAL_18, L3 calorimeter cluster with $E_{\mathrm{T}}>18.0 \mathrm{GeV}$ and COT track matching the cluster with $P_{\mathrm{T}}>9.0 \mathrm{GeV}$,

- MUON_CMUP18 [64],

1. Level 1 trigger : L1_CMUP6_PT4, CMU stub with $P_{\mathrm{T}}>6.0 \mathrm{GeV}, \mathrm{XFT}$ track with $P_{\mathrm{T}}>4.0 \mathrm{GeV}$ and matched CMP muon,

2. Level 2 trigger : L2_AUTO_L1_CMUP6_PT4 or L2_TRK8_L1_CMUP6_PT4, L1 auto accept or XFT track with $P_{\mathrm{T}}>4.0 \mathrm{GeV}$,

3. Level 3 trigger : L3_MUON_CMUP18, L3 CMP muon with $\left|d_{\mathrm{x}}\right|<20 \mathrm{~cm}$, L3 CMU muon with $\left|d_{\mathrm{x}}\right|<10 \mathrm{~cm}$, and L3 track with $P_{\mathrm{T}}>18.0 \mathrm{GeV}$.

- MUON_CMX18 [64].

1. Level 1 trigger : L1_CMX6_PT8_PS1, L1_CMX6_PT8_CSX_PS1 or L1_CMX6_PT8_CSX, CMX stub with $P_{\mathrm{T}}>6.0 \mathrm{GeV}$, XFT track with $P_{\mathrm{T}}>8.0 \mathrm{GeV}$ and matched CSX muon,

2. Level 2 trigger : L2_AUTO_L1_CMX6_PT4 or L2_AUTO_L1_CMX6_PT8_CSX, L1 auto accept,

3. Level 3 trigger : L3_MUON_CMX18 .

L3 CMX muon with $\left|d_{\mathrm{x}}\right|<10 \mathrm{~cm}$, and L3 track with $P_{\mathrm{T}}>18.0 \mathrm{GeV}$. 
Our data samples originate from the Stream-B inclusive high- $p_{T}$ electron and muon datasets. The datasets were reduced with loose lepton-identification cuts, and further processed by the CDF Top group to make corrections on tracking and calorimeter information ("remake dataset") [65]. These remake-datasets are our initial data samples. The trigger efficiencies are described in section 6.7 .

\subsubsection{Good Runs and Integrated Luminosity}

Good-run status and luminosity information for each run are provided in the GOOD RUN LIST web page [67]. The good runs with "no silicon" in the range 141544-168889 (from Mar-02 to Sep-03) are selected. The integrated luminosity is $(193.5 \pm 11.4) \mathrm{pb}^{-1}$ for the ELECTRON_CENTRAL_18 and MUON_CMUP18 triggers, while it is $(175.3 \pm 10.3) \mathrm{pb}^{-1}$ for the MUON_CMX18 trigger.

\subsubsection{Inclusive High $p_{T}$ Lepton samples}

Inclusive high $p_{T}$ leptons are produced in hadron collisions in decays of the electroweak bosons such as $W \rightarrow \ell \nu$ and $Z \rightarrow \ell \ell$ and also dilepton decay from onium resonances around $10<M_{\text {reso }}<12 \mathrm{GeV} / c^{2}$ by the above triggers. High $p_{T}$ electron clusters or muon are also produced in QCD processes, where the electron or muon is embedded in a high $p_{T}$ jet of hadrons. The processes in which hadronic jets can produce an electron cluster are:

- electrons which come in $e^{+} e^{-}$pairs, either from photon conversions or Dalitz decays [66],

- semileptonic decays of heavy quarks,

- fake electron clusters which are really hadron showers that pass our electron identification cuts: e.g. overlaps of $\pi^{ \pm}$and $\pi^{0}$ showers.

For muon:

- Punch through hadrons, which pass through the electromagnetic and hadron calorimeter and make hits to the muon detectors,

- Decay-in-flight muon in the reaction: $\pi^{ \pm}, K^{ \pm} \rightarrow \mu \nu$,

Among the about $200 \mathrm{pb}^{-1}$ of data, additional selection criteria are applied to obtain a sample of events with leptons which is referred to as a "tight" central lepton to select events satisfying the trigger. Requiring tight cuts on a central electron and muon serves three purposes. First, we must select at least one lepton object satisfying the trigger described before. Second, this well-understood central region has added 
information from the tracking and the strip chambers that can be used to suppress background from other physics processes. Third, the tight cuts on the central electron allow loose, highly efficient cuts to be placed on the second lepton. The criteria used in this analysis are listed in Table 3.2. And leptons (electron and muon) produced by QCD processes are not expected to have low isolation e.g. from QCD jets faking an electron and semileptonic decays of heavy quarks. The isolation in lepton selection is one of the powerful cuts to suppress the fake objects. The $E_{T}$ and $p_{T}$ spectra of the electrons in the inclusive, tight and isolated samples are shown in Figure 3.15. A peak from the Jacobian of the $W$ and $Z$ is already apparent.

\begin{tabular}{|c|c|}
\hline \multicolumn{2}{|c|}{$\frac{\text { Event vertex cut }}{\left|z_{\text {vtx }}\right|<60 \mathrm{~cm}}$} \\
\hline Electron selection & Muon selection \\
\hline \multicolumn{2}{|c|}{ Geometrical and kinematical cuts } \\
\hline \multirow{3}{*}{$\begin{array}{l}\text { CEM } \\
\text { Fiducial } \\
E_{T}^{\ell_{1}}>20 \mathrm{GeV}\left(p_{T}>10 \mathrm{GeV} / c\right)\end{array}$} & CMUP or CMX \\
\hline & Fiducial (CMUP), $\rho_{\mathrm{COT}}>140 \mathrm{~cm}(\mathrm{CMX})$ \\
\hline & $p_{T}^{\ell_{1}}>20 \mathrm{GeV} / c$ \\
\hline \\
\hline \multicolumn{2}{|c|}{$I S O_{0.4}^{\mathrm{cal}}<2 \mathrm{GeV}$} \\
\hline \multicolumn{2}{|c|}{ Identification cuts } \\
\hline $\begin{array}{l}\mathrm{HAD} / \mathrm{EM}<0.055+0.00045 \times E \\
L_{\mathrm{shr}}<0.2\left(E_{T}<70 \mathrm{GeV}\right) \\
E / p<2\left(E_{T}<50 \mathrm{GeV}\right) \\
\chi_{\text {strip }}^{2}<10 \\
\left|\Delta z_{\mathrm{CES}}\right|<3 \mathrm{~cm} \\
-3.0<Q \times \Delta x_{\mathrm{CES}}<1.5 \mathrm{~cm}\end{array}$ & $\begin{array}{l}\mathrm{EM}<\max (2,2+0.0115 \times(p-100)) \mathrm{GeV} \\
\mathrm{HAD}<\max (6,6+0.0280 \times(p-100)) \mathrm{GeV} \\
|r \times \Delta \phi|<3,5,6 \mathrm{~cm}(\mathrm{CMU}, \mathrm{P}, \mathrm{X})\end{array}$ \\
\hline \multicolumn{2}{|c|}{$\begin{array}{l}\text { Track quality: } \text { stereo } \geq 3 \text { and axial } \geq 3, \geq 7 \text { hits } \\
\qquad\left|z_{0}-z_{\mathrm{vtx}}\right|<2 \mathrm{~cm} \\
\left.\left|d_{0}\right|<0.2 \mathrm{~cm} \text { (silicon hits }<3\right), 0.02 \mathrm{~cm}(\geq 3)\end{array}$} \\
\hline Conversion removal & \\
\hline
\end{tabular}

Table 3.2: Criteria for central tight electron and muon candidates 

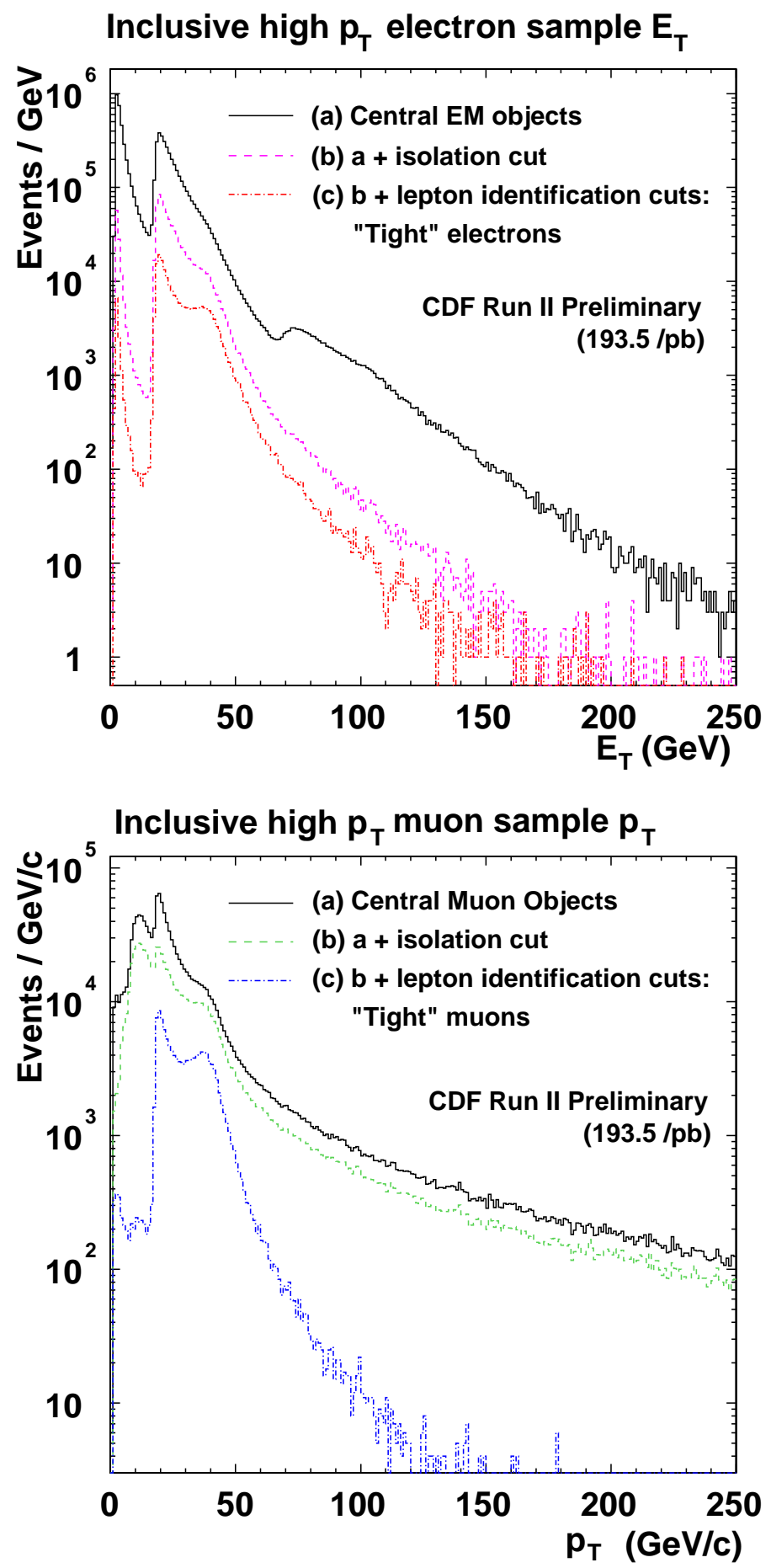

Figure 3.15: Upper distributions are transverse energy for all electrons in the inclusive high $p_{T}$ electron sample. Lower distributions are transverse momentum for all muons in the inclusive high $p_{T}$ muon sample. 


\subsection{Selection Cuts}

Our idea is to select high- $p_{T}$ isolated like-sign events with simple requirements but with enough cleanliness, and understand them in terms of backgrounds rather than jumping directly to background estimation in the final sample. The event selection therefore consists mostly of the "standard", maybe referred to as "tight" also, criteria and even tries to allow more events to remain in the sample by keeping kinematical cuts on the 2nd leading lepton-leg at minimal.

Table 3.3 lists our primary-vertex and lepton selection cuts. The vertex with the highest $p_{T}$ sum of associated tracks is chosen and required to be within the region to ensure well-defined measurements of collisions by the detector. The leptons we identity are checked if they are attached to this primary-vertex. The CEM, CMUP, and CMX are currently allowed for both lepton legs. We require at least one electron (muon) with $E_{T}\left(p_{T}\right)>20 \mathrm{GeV}(\mathrm{GeV} / c)$ which is considered to be responsible for firing the trigger(s) we have chosen, and at least one other electron (muon) with $E_{T}\left(p_{T}\right)>6 \mathrm{GeV}$ $(\mathrm{GeV} / c)$. The particular choice of $6 \mathrm{GeV}(/ c)$ on the 2nd lepton is rather arbitrary: we just pick up the value used in a Run-I like-sign analysis [68]. The EM energy correction is applied to electron $E_{T}$ 's, and lepton momenta are derived with the beam-constraint fit. The leptons are required to be isolated in terms of the traditional calorimeter cone-isolation with a cone size of $R=0.4\left(I S O_{0.4}^{\text {cal }}\right)$. To clarify, we comment that the $E / p$ in the electron selection is not $E_{T} / p_{T}$. The impact parameter $\left(d_{0}\right)$ is recalculated with respect to the primary vertex, and the track $z_{0}$-parameter is also recalculated to take the $z$ coordinate of the closest-approach point with respect to run-averaged beam line, although its effect is small in the latter. The impact parameter cut for electrons might probably be unusual. We make it in the electron selection because we estimate fake-lepton backgrounds using per-track fake rates in the main analysis, and keeping the same track-selection between electrons and muons simplifies the study. We reject electrons as conversion candidates if an oppositely-charged track satisfying

$$
\left|\delta_{x y}\right|<0.2 \mathrm{~cm} \text { and }|\Delta(\cot \theta)|<0.04
$$

is found in the track-object list. The details of each lepton selection cut value were described in section 3.1.3 and 3.1.4.

For the events with $\geq 2$ leptons passing the above selection, we apply the cuts listed in Table 3.4 to clean up the sample. We note that the like-sign requirement comes last, so the other cuts are commonly applied to both charge-combinations for simplicity. We use the standard cosmic-tagger module [69] for vetoing cosmic rays. The leptons must be consistent with coming from the same vertex, which is an important requirement for multi-lepton signatures. We also require a dilepton mass cut to avoid seeing oniums produced with the rates that we do not understand well. An algorithm of removing $Z$ is implemented aiming mainly to reduce $W Z$ backgrounds which are irreducible in our channel. Rejection requires efforts to widen the acceptance of $Z$ legs, including 
plug electrons for example, since events would seemingly represent like-sign signatures otherwise. For each lepton identified with our selection, the algorithm looks for a high$p_{T}$ isolated track with oppositely charged or a loosely-identified lepton of the same type but without asking charge combination, then discards the event if the invariant mass falls in a $Z$ mass window between $81 \mathrm{GeV} / c^{2}$ and $101 \mathrm{GeV} / c^{2}$. The criteria for identifying these 2 nd objects are listed in Table 3.5, which basically follows the one used in the Top group [70]. The application of $Z$ removal to like-sign lepton-pairs, dielectrons particularly, also rejects contributions originally from opposite-sign pairs with either incorrect charge measurements or hard photon-conversions. We finally require at least one like-sign pair in the event and let the events with $\geq 3$ leptons remain in our sample. The detail of each lepton selection cut value are described in section 6.5. The background estimation are described in chapter 4 .

\subsection{Survived Events}

Since there is a possibility for us to find same $e \mu$ events from the electron-trigger path and the muon-trigger path, we explicitly check this point to make sure no doublecount. In Table 3.6, we show event reduction by the cuts on lepton pairs, where the $e \mu$ $(\mu e)$ in the table is meant to indicate that the leading lepton is the electron (muon).

Distributions of $p_{T}, \eta$, and $I S O_{0.4}^{\text {cal }}$ for the 2nd lepton-leg are shown in Figure 3.16. Figure 3.17 shows distributions of $\mathscr{E}_{T}, \cos \phi_{12}$, and $p_{T 12}$, where $\phi_{12}$ is the azimuthal

opening-angle between two like-sign leptons and $p_{T_{12}}$ is the $p_{T}$ of the vector sum of two leptons' momenta.

\subsection{Summary of Event Selection}

We summarized the like-sign dilepton selection from high- $p_{T}$ inclusive lepton datasets which we analyze for our neutral Higgs boson search. The selection identifies high- $p_{T}$, isolated leptons with almost "standard" identification criteria. We observed 45 like-sign dilepton events in about $200 \mathrm{pb}^{-1}$ data. 

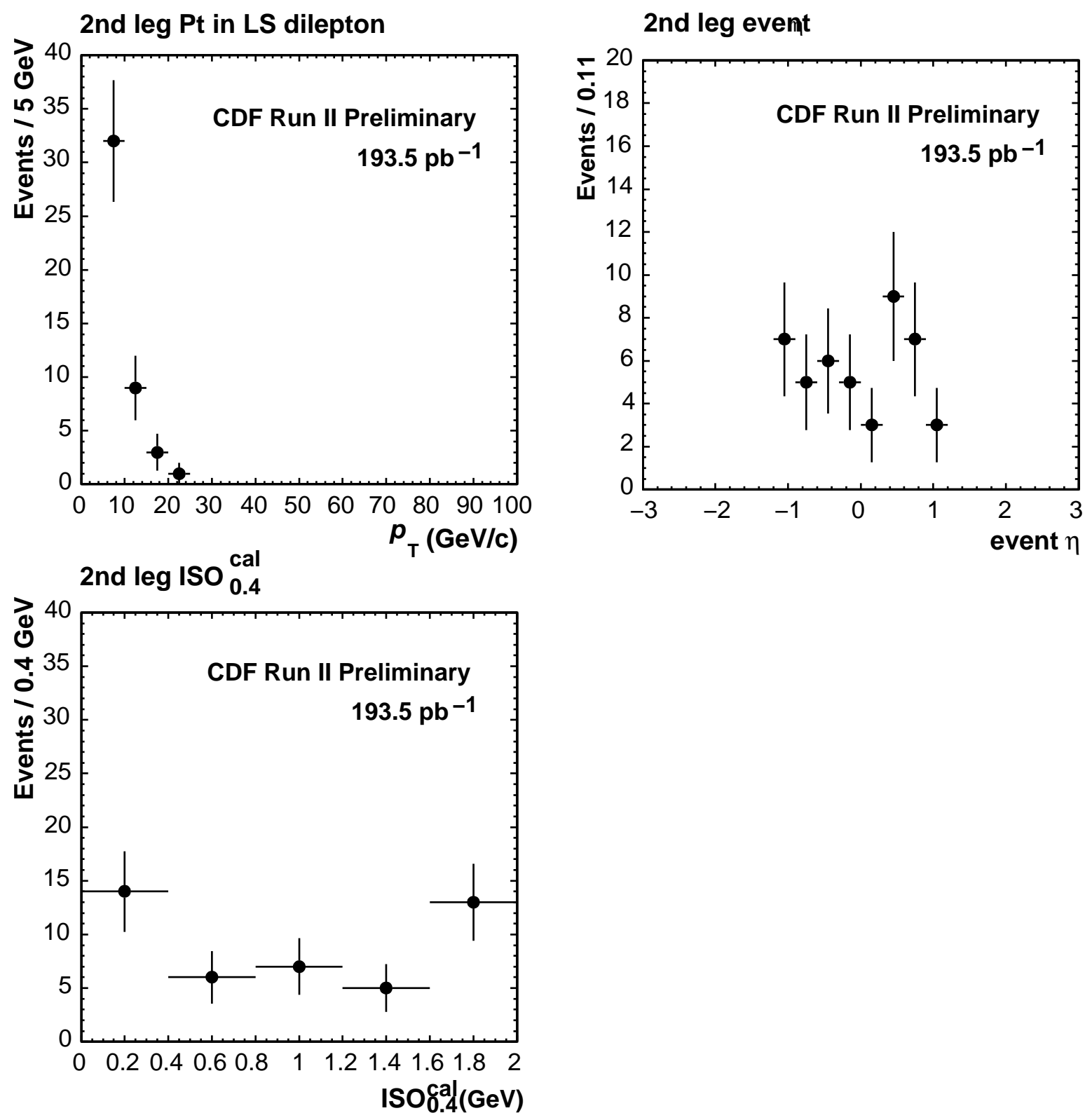

Figure 3.16: Distributions of $p_{T}$, event $\eta$, and $I S O_{0.4}^{\text {cal }}$ for the 2 nd lepton leg in the like-sign dilepton events selected from inclusive high- $p_{T}$ lepton data. 

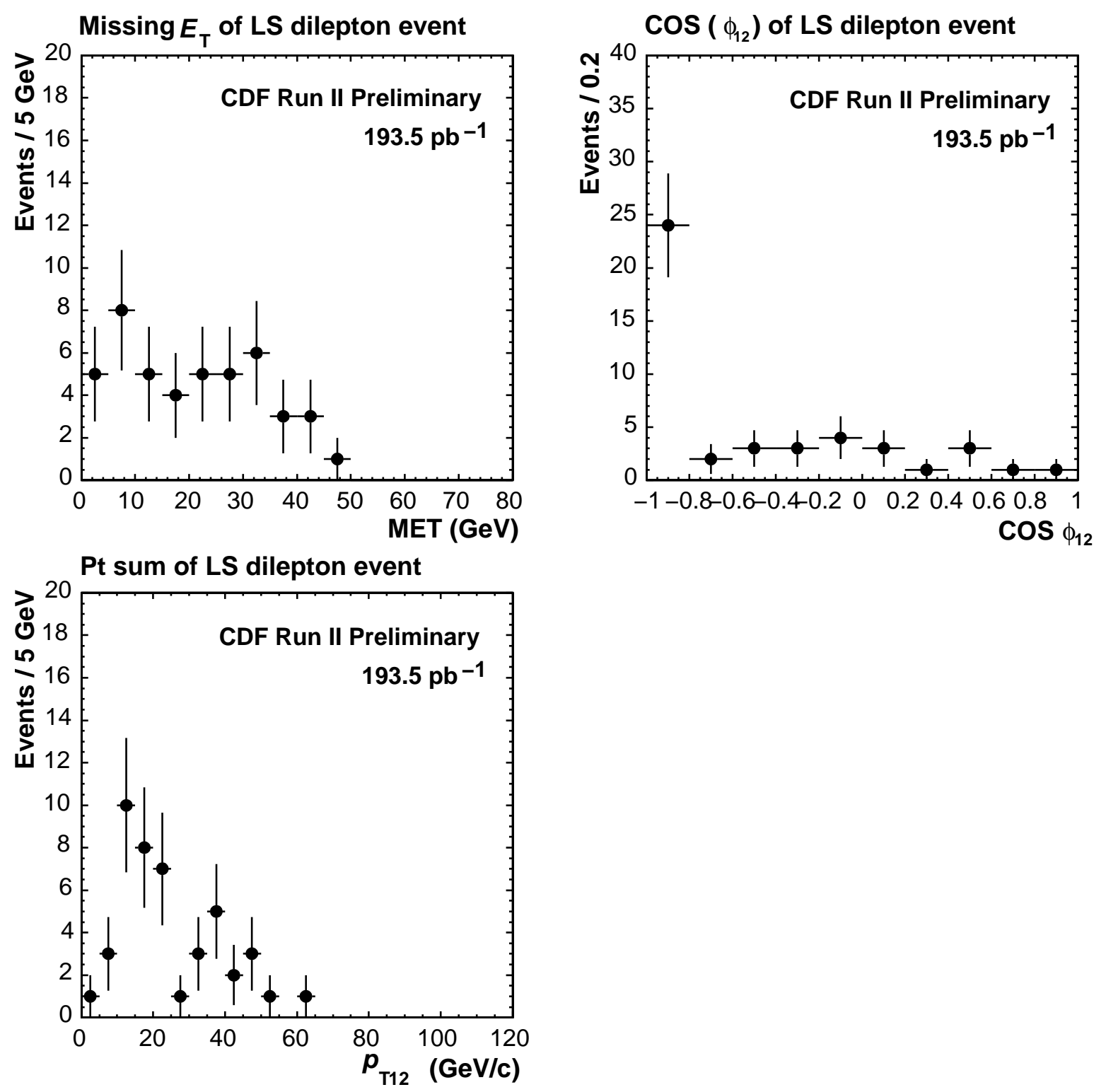

Figure 3.17: Distributions of $\mathbb{E}_{T}, \cos \phi_{12}$, and $p_{T 12}$ for the like-sign dilepton events selected from inclusive high- $p_{T}$ lepton data. 


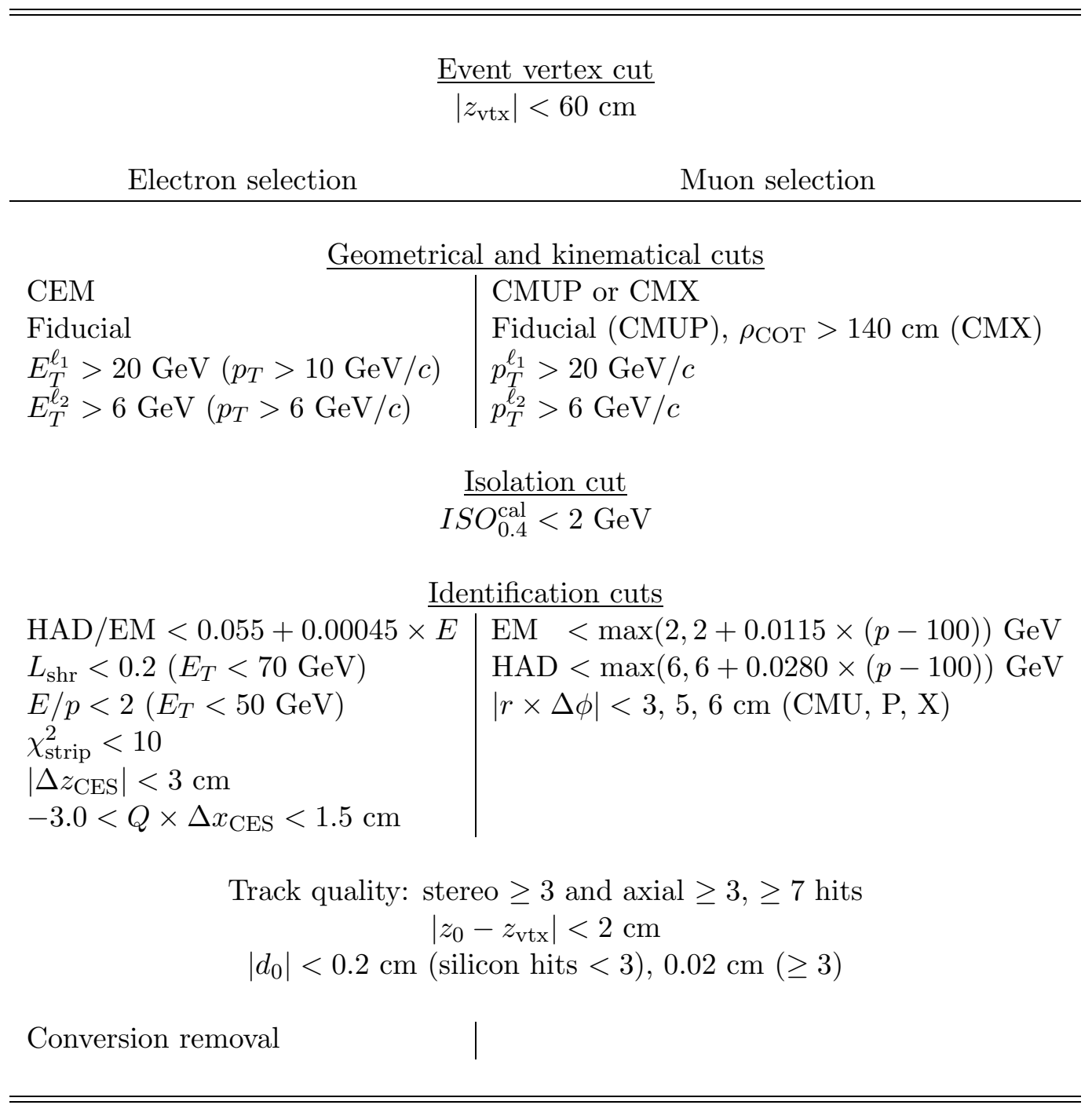

Table 3.3: Primary vertex and lepton selection cuts.

\begin{tabular}{l}
\hline No cosmic-flag \\
$\left|z_{0}^{\ell_{1}}-z_{0}^{\ell_{2}}\right|<2 \mathrm{~cm}$ \\
Dilepton mass $>12 \mathrm{GeV}$ \\
$Z$ removal \\
At least one like-sign pair \\
\hline \hline
\end{tabular}

Table 3.4: Dilepton selection cuts. 


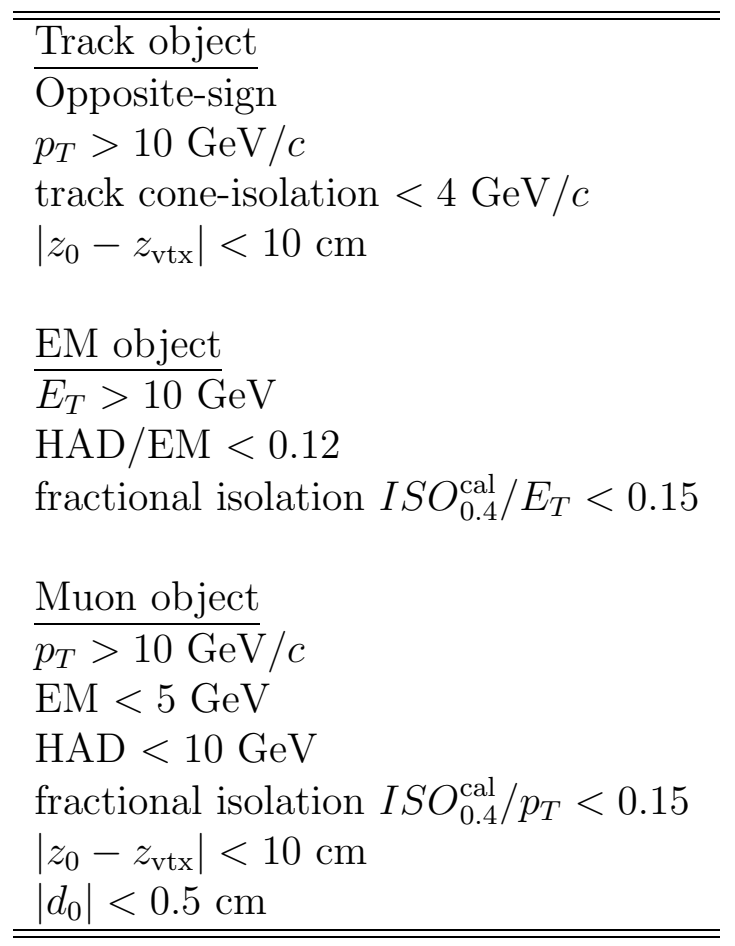

Table 3.5: Physics objects used to identify and remove $Z$ bosons.

\begin{tabular}{l|rrrr|r} 
& \multicolumn{5}{c}{ CDF Run II Preliminary $\left(193.5 \mathrm{pb}^{-1}\right)$} \\
\hline \hline & $e e$ & $e \mu$ & $\mu e$ & $\mu \mu$ & Total \\
\hline Dilepton candidates & 2914 & 56 & 64 & 2394 & 5428 \\
No cosmic-flag & 2914 & 55 & 64 & 2260 & 5293 \\
$\left|z_{0}^{\ell_{1}}-z_{0}^{\ell_{2}}\right|<2 \mathrm{~cm}$ & 2895 & 55 & 63 & 2246 & 5259 \\
Dilepton mass $>12 \mathrm{GeV}$ & 2839 & 55 & 63 & 2099 & 5056 \\
$Z$ removal & 637 & 54 & 62 & 808 & 1561 \\
Like-sign & 16 & 11 & 13 & 5 & 45 \\
\hline \hline
\end{tabular}

Table 3.6: Number of events passing like-sign dilepton selection. 


\section{Chapter 4}

\section{Background Estimation for High- $p_{T}$ Like-Sign Dilepton}

Although the like-sign requirement is quite effective to suppress QCD and known electroweak processes, we expect that the fake lepton background still remains at the considerable level in the events of our signature. We measure the fake rate per isolated-track using the JET samples in Section 4.2 in order to estimate the fake lepton background. We also note that the residual photon conversions, which are defined as electrons from photon conversions with unobserved partner tracks, are not negligible. We estimate the tagging efficiency of the photon conversion events to obtain the contribution of the residual photon conversions in Section 4.3. We, then, separate its contribution from the fake leptons in Section 4.4. Finally we compare the number of expected events with that of observed events for the lepton samples collected with three different triggers in Section 4.5. In this chapter we use the selection cuts of the high- $p_{T}$ isolated like-sign dilepton which are described in the previous chapter.

\subsection{Monte Carlo Background Samples}

We use Monte Carlo samples to estimate backgrounds except for the fake leptons and the residual photon conversions. These samples were created by the Top and Electroweak groups. The backgrounds are classified into irreducible and reducible backgrounds:

- Irreducible backgrounds:

1. $W Z \rightarrow \ell^{ \pm} \nu \ell^{-} \ell^{+}$,

2. $Z Z \rightarrow \ell^{-} \ell^{+} \ell^{-} \ell^{+}$.

- Reducible backgrounds: 


\begin{tabular}{c|ccc}
\hline \hline Process & $N_{\text {gen }}$ & $\sigma \cdot B r(\mathrm{pb})$ & Comment \\
\hline$W^{ \pm} Z, Z \rightarrow \ell \ell$ & $990 \mathrm{~K}$ & 0.244 & {$[73]$} \\
$Z Z$ & $240 \mathrm{~K}$ & $0.994^{*}$ & {$[73]$} \\
$t t \rightarrow$ dilepton & $100 \mathrm{~K}$ & 1.27 & $p_{T}^{\text {gen }}>8 \mathrm{GeV} / c$ lepton pair \\
$W b b \rightarrow \ell \nu b b$ & $230 \mathrm{~K}(W \rightarrow e \nu)$ & 2.91 & {$[73]$} \\
& $220 \mathrm{~K}(W \rightarrow \mu \nu)$ & 2.91 & {$[73]$} \\
$W c c \rightarrow \ell \nu c c$ & $250 \mathrm{~K}(W \rightarrow \tau \nu)$ & 2.91 & {$[73]$} \\
& $270 \mathrm{~K}(W \rightarrow e \nu)$ & 4.75 & {$[73]$} \\
& $300 \mathrm{~K}(W \rightarrow \mu \nu)$ & 4.75 & {$[73]$} \\
$\gamma^{*} / Z \rightarrow \ell^{-} \ell^{+}$ & $300 \mathrm{~K}(W \rightarrow \tau \nu)$ & 4.75 & {$[73]$} \\
& $3 \mathrm{M}(e e)$ & 269.3 & $M_{\ell \ell}>30 \mathrm{GeV} / c^{2},[74]$ \\
$W W \rightarrow \ell \nu \mathrm{xx}$ & $3 \mathrm{M}(\mu \mu)$ & 249.9 & $M_{\ell \ell}>30 \mathrm{GeV} / c^{2},[75]$ \\
& $2 \mathrm{M}(\tau \tau)$ & 269.3 & $M_{\ell \ell}>30 \mathrm{GeV} / c^{2},[74]$ \\
\hline \hline
\end{tabular}

* production cross section only.

Table 4.1: Summary of the Monte Carlo samples; number of generated events, accepted cross section $\sigma \cdot B r(\mathrm{pb})$, and comments.

1. $t \bar{t} \rightarrow W^{+} b W^{-} \bar{b} \rightarrow$ Like-sign dilepton

(One lepton comes from $W$ and other comes from semileptonic decay of heavy flavor quarks),

2. $W b \bar{b}, W c \bar{c} \rightarrow$ Like-sign dilepton

(The same combination of $t \bar{t}$ decay),

3. $\gamma^{*} / Z \rightarrow \ell^{-} \ell^{+}$,

4. $W W \rightarrow \ell^{-} \nu \ell^{+} \bar{\nu}$.

Table 4.1 shows the summary of the Monte Carlo samples for the above backgrounds. The accepted cross section of the irreducible diboson backgrounds, $W Z$ and $Z Z$, are small (about $10^{-1} \mathrm{pb}$ ) due to the small cross section of the diboson production and also the small branching ratio of the leptonic decay of vector bosons. The reducible QCD and electroweak processes are strongly suppressed by the isolation cut and the like-sign requirement.

\subsection{Fake Lepton Background}

The lepton plus fake lepton background arises from a single lepton event such as $W \rightarrow l \nu$. This type of background consists of one trigger lepton and one fake lepton. The physics process components of the fake lepton are listed below : 
- Fake leptons :

1. Interactive $\pi^{ \pm} \rightarrow$ fake electron,

2. Overlap $\left(\pi^{0}+\right.$ track $) \rightarrow$ fake electron,

3. Punch through hadron $\rightarrow$ fake muon.

- Non-prompt leptons :

1. Residual photon conversion $\rightarrow$ electron,

2. Decay-in-fight muon $\left(\pi^{ \pm}, K^{ \pm} \rightarrow \mu^{ \pm} \nu\right) \rightarrow$ muon,

3. Heavy flavor jet $\rightarrow$ bad-isolation lepton.

The amount of the above background is estimated from the fake rate. We define the fake rate $R_{\text {fake }}$ as a rate for the isolated tracks to pass the lepton identification cuts:

$$
R_{\text {fake }}=\frac{\text { Number of isolated tracks which pass the lepton identification cuts }}{\text { Number of isolated tracks }} .
$$

It is noted that we define the fake rate here per isolated track, not per jet. We evaluate $R_{\text {fake }}$ using the JET samples by assuming that the components of the fake lepton are the same between the JET samples and the like-sign dilepton events in the inclusive lepton sample. We, then, select events which contain the lepton plus isolated tracks, and the events are scaled by the fake rate to give the number of expected fake lepton events. We note that the residual photon conversion component will be separated from the fake lepton background in Section 4.3 .

\subsubsection{Lepton Fake-Rate Measurement}

An isolated track is defined by the following criteria [71]:

- $p_{T}>6.0 \mathrm{GeV} / c$ and $|\eta|<1.1$,

- $I S O_{0.4}^{c a l}<2.0 \mathrm{GeV}$,

- Track quality cut (stereo $\geq 3$ and axial $\geq 3$ with $\geq 7$ hits),

- $\left|z_{0}-z_{\mathrm{vtx}}\right|<2.0 \mathrm{~cm}$,

- $\left|d_{0}\right|<0.2 \mathrm{~cm}$ (SI hit $<3$ ), $0.02 \mathrm{~cm}$ (SI hit $\geq 3$ ).

We also require a separation between the trigger jet and the isolated track in $\eta$ - $\phi$ space to be $\Delta R>1.0$ to remove the trigger bias, as well as a series of cuts for high- $p_{T}$ real lepton removal such as $W$ veto $\left(\mathbb{E}_{T}<30 \mathrm{GeV}\right.$ and transverse mass $\left.<40 \mathrm{GeV} / c^{2}\right), Z$ veto, and cosmic veto. We use the JET20 and JET50 samples to measure the fake rate. Figures 4.1 and 4.2 show the $p_{T}$ and $I S O_{0.4}^{\text {cal }}$ distributions of isolated tracks, electrons, 
and muons. Here an "electron" and a "muon" mean the isolated tracks passing the identification cuts for each lepton. We calculate the fake rate in accordance with the definition in Equation 4.1, and the obtained results are shown in Figures 4.3 and 4.4 in terms of the $p_{T}$ and $I S O_{0.4}^{c a l}$ dependences.

We consider think over two different sources of the fake rate systematic error. The first systematic error is sample dependence of two jet samples. We take the half of difference between two jet samples as one of the systematic error. The second one is systematic of a bias from trigger jet. We require the separation cut between trigger jet and isolated track to select isolated track. Since in jet samples there are many two-jet events which are balanced, the isolated tracks which are about $\pi$ away from trigger jet have a potential of the bias due to the the trigger jet's $E_{T}$ dependence. In Figures 4.5, 4.6, and 4.7 we show the $\cos \phi_{12}$ distribution and $\cos \phi_{12}$ dependence of the fake rate, where $\phi_{12}$ is $\phi$ separation between isolated track and trigger jet. Although we don't see the obvious trigger bias in the low $\cos \phi_{12}$ bins, we consider the half of difference of fake rate between $\cos \phi_{12} \geq-0.8$ region and $\cos \phi_{12}<-0.8$ region as a systematic error of trigger bias.

\subsubsection{Validation of the Lepton Fake Rate}

We look at the JET70, JET100, and inclusive high- $E_{T}$ photon samples for validation of the lepton fake rate. We compare the leptons expected from the fake rate with the observed leptons for the above three samples. The expected and observed lepton $p_{T}$

and $I S O_{0.4}^{c a l}$ distributions are shown in Figure 4.8, 4.9, and 4.10. We see reasonable agreements for each sample within the estimated error. 

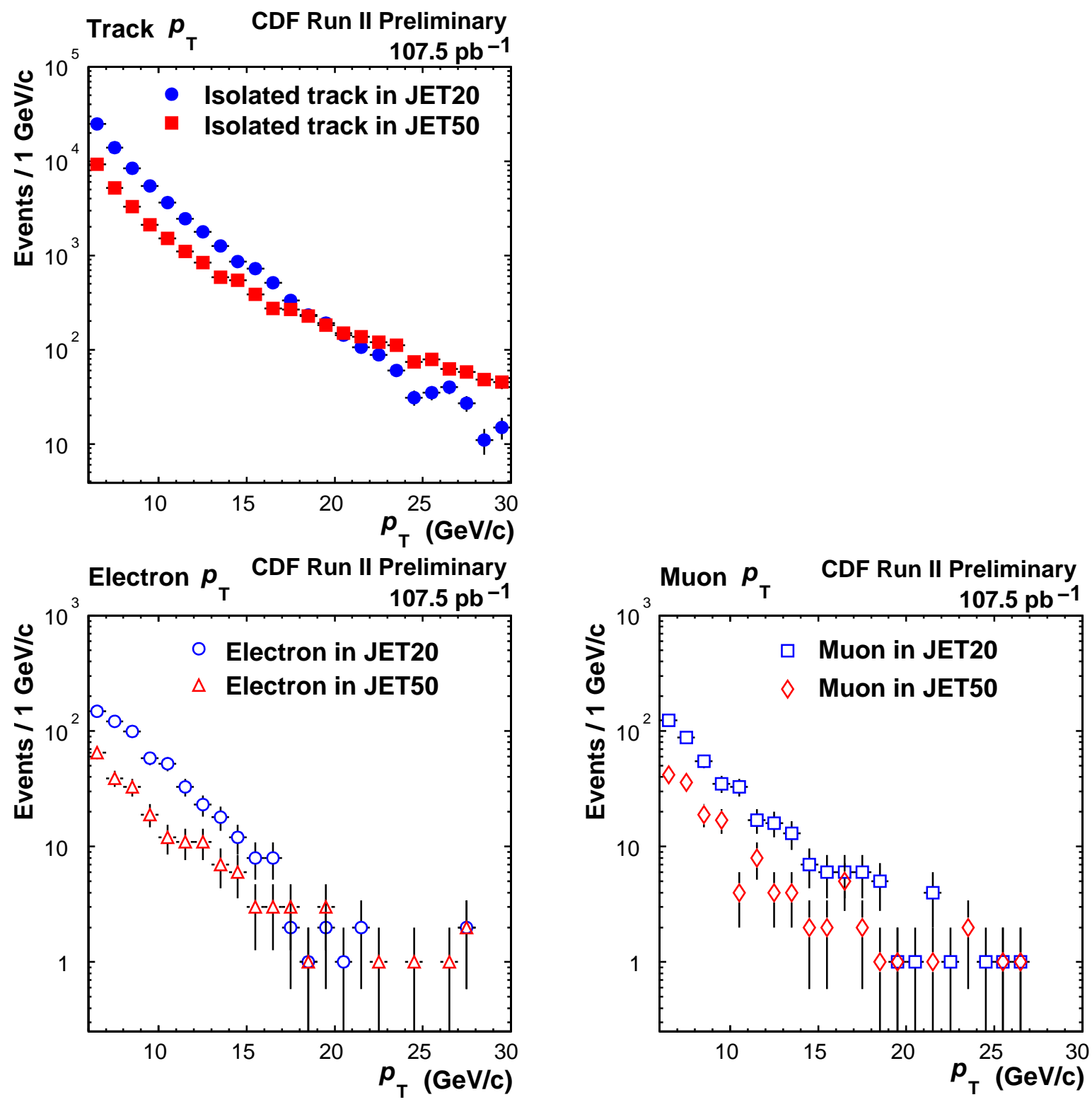

Figure 4.1: Track $p_{T}$ distributions of isolated tracks (upper left), electrons (lower left), and muons (lower right) in the JET samples. Points correspond to JET20 and JET50 samples. 

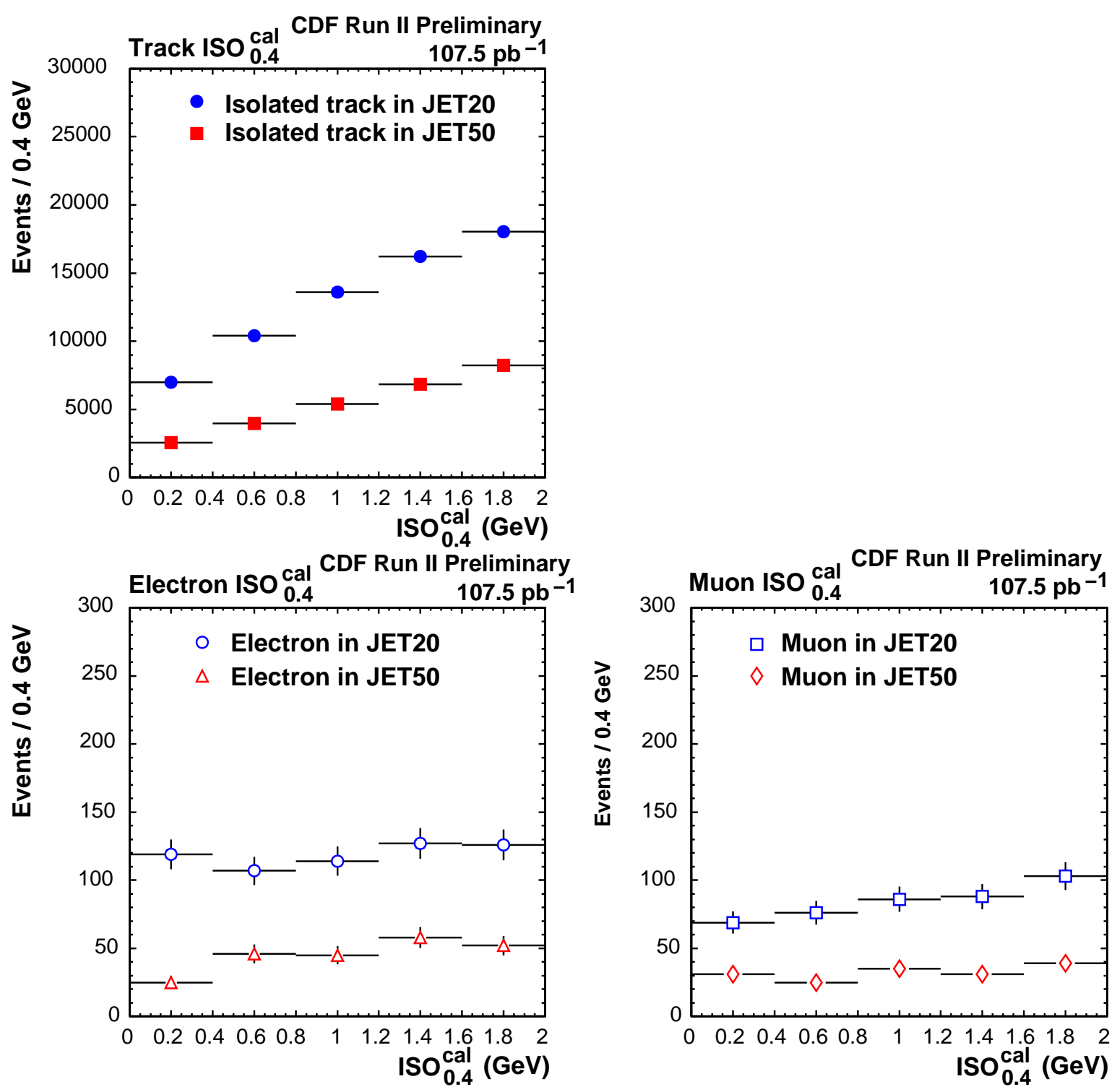

Figure 4.2: Cone 0.4 calorimeter isolation $I S O_{0.4}^{c a l}$ distributions of isolated tracks (upper left), electrons (lower left), and muons (lower right) in the JET samples. Points correspond to JET20 and JET50 samples. 

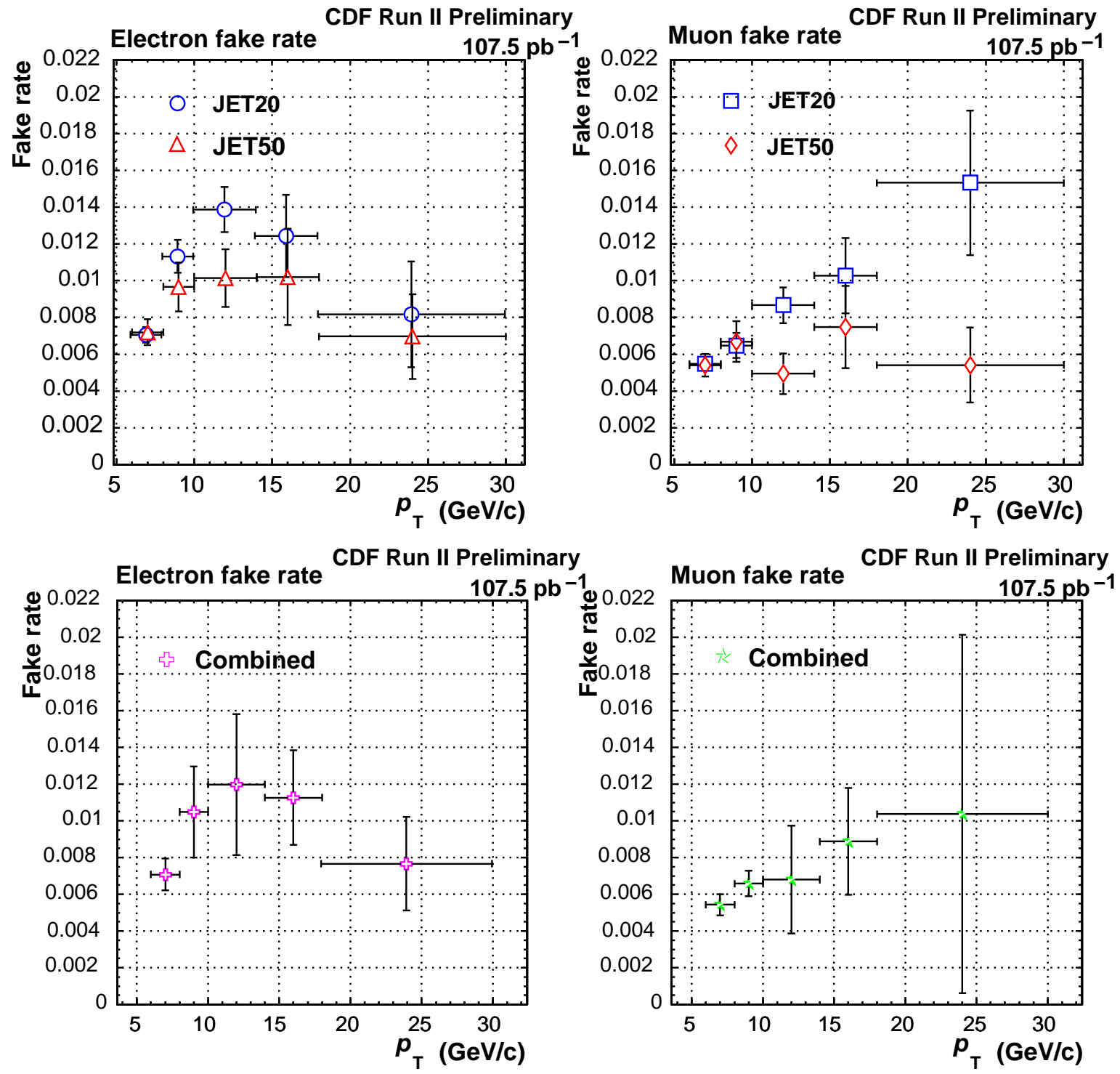

Figure 4.3: Track $p_{T}$ dependence of the lepton fake rate. The upper left (right) plot shows the electron (muon) fake rate for the JET20 and JET50 samples. The lower left (right) plot shows the combined electron (muon) fake rate. 

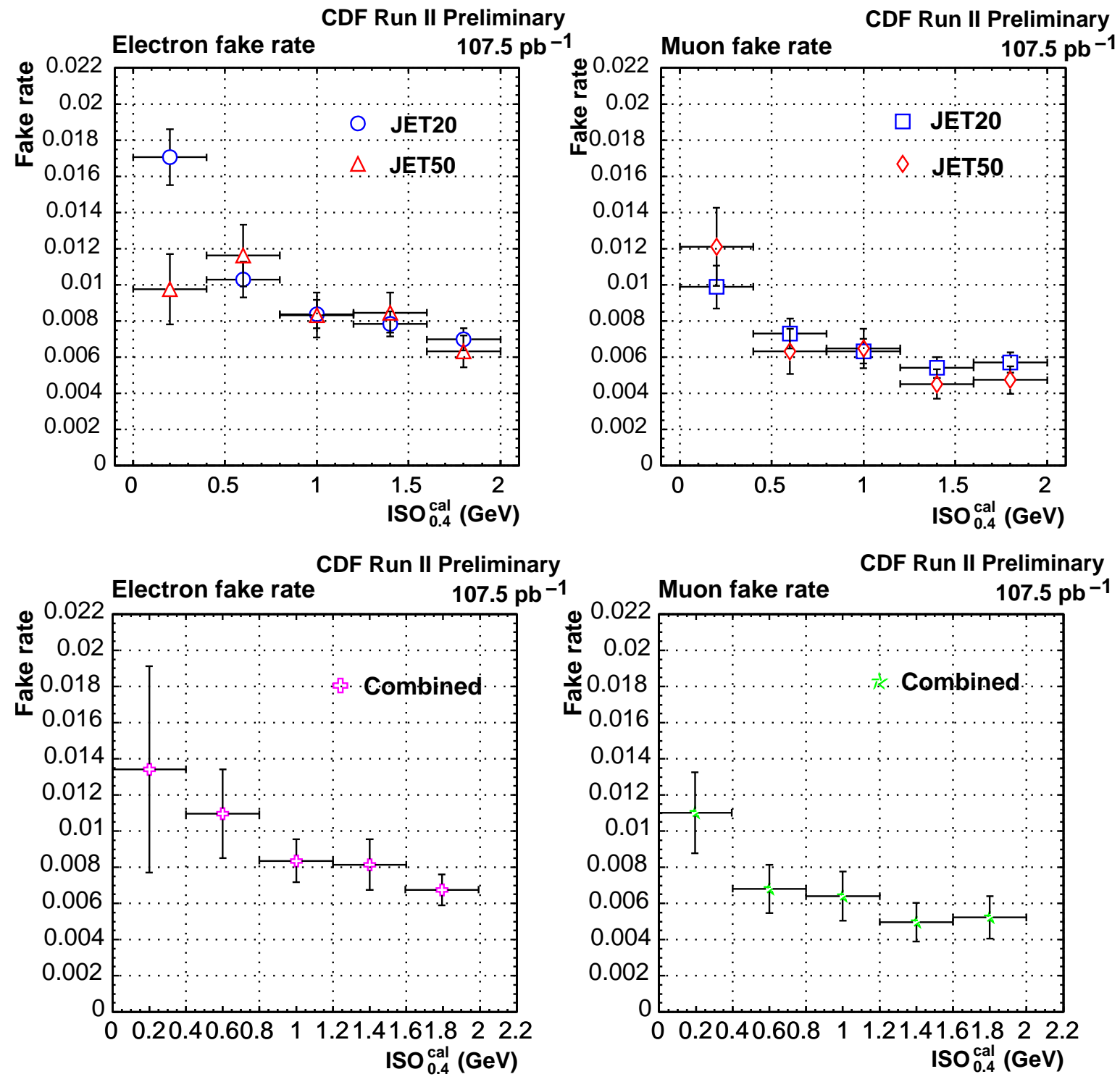

Figure 4.4: Cone 0.4 calorimeter isolation $I S O_{0.4}^{c a l}$ dependence of the lepton fake rate. The upper left (right) plot shows the electron (muon) fake rate for the JET20 and JET50 samples. The lower left (right) plot shows the combined electron (muon) fake rate. 

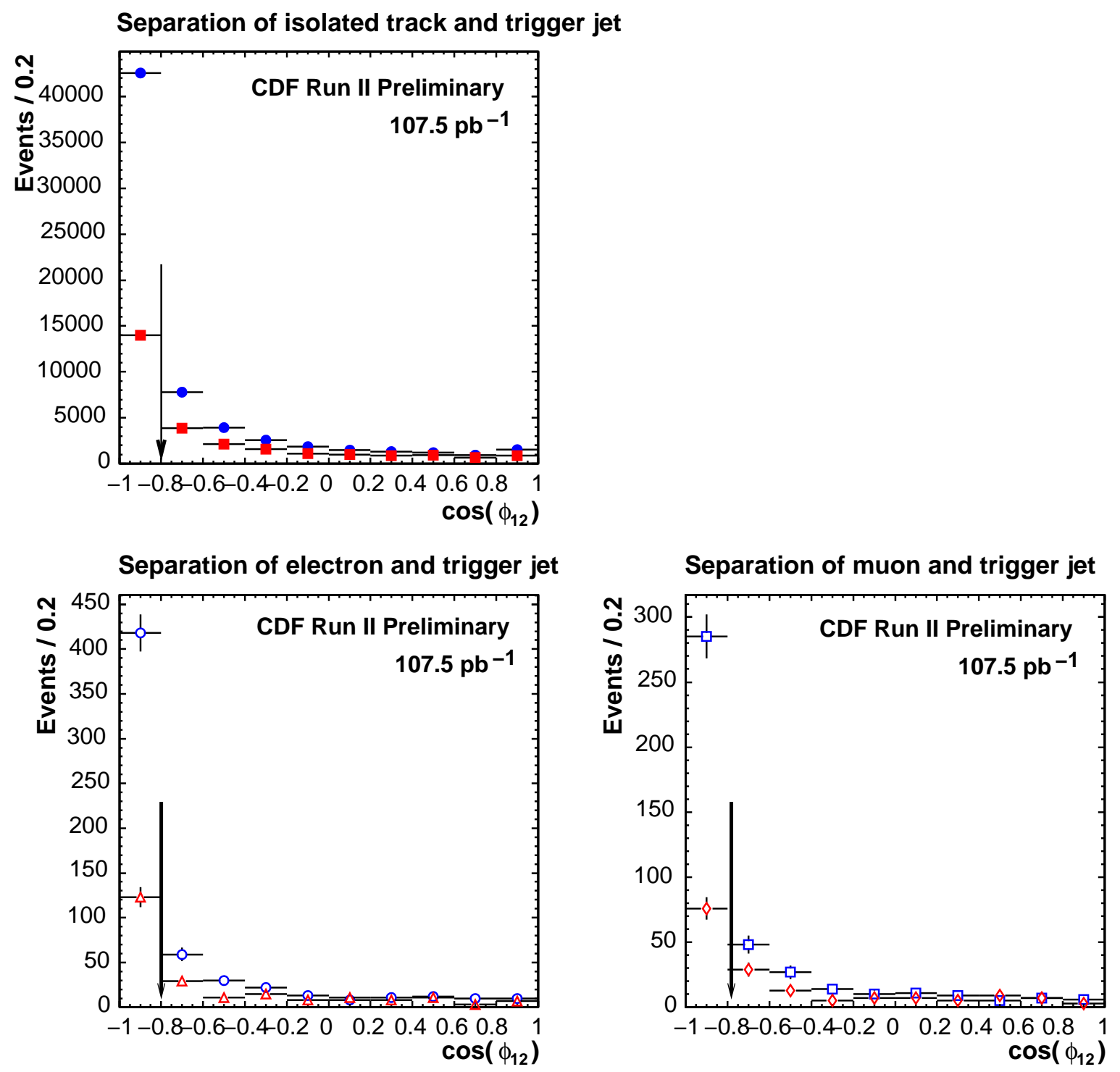

Figure 4.5: Upper plot is $\cos \phi_{12}$ distribution, where $\phi_{12}$ is $\phi$ separation of isolated track and trigger jet. The points are JET20 and JET50 samples. We define that $\cos \phi_{12}>$ -0.8 region is no-bias region and $\cos \phi_{12}<-0.8$ region is bias region. 

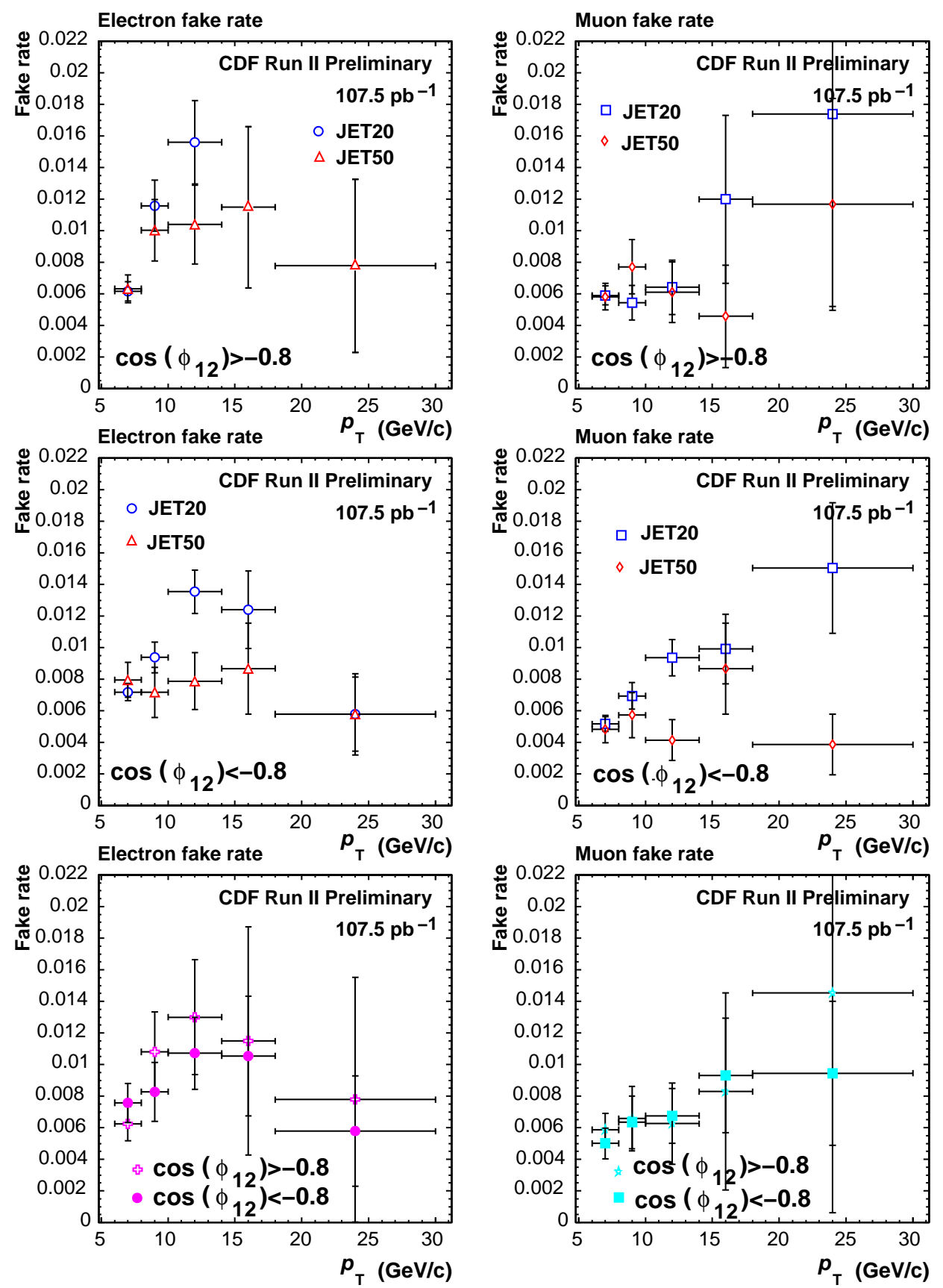

Figure 4.6: Electron and muon $p_{T}$ dependence of fake rate. Upper plots are fake rates in the no-bias region $\left(\cos \left(\phi_{12}\right)>-0.8\right)$. Middle plots are fake rates in the bias region $\left(\cos \left(\phi_{12}\right)<-0.8\right)$. Lower plots are combined fake rates for each region. We take the half of difference between no-bias fake rate and bias one as a systematic error. 

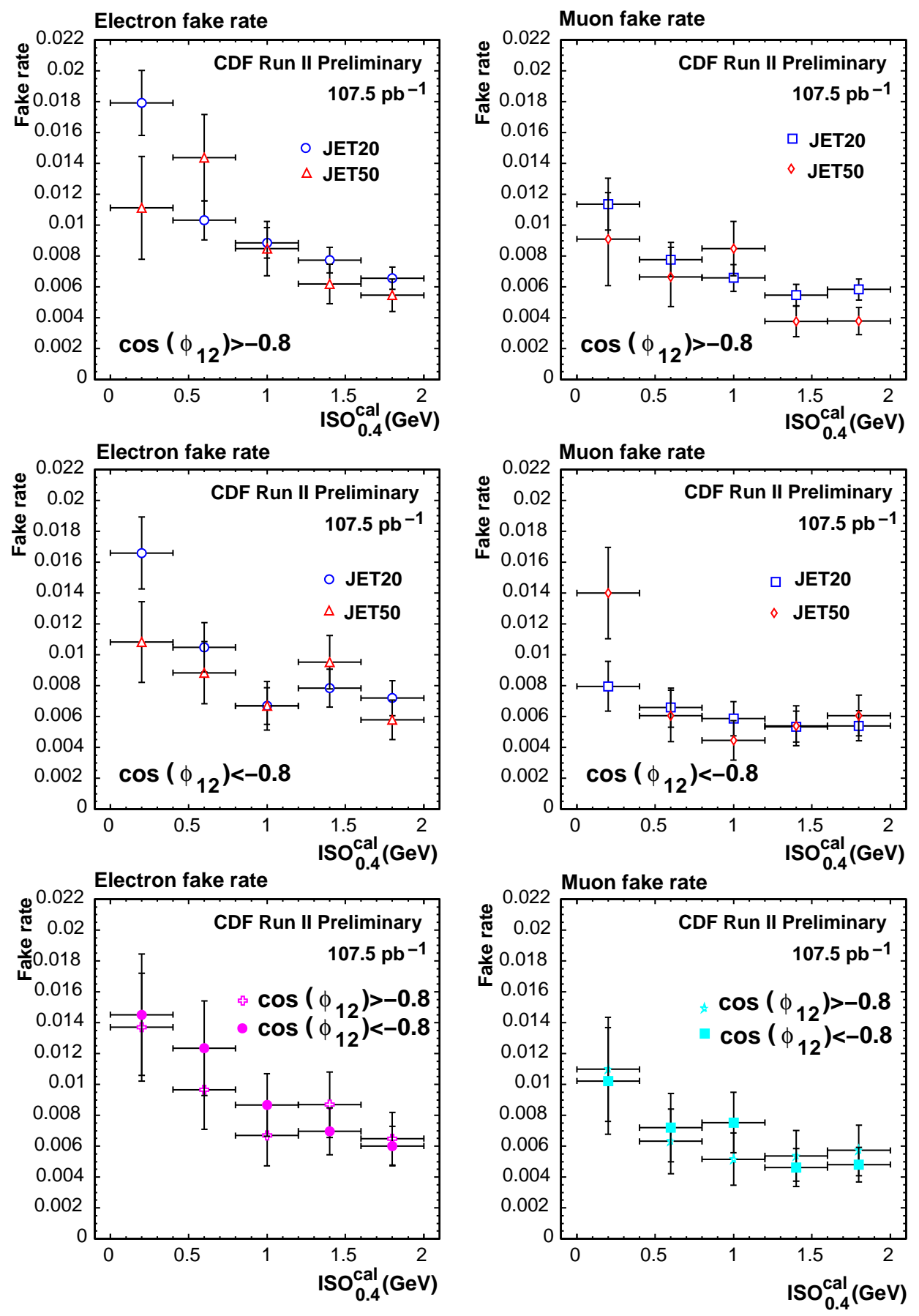

Figure 4.7: Electron and muon $I S O_{0.4}^{c a l}$ dependence of fake rate. Upper plots are fake rates in the no-bias region $\left(\cos \left(\phi_{12}\right)>-0.8\right)$. Middle plots are fake rates in the bias region $\left(\cos \left(\phi_{12}\right)<-0.8\right)$. Lower plots are combined fake rates for each region. We take the half of difference between no-bias fake rate and bias one as a systematic error. 

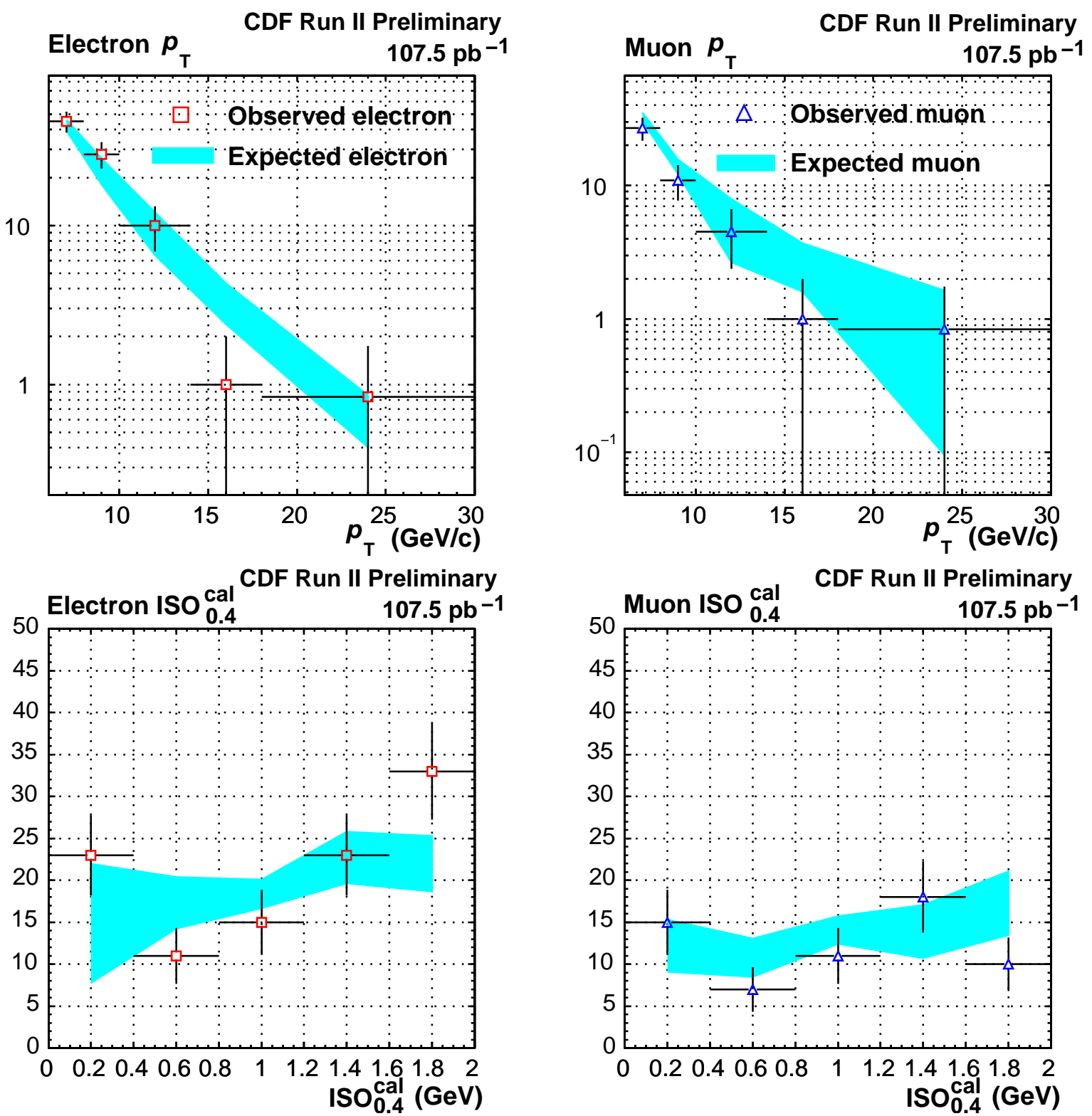

Figure 4.8: Expected and observed lepton $p_{T}$ and $I S O_{0.4}^{\text {cal }}$ distributions in the JET70 sample. The upper left (right) plot shows the electron (muon) $p_{T}$ distribution and the lower left (right) plot shows the $I S O_{0.4}^{\text {cal }}$ distribution. The filled regions represent the number of expected events with the estimated errors, and the points represent the number of observed events. 

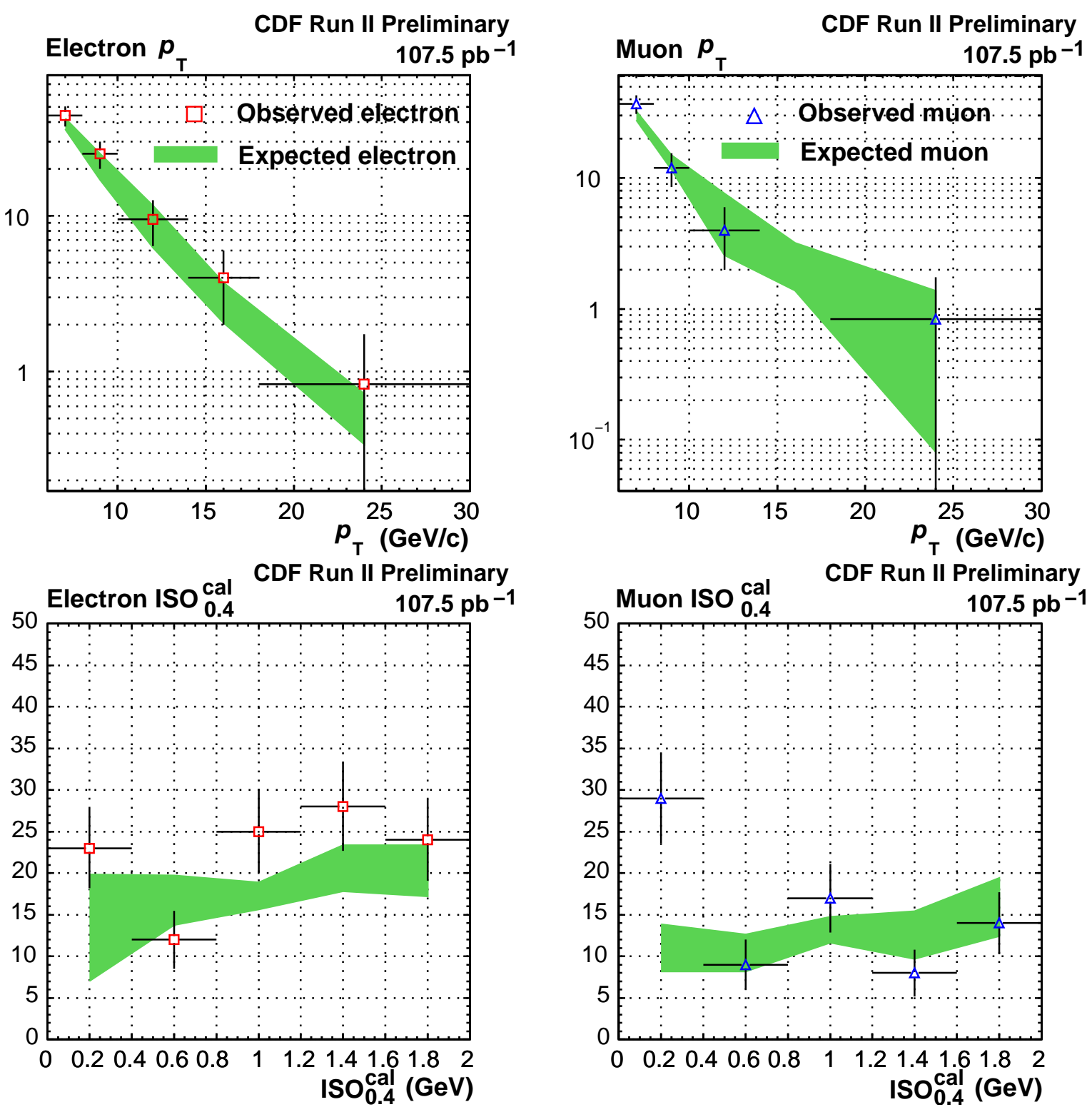

Figure 4.9: Expected and observed lepton $p_{T}$ and $I S O_{0.4}^{\text {cal }}$ distributions in the JET100 sample. The upper left (right) plot shows the electron (muon) $p_{T}$ distribution and the lower left (right) plot shows the $I S O_{0.4}^{c a l}$ distribution. The filled regions represent the number of expected events with the estimated errors, and the points represent the number of observed events. 

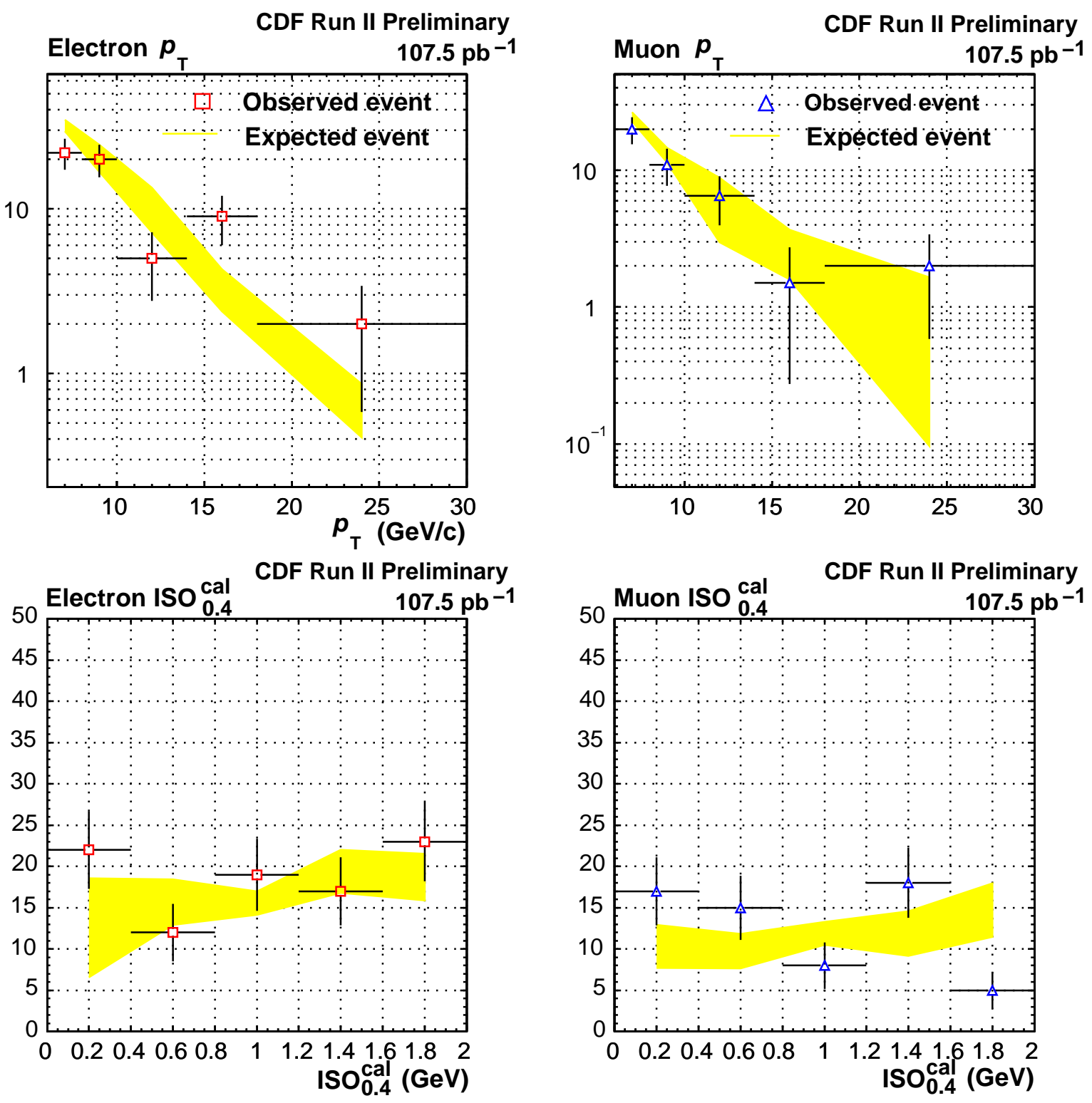

Figure 4.10: Expected and observed lepton $p_{T}$ and $I S O_{0.4}^{\text {cal }}$ distributions in the inclusive high- $E_{T}$ photon sample. The upper left (right) plot shows the electron (muon) $p_{T}$ distribution and the lower left (right) plot shows the $I S O_{0.4}^{c a l}$ distribution. The filled regions represent the number of expected events with the estimated error, and points represent the number of observed events. 


\subsection{Residual Photon Conversion Background}

A residual photon conversion is defined as a single electron event which is originated from the photon conversion with an unobserved partner track due to its low momentum. Figure 4.11 shows the theoretical calculation of the relative probability of energy sharing as a function of the fractional electron energy [76]. The higher probabilities of asymmetric energy sharing between an electron and a positron enhance the residual photon conversion events.

\subsubsection{Conversion Flag Rate}

The contribution of the residual photon conversion is included in the fake rate which we measured in the previous section. We need an assumption that the contribution of the residual photon conversion is the same between the JET samples and the like-sign dilepton events in the inclusive high- $p_{T}$ lepton samples in order to correctly reproduce the residual photon conversion background by using the fake rate. We compare the JET samples and the inclusive high- $p_{T}$ lepton samples by means of the conversion flag rate defined as follows:

$$
R_{\text {conv }}=\frac{\text { Number of conversions passing the electron identification cuts }}{\text { Number of electrons passing the electron identification cuts }},
$$

where the electron in the denominator is required to pass $E_{T}>6 \mathrm{GeV}, p_{T}>6 \mathrm{GeV} / c$ and survive the electron identification cuts described in the previous chapter. including the conversion veto, while the conversion event in the numerator is required to meet

the same conditions as the electron in the denominator except for the requirement of the conversion flag. For the JET samples we require the separation between the trigger jet and the isolated track in $\eta-\phi$ space to be $\Delta R>1.0$, and also we require $W$ veto $\left(\mathscr{E}_{T}<30 \mathrm{GeV}\right.$ and transverse mass $\left.<40 \mathrm{GeV}\right), Z$ veto, and cosmic veto in the same way as the fake rate measurement. For the inclusive high- $p_{T}$ lepton samples we require the like-sign dilepton selection cuts described in the previous chapter, and look at the 2nd leg of the like-sign dilepton pair. The conversion flag rate for each sample is shown in Figure 4.12. We calculate the combined conversion flag rates of the JET samples and the like-sign dilepton events in the inclusive high- $p_{T}$ lepton samples respectively as a weighted average. We obtain:

$$
\begin{gathered}
R_{\text {conv }}=0.26 \pm 0.10 \text { (Electron in JET samples), } \\
R_{\text {conv }}=1.23 \pm 0.26 \text { (Second leg electron in like-sign dilepton events). }
\end{gathered}
$$

We find the significant difference between the above two conversion flag rates. This is an evidence that the fraction of the residual photon conversion background is also 


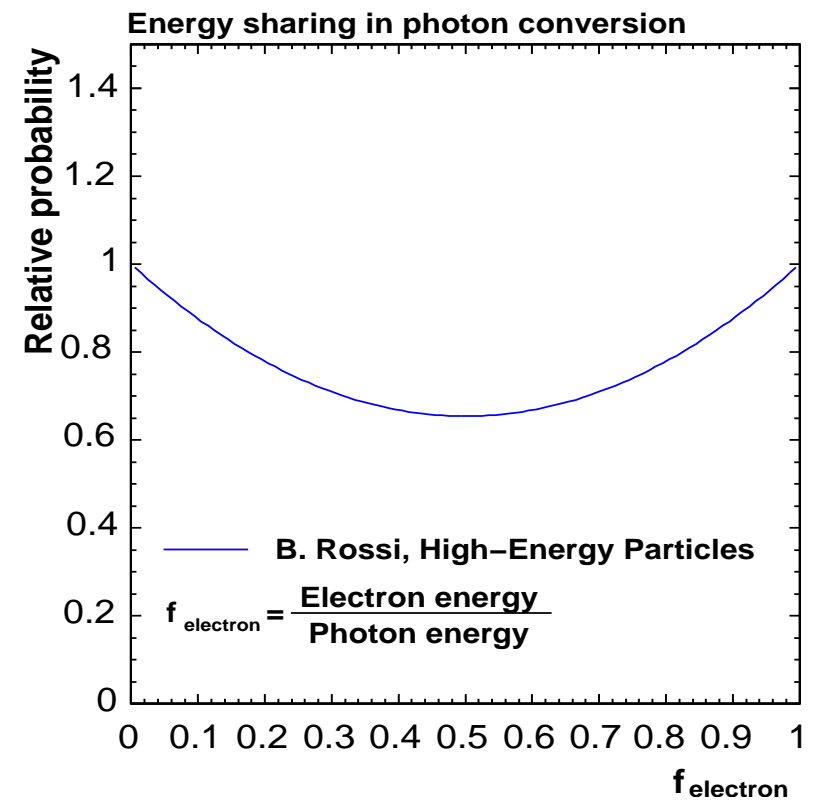

Figure 4.11: The theoretical calculation of the relative probability of energy sharing in the photon conversion as a function of the fractional electron energy [76].

different between the JET samples and the like-sign dilepton events in the inclusive high- $p_{T}$ lepton samples. We, hence, treat the residual photon conversions separately from the fake leptons. 


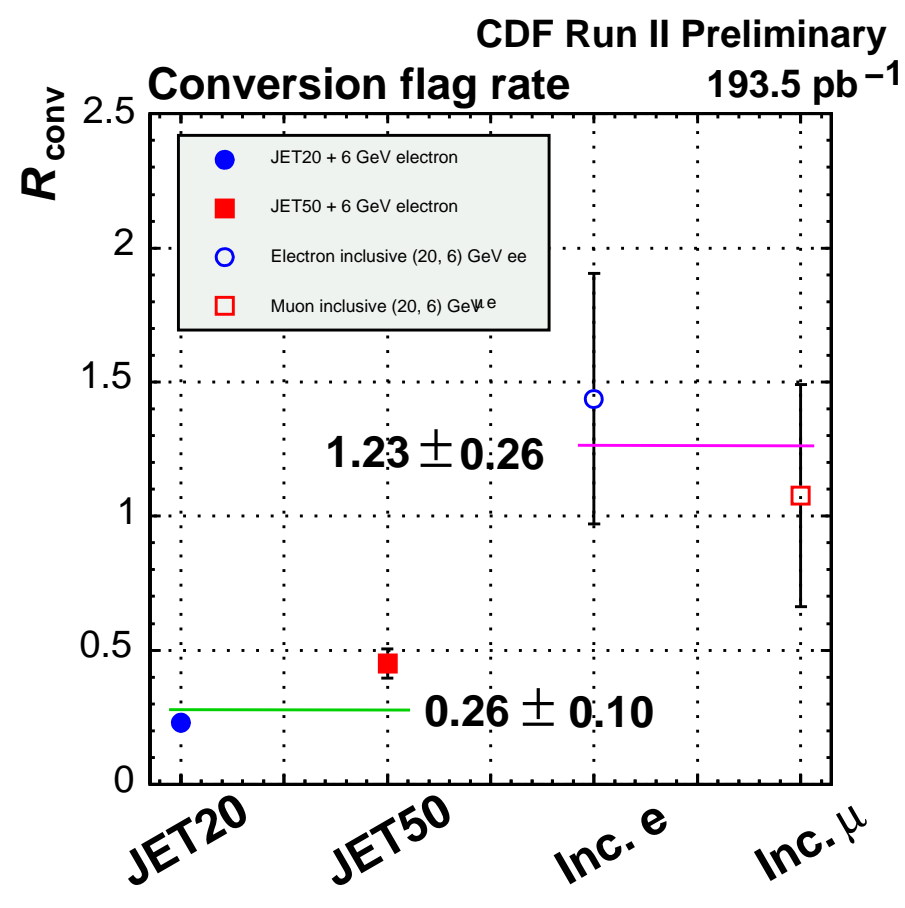

Figure 4.12: Conversion flag rate defined in Equation 4.2. A closed circle and a closed square correspond to the JET + electron $\left(E_{T}>6 \mathrm{GeV}\right)$ events in the JET20 and JET50 samples. An open circle and a open square correspond to the 2nd leg electrons $\left(E_{T}>6 \mathrm{GeV}\right)$ of the like-sign $e e$ or $\mu e$ events in the inclusive high- $p_{T}$ electron and muon samples. 


\subsubsection{Photon Conversion Tagging Efficiency}

We estimate the photon conversion tagging efficiency to obtain the contribution of the residual photon conversions to the like-sign dilepton events. Once we know the total tagging efficiency $\epsilon_{\text {tot }}$, we can calculate a ratio of the residual photon conversions to the tagged photon conversions $R_{\text {res }}$ as follows:

$$
\begin{gathered}
\epsilon_{\text {tot }}=\frac{N_{\text {tag }}}{N_{\text {IDele }}}=\epsilon_{\text {track }} \cdot \epsilon_{\text {cut }} \cdot \epsilon_{\mathrm{pt} 2.0}, \\
R_{\text {res }}=\frac{N_{\text {res }}}{N_{\text {tag }}}=\frac{N_{\text {IDele }}-N_{\text {tag }}}{N_{\text {tag }}}=\frac{1-\epsilon_{\text {tot }}}{\epsilon_{\text {tot }}},
\end{gathered}
$$

where

$$
\begin{array}{ll}
N_{\text {tag }}: & \text { Number of tagged conversions, } \\
N_{\text {IDele }}: & \text { Number of electrons passing the identification cuts } \\
& \text { excluding the conversion veto, } \\
N_{\text {res }} \quad: \text { Number of residual photon conversions. }
\end{array}
$$

If we obtain the $R_{\text {res }}$ ratio, we can estimate the amount of the residual conversions from that of the tagged conversions. We present the photon conversion tagging efficiency as a product of the partner track finding efficiency $\epsilon_{\text {track }}$, the conversion tagging cut efficiency $\epsilon_{\text {cut }}$, and the partner track finding efficiency above $2.0 \mathrm{GeV} / c, \epsilon_{\mathrm{pt2} 2.0}$. The detailed description and evaluation of these three efficiencies is given in the later subsections.

\subsubsection{Photon Conversion Monte Carlo Sample}

We use the Monte Carlo sample to obtain the kinematic information of the photon conversion. We here describes the photon conversion Monte Carlo sample. The prompt photons are generated by FAKE_EVENT module. We parameterize the photon $p_{T}$ spectrum as $p_{T}^{-\alpha}$, where $\alpha$ is set to be 6.9 in order to reproduce the observed $p_{T}$ spectrum of electrons originated from the photon conversion. The flat distribution of the photon density in $\eta$ and $\phi$ is used, and the photon conversion process is simulated by the cdfSim program. We use the $4 \mathrm{GeV}$ conversion electron samples for comparison. We demonstrate that our Monte Carlo sample reproduces the conversion points and the conversion energy sharing in Figure 4.13 and the $p_{T}$ spectrum of electrons in Figure 4.14. We require the electron identification cuts including the conversion tagging cuts for the Monte Carlo sample and data. We see that the Monte Carlo events reproduce the kinematics of photon conversion pairs reasonably well in the wide $p_{T}$ range. 

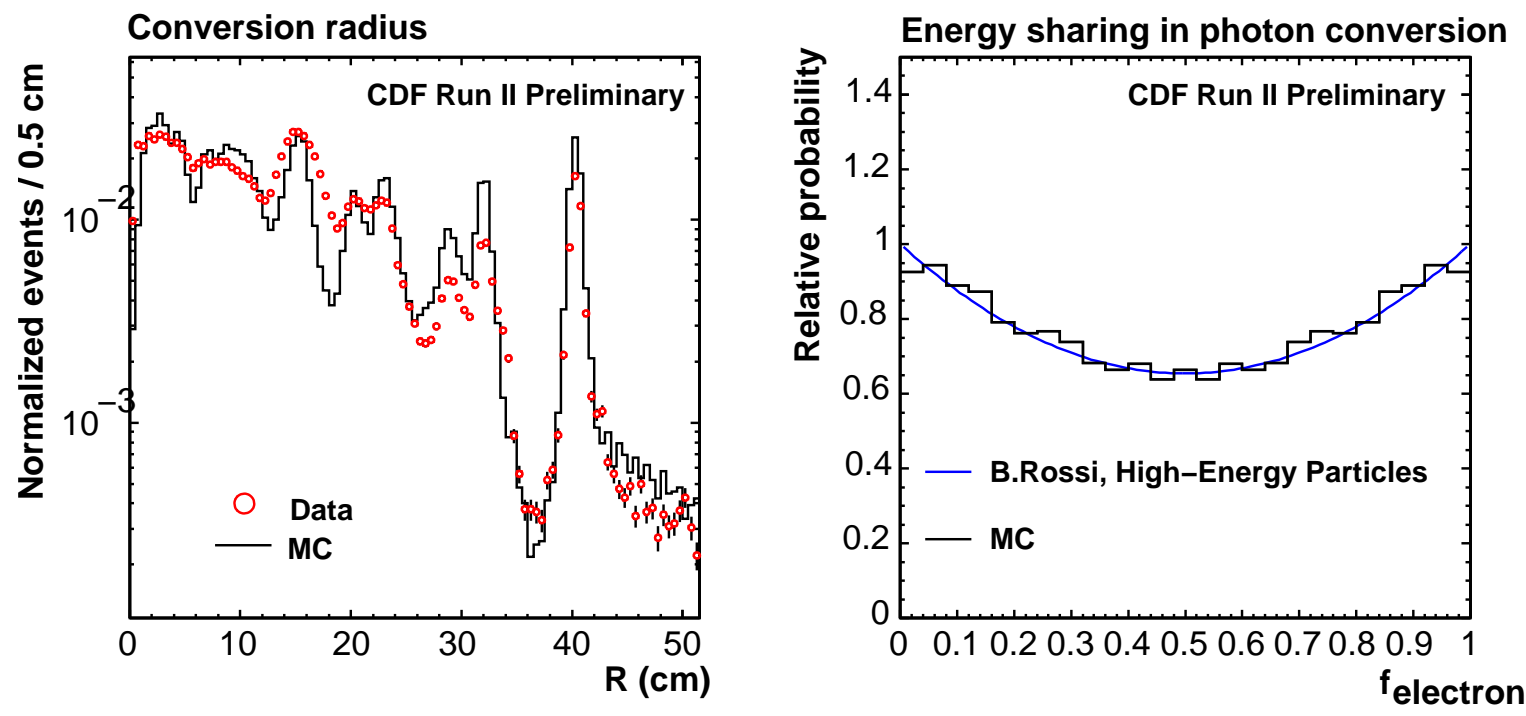

Figure 4.13: Distribution of the radius of the conversion points from the detector center (left), and the fractional electron energy (right). The points correspond to data and the histograms correspond to the Monte Carlo simulation.

\subsubsection{Partner Track Finding Efficiency}

First of all, we investigate the partner track finding efficiency of the photon conversion by comparing data with the Monte Carlo sample. Figure 4.15 shows the $p_{T}$ spectrum of the conversion partner tracks. We find that the generator level Monte Carlo spectrum has a strong peak at $p_{T}=0$, but the observed track spectrum does not. This means that the partner track finding efficiency decreases in the very low- $p_{T}$ region. This is thought to be mainly due to the low reconstruction efficiency for low- $p_{T}$ tracks, and we see the discrepancy reduces for the Monte Carlo spectra which are reconstructed after the detector simulation as shown in Figure 4.16. We estimate the $p_{T}$ dependence of the partner track finding efficiency by dividing the observed partner track distribution by the normalized Monte Carlo distribution. We normalize the Monte Carlo sample to data in the region above $2 \mathrm{GeV} / c$, where we see little discrepancy between data and the Monte Carlo sample as seen in Figure 4.16. The obtained partner track finding efficiency $\epsilon_{\text {track }}\left(p_{T}\right)$ is shown in Figure 4.17. The efficiency becomes flat in the region around $p_{T}$ of $2 \mathrm{GeV} / c$ and above. Note that since $\epsilon_{\text {track }}$ is a relative efficiency to this plateau region, we need to multiply the absolute efficiency of this region which is estimated in Section 4.3.6.

Although we have investigated the partner track finding efficiency as a function of the partner track $p_{T}$, we need to express the efficiency as a function of an observable of the conversion seed electrons in order to combine this efficiency with other elements as shown in Equation 4.5. Thus we redefine $\epsilon_{\text {track }}$ as a function of the conversion electron 

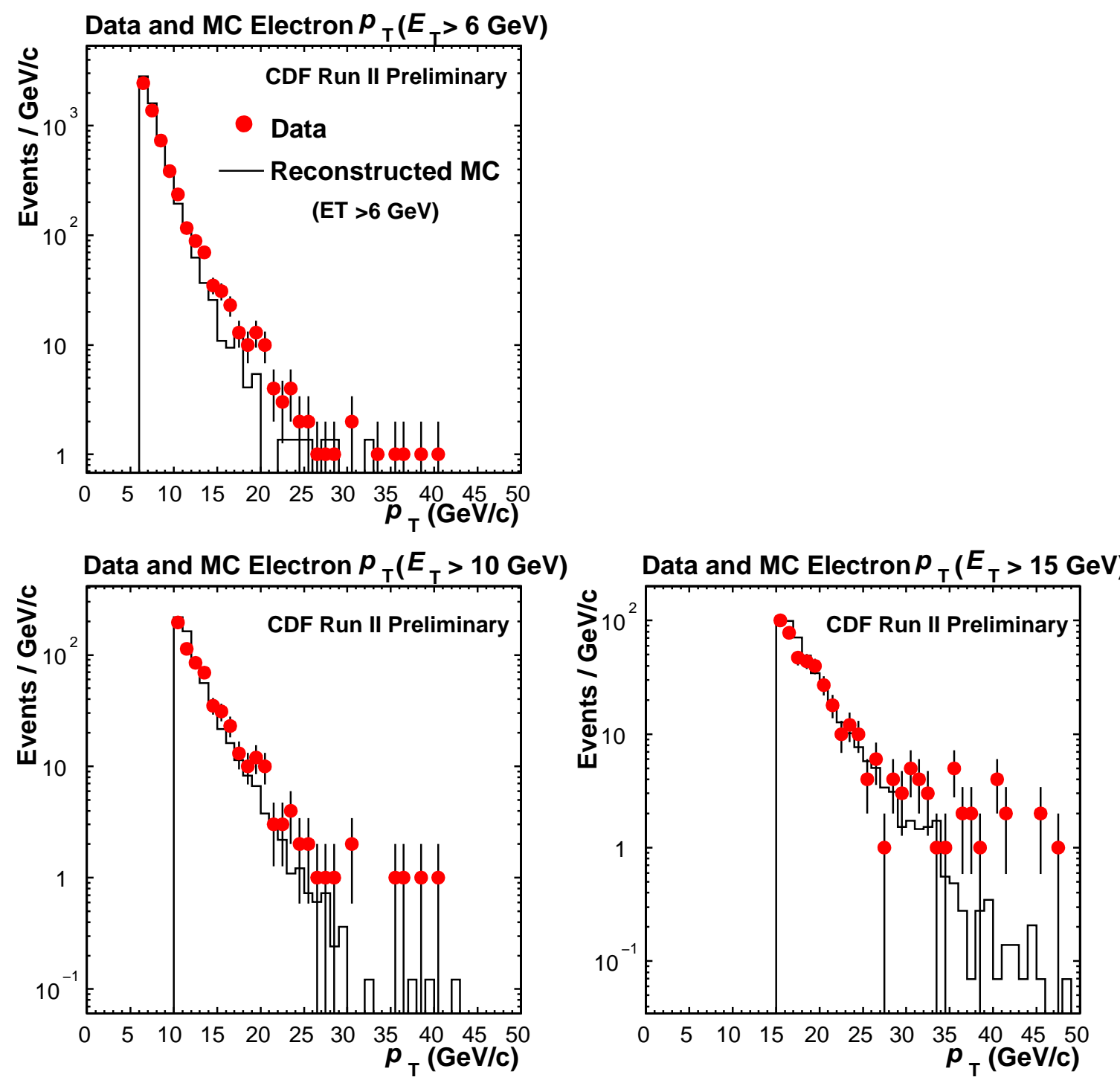

Figure 4.14: Conversion electron $p_{T}$ spectra for $E_{T}>6 \mathrm{GeV}$ (top left), $E_{T}>10 \mathrm{GeV}$ (bottom left), and $E_{T}>15 \mathrm{GeV}$ (bottom right). The points correspond to data and the histograms correspond to the reconstructed Monte Carlo simulation. The Monte Carlo sample is normalized to data in the region of the partner track $p_{T}>2 \mathrm{GeV} / c$. 

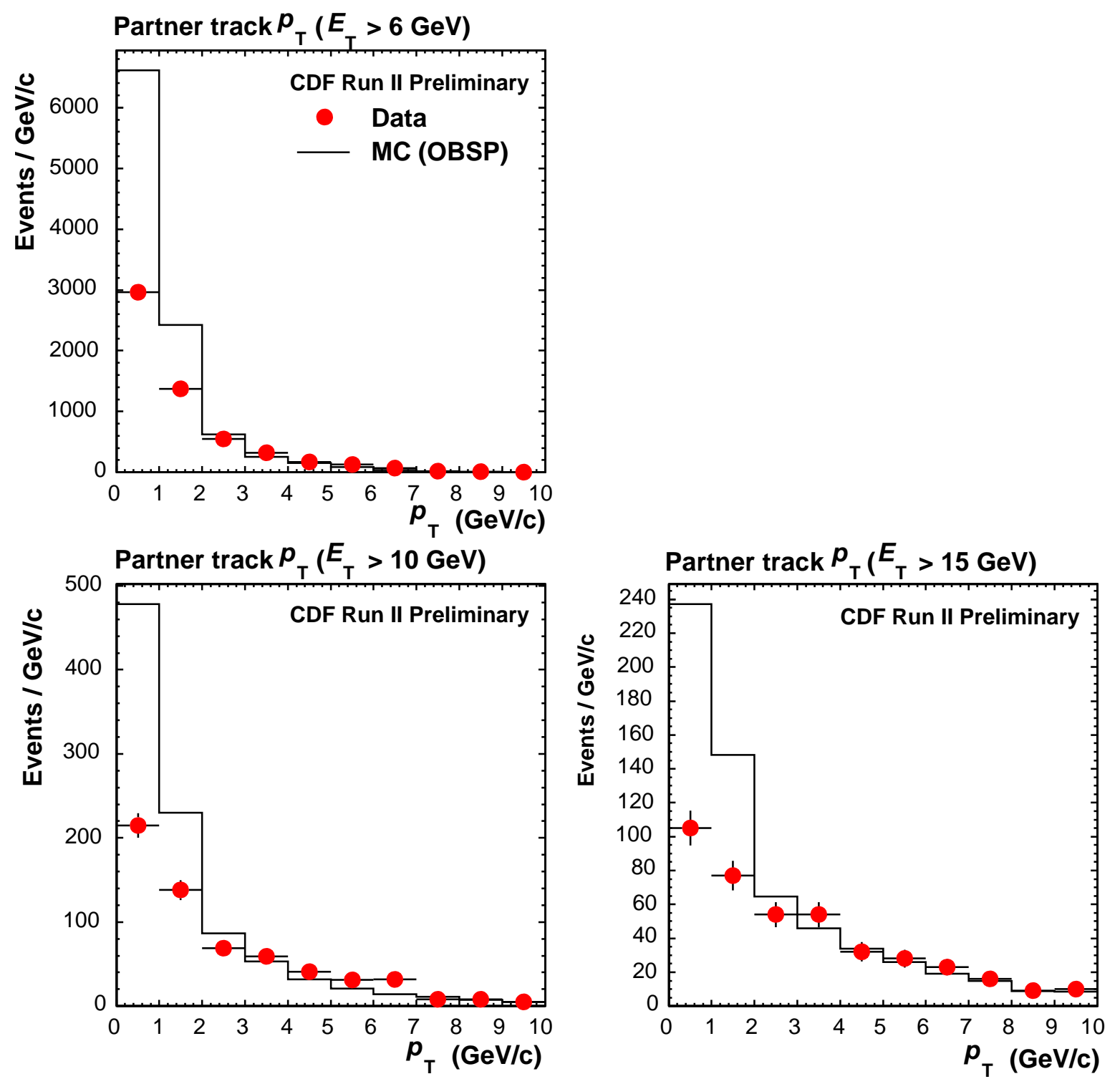

Figure 4.15: Conversion partner track $p_{T}$ spectra for $E_{T}>6 \mathrm{GeV}$ (top left), $E_{T}>10$ $\mathrm{GeV}$ (bottom left), and $E_{T}>15 \mathrm{GeV}$ (bottom right) of the seed electron. The points correspond to data and the histograms correspond to the $p_{T}$ distribution at the Monte Carlo OBSP level. The Monte Carlo sample is normalized to data in the region of the partner track $p_{T}>2 \mathrm{GeV} / c$. 

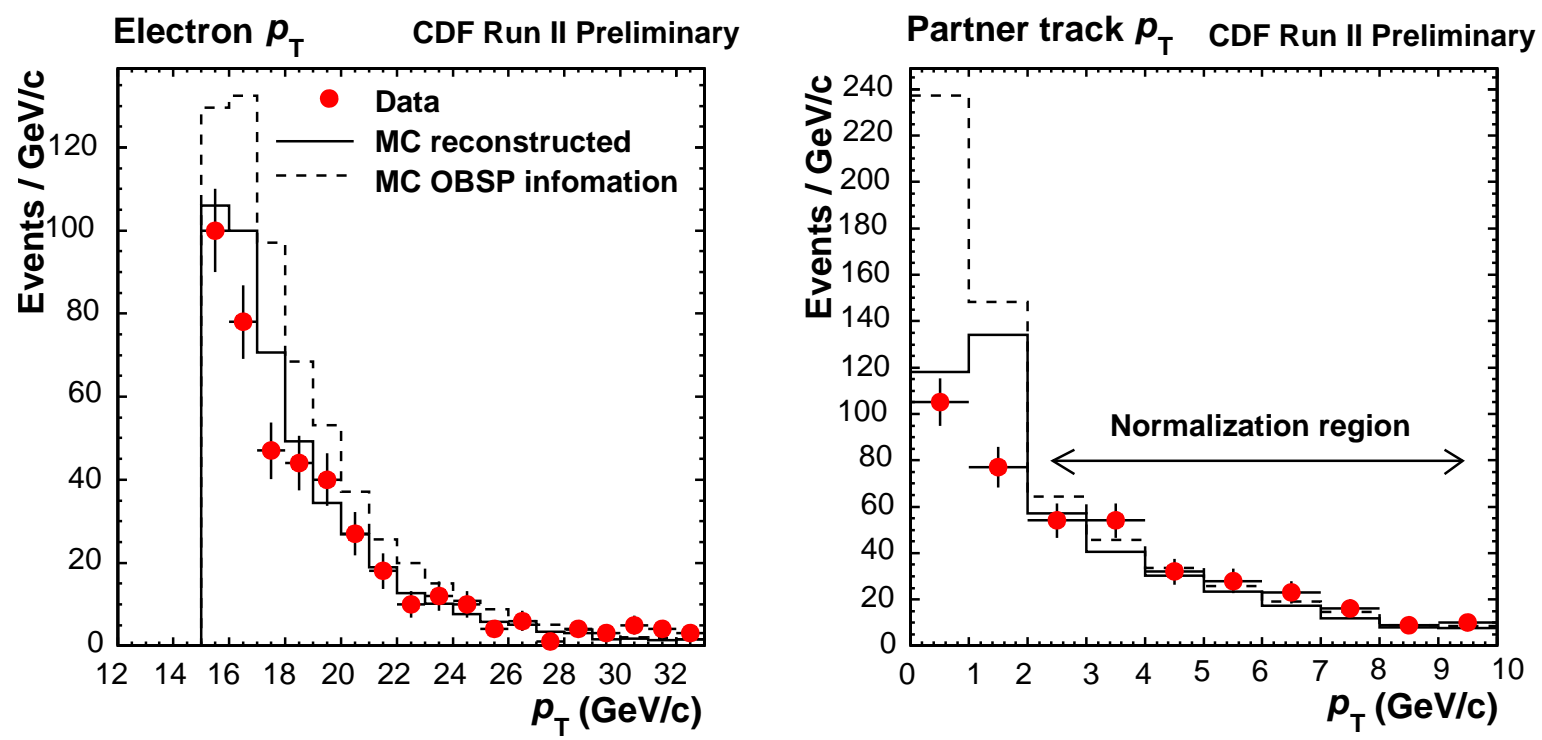

Figure 4.16: The conversion electron $p_{T}$ spectrum (left), and the partner track $p_{T}$ spectrum (right). The points correspond to data and the histograms correspond to the Monte Carlo simulation. The solid line histogram shows the spectrum which is reconstructed after the detector simulation, while the broken line histogram shows the spectrum at the OBSP level. The Monte Carlo spectra are normalized to data in the region of the partner track $p_{T}$ above $2 \mathrm{GeV} / c$.

CDF Run II Preliminary

\begin{tabular}{c|ccccc}
\hline \hline$E_{T}(\mathrm{GeV})$ & $6-8$ & $8-10$ & $10-14$ & $14-18$ & $18-30$ \\
\hline$\epsilon_{\text {track }}$ & $0.538 \pm 0.023$ & $0.567 \pm 0.021$ & $0.597 \pm 0.020$ & $0.601 \pm 0.029$ & $0.686 \pm 0.045$ \\
\hline \hline
\end{tabular}

Table 4.2: Partner track finding efficiencies for five $E_{T}$ regions of the conversion electrons.

$E_{T}$ as follows:

$$
\epsilon_{\text {track }}\left(E_{T}\right)=\int p_{T}^{\text {gen }} \cdot \epsilon_{\text {track }}\left(p_{T}\right) d p_{T} / \int p_{T}^{\text {gen }} d p_{T},
$$

where $p_{\mathrm{T}}^{\text {gen }}$ stands for the partner track $p_{T}$ at the generator level. We calculate the efficiency in the $E_{T}$ range from $6 \mathrm{GeV}$ to $30 \mathrm{GeV}$, which is presented in Table 4.2 and Figure 4.18. 


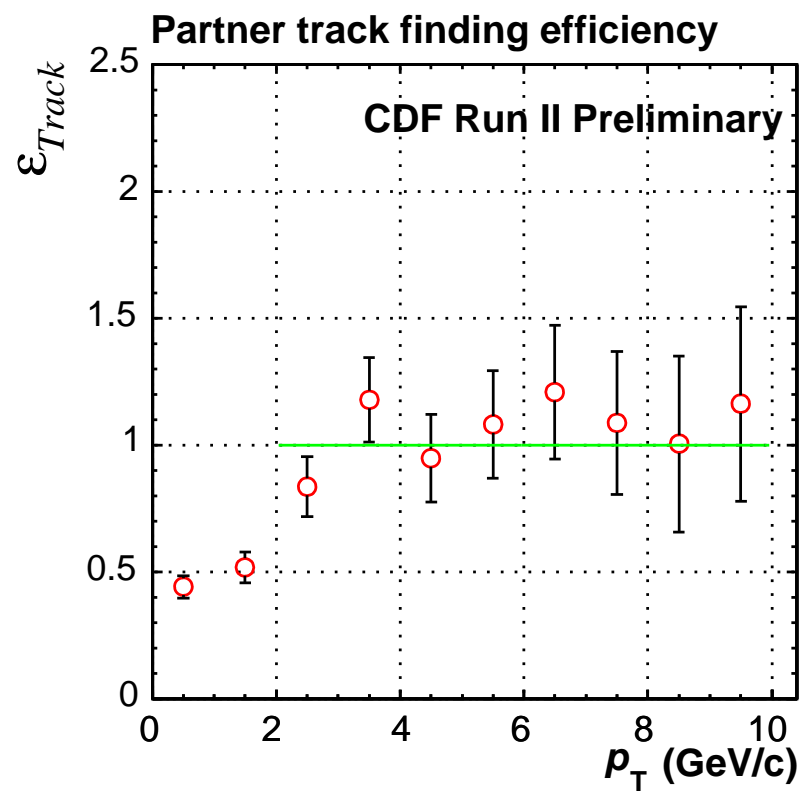

Figure 4.17: The partner track $p_{T}$ dependence of the partner track finding efficiency. We have normalized the Monte Carlo distribution to data in the region above $2 \mathrm{GeV} / c$ 


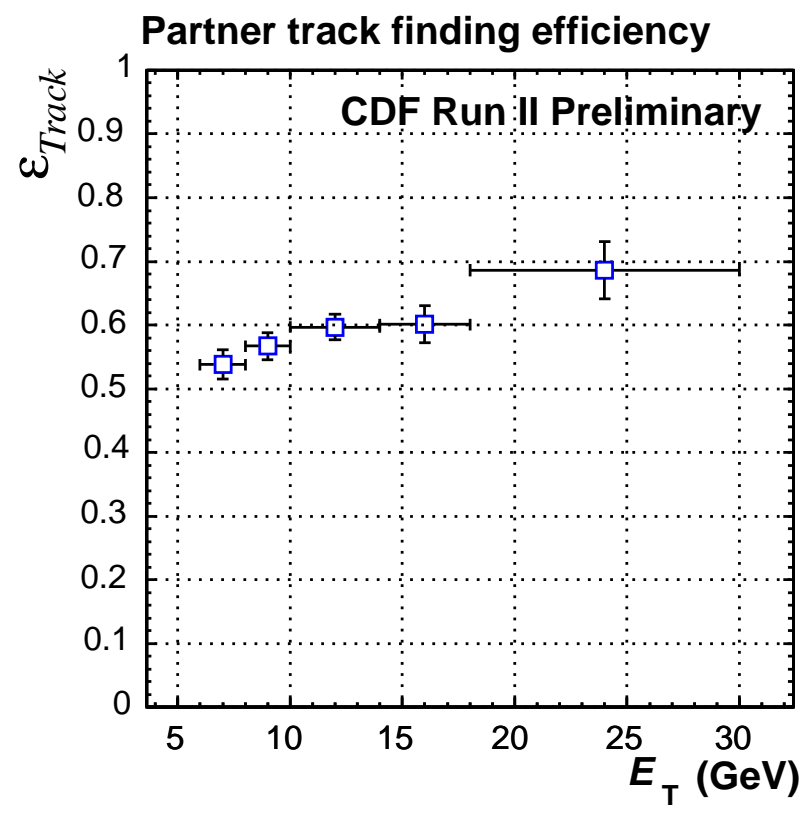

Figure 4.18: The conversion electron $E_{T}$ dependence of the partner track finding efficiency. We have normalized the Monte Carlo distribution to data in the region of the partner track $p_{T}$ above $2 \mathrm{GeV} / c$.

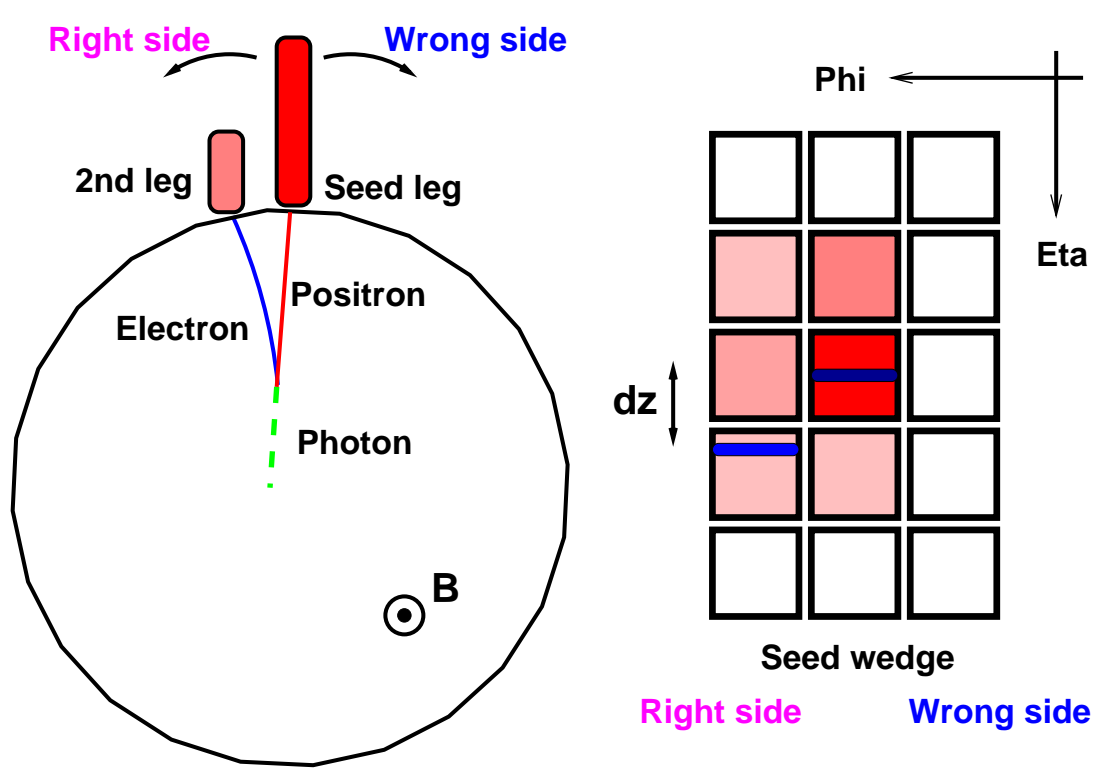

Figure 4.19: The illustrations of the conversion tagging by means of the CES strip hit information. 


\subsubsection{Conversion Tagging Cut Efficiency}

In the next step we estimate the efficiency of the conversion tagging cuts, of which contents are as follows:

$$
\left|\delta_{\mathrm{x} y}\right|<0.2 \mathrm{~cm} \text { and }|\Delta(\cot \theta)|<0.04
$$

where $\left|\delta_{x y}\right|$ is the distance of closest approach between a pair of tracks of the conversion candidates. Since we need a conversion sample which is independent of the above tagging criteria, we use the hit information of the CES strips to tag the photon conversion. This is basically the same manner that was studied in [72]. A distinct feature of the photon conversion pair is that the electron and the positron have approximately the same $z$ position at any radius. When the electron and the positron separate enough so that they reach different $\phi$ wedges as illustrated in Figure 4.19, we can measure each $z$ position of the electron and the positron on the CES plane. We look for the highest energy CES cluster pointing to the wedge next to the seed electron. The CES cluster has to be placed in the "right" side $\phi$ wedge as expected from the charge configuration. We demonstrate that the $z$ position difference $d z$ has a peak around zero in the right side $\phi$ and does not in the wrong side in Figure 4.20. We look into the $8 \mathrm{GeV}$ inclusive electron data and the conversion Monte Carlo sample for the consistency check. We simply look for the electron which passes $E_{T}>8 \mathrm{GeV}$ and the electron identification cuts, then look for the highest energy CES cluster in the nearest three towers for each $\phi$ side. We use the "unbiased" CES cluster collection which is created from a list of wires or strip seeds over a threshold energy (150 MeV).

We use the above conversion candidates to measure the tagging cut efficiency. In order to ensure that a conversion electron-positron pair exists in the event, we require the following conditions of the electron which passes the identification cuts excluding the conversion veto:

- The extrapolation of the oppositely charged track points to the tower containing the highest energy CES strip cluster at the "right" side $\phi$ wedge,

- $E_{\mathrm{ces}} / p>0.5$ and CEM $E / p>0.7$.

The $d z$ distributions of the events which pass and fail the conversion tagging cuts presented in Equation 4.8 are shown in Figure 4.21. We evaluate the number of passed and failed events by counting the number of events over the flat distribution of background for each $d z$ distribution. Then we obtain the efficiency $\epsilon_{\text {cut }}$ by calculating a ratio of the number of passed events to that of total events excluding the background. Table 4.3 lists the efficiencies calculated for several cuts on the electron $E_{T}$. By taking an average as the combined value and a standard deviation as the systematic error, we obtain the conversion tagging efficiency $\epsilon_{\text {cut }}$ to be: 

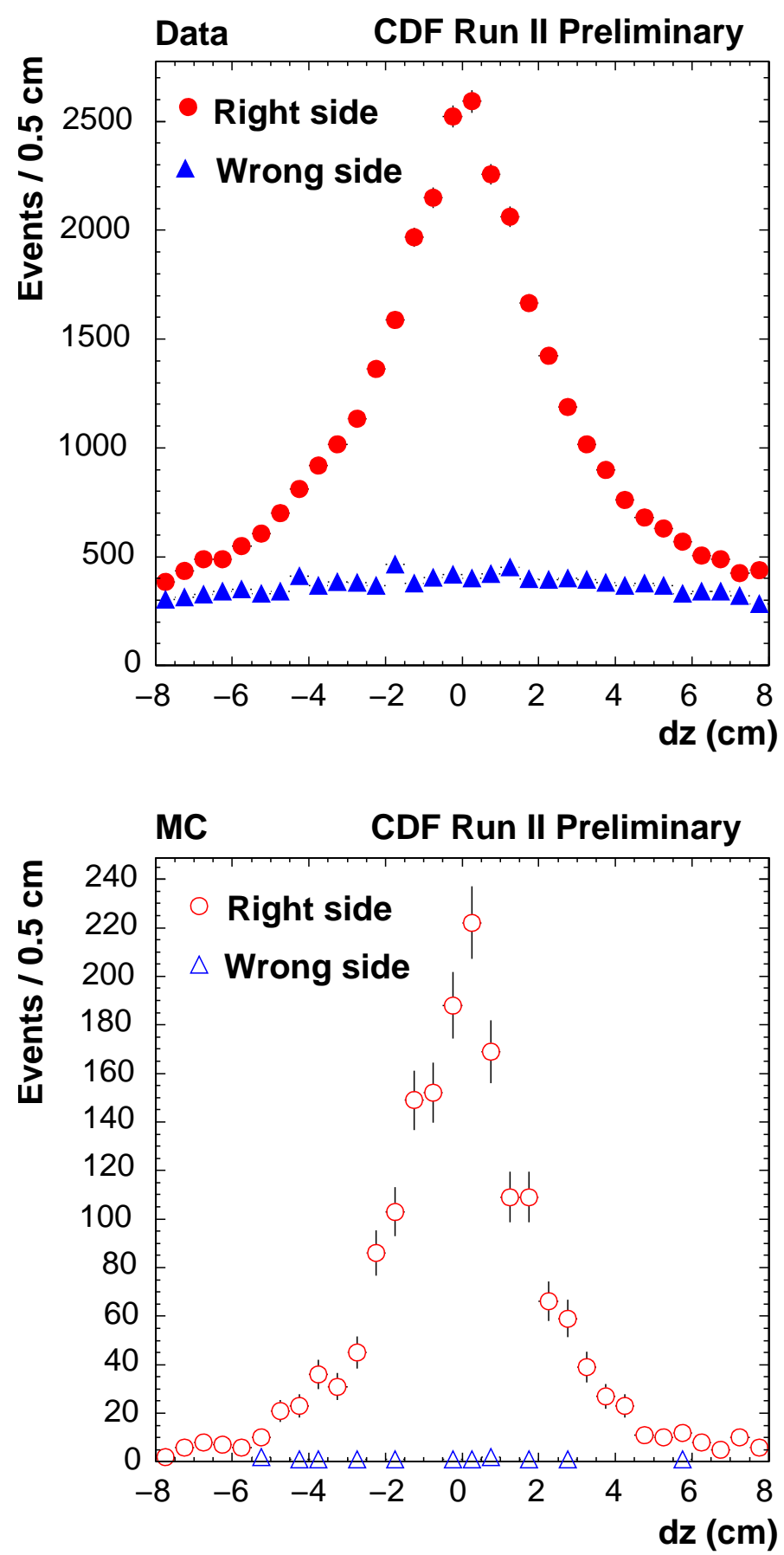

Figure 4.20: The $z$ position difference between the electron passing the identification cuts and the highest energy CES cluster in the nearest three towers for each $\phi$ side (right side and wrong side). The upper plot shows data and the lower plot shows the Monte Carlo simulation. 

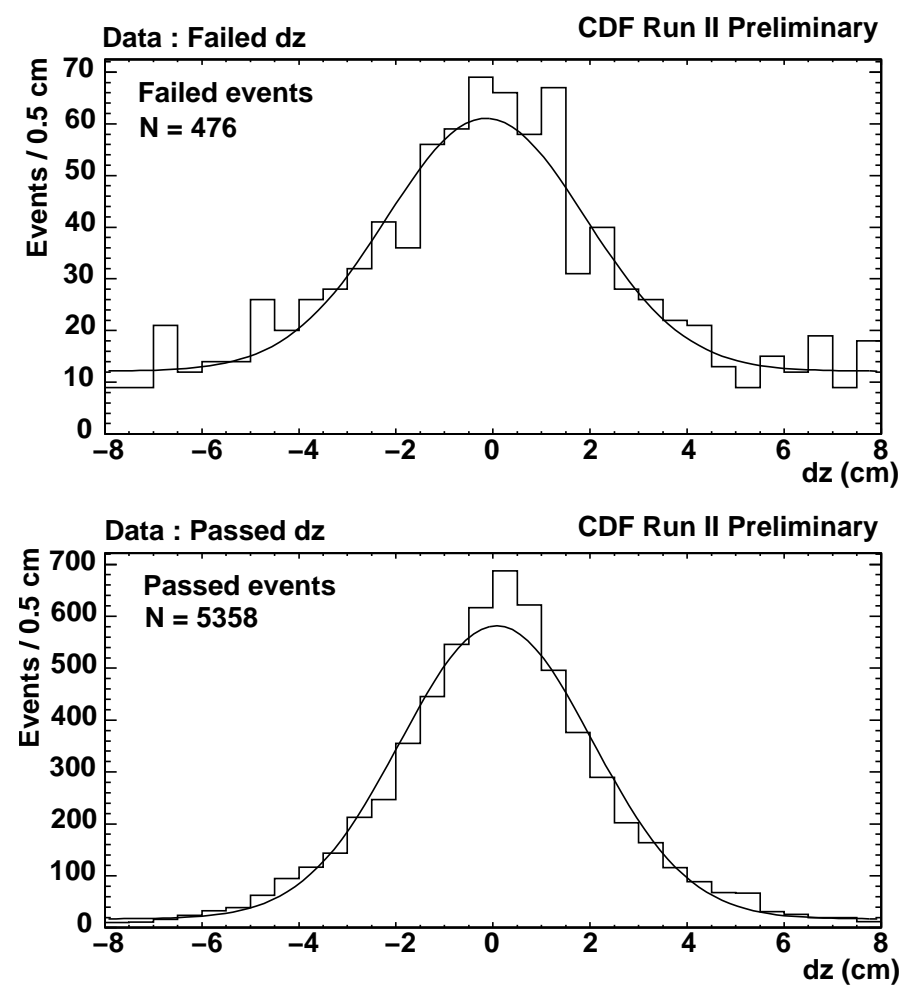

Figure 4.21: The difference of the electron-positron $z$ coordinates on the CES plane for the pairs passing and failing the conversion tagging cuts. The electron $E_{T}$ cut is applied at $8 \mathrm{GeV}$.

\begin{tabular}{c|cccc}
\hline & & & \multicolumn{2}{c}{ CDF Run II Preliminary } \\
\hline \hline$E_{T}(\mathrm{GeV})$ & $>8$ & $>10$ & $>14$ & $>18$ \\
\hline$\epsilon_{\text {cut }}$ & $0.918 \pm 0.003$ & $0.914 \pm 0.004$ & $0.926 \pm 0.008$ & $0.927 \pm 0.010$ \\
\hline \hline
\end{tabular}

Table 4.3: Conversion tagging cut efficiencies for four $E_{T}$ cuts of the conversion electrons.

$$
\epsilon_{\text {cut }}=0.921 \pm 0.006 \text { (stat) } \pm 0.006 \text { (syst) }
$$




\subsubsection{Partner Track Finding Efficiency above $2 \mathrm{GeV} / c$}

The partner track finding efficiency $\epsilon_{\text {track }}$ was obtained in Section 4.3.4. We normalized the Monte Carlo sample to data in the region above $2 \mathrm{GeV} / c$ because we found the efficiency curve became flat above $2 \mathrm{GeV} / c$. Since $\epsilon_{\text {track }}$ is a relative efficiency to this plateau region, we now estimate the absolute value of the track finding efficiency in this region, $\epsilon_{\mathrm{pt} 2}$. Since we cannot use the COT track based samples to estimate the track finding efficiency, we use the same method that is described in the previous subsection to extract the photon conversion samples. We collect the samples having CES strip clusters in the nearest three towers from the seed electron at the "right" side $\phi$ wedge. In order to ensure the partner track $p_{T}$ to be greater than $2 \mathrm{GeV} / c$, we take the following procedures:

- Assume the existence of a track associated to the highest energy CES strip cluster in the nearest three towers at the right side $\phi$ wedge,

- Assume the associated track $p_{T}$ to be $2 \mathrm{GeV} / c$ and calculate the track momentum $p$ from the CES strip z position or $\eta$,

- Require $E_{\text {ces }} / p>0.5$ and CEM $E / p>0.7$.

See [72] for more detail on the above method. Then we look for the oppositely charged track which is extrapolated to the tower including the above-mentioned highest energy CES strip cluster. Figure 4.22 shows the $d z$ distributions for cases of "track found" and "no track found". We calculate the efficiency $\epsilon_{\mathrm{pt} 2}$ from the inclusive $8 \mathrm{GeV}$ electron data and inclusive high- $p_{T}$ electrons. The obtained efficiencies are listed in Table 4.4 for several cuts on the conversion electron $E_{T}$. We take an average as the combine value and a standard deviation as the systematic error. We obtain:

$$
\epsilon_{\mathrm{pt} 2}=0.983 \pm 0.006 \text { (stat) } \pm 0.015 \text { (syst) }
$$

CDF Run II Preliminary

\begin{tabular}{c|cccc}
\hline \hline$E_{T}(\mathrm{GeV})$ & $>8$ & $>10$ & $>14$ & $>18$ \\
\hline$\epsilon_{\mathrm{pt} 2}$ & $0.967 \pm 0.005$ & $0.984 \pm 0.006$ & $0.996 \pm 0.008$ & $0.997 \pm 0.006$ \\
\hline \hline
\end{tabular}

Table 4.4: Partner track finding efficiencies above $2 \mathrm{GeV} / c$ for four $E_{T}$ cuts of the conversion electrons. 

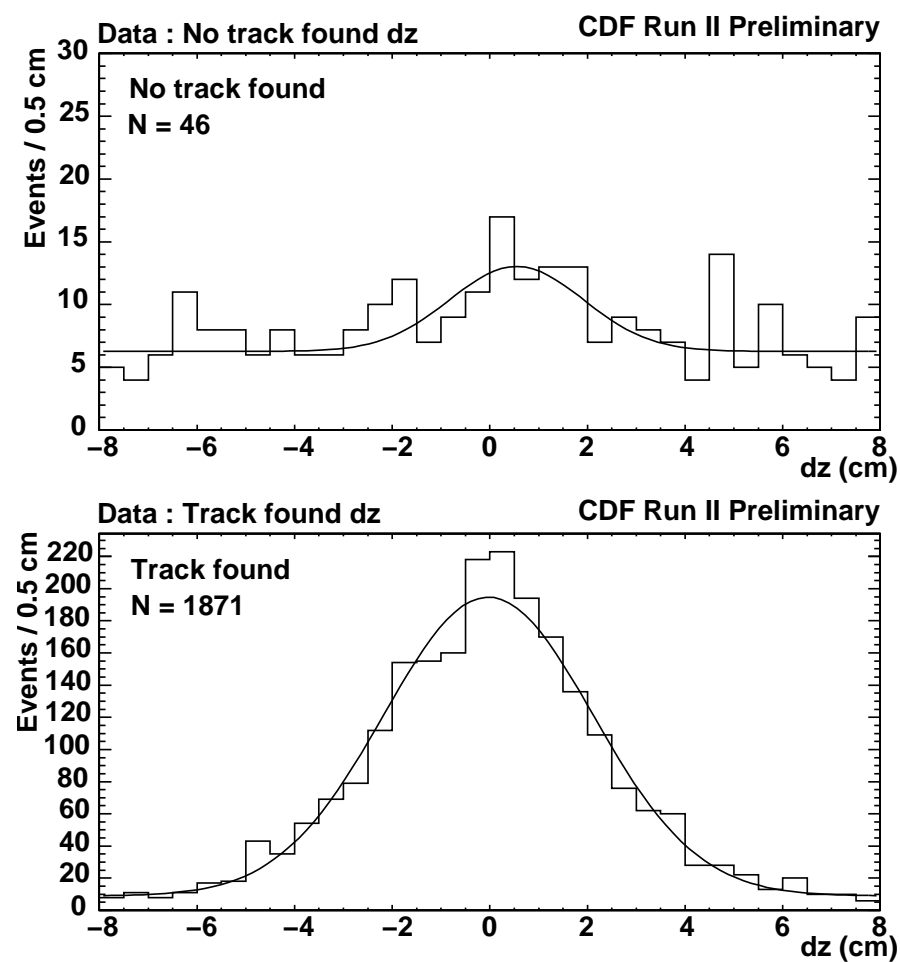

Figure 4.22: The difference of the electron-positron $z$ coordinates on the CES plane for cases of "no track found" and "track found". The electron $E_{T}$ cut is applied at 8 $\mathrm{GeV}$. 


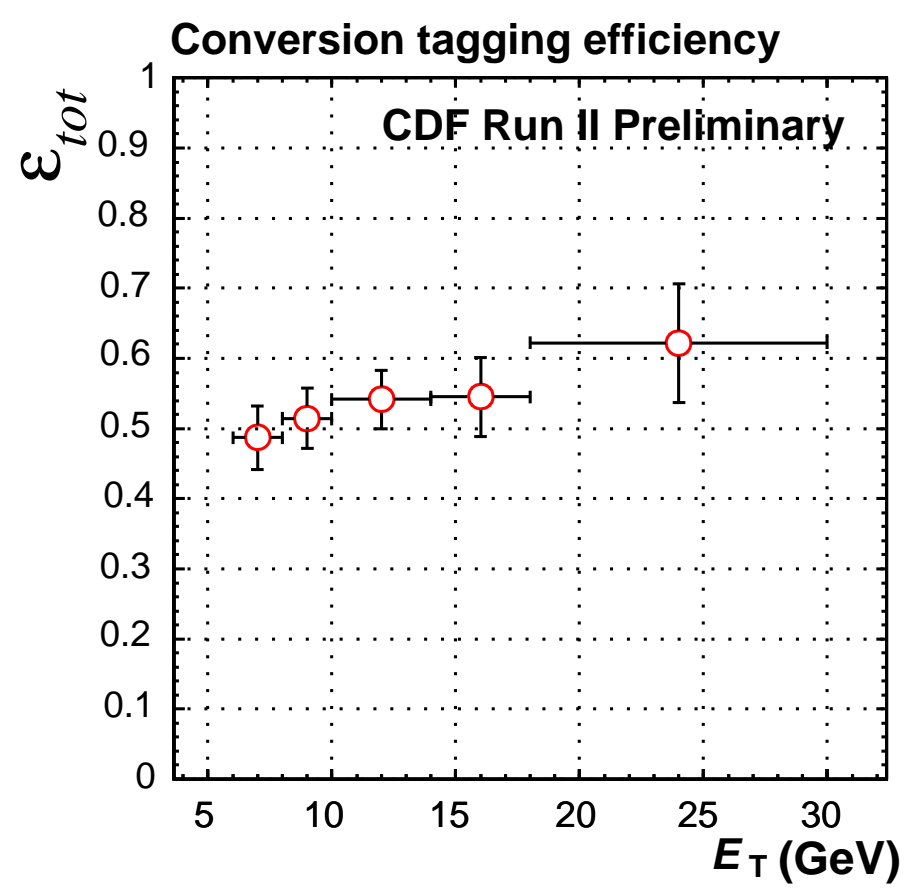

Figure 4.23: The electron $E_{T}$ dependence of the photon conversion tagging efficiency.

\begin{tabular}{c|ccccc}
\multicolumn{1}{c}{} & & & \multicolumn{2}{c}{ CDF Run II Preliminary } \\
\hline \hline$E_{T}(\mathrm{GeV})$ & $6-8$ & $8-10$ & $10-14$ & $14-18$ & $18-30$ \\
\hline$\epsilon_{\text {tot }}$ & $0.487 \pm 0.046$ & $0.513 \pm 0.043$ & $0.540 \pm 0.042$ & $0.544 \pm 0.057$ & $0.621 \pm 0.085$ \\
\hline \hline
\end{tabular}

Table 4.5: Overall conversion tagging efficiencies for five $E_{T}$ regions of the conversion electrons.

\subsubsection{Overall Efficiency and Residual Conversion Ratio}

We calculate the overall conversion tagging efficiency $\epsilon_{\text {tot }}$ defined in Equation 4.5. The obtained results are shown in Table 4.5 and Figure 4.23. We see that $\epsilon_{\text {tot }}$ increases from $50 \%$ to $60 \%$ as the conversion electron $E_{T}$ increases. Once we get the overall conversion tagging efficiency, we can calculate the residual conversion ratio $R_{\text {res }}$, which is defined in Equation 6. Table 4.6 and Figure 4.24 show the $R_{\text {res }}$ ratio as a function of the conversion electron $E_{T}$. We will use this ratio to estimate the lepton plus residual conversion background in Section 4.5. 


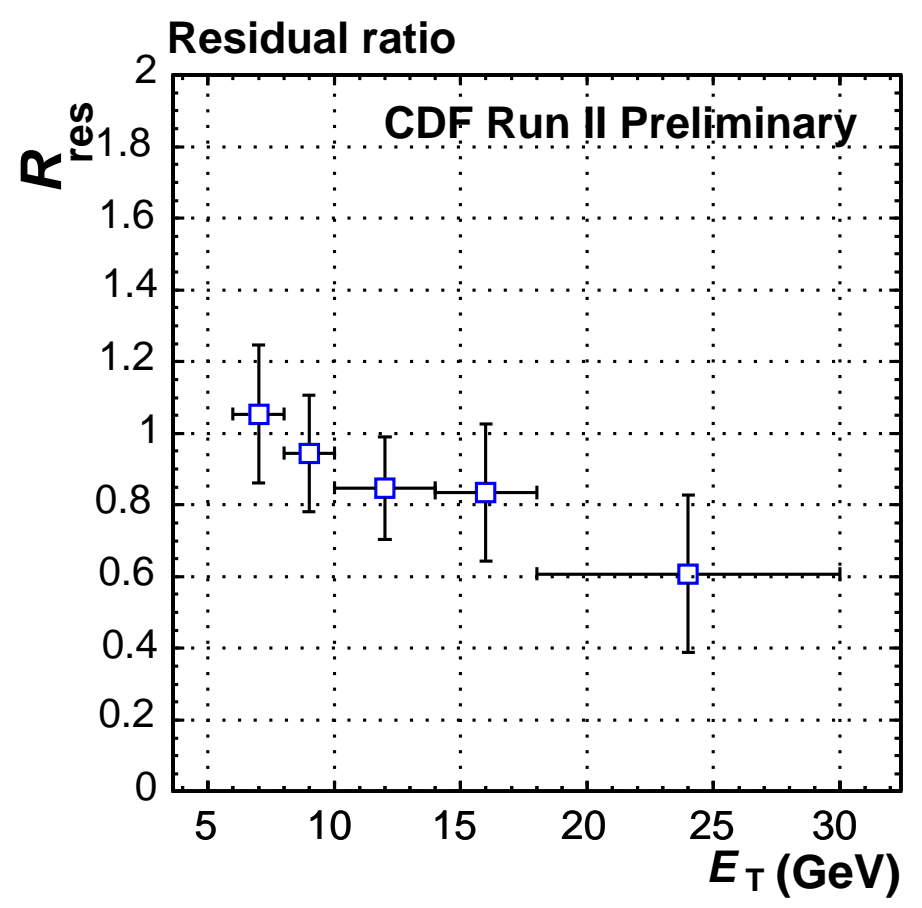

Figure 4.24: The electron $E_{T}$ dependence of the residual photon conversion ratio.

\begin{tabular}{c|ccccc}
\hline \hline$E_{T}(\mathrm{GeV})$ & $6-8$ & $8-10$ & $10-14$ & \multicolumn{3}{c}{ CDF Run II Preliminary } \\
\hline$R_{\text {res }}$ & $1.05 \pm 0.19$ & $0.948 \pm 0.163$ & $0.856 \pm 0.143$ & $0.838 \pm 0.191$ & $0.610 \pm 0.220$ \\
\hline \hline
\end{tabular}

Table 4.6: Residual photon conversion ratios for five $E_{T}$ regions of the conversion electrons. 


\subsection{Corrected Electron Fake Rate}

In Section 4.3.1 we saw significant difference of the conversion flag rate between the JET and inclusive high- $p_{T}$ lepton samples. This means that the contribution of the residual photon conversions is also different for each sample, and we hence needed the separate consideration of the residual conversions from the fake leptons. In order to avoid double counting of the residual conversion, we make corrections in the original electron fake rate in this section.

We calculate the corrected electron fake rate $R_{\text {fake }}^{\text {corr }}$ as follows:

$$
R_{\text {fake }}^{\text {corr }}=\frac{N_{\text {fake }}}{N_{\text {iso }}}=\left(1-R_{\text {conv }} \cdot R_{\text {res }}\right) \cdot R_{\text {fake }},
$$

where $R_{\text {conv }}$ is the conversion flag rate, $R_{\text {res }}$ is the residual photon conversion ratio, and $R_{\text {fake }}$ is the original electron fake rate. We define the three variables by the following relations.

$$
\begin{gathered}
R_{\text {conv }}=\frac{N_{\text {conv }}}{N_{\text {IDele }}}, \quad R_{\text {res }}=\frac{N_{\text {res }}}{N_{\text {conv }}}, \quad R_{\text {fake }}=\frac{N_{\text {IDele }}}{N_{\text {iso }}}, \\
N_{\text {IDele }}=N_{\text {fake }}+N_{\text {res }}
\end{gathered}
$$

where $N_{\text {fake }}$ is number of fake electrons except residual photon conversion in JET sample, $N_{\text {conv }}$ is number of tagged photon conversions, $N_{\text {IDele }}$ is number of electrons which is passed identification cuts in JET sample, $N_{\text {res }}$ is number of residual photon conversions, and $N_{\text {iso }}$ is number of isolated track in JET sample.

Since we measured $R_{\text {fake }}$ as a function of the track $p_{T}$ and also as a function of $I S O_{0.4}^{c a l}$, we check whether $R_{\text {conv }}$ and $R_{\text {res }}$ also have dependences on the track $p_{T}$ and

$I S O_{0.4}^{c a l}$. The results on $R_{\text {conv }}$ are shown in Figure 4.25 for the JET samples (JET20 and JET50). We observe the dependence of $R_{\text {conv }}$ on $p_{T}$, but not on $I S O_{0.4}^{\text {cal }}$. As for the residual conversion ratio $R_{\text {res }}$, we measured it as a function of the electron $E_{T}$. In order to obtain the dependence on the track $p_{T}$ and $I S O_{0.4}^{c a l}$, we take the following process to the conversion candidates in the JET samples (JET20 and JET50):

- Make $E_{T}$ distributions of the conversion electrons for each bin of $p_{T}$ and $I S O_{0.4}^{c a l}$,

- Convolute the above $E_{T}$ distributions and the $E_{T}$ dependence of $R_{\text {res }}$.

Figure 4.26 shows $R_{\text {res }}$ as a function of the track $p_{T}$ and $I S O_{0.4}^{c a l}$ for the JET samples. We see that the dependence on $p_{T}$ exists in $R_{\text {res }}$ but that on $I S O_{0.4}^{c a l}$ does not, which is the same tendency as $R_{\text {conv }}$. Then we calculate the corrected electron fake rate $R_{\text {fake }}^{\text {corr }}$ in accordance with Equation 4.11 by taking the above dependences into account for each JET sample. The obtained results are shown in Figure 4.27, where the original electron fake rates are superimposed for comparison. We use this corrected electron fake rate to evaluate the expected events for the fake electron background. 

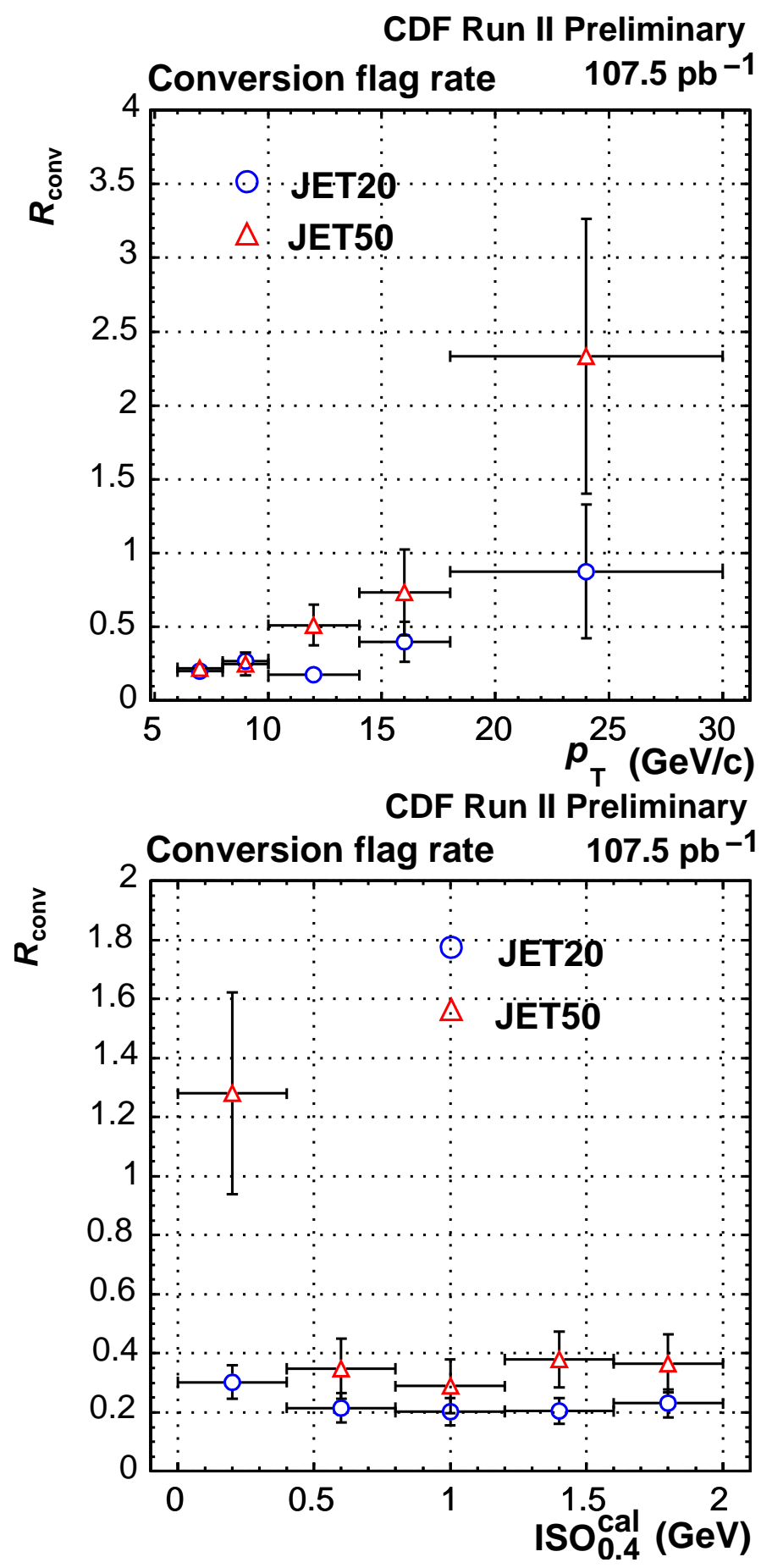

Figure 4.25: The conversion electron $p_{T}$ and $I S O_{0.4}^{c a l}$ dependence of the conversion flag rate for the JET20 and JET50 samples. 

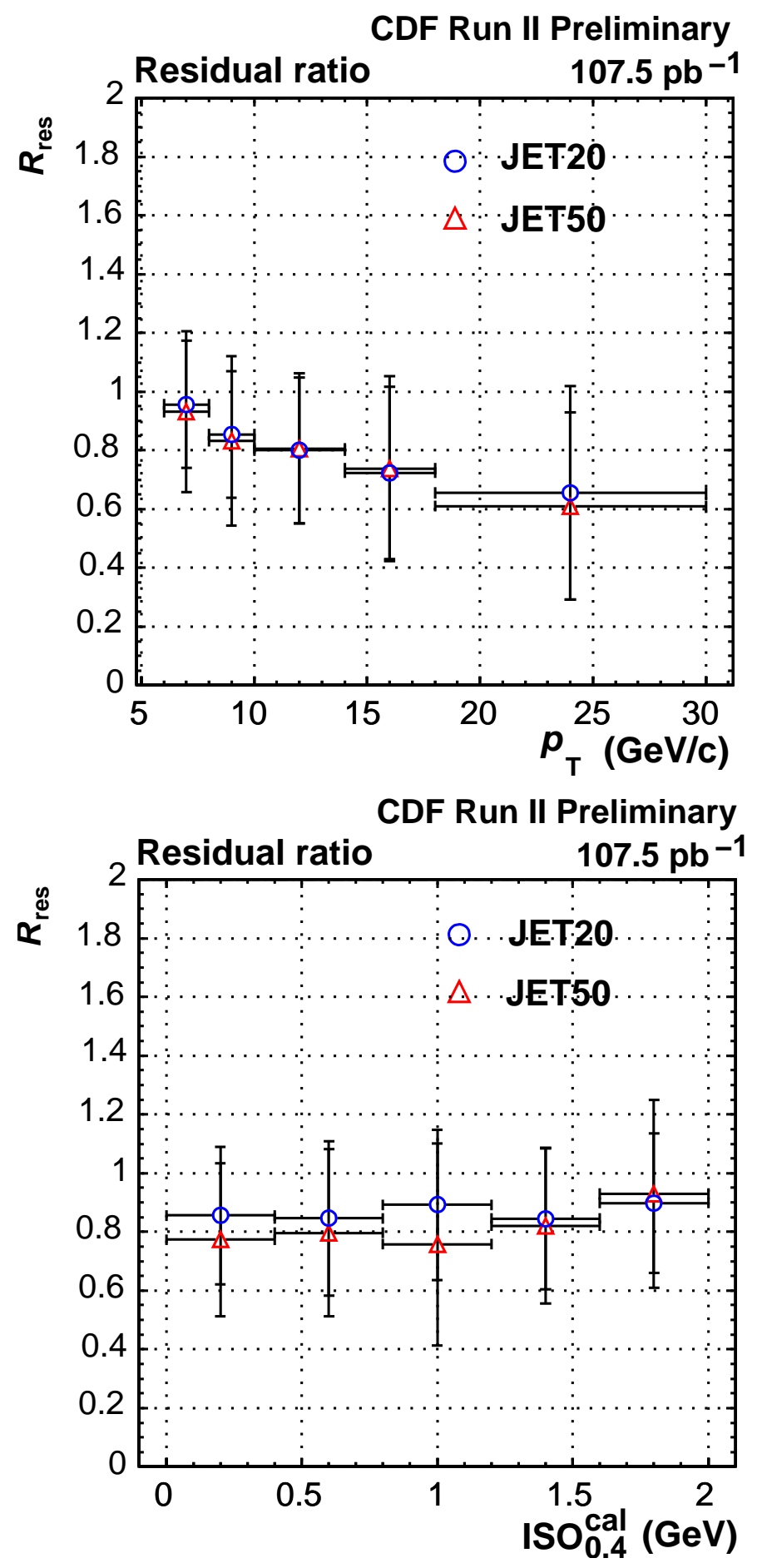

Figure 4.26: The conversion electron $p_{T}$ and $I S O_{0.4}^{c a l}$ dependence of the residual conversion ratio for the JET20 and JET50 samples. 

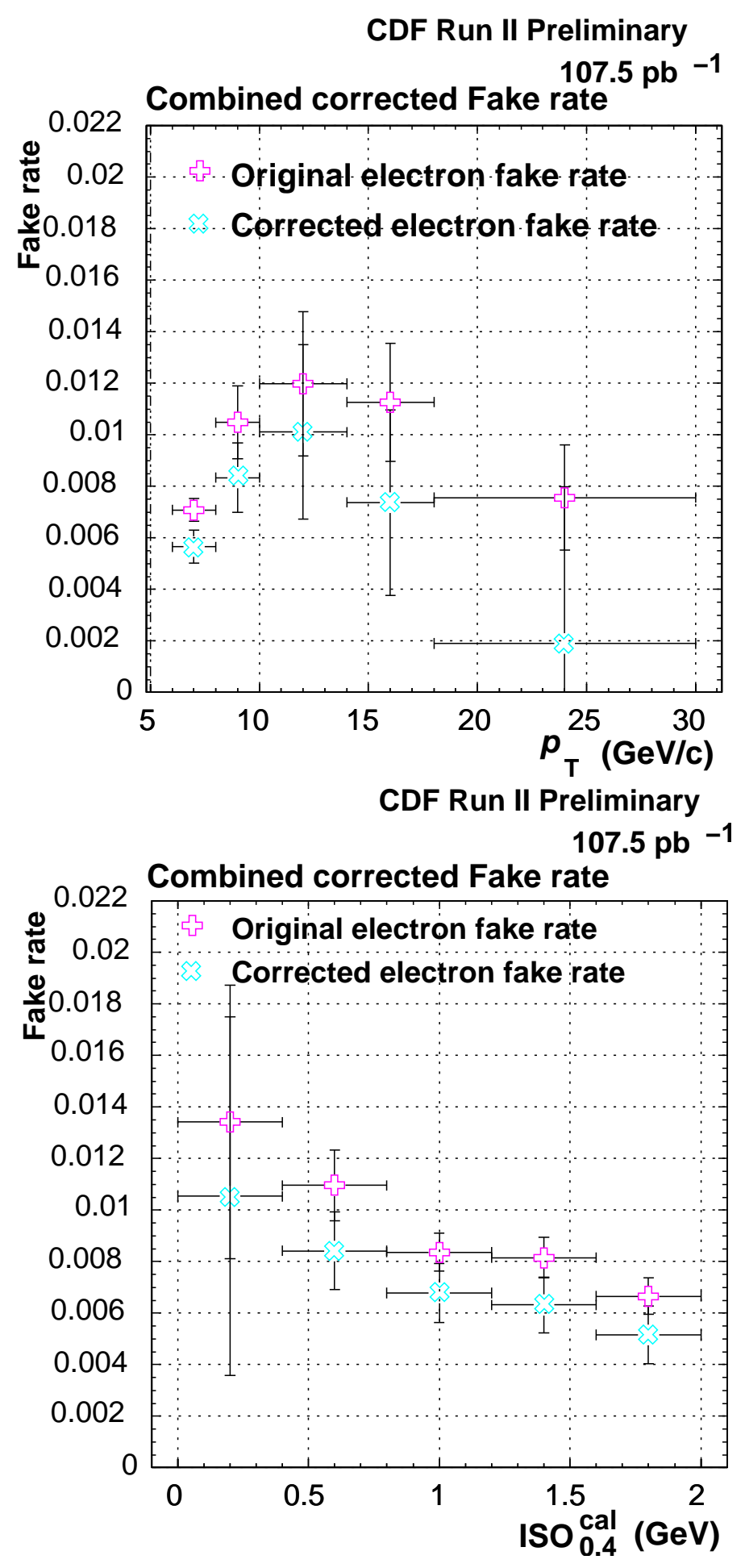

Figure 4.27: The $p_{T}$ and $I S O_{0.4}^{c a l}$ dependence of the original and corrected electron fake rates. The fake rates are combined values between the JET20 and JET50 samples. 


\subsection{Expected Event to Data Comparison}

In this section we compare the expected like-sign dilepton events with the observed ones for three lepton triggers listed below:

- Inclusive high- $p_{T}$ lepton (18 GeV lepton, electron and muon remake datasets),

- Lepton+Track (8 GeV lepton + $5 \mathrm{GeV}$ isolated track),

- Inclusive low- $p_{T}$ lepton (8 GeV lepton, calibration data-set),

For the lepton+track dataset we calculate the luminosity from the good run list [71]. The luminosity of the lepton+track dataset is $131.4 \mathrm{pb}^{-1}$. Since the low- $p_{T}$ lepton inclusive trigger is pre-scaled, we compare the number of $Z \rightarrow e e, \mu \mu$ events within the mass window from $81 \mathrm{GeV} / c^{2}$ to $101 \mathrm{GeV} / c^{2}$ between the inclusive high- $p_{T}$ lepton dataset and the inclusive low- $p_{T}$ lepton dataset to obtain the effective luminosity. The effective luminosity of the inclusive low- $p_{T}$ lepton dataset is estimated to be $42.6 \mathrm{pb}^{-1}$.

\subsubsection{2nd Leg Lepton}

We look into the second leg lepton in terms of its transverse momentum $p_{T}$, event $\eta$, and calorimeter isolation. The comparison between the expected and observed like-sign dilepton events is shown in Figure 4.28. We sum the like-sign $e e, e \mu$, and $\mu \mu$ events to increase the statistics in the plots. The major components of the like-sign dilepton events are the fake leptons and the residual conversions. The contribution of the background estimated by the Monte Carlo simulation of which the physics processes were described in Section 4.1 is found to be small. Figure 4.29 and 4.30 show the same distributions for the lepton+track and inclusive low- $p_{T}$ lepton dataset. For these dataset the $p_{T}$ cut position is lowered to $10 \mathrm{GeV} / c$ for the trigger leg and $6 \mathrm{GeV} / c$ for the second leg respectively in order to increase the statistics. Although the number of observed events is small, we see reasonable agreements within the statistical errors.

\subsubsection{Event Topological Variables}

We also look into the event topological variables such as missing transverse energy, lepton-pair opening angle in $x-y$ plane, and vector sum of lepton $p_{T}$ for the like-sign dilepton events. The results for the aforementioned three triggers are shown in Figures 4.31, 4.32, and 4.33. We see reasonable agreements again in the event topological variables within the statistical errors in spite of the small number of observed events. 

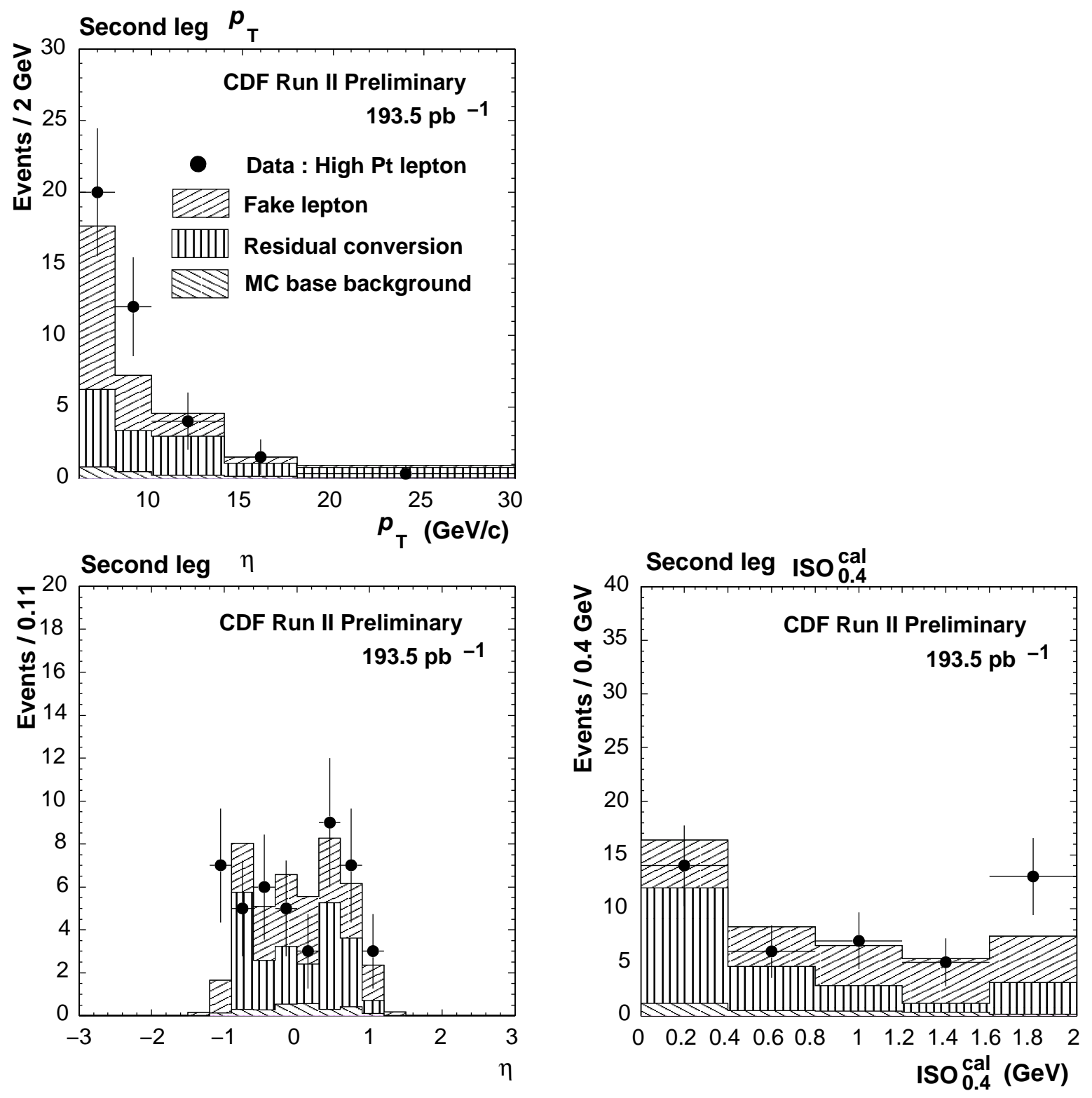

Figure 4.28: Expected and observed second leg $p_{T}$ (top left), event $\eta$ (bottom left), and $I S O_{0.4}^{\text {cal }}$ (bottom right) spectra for the inclusive high- $p_{T}$ trigger sample. 

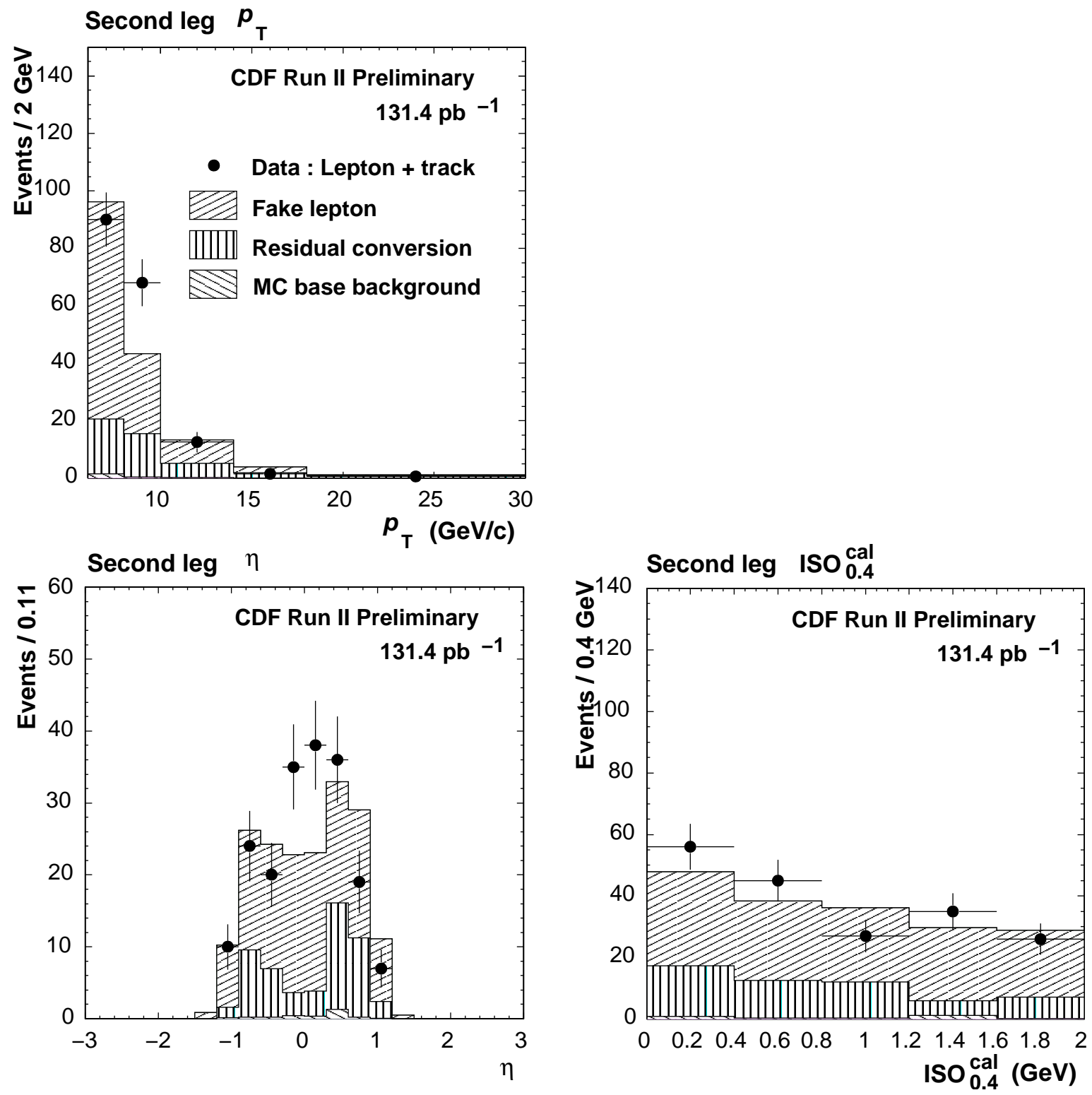

Figure 4.29: Expected and observed second leg $p_{T}$ (top left), event $\eta$ (bottom left), and $I S O_{0.4}^{c a l}$ (bottom right) spectra for the lepton+track trigger sample. 

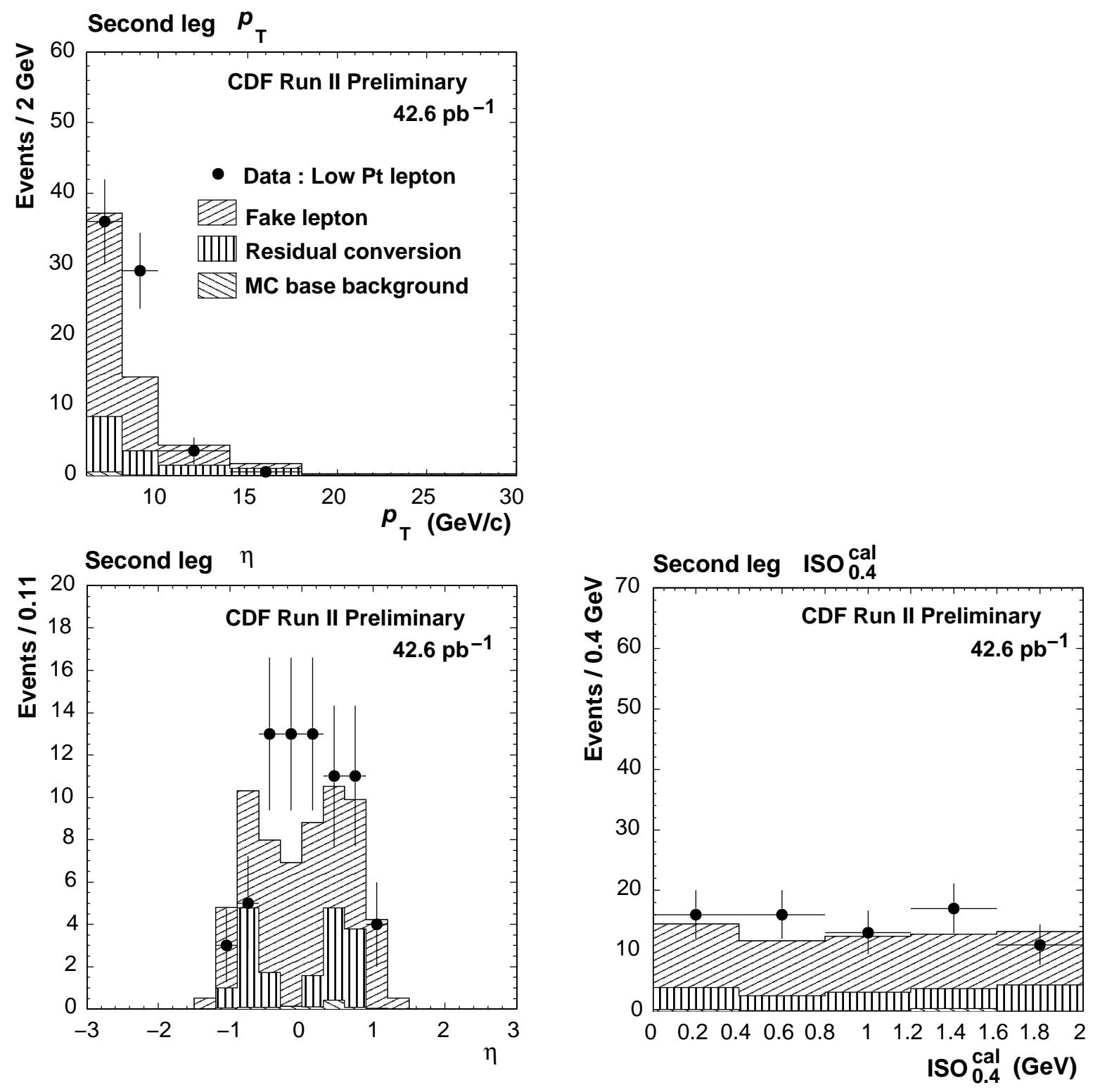

Figure 4.30: Expected and observed second leg $p_{T}$ (top left), event $\eta$ (bottom left), and $I S O_{0.4}^{c a l}$ (bottom right) spectra for the inclusive low- $p_{T}$ trigger sample. 

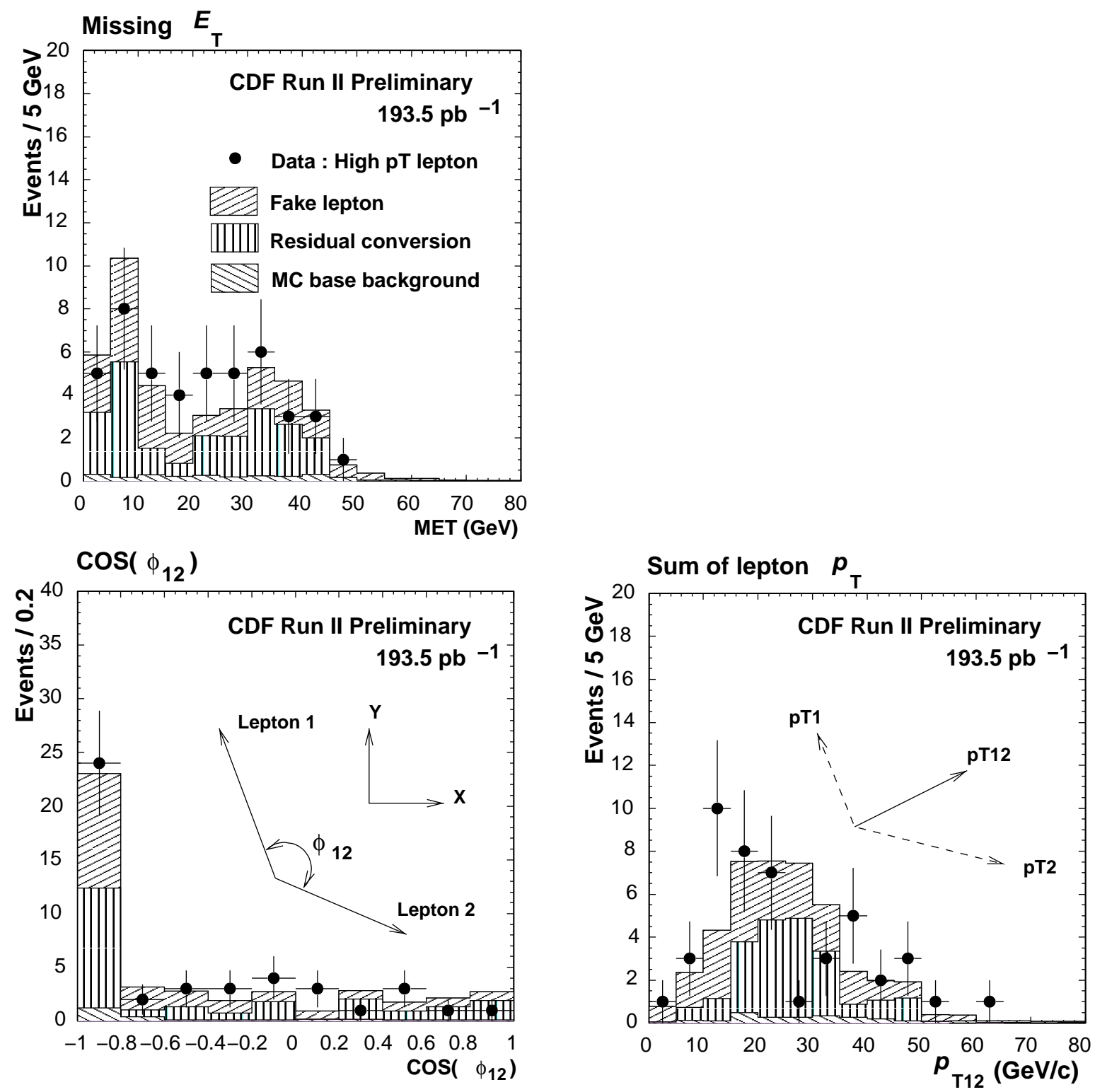

Figure 4.31: Expected and observed missing transverse $E_{T}$ (top left), lepton-pair opening angle (bottom left), and vector sum of lepton $P_{\mathrm{T} 12}$ (bottom right) for the like-sign dilepton events in the inclusive high- $p_{T}$ trigger sample. 

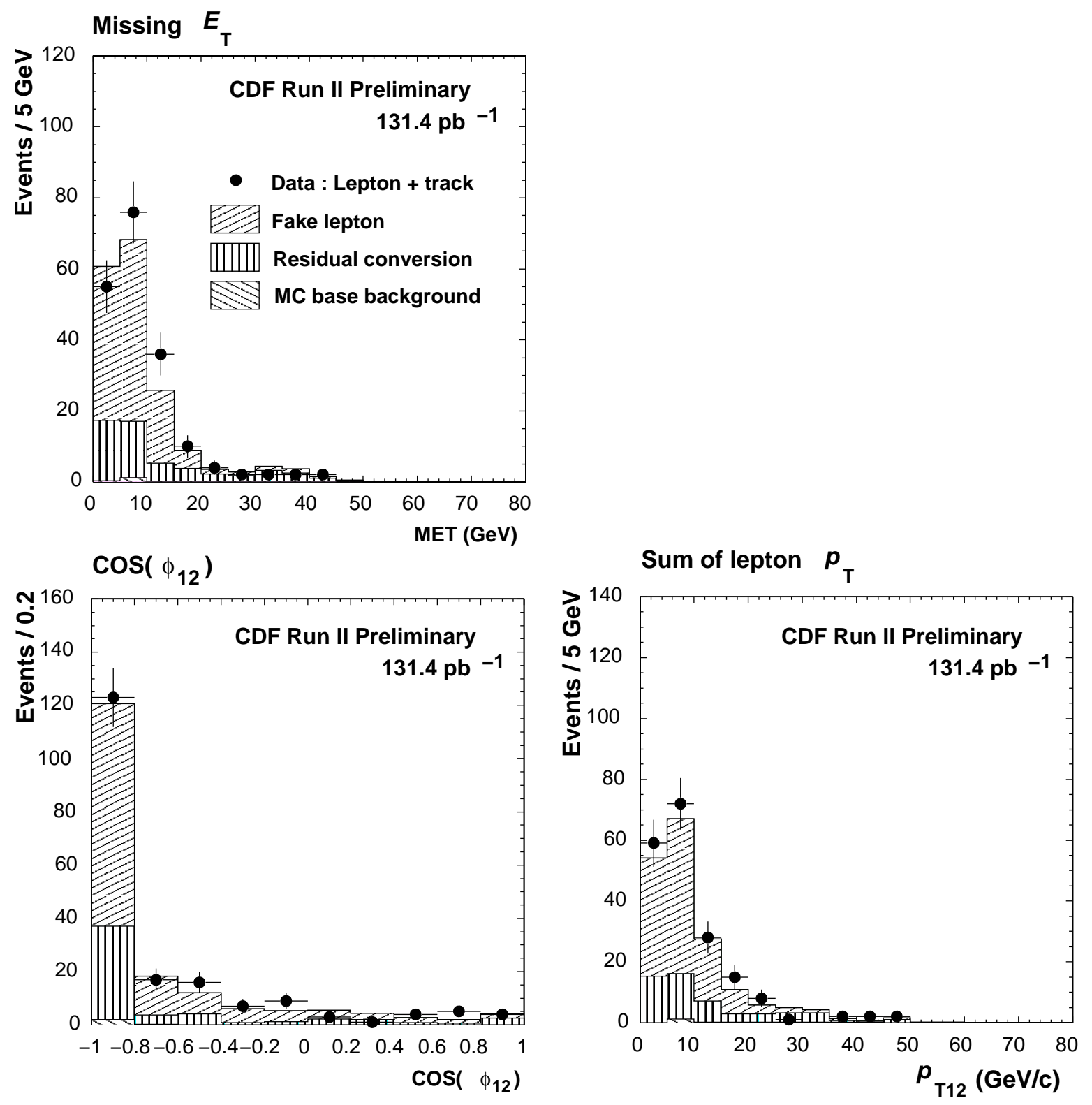

Figure 4.32: Expected and observed missing transverse $E_{T}$ (top left), lepton-pair opening angle (bottom left), and vector sum of lepton $P_{\mathrm{T} 12}$ (bottom right) for the like-sign dilepton events in the lepton+track data. 

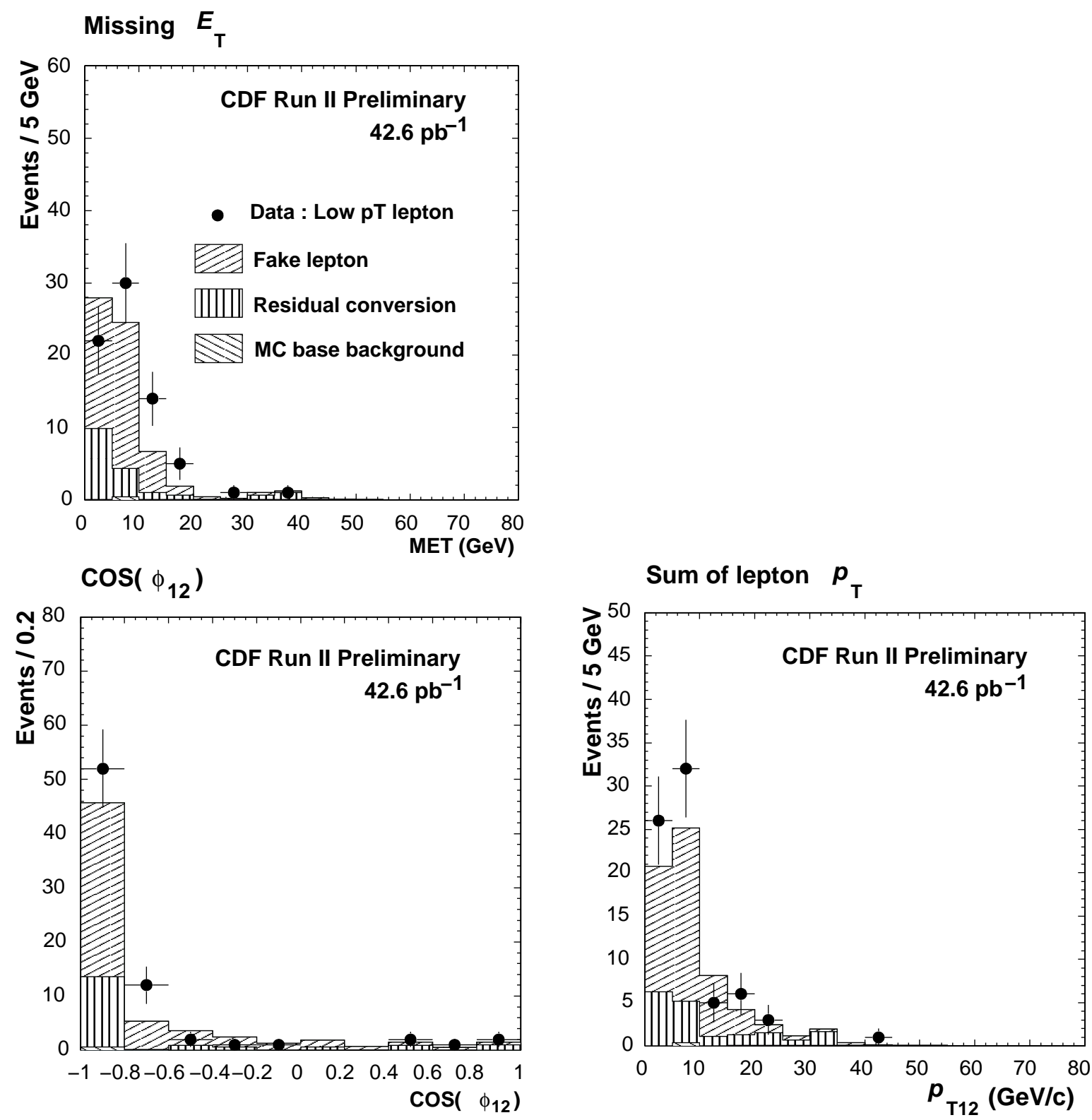

Figure 4.33: Expected and observed missing transverse $E_{T}, \cos \phi_{12}$ and $P_{\mathrm{T} 12}$ for inclusive low- $p_{T}$ data. 


\subsection{Summary of Background Estimation}

We found the major background components of the high- $p_{T}$ isolated like-sign dilepton are the fake leptons and the residual photon conversions. We measured the lepton fake rate per isolated-track using the JET samples. We separated the residual photon conversions from the fake leptons since we found the sample dependence of the conversion flag rate. We estimated the conversion tagging efficiency to obtain the contribution of the residual photon conversion, and the residual photon conversion was found to account for a large contribution to the total background as well as the fake lepton. We compared the number of expected and observed events for the lepton samples collected with the three different triggers and we saw reasonable agreements. This means that the contribution of other fake lepton components, leptons from $b$-jets, for example, are the same between the JET samples and the like-sign dilepton events. 


\section{Chapter 5}

\section{Topological Cut}

We describe a search for the neutral Higgs production associated with the $W$ using high- $p_{T}$ isolated like-sign dilepton events in the $193.5 \mathrm{pb}^{-1}$ CDF Run II data. We determine the signal box in the plane of the second leading lepton $p_{T}$ versus the vector sum of $p_{T}$ 's of the two leptons as an event topological cut.

\subsection{Determination of Signal Region}

We decide a signal box in the plane of the second leg $p_{T}\left(p_{T 2}\right)$ and the vector sum of lepton's $p_{T}\left(p_{T 12}\right)$ as a topological cut to enhance the signal significance. We referred to the Run I analysis of the inclusive high- $p_{T}$ like sign dilepton search [68]. The $p_{T 2}$ vs $p_{T 12}$ scatter plots of the signal $W H$ Monte Carlo (MC) and the backgrounds are shown in Figure 5.1. The signal and the diboson backgrounds are distributed up to the high $p_{T 2}$ and $p_{T 12}$ region. The fake leptons are localized at the low $p_{T 2}$ and $p_{T 12}$ region. The residual photon conversions are distributed in the low $p_{T 12}$ region. We use the $110 \mathrm{GeV} / c^{2}$ bosophilic Higgs and the $160 \mathrm{GeV} / c^{2}$ SM Higgs MC events as signal samples and the total expected events as background samples, then look for the region to improve the signal significance $(S / \sqrt{B})$ as much as possible to the extent that we can expect at least about one-signal event after applying the cuts to the data with the base-projection integrated luminosity of $4.4 \mathrm{fb}^{-1}$.

Figure 5.2 shows the change of $S / \sqrt{B}$ as a function of the $p_{T 2}$ and $p_{T 12}$ cut values. The cut value pair which gives the maximum $S / \sqrt{B}$ is $\left(p_{T 2}, p_{T 12}\right)=(16,35) \mathrm{GeV} / c$ for the case of Higgs mass being less than $160 \mathrm{GeV} / c^{2}$ and $(18,35) \mathrm{GeV} / c$ for the case of Higgs mass being equal or more than $160 \mathrm{GeV} / c^{2}$. We also check the change of the number of expected signal events in accordance with the cut values as shown in Figure 5.3. The number of expected events is 1.5 for the $110 \mathrm{GeV} / c^{2}$ case and 0.74 for the 160 $\mathrm{GeV} / c^{2}$ case. We find the number of expected signal events is not too small in both cases. 
We note that this discussion is not really about an optimization since the result is subject to the statistical fluctuation of the sample used to derive the background expectations. We should therefore read only the trend of the significance change with different cut values. Our choice of the signal region is rather connected to the fact that we would barely observe one event on the average in the full data of $4.4 \mathrm{fb}^{-1}$.

\subsection{Checks around the Signal Box}

We divide the $p_{T 2}$ vs $p_{T 12}$ plane into four regions: $\mathrm{A}, \mathrm{B}, \mathrm{C}$, and signal box, which are indicated in Figure 5.4. We compare the number of expected events with that of observed ones in these regions except the signal box as a sanity test of background estimation. Table 5.2 shows the breakdown of background components and the number of observed events for each region. The statistical errors of the total background estimates reflect the MC statistics and the sample statistics to which the fake rate and the residual-conversion ratio are applied, while the systematic errors originate from the error of the fake rate and the residual-conversion ratio. The major component in the region $\mathrm{A}$ and the signal box are the residual photon conversion, while the fake lepton and the residual photon conversion dominate in the other regions. Figure 5.4 shows the observed $p_{T 2}$ vs $p_{T 12}$ scatter plot. In each region we see reasonable agreements.

\begin{tabular}{l}
\hline Second leg $p_{T}>16 \mathrm{GeV} / c\left(\right.$ Higgs Mass $\left.<160 \mathrm{GeV} / c^{2}\right)$ \\
Second leg $p_{T}>18 \mathrm{GeV} / c\left(\right.$ Higgs Mass $\left.\geq 160 \mathrm{GeV} / c^{2}\right)$ \\
Vector sum of lepton $p_{T}>35 \mathrm{GeV} / c$ \\
\hline \hline
\end{tabular}

Table 5.1: Topological cuts 
CDF Run II Preliminary

\begin{tabular}{c|cccccccc}
\hline \hline Region & $W Z$ & $Z Z$ & $t \bar{t}$ & $W Q \bar{Q}$ & $W W$ & $Z / \gamma^{*}$ & Fake & Residual Conv \\
\hline A & 0.060 & 0.0078 & 0.0048 & 0.0036 & 0.0011 & 0.29 & 0.91 & 4.9 \\
B & 0.028 & 0.0050 & 0.019 & 0.31 & 0.0012 & 0.93 & 15.8 & 12.2 \\
C & 0.054 & 0.0060 & 0.036 & 0.29 & 0.0040 & 0.33 & 4.0 & 3.4 \\
A + B + C & 0.28 & 0.032 & 0.069 & 0.61 & 0.0096 & 1.6 & 20.8 & 21.0 \\
Signal box & 0.14 & 0.013 & 0.0097 & 0.0033 & 0.0034 & 0.051 & 0.12 & 0.61 \\
\hline \hline
\end{tabular}

\begin{tabular}{c|cccc}
\hline \hline Region & WH (110) & WH (160) & Total & Observed event \\
\hline A & 0.039 & 0.011 & $6.2 \pm 2.0 \pm 1.4$ & 3 \\
B & 0.023 & 0.0013 & $29.3 \pm 3.8 \pm 3.3$ & 30 \\
C & 0.034 & 0.0065 & $8.1 \pm 2.1 \pm 1.0$ & 12 \\
A + B + C & 0.096 & 0.013 & $43.6 \pm 3.3 \pm 5.1$ & 45 \\
Signal box & 0.056 & 0.030 & $0.95 \pm 0.61 \pm 0.18$ & \\
\hline \hline
\end{tabular}

Table 5.2: Breakdown of background components and observed events for each region. 

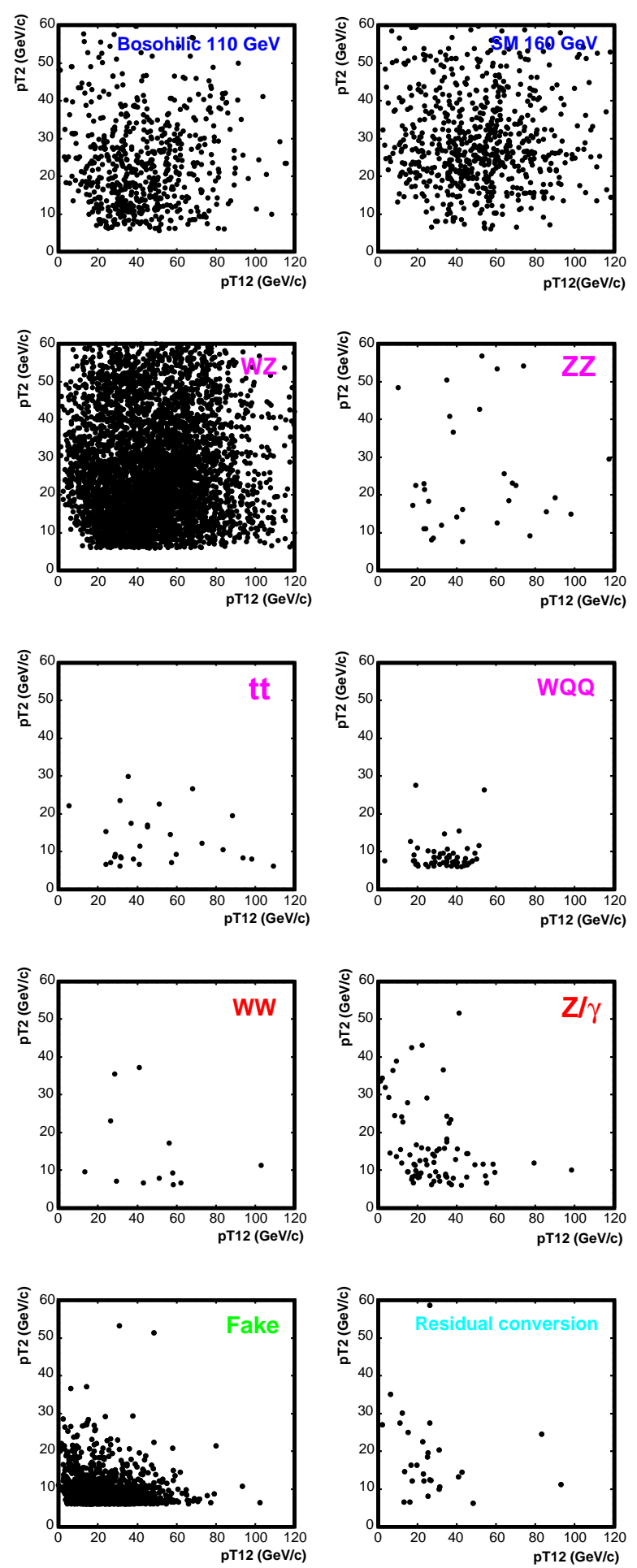

Figure 5.1: $p_{T 2}$ vs $p_{T 12}$ scatter plots of the signal and backgrounds. 

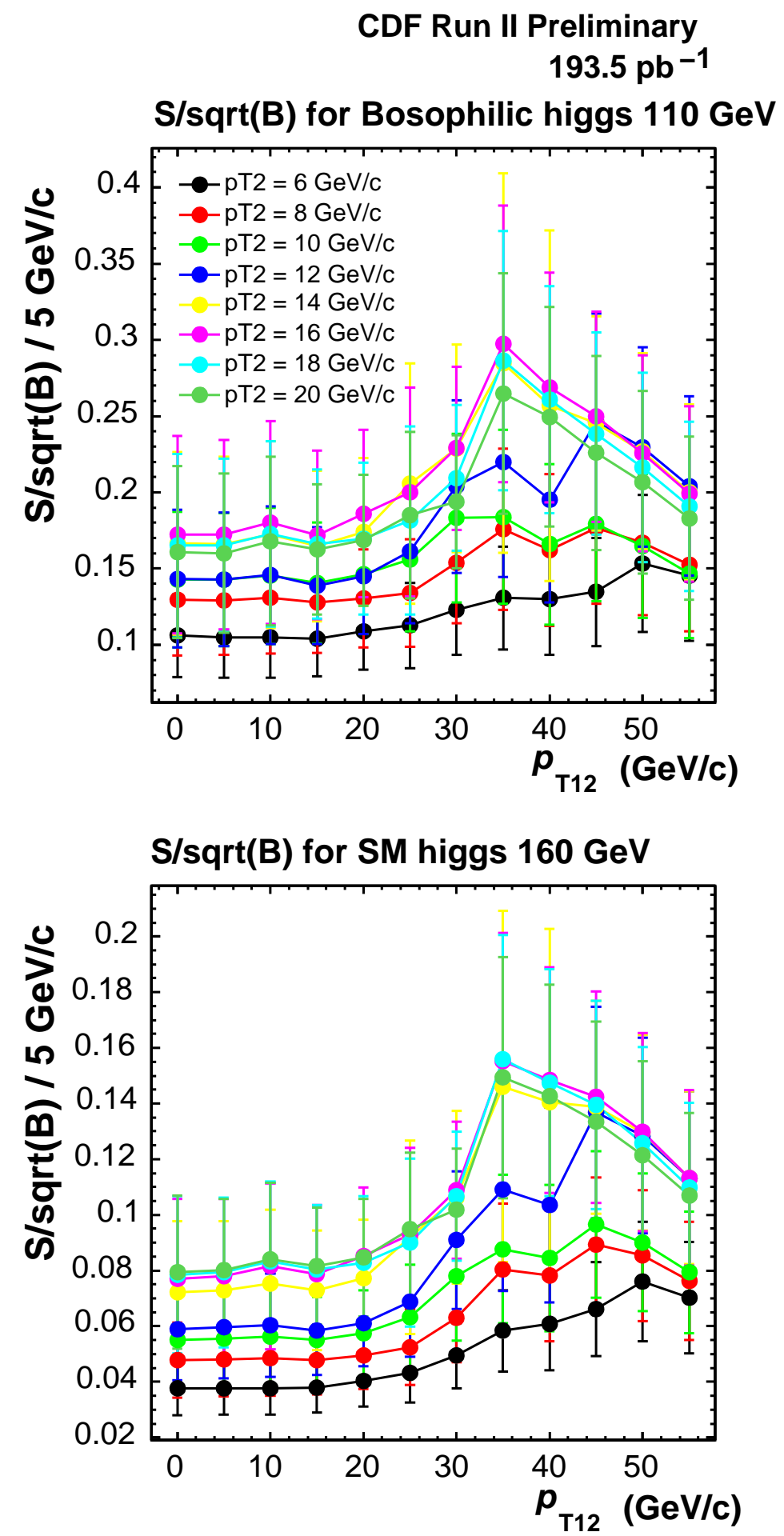

Figure 5.2: Upper (Lower) plot shows the $S / \sqrt{B}$ as a function of $p_{T 12}$ cut value for the $110 \mathrm{GeV} / c^{2}$ bosophilic Higgs (160 GeV/c $c^{2}$ SM Higgs). 

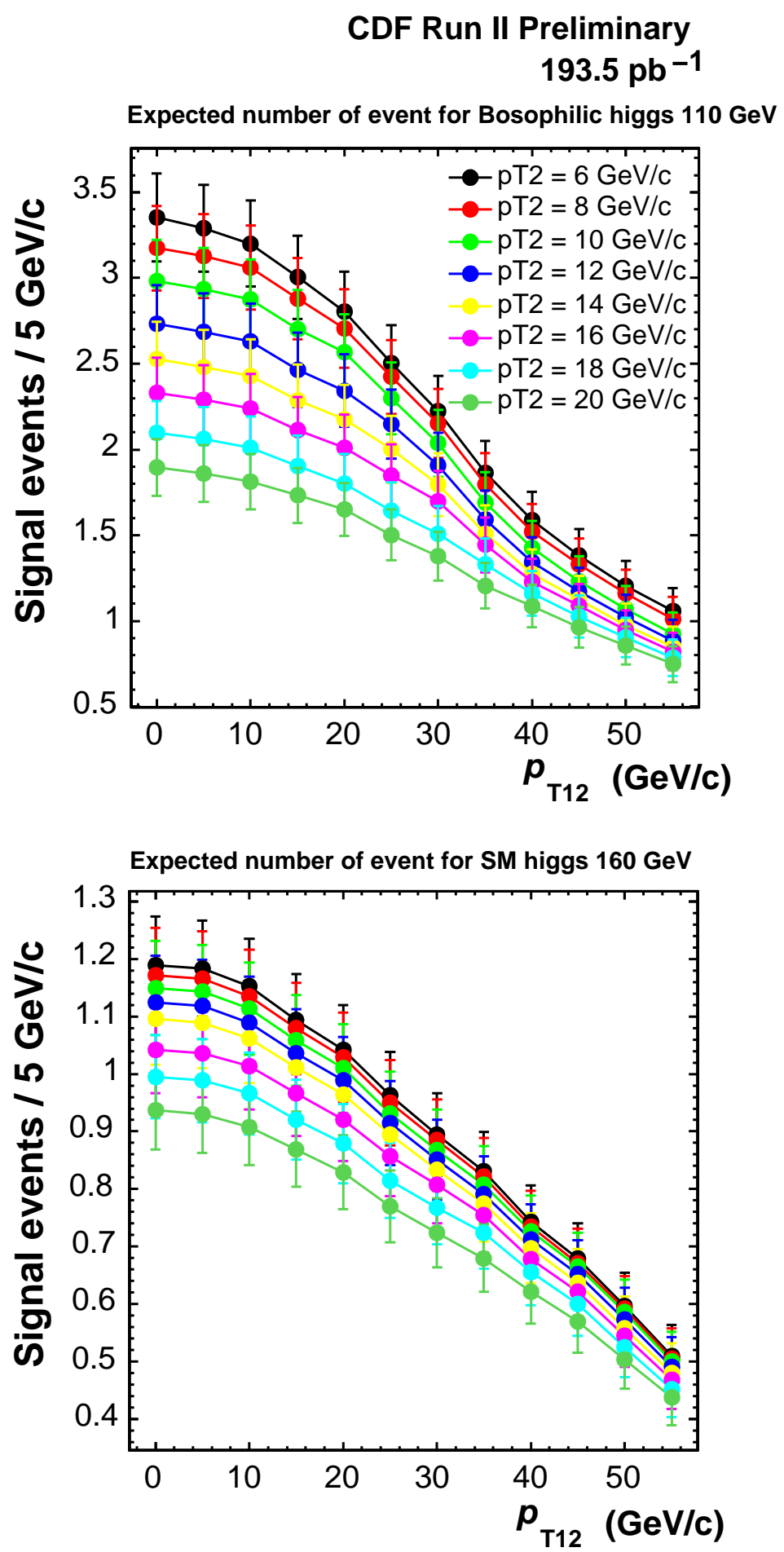

Figure 5.3: Upper (Lower) plot shows the number of expected signal events as a function of $p_{T 12}$ cut value for the $110 \mathrm{GeV} / c^{2}$ bosophilic Higgs ( $160 \mathrm{GeV} / c^{2} \mathrm{SM}$ Higgs). 


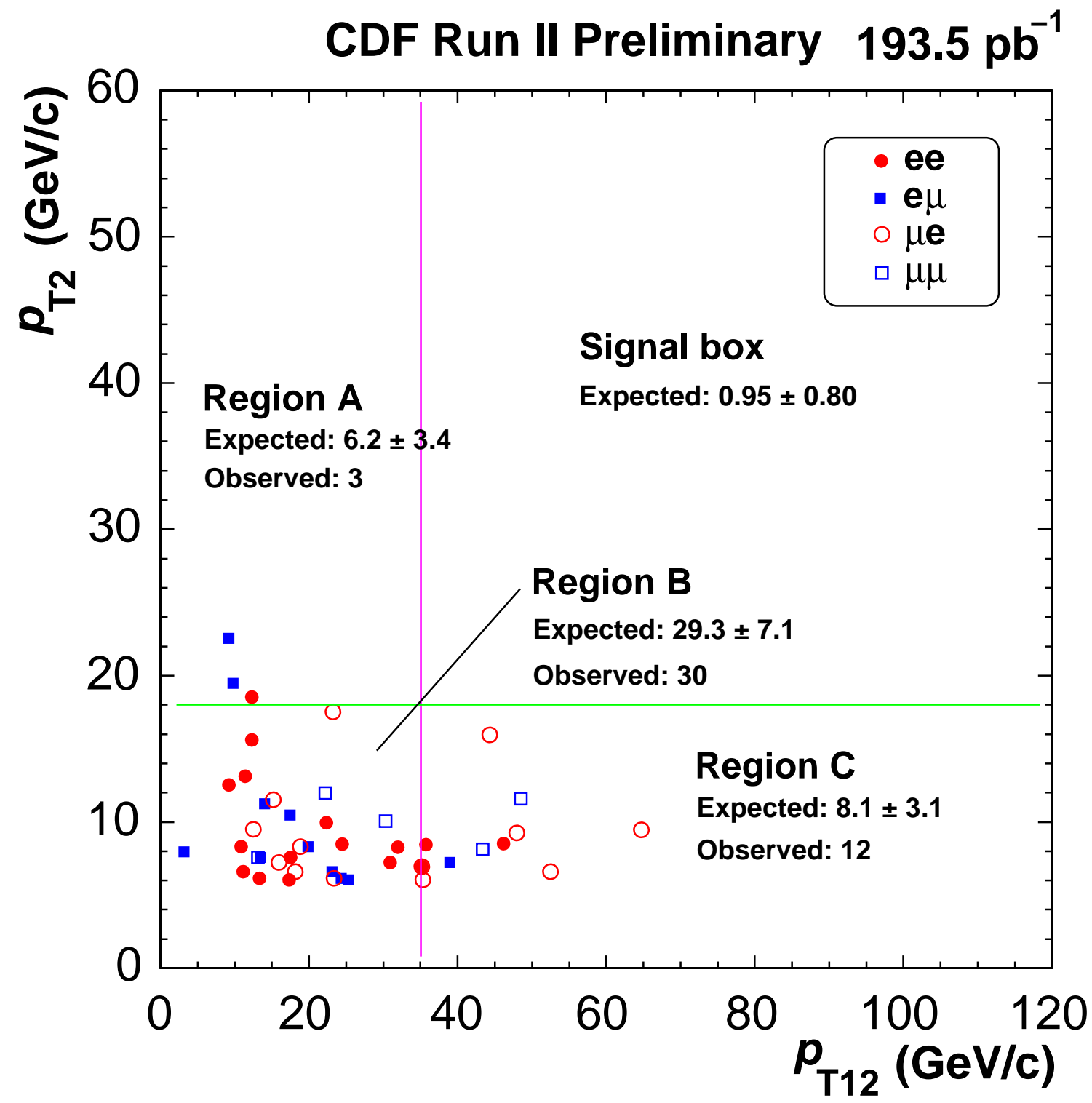

Figure 5.4: $p_{T 2}$ vs $p_{T 12}$ scatter plot. The region $\mathrm{A}$ is defined by $p_{T 2}>18 \mathrm{GeV} / c$ and $p_{T 12} \leq 35 \mathrm{GeV} / c$. The region $\mathrm{B}$ is defined by $p_{T 2} \leq 18 \mathrm{GeV} / c$ and $p_{T 12} \leq 35 \mathrm{GeV} / c$. The region $\mathrm{C}$ is defined by $p_{T 2} \leq 18 \mathrm{GeV} / c$ and $p_{T 12}>35 \mathrm{GeV} / c$. The signal box is defined by $p_{T 2}>18 \mathrm{GeV} / c$ and $p_{T 12}>35 \mathrm{GeV} / c$. Closed (Open) points correspond to the inclusive high $p_{T}$ electron (muon) sample. The differentiation between $e \mu$ and $\mu e$ is meant to indicate the first one is the leading lepton. 


\section{Chapter 6}

\section{Signal Acceptance}

The total detection efficiency can be written as

$$
\begin{aligned}
& \varepsilon_{\mathrm{tot}}=\sum_{i} A^{(i)} \cdot \varepsilon_{\mathrm{iso}}^{(i)} \cdot \varepsilon_{\mathrm{ID}}^{(i)} \cdot \varepsilon_{\mathrm{dil}}^{(i)} \cdot \varepsilon_{\mathrm{topo}}^{(i)} \cdot \varepsilon_{\mathrm{trig}}^{(i)}=A \cdot \varepsilon_{\mathrm{iso}} \cdot \varepsilon_{\mathrm{ID}} \cdot \varepsilon_{\mathrm{dil}} \cdot \varepsilon_{\mathrm{topo}} \cdot \varepsilon_{\mathrm{trig}} \text {, } \\
& \left(\begin{array}{ll}
A^{(i)} & : \text { geometrical and kinematical acceptance } \\
\varepsilon_{\text {iso }}^{(i)} & : \text { isolation cut efficiency } \\
\varepsilon_{\text {ID }}^{(i)} & : \text { lepton identification efficiency } \\
\varepsilon_{\text {dil }}^{(i)} & : \text { dilepton selection efficiency } \\
\varepsilon_{\text {topo }}^{(i)}: \text { topological cut efficiency } \\
\varepsilon_{\text {trig }}^{(i)}: \text { trigger efficiency }
\end{array}\right)
\end{aligned}
$$

where $i$ runs over different types of lepton objects such as CEM-CEM, CEM-CMUP, CEM-CMX, and so on. The complete cuts are listed in Tables 3.3, 3.4, and 5.1. The order of multiplication has a meaning and we calculate the efficiencies sequentially. The relation between, for example, $\varepsilon_{\mathrm{ID}}^{(i)}$ and $\varepsilon_{\mathrm{ID}}$ in Equation (6.1), can be understood by:

$$
\varepsilon_{\mathrm{ID}}=\frac{\sum_{i} A^{(i)} \cdot \varepsilon_{\mathrm{iso}}^{(i)} \cdot \varepsilon_{\mathrm{ID}}^{(i)}}{\sum_{i} A^{(i)} \cdot \varepsilon_{\mathrm{iso}}^{(i)}} .
$$

The $A$ and $\varepsilon_{\text {topo }}$ is estimated mainly using MC. We introduce scale factors for the event-vertex cut efficiency included in $A, \varepsilon_{\text {iso }}$, and $\varepsilon_{\mathrm{ID}}$. The trigger efficiency $\varepsilon_{\text {trig }}$ is derived from data, and $\varepsilon_{\text {dil }}$ is a mixture of data- and MC-driven calculations.

\subsection{Signal Monte Carlo Samples}

We make the signal MC samples by PYTHIA of version 6.2 [41]. The process of our interest is $W H \rightarrow W W^{*} W^{*} \rightarrow$ dilepton or trilepton, where the lepton is electron 
or muon only. The branching ratio of $W W^{*} W^{*}$ into dilepton or trilepton is $12 \%$ (See Figures 6.1 and 6.2). We force $W H$ events to decay into $W W^{*} W^{*}$ and select $\geq 2$ lepton events at generator level. We change the Higgs boson mass from 90 to 200 $\mathrm{GeV} / c^{2}$ and make $40 \mathrm{~K}$ events for each Higgs boson mass point. On the other hand we also make $W H+Z H \rightarrow V V^{*} V^{*} \rightarrow$ dilepton or trilepton to investigate the like-sign dilepton through various decay modes, where $V$ is $W$ or $Z$ boson. In this sample we also count electrons and muons from tau leptons. The branching ratio of $V V^{*} V^{*} \rightarrow \geq$ 2 leptons is $15.5 \%$. We also change the Higgs boson mass from 90 to $200 \mathrm{GeV} / c^{2}$ and make $40 \mathrm{~K}$ events for each Higgs boson mass point.

\subsection{Geometrical and Kinematical Acceptance}

The efficiency of the event-vertex cut is obtained from data [78]. We apply the same cut to MC samples too, then need to correct for a small difference of the efficiency between MC and data. We compare the data-derived efficiency with those from various MC samples as listed in Table 6.1. The scale factors (data/MC) are almost common between the MC samples as expected. We use $0.978 \pm 0.006$ as the scale factor for the efficiency of the $\left|z_{\mathrm{vtx}}\right|<60 \mathrm{~cm}$ cut. The other geometrical and kinematical cut efficiencies are calculated from the signal MC samples. Figure 6.3 shows the $p_{T}$ of dileptons passing geometrical and kinematic cuts. We see that the second leg $p_{T}$ is a variable which is influenced by the Higgs boson mass strongly. The Higgs boson mass dependences of $A^{(i)}$ is shown in Figure 6.4 for each type of lepton pair. The total dilepton efficiencies change from $15 \%$ to $24 \%$ according to the Higgs boson mass. The efficiencies are also shown in Figure 6.4 for the $W H+Z H \rightarrow V V V \rightarrow$ dilepton case in which we see that the $e e$ and $\mu \mu$ channels are relatively enhanced compared to $e \mu$ since we have contributions from $Z \rightarrow \ell \ell$ of the $Z H$ production.

\begin{tabular}{c|ccccc}
\hline \hline Monte Carlo & $W H 110$ & $W H 140$ & $W H 160$ & $Z \rightarrow e e$ & $Z \rightarrow \mu \mu$ \\
\hline$\varepsilon\left(z_{\text {vtx }}\right)$ & $0.971 \pm 0.002$ & $0.971 \pm 0.001$ & $0.970 \pm 0.001$ & $0.974 \pm 0.001$ & $0.975 \pm 0.001$ \\
Scale Factor & $0.979 \pm 0.006$ & $0.979 \pm 0.006$ & $0.980 \pm 0.007$ & $0.974 \pm 0.006$ & $0.975 \pm 0.006$ \\
\hline Combined & \multicolumn{5}{|c}{$0.978 \pm 0.006$} \\
\hline \hline
\end{tabular}

Table 6.1: $z_{\mathrm{vtx}}$ cut efficiencies and scale factors for the different Monte Carlo samples. We refereed $0.951 \pm 0.006$ as data-derived $z_{\mathrm{vtx}}$ cut efficiency [78]. Combined value is the average of five different Monte Carlo sample. 

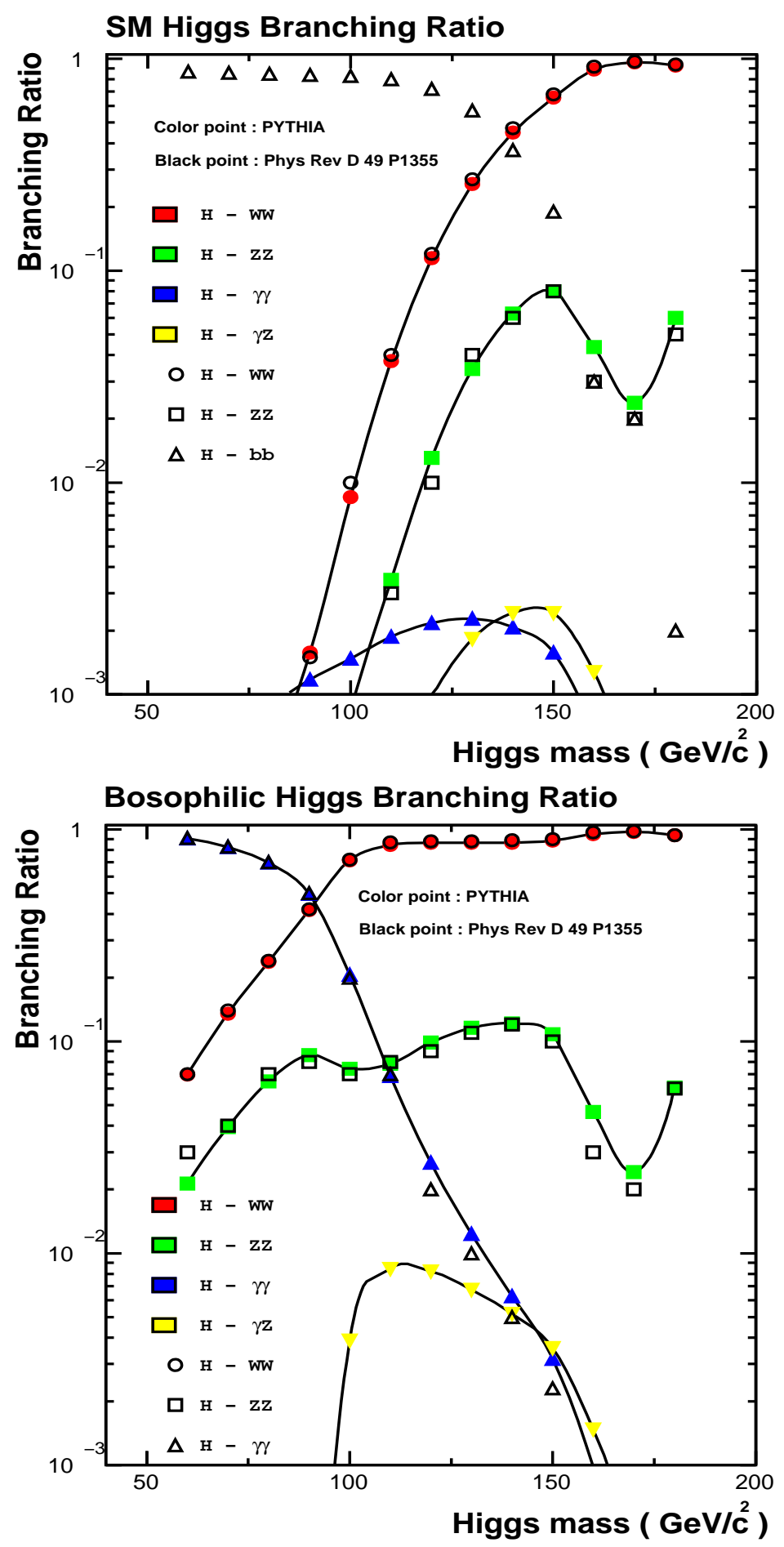

Figure 6.1: Upper (Lower) plot shows the standard model (bosophilic) Higgs boson branching ratio. 


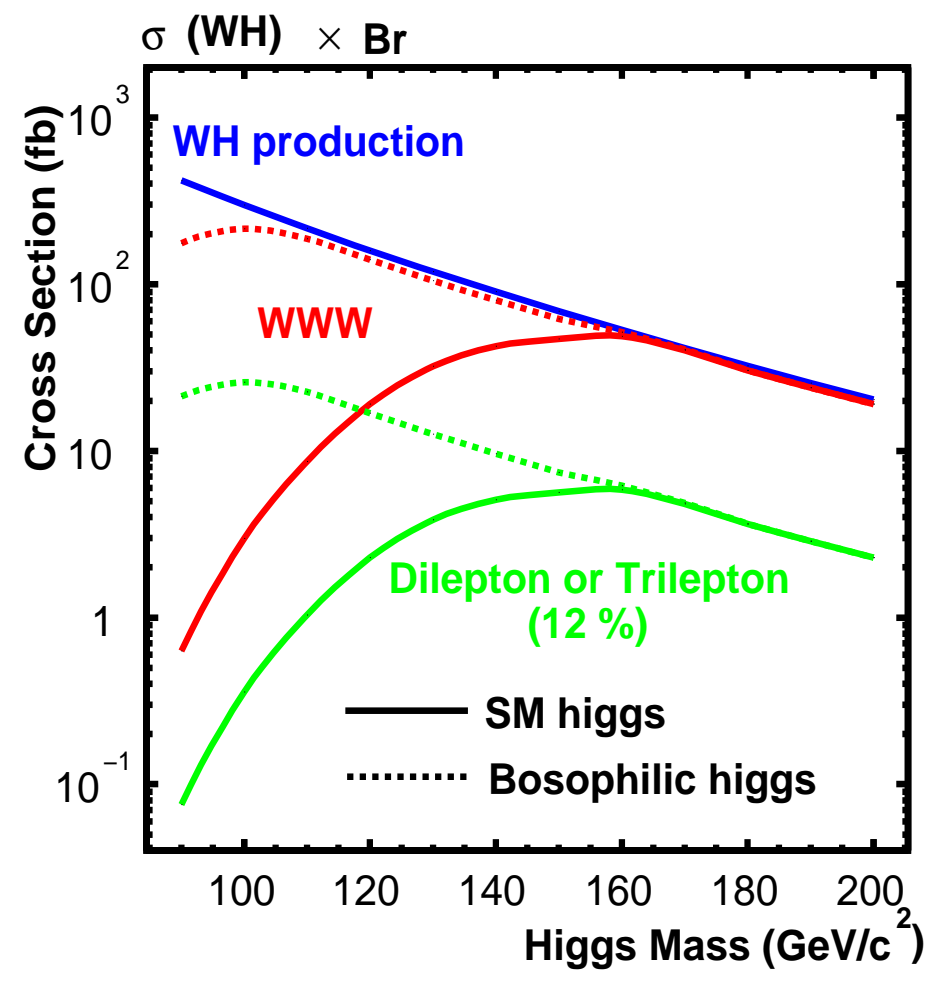

Figure 6.2: Signal cross section after multiplying branching fractions. The Solid line corresponds to the SM Higgs boson and the broken line corresponds to the bosophilic Higgs boson. 

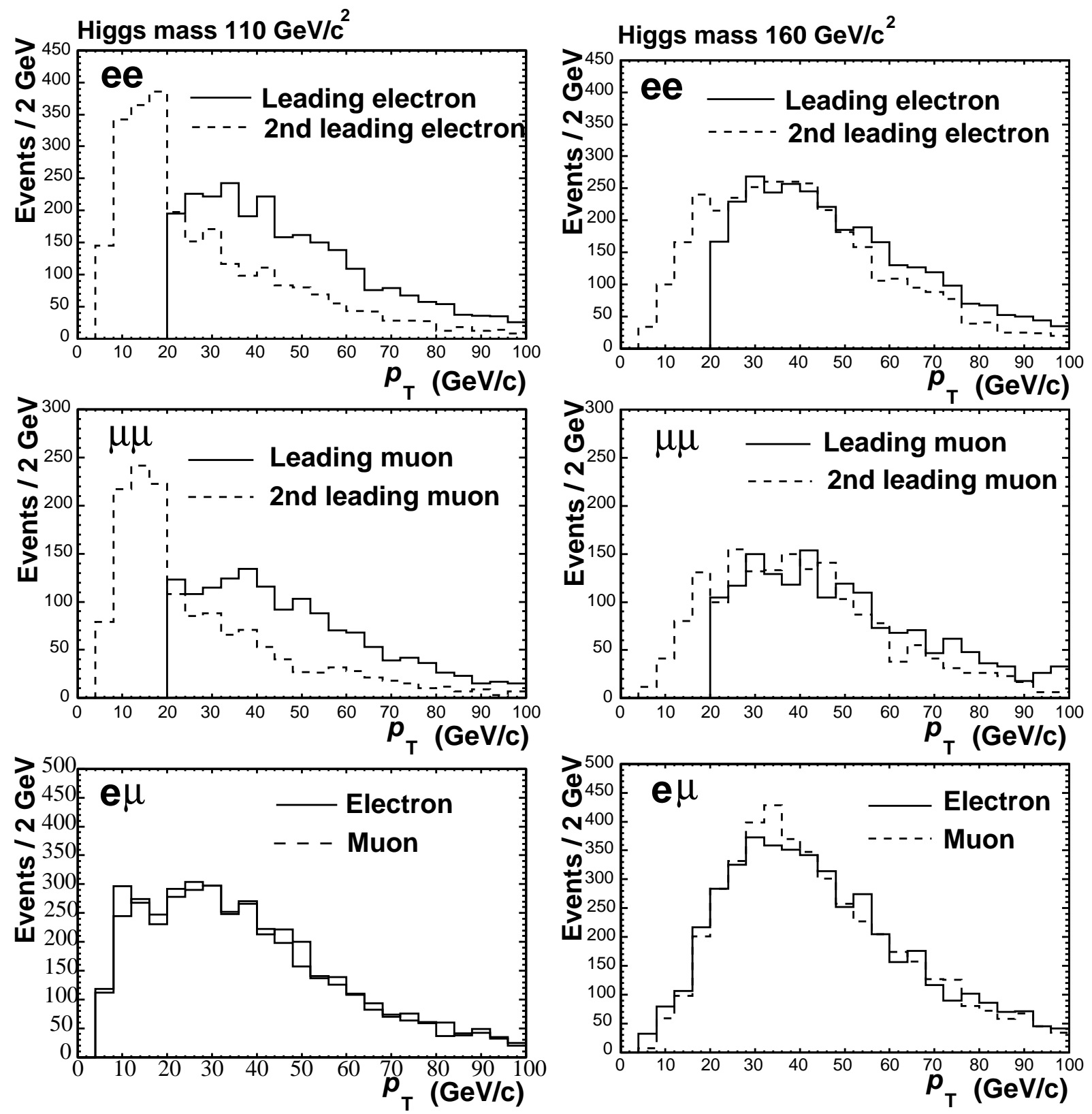

Figure 6.3: Leading lepton and second leading lepton $p_{T}$ distributions for each dilepton type (ee, $\mu \mu$, and $e \mu)$. Left (Right) plots correspond to the $110 \mathrm{GeV} / c^{2}\left(160 \mathrm{GeV} / c^{2}\right)$ Higgs boson mass Monte Carlo sample. 


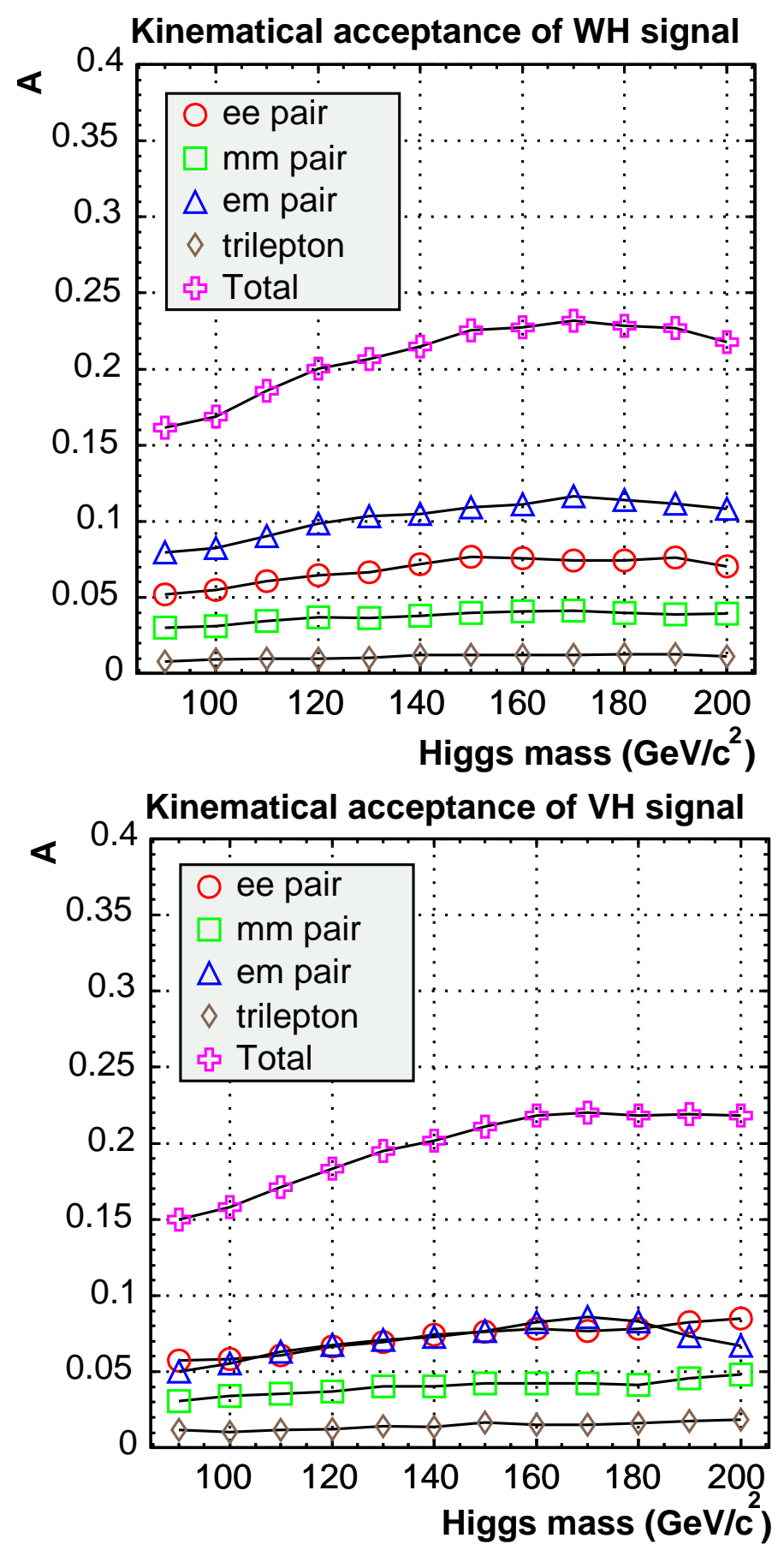

Figure 6.4: Upper (Lower) plot shows geometrical and kinematical cut efficiency for each dilepton type for the $W H \rightarrow W W^{*} W^{*} \rightarrow$ dilepton $\left(W H+Z H \rightarrow V V^{*} V^{*} \rightarrow\right.$ dilepton) signal Monte Carlo samples. Circles, squares, triangles, and diamonds correspond to the dielectron channel, dimuon channel, $e \mu$ channel, and trilepton channel respectively. 


\subsection{Isolation Cut Efficiency}

We use the traditional calorimeter isolation with the cone size of $0.4, I S O_{0.4}^{\text {cal }}$, for the lepton selection. The isolation $I S O_{0.4}^{\text {cal }}$ is defined as

$$
\begin{gathered}
E_{T}^{\text {clust }}=E_{T}^{\text {seed }}+E_{T}^{(\eta+1)}+E_{T}^{(\eta-1)}, \\
I S O_{0.4}^{\text {cal }}=\left(\sum_{\Delta R<0.4} E_{T}^{\text {cal }}\right)-E_{T}^{\text {clust }} .
\end{gathered}
$$

We use this isolation for the electron and muon objects. The isolation cut efficiency is calculated by scaling the MC-derived efficiency with a scale factor. The scale factor is estimated by the comparison between the $Z \rightarrow \ell \ell$ isolation efficiency of data and that of the MC samples. The second legs of $Z \rightarrow \ell \ell$ after applying the geometrical and kinematical cuts are used for the comparison. We calculate the efficiency by the following formula according to the combination of the lepton pair.

$$
\begin{gathered}
\text { Same type lepton pair }: \frac{2 \cdot N_{\text {pass }}}{N_{\text {pass }}+N_{\text {no-bias }}} \\
\text { Different type lepton pair }: \frac{N_{\text {pass }}}{N_{\text {no-bias }}}
\end{gathered}
$$

Table 6.2 shows the isolation cut efficiencies and the scale factors for each lepton type. The isolation distribution and the $p_{T}$ and $\eta$ dependences are shown in Figures 6.5 and 6.6. We see some difference in the isolation distribution between the Monte Carlo and data. However since we set the isolation cut to $2 \mathrm{GeV}$, the difference of the efficiency is small. The errors of the scale factors are calculated from the statistical errors of efficiencies obtained from the MC samples and data. The signal isolation distribution is shown in Figures 6.7 to 6.9 for each lepton pair. The isolation distribution is different from that of $Z \rightarrow \ell \ell$ and there is a mass dependence of the efficiency. This is due to the effect of jets from one of $W$ 's produced in the signal process. The Higgs boson mass dependence of the isolation efficiency is summarized in Figure 6.10 for each type of lepton pair.

\begin{tabular}{c|ccc}
\hline \hline Type & Data $Z \rightarrow \ell \ell$ & Monte Carlo $Z \rightarrow \ell \ell$ & Scale Factor \\
\hline CEM & $0.823 \pm 0.006$ & $0.833 \pm 0.002$ & $0.988 \pm 0.007$ \\
CMUP & $0.916 \pm 0.007$ & $0.937 \pm 0.002$ & $0.978 \pm 0.008$ \\
CMX & $0.929 \pm 0.007$ & $0.930 \pm 0.002$ & $0.999 \pm 0.008$ \\
\hline \hline
\end{tabular}

Table 6.2: Isolation cut efficiencies for Monte Carlo $Z \rightarrow \ell \ell$ and data for each lepton type. 

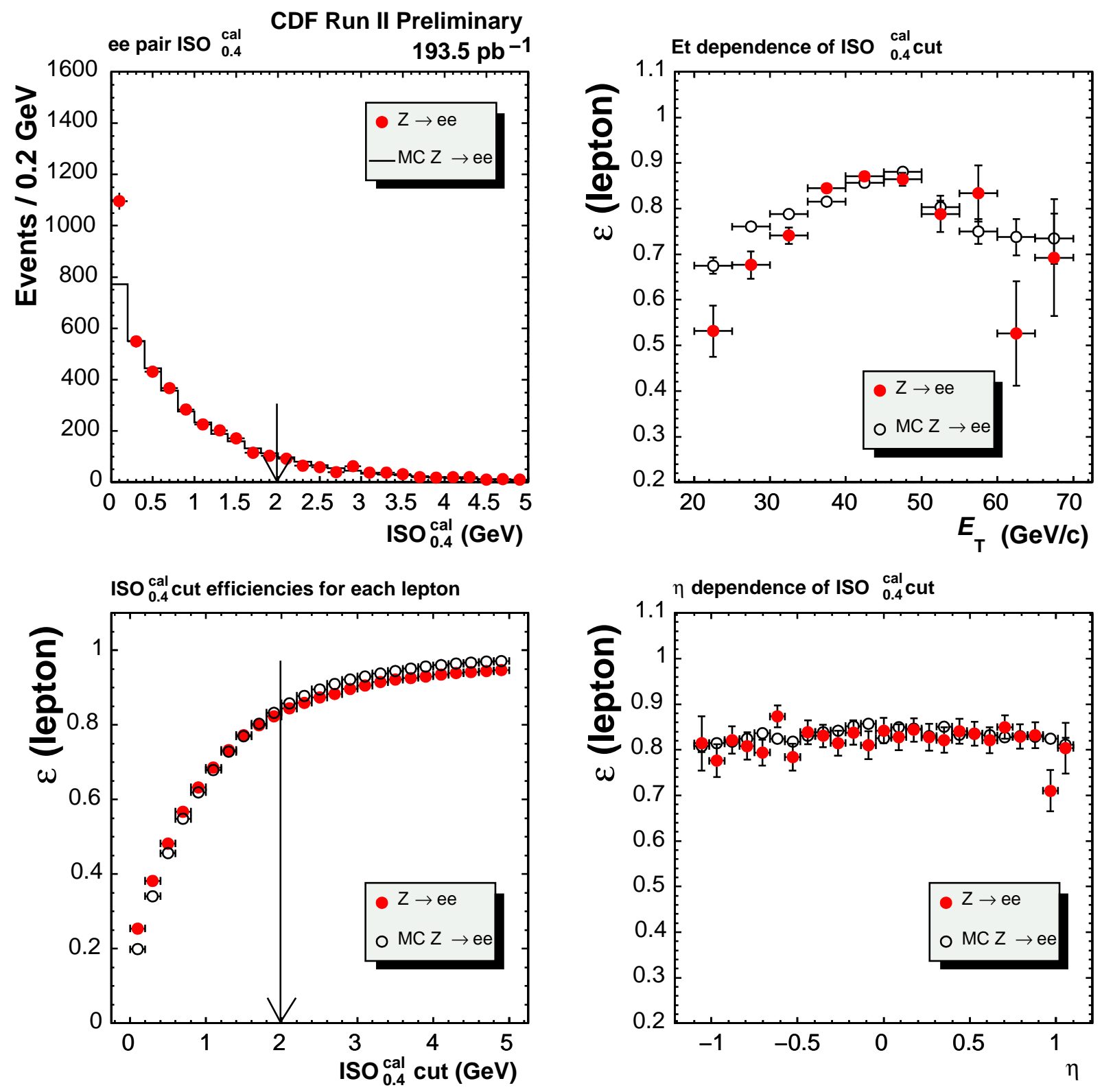

Figure 6.5: Comparison between Monte Carlo $Z \rightarrow e e$ and data one. Upper left plot is raw isolation distribution, upper right one is electron $E_{T}$ dependence, lower left one is the efficiency vs cut value, and lower right one is $\eta$ dependence of the efficiency. 

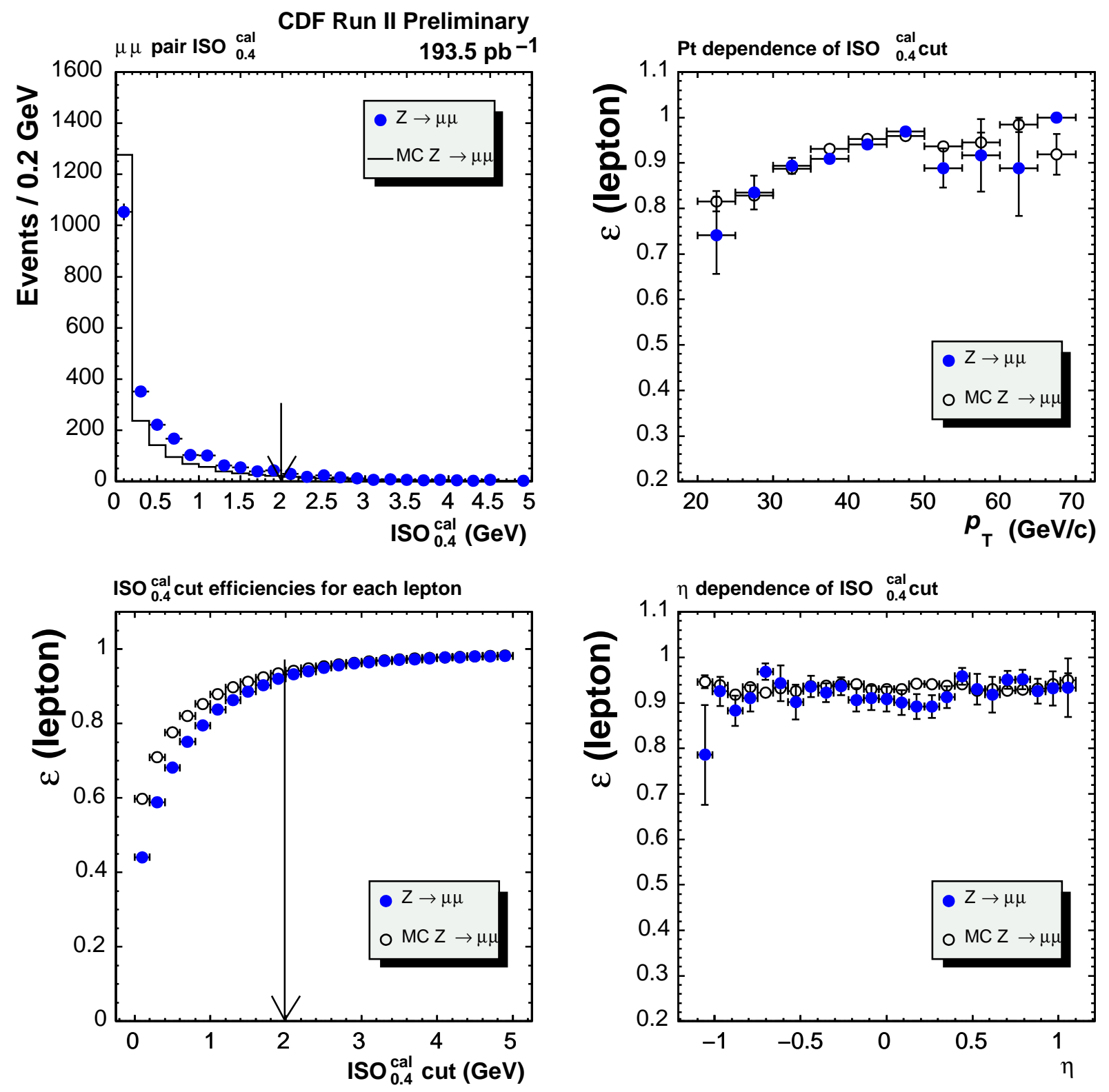

Figure 6.6: Comparison between Monte Carlo $Z \rightarrow \mu \mu$ and data one. Upper left plot is raw isolation distribution, upper right one is electron $E_{T}$ dependence, lower left one is the efficiency vs cut value, and lower right one is $\eta$ dependence of the efficiency. 

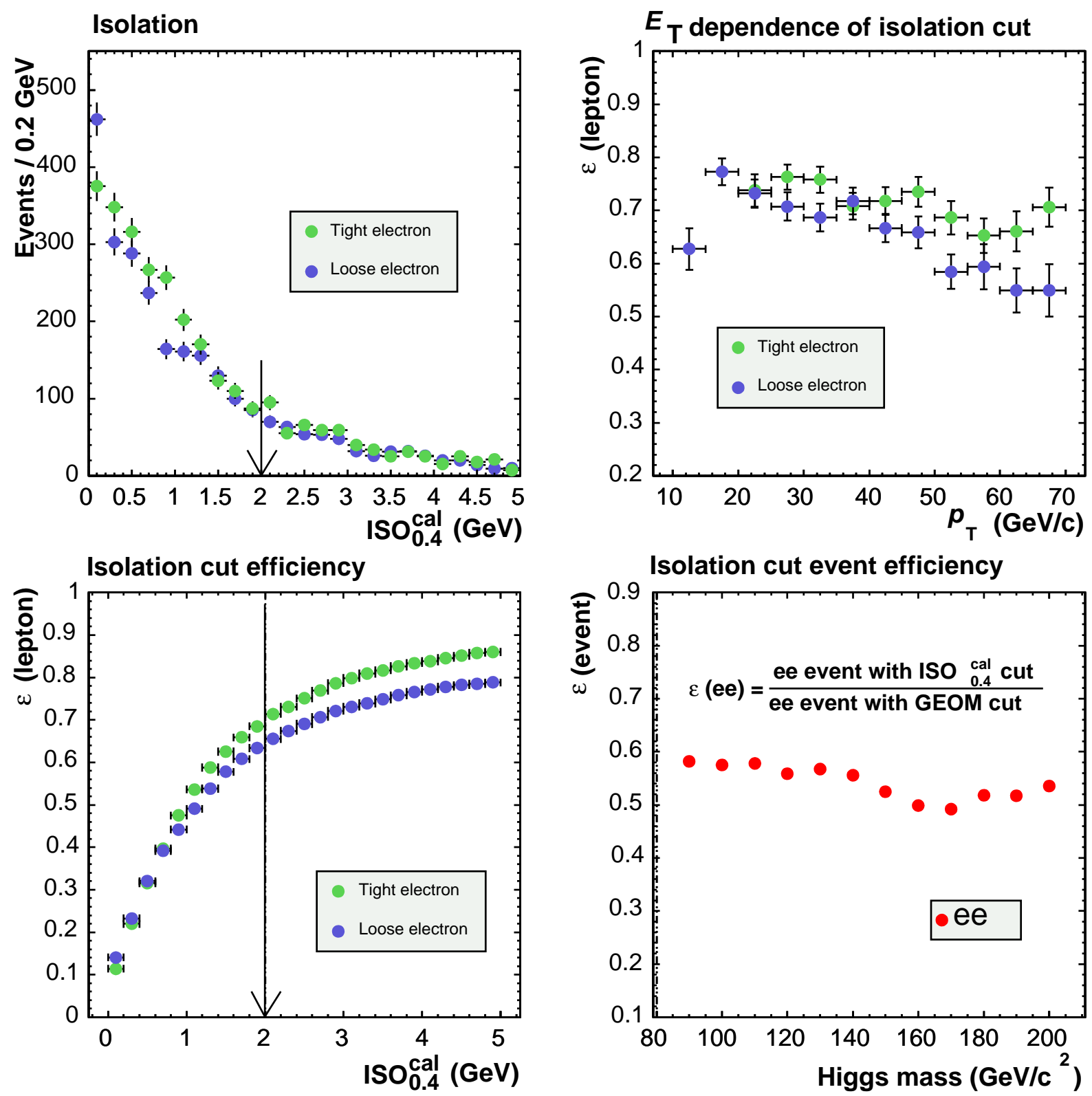

Figure 6.7: The lepton-isolation features of signal Monte Carlo sample. The Higgs boson mass is $160 \mathrm{GeV} / c^{2}$ and the event type is dielectron. Upper left plot is raw $I S O_{0.4}^{\text {cal }}$ distribution, upper right plot is $E_{T}$ dependence of isolation cut efficiency, lower left plot is the isolation cut efficiency as a function of the cut value, and lower right is the cut efficiency as a function of the Higgs boson mass. 

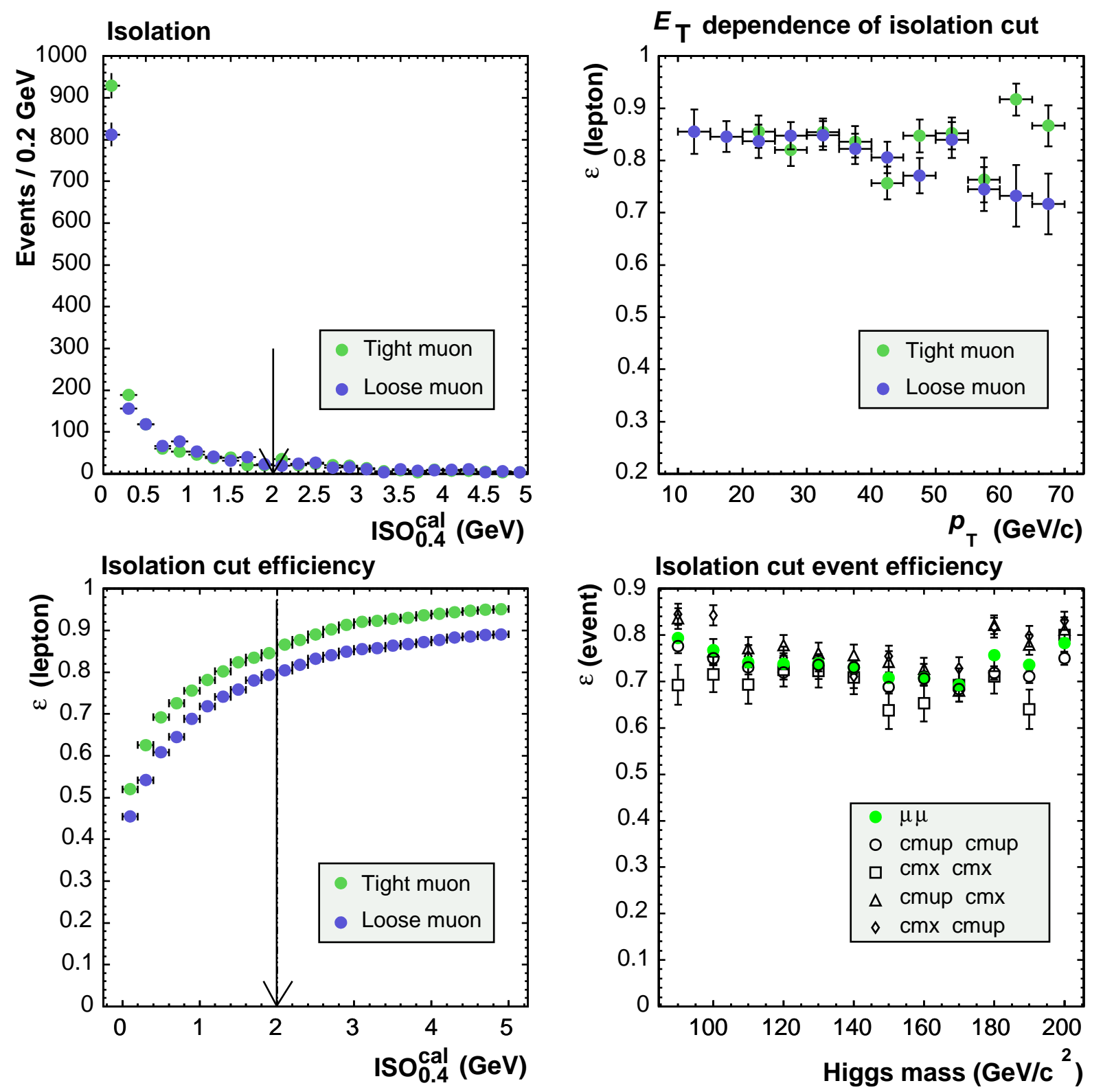

Figure 6.8: The lepton-isolation features of signal Monte Carlo sample. The Higgs boson mass is $160 \mathrm{GeV} / c^{2}$ and the event type is dimuon. Upper left plot is raw $I S O_{0.4}^{\text {cal }}$ distribution, upper right plot is $E_{T}$ dependence of isolation cut efficiency, lower left plot is the isolation cut efficiency as a function of the cut value, and lower right is the cut efficiency as a function of the Higgs boson mass. 

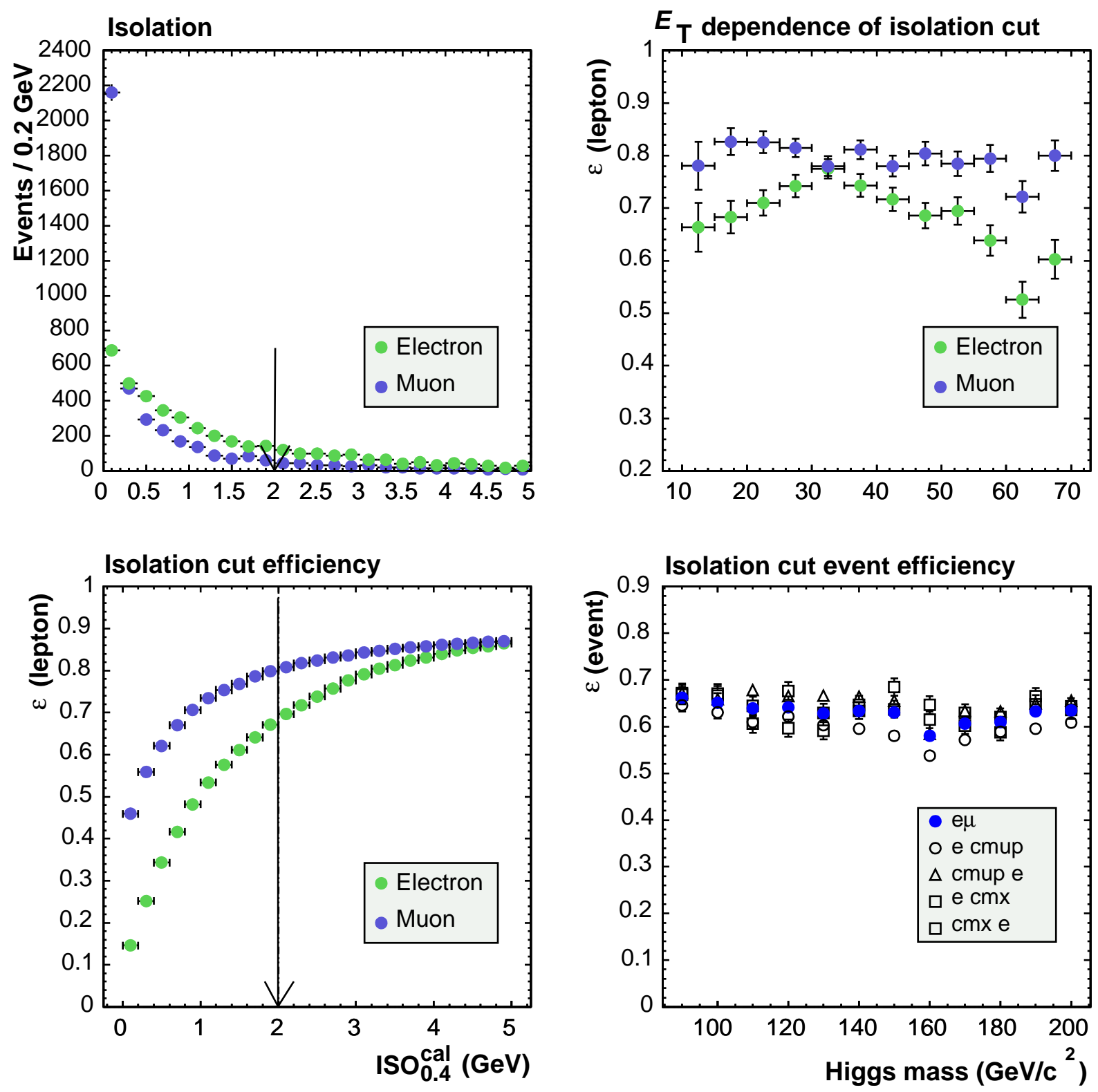

Figure 6.9: The lepton-isolation features of signal Monte Carlo sample. The Higgs boson mass is $160 \mathrm{GeV} / c^{2}$ and the event type is $e \mu$. Upper left plot is raw $I S O_{0.4}^{\text {cal }}$ distribution, upper right plot is $E_{T}$ dependence of isolation cut efficiency, lower left plot is the isolation cut efficiency as a function of the cut value, and lower right is the cut efficiency as a function of the Higgs boson mass. 

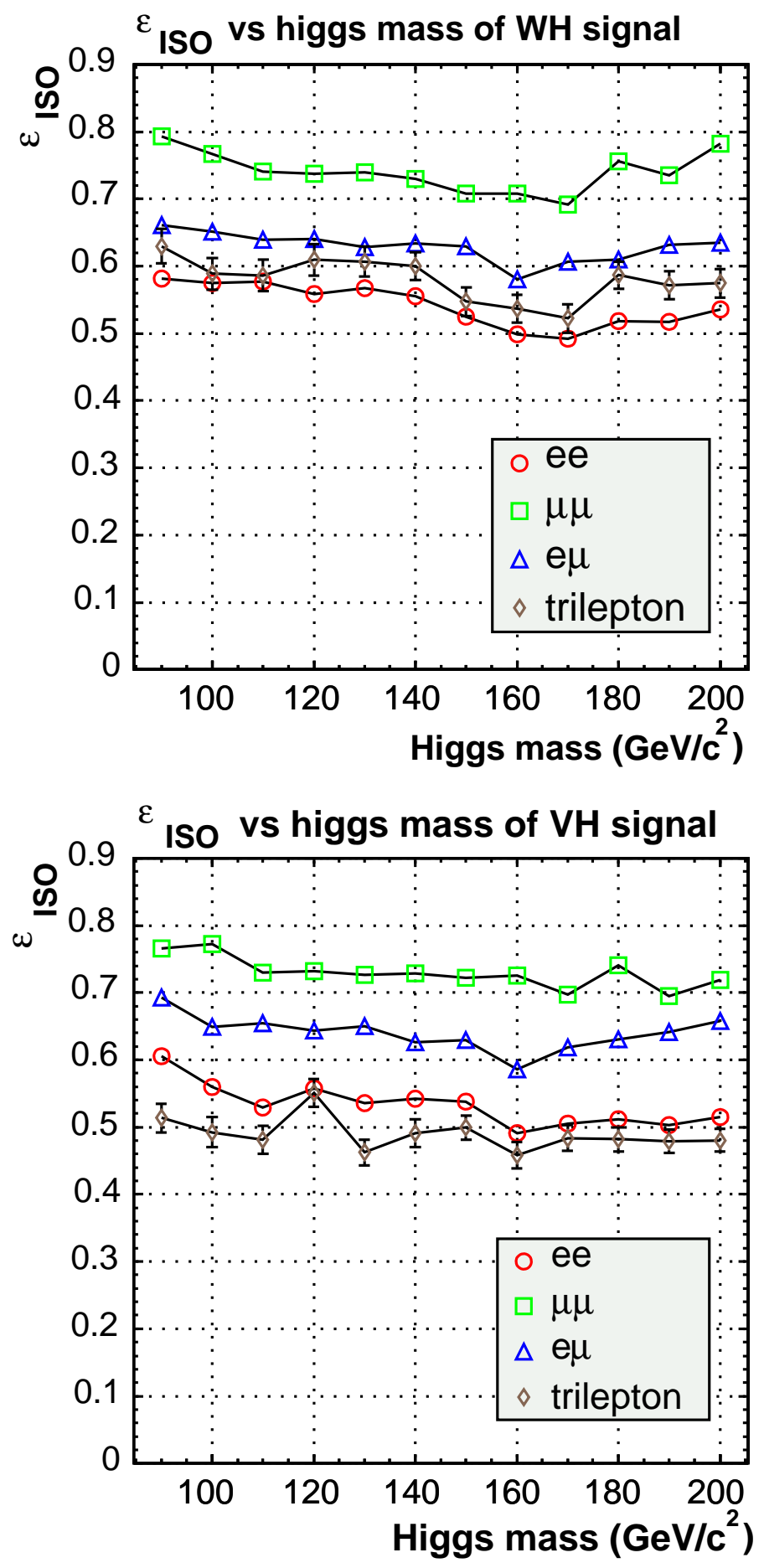

Figure 6.10: Upper (Lower) plot is the isolation cut efficiencies as a function of the Higgs boson mass for $W H(W H+Z H)$ production. The circle point is dielectron channel, square one is dimuon channel, triangle one is $e \mu$ channel, and diamond one is trilepton channel. 


\subsection{Lepton Identification Efficiency}

We also use scale factors and MC efficiencies to estimate the lepton identification efficiency. We estimate the scale factors by comparing the lepton identification efficiency in $Z \rightarrow \ell \ell$ between the MC and data after applying the geometrical, kinematical, and isolation cuts. We calculate the efficiency of lepton identification cuts by Equation (6.2) and (6.3) in the same way as that of the isolation efficiency calculation. Tables 6.3 and 6.4 show the efficiencies for each lepton identification cut and scale factors. Here, the efficiency of each cut is obtained by applying only that cut to the 2nd $Z$-leg. Also, for the dimuon case, CMX-CMUP, for example, means that the CMX is the first leg and the CMUP is the unbiased leg which we are using for the efficiency estimation. Figure 6.11 shows the lepton identification cut efficiency as a function of the Higgs boson mass. The Higgs boson mass dependence of the efficiency is small for each type of lepton pair.

\subsection{Dilepton Selection Efficiency}

In the dilepton selection cuts (Table 3.4) we require a veto of the known dilepton resonance and apply the quality cuts of dilepton events to select generic like-sign dilepton events. The efficiency of the dilepton cuts except the $z_{0}$ separation cut is estimated from the MC samples. We estimate the scale factor for the $z_{0}$ separation cut between a lepton pair using $Z \rightarrow \ell \ell$ data and the MC samples. The $z_{0}^{\ell 1}-z_{0}^{\ell 2}$ distributions are shown in Figure 6.12 for the data and MC samples. The efficiencies of $z_{0}$ separation cut are listed in Table 6.5 for each type of lepton. The Higgs boson mass dependence of the dilepton selection cut efficiencies are shown in Figure 6.13. The acceptance of ee and $\mu \mu$ events in the $W H+Z H$ signal sample is less than that of $e \mu$ events since there are decay modes associated with $Z$ bosons producing opposite-sign dilepton pairs, and they are suppressed by the $Z$ veto cut and like-sign requirement in this analysis. 


\begin{tabular}{c|ccc}
\hline \hline Cut & Data $Z \rightarrow e e$ & Monte Carlo $Z \rightarrow e e$ & Scale Factor \\
\hline HAD/EM & $0.993 \pm 0.001$ & $0.989 \pm 0.001$ & $1.00 \pm 0.001$ \\
$L_{\text {shr }}$ & $0.991 \pm 0.001$ & $0.974 \pm 0.001$ & $1.02 \pm 0.001$ \\
$E / p$ & $0.932 \pm 0.003$ & $0.939 \pm 0.001$ & $0.993 \pm 0.003$ \\
$\chi_{\text {strip }}^{2}$ & $0.993 \pm 0.001$ & $0.998 \pm 0.001$ & $0.995 \pm 0.001$ \\
$\left|\Delta z_{\text {CES }}\right|$ & $0.994 \pm 0.001$ & $0.996 \pm 0.001$ & $0.997 \pm 0.001$ \\
$Q \times \Delta x_{\text {CES }}$ & $0.980 \pm 0.002$ & $0.991 \pm 0.001$ & $0.989 \pm 0.002$ \\
Track quality & $0.974 \pm 0.002$ & $0.992 \pm 0.001$ & $0.982 \pm 0.002$ \\
$\left|z_{0}-z_{\text {vtx }}\right|$ & $0.986 \pm 0.001$ & $0.992 \pm 0.001$ & $0.994 \pm 0.001$ \\
$|d 0|$ & $0.971 \pm 0.002$ & $0.984 \pm 0.001$ & $0.986 \pm 0.002$ \\
Conversion veto & $0.943 \pm 0.003$ & $0.967 \pm 0.001$ & $0.976 \pm 0.003$ \\
\hline Total & $0.822 \pm 0.005$ & $0.862 \pm 0.001$ & $0.954 \pm 0.006$ \\
\hline \hline
\end{tabular}

Table 6.3: Electron identification cut efficiencies for data $Z \rightarrow e e$ and the Monte Carlo samples. The scale factors (Data/Monte Carlo) are listed in the rightmost column. 


\begin{tabular}{|c|c|c|c|}
\hline Cut & \multicolumn{2}{|c|}{ Data $Z \rightarrow \mu \mu \quad$ Monte Carlo $Z \rightarrow e e$} & Scale Factor \\
\hline \multicolumn{4}{|c|}{ CMUP - CMUP } \\
\hline EM & $0.968 \pm 0.004$ & $4 \quad 0.959 \pm 0.001$ & $1.00 \pm 0.00$ \\
\hline HAD & $0.981 \pm 0.003$ & $0.977 \pm 0.001$ & $1.01 \pm 0.00$ \\
\hline$r \times \Delta \phi$ & $0.998 \pm 0.001$ & $0.999 \pm 0.001$ & $1.00 \pm 0.00$ \\
\hline Track Quality & $0.977 \pm 0.003$ & $0.996 \pm 0.001$ & $0.982 \pm 0.003$ \\
\hline$\left|z_{0}-z_{\mathrm{vtx}}\right|$ & $0.995 \pm 0.002$ & $0.998 \pm 0.001$ & $0.997 \pm 0.002$ \\
\hline$\left|d_{0}\right|$ & $0.996 \pm 0.001$ & $0.998 \pm 0.001$ & $0.998 \pm 0.001$ \\
\hline Total & $0.923 \pm 0.006$ & $6 \quad 0.930 \pm 0.001$ & $0.993 \pm 0.007$ \\
\hline \multicolumn{4}{|c|}{ CMX - CMUP } \\
\hline EM & $0.944 \pm 0.010$ & $0 \quad 0.930 \pm 0.002$ & $1.02 \pm 0.01$ \\
\hline HAD & $0.979 \pm 0.006$ & $0.955 \pm 0.002$ & $1.02 \pm 0.01$ \\
\hline$r \times \Delta \phi$ & $0.996 \pm 0.003$ & $0.999 \pm 0.001$ & $0.997 \pm 0.003$ \\
\hline Track Quality & $0.958 \pm 0.009$ & $0.994 \pm 0.001$ & $0.965 \pm 0.009$ \\
\hline$\left|z_{0}-z_{\mathrm{vtx}}\right|$ & $0.983 \pm 0.006$ & $0.995 \pm 0.001$ & $0.988 \pm 0.006$ \\
\hline$\left|d_{0}\right|$ & $0.993 \pm 0.004$ & $0.996 \pm 0.001$ & $0.997 \pm 0.004$ \\
\hline Total & $0.868 \pm 0.015$ & $5 \quad 0.873 \pm 0.003$ & $0.993 \pm 0.017$ \\
\hline \multicolumn{4}{|c|}{ CMX - CMX } \\
\hline EM & $0.954 \pm 0.009$ & $\begin{array}{ll}9 & 0.962 \pm 0.002\end{array}$ & $0.992 \pm 0.009$ \\
\hline HAD & $0.983 \pm 0.005$ & $0.970 \pm 0.001$ & $1.01 \pm 0.01$ \\
\hline$r \times \Delta \phi$ & $0.974 \pm 0.007$ & $0.998 \pm 0.001$ & $0.976 \pm 0.007$ \\
\hline Track Quality & $0.977 \pm 0.006$ & $0.993 \pm 0.001$ & $0.984 \pm 0.006$ \\
\hline$\left|z_{0}-z_{\mathrm{vtx}}\right|$ & $0.991 \pm 0.004$ & $0.998 \pm 0.001$ & $0.993 \pm 0.004$ \\
\hline$\left|d_{0}\right|$ & $0.995 \pm 0.003$ & $0.998 \pm 0.001$ & $0.997 \pm 0.003$ \\
\hline Total & $0.884 \pm 0.013$ & $3 \quad 0.922 \pm 0.002$ & $0.960 \pm 0.015$ \\
\hline \multicolumn{4}{|c|}{ CMUP - CMX } \\
\hline EM & $0.961 \pm 0.008$ & $8 \quad 0.927 \pm 0.002$ & $1.04 \pm 0.01$ \\
\hline HAD & $0.967 \pm 0.008$ & $0.937 \pm 0.002$ & $1.03 \pm 0.01$ \\
\hline$r \times \Delta \phi$ & $0.954 \pm 0.009$ & $0.995 \pm 0.001$ & $0.959 \pm 0.009$ \\
\hline Track Quality & $0.971 \pm 0.007$ & $0.987 \pm 0.001$ & $0.984 \pm 0.007$ \\
\hline$\left|z_{0}-z_{\mathrm{vtx}}\right|$ & $0.987 \pm 0.005$ & $0.995 \pm 0.001$ & $0.992 \pm 0.005$ \\
\hline$\left|d_{0}\right|$ & $0.986 \pm 0.005$ & $0.998 \pm 0.001$ & $0.989 \pm 0.005$ \\
\hline Total & $0.838 \pm 0.016$ & $0.846 \pm 0.003$ & $0.991 \pm 0.019$ \\
\hline & Muon type & Combined scale factor & \\
\hline & CMUP & $0.993 \pm 0.007$ & \\
\hline & CMX & $0.972 \pm 0.015$ & \\
\hline
\end{tabular}

Table 6.4: Muon identification cut efficiencies in $Z \rightarrow \mu \mu$ for data and the Monte Carlo samples for each dimuon combination (CMUP-CMUP, CMX-CMUP, CMX-CMX, and CMUP-CMX). The scale factors (Data/Monte Carlo) are listed in the rightmost column. The combined value is the weighted average value. 


\begin{tabular}{c|ccc}
\hline \hline Type & Data $Z \rightarrow \ell \ell$ & Monte Carlo $Z \rightarrow \ell \ell$ & Scale Factor \\
\hline CEM & $0.992 \pm 0.002$ & $0.997 \pm 0.001$ & $0.995 \pm 0.002$ \\
CMUP & $0.990 \pm 0.003$ & $0.997 \pm 0.001$ & $0.993 \pm 0.003$ \\
CMX & $0.998 \pm 0.002$ & $0.997 \pm 0.001$ & $1.00 \pm 0.00$ \\
\hline \hline
\end{tabular}

Table 6.5: $z_{0}^{l 1}-z_{0}^{l 2}$ cut efficiencies for data $Z \rightarrow \ell \ell$ and Monte Carlo for each lepton type. The scale factor (Data/Monte Carlo) are listed in right row. 

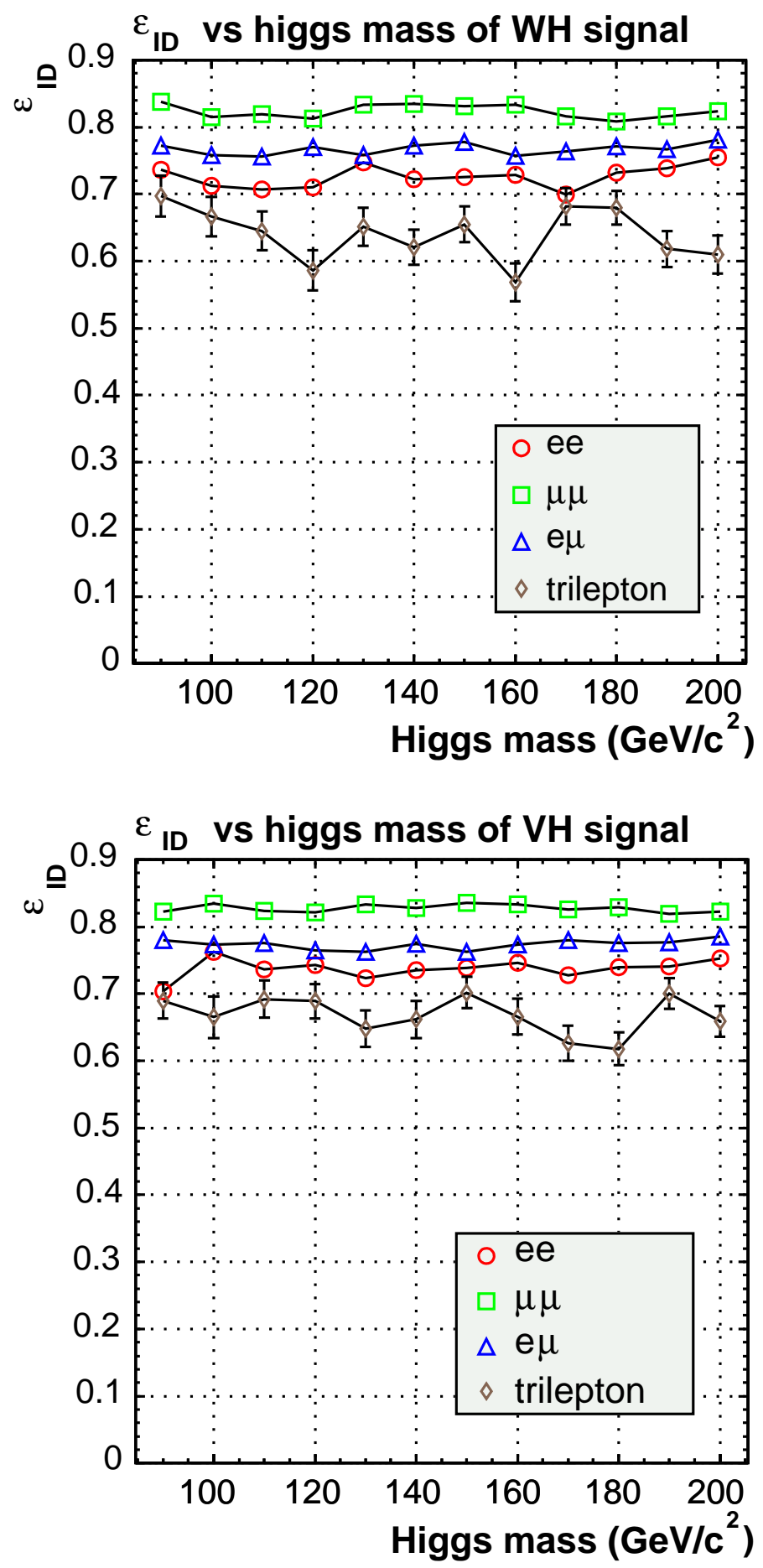

Figure 6.11: Upper (Lower) plot shows the isolation cut efficiencies as a function of the Higgs boson mass for the $W H(W H+Z H)$ production. Circles, squares, triangles, and diamonds correspond to the dielectron channel, dimuon channel, $e \mu$ channel, and trilepton channel respectively. 

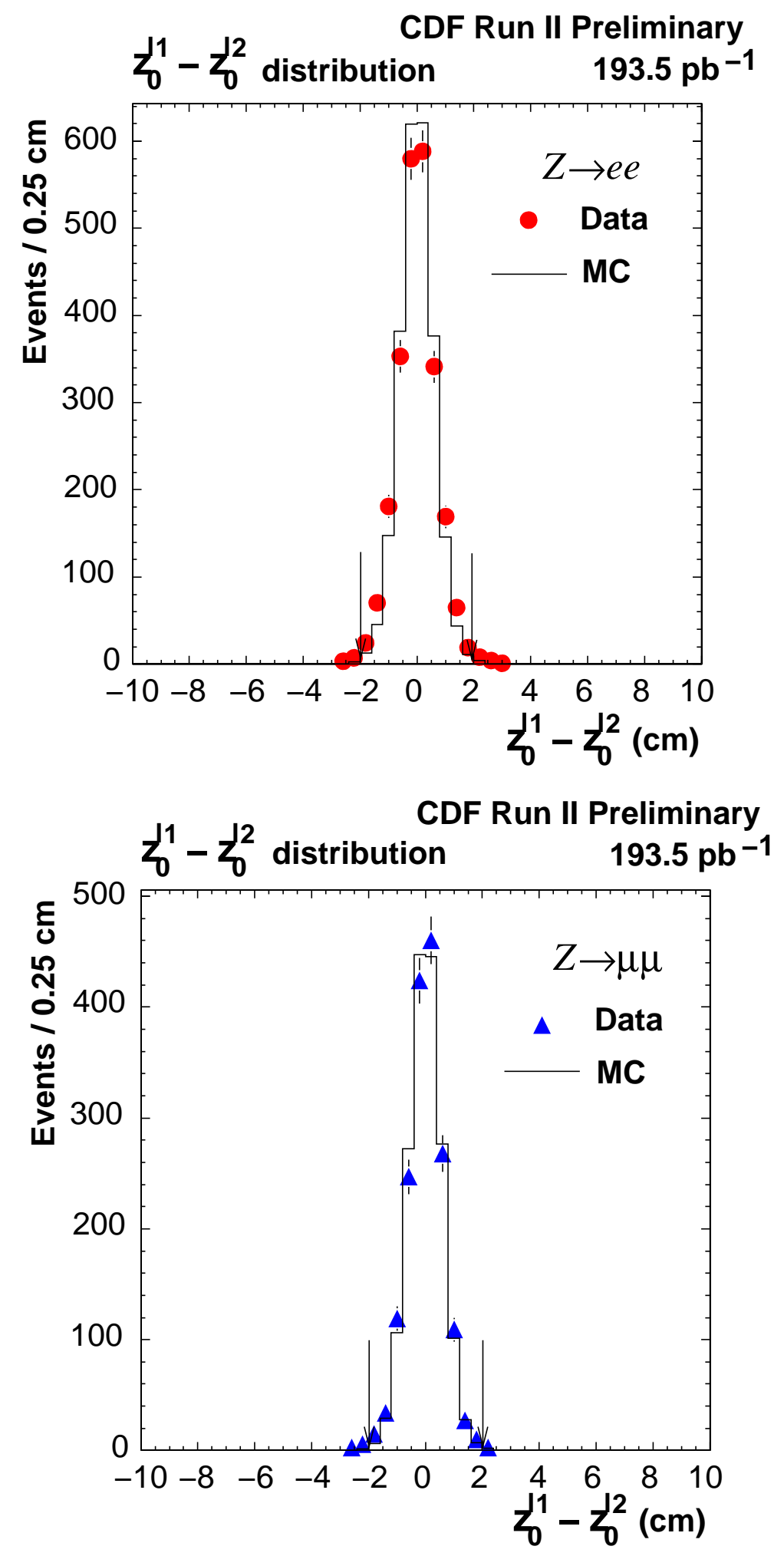

Figure 6.12: $\left|z_{0}^{l 1}-z_{0}^{l 2}\right|$ distributions of $Z \rightarrow \ell \ell$ events. 

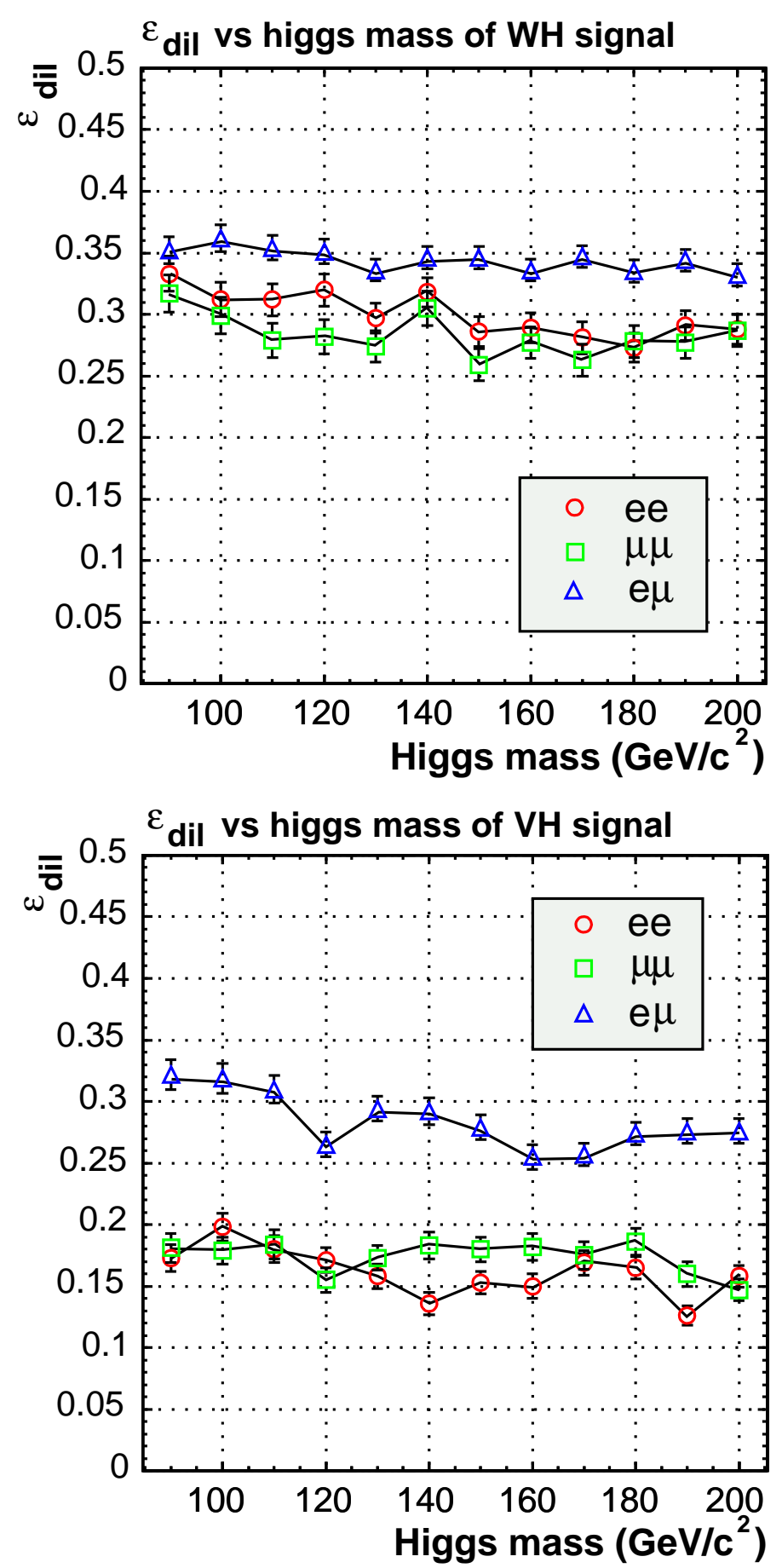

Figure 6.13: Upper (Lower) plot is dilepton selection cuts efficiencies as a function of the Higgs boson mass for $W H(W H+Z H)$ production. The circle point is dielectron channel, square one is dimuon channel, and triangle one is $e \mu$ channel 


\begin{tabular}{|c|c|c|c|}
\hline Trigger name & Level-1 & Level-2 & Level-3 \\
\hline ELECTRON_CENTRAL_18 & $\begin{array}{c}\text { L1_CEM8_PT8 } \\
\text { CEM } E_{T}>8 \mathrm{GeV}, \\
\text { XFT } p_{T}>8 \mathrm{GeV}\end{array}$ & $\begin{array}{c}\text { L2_CEM16_PT8 } \\
\text { CEM } E_{T}>16 \mathrm{GeV}, \\
\text { XFT } p_{T}>8 \mathrm{GeV}\end{array}$ & $\begin{array}{c}\text { L3_ELECTRON_CENTRAL_18 } \\
\text { CEM } E_{T}>18 \mathrm{GeV} \\
\text { XFT } p_{T}>9 \mathrm{GeV}\end{array}$ \\
\hline W_NOTRACK & $\begin{array}{c}\text { L1_EM8_l\&_MET15 } \\
\text { EM } E_{T}>8 \mathrm{GeV}, \\
E_{T}>15 \mathrm{GeV}\end{array}$ & $\begin{array}{l}\text { L2_CEM16_L1_MET15 } \\
\text { EM } E_{T}>20 \mathrm{GeV}\end{array}$ & $\begin{array}{c}\text { L3_W_NOTRACK_MET25 } \\
\text { CEM } E_{T}>25 \mathrm{GeV}, \\
E_{T}>15 \mathrm{GeV}\end{array}$ \\
\hline W_NOTRACK_NO_L2 & $\begin{array}{c}\text { L1_EM8_\&_MET15 } \\
\text { EM } E_{T}>8 \mathrm{GeV} \\
\mathbb{E}_{T}>15 \mathrm{GeV}\end{array}$ & $\begin{array}{c}\text { auto accept } \\
\text { Pre-scale } 50\end{array}$ & $\begin{array}{c}\text { L3_W_NOTRACK_MET25 } \\
\text { CEM } E_{T}>25 \mathrm{GeV} \\
\quad E_{T}>15 \mathrm{GeV}\end{array}$ \\
\hline ELECTRON_CENTRAL_8 & $\begin{array}{l}\text { L1_CEM8_PT8 } \\
\text { CEM } E_{T}>8 \mathrm{GeV} \\
\mathrm{XFT} p_{T}>8 \mathrm{GeV}\end{array}$ & $\begin{array}{l}\text { 50_L1_CEM8_0T8_CES2_DPS } \\
\text { Max Pre-scale }=10 \\
\text { CES } E_{T}>2 \mathrm{GeV}\end{array}$ & $\begin{array}{c}\text { L3_ELECTRON_CENTRAL_8_PT8 } \\
\text { CEM } E_{T}>8 \mathrm{GeV} \\
\text { COT } p_{T}>8 \mathrm{GeV}\end{array}$ \\
\hline
\end{tabular}

Table 6.6: The requirements for the high $p_{T}$ electron trigger and the backup triggers.

\subsection{Topological Cut Efficiency}

The topological cuts, $p_{T 2}$ and $p_{T 12}$ cuts are completely kinematical cuts to the like-sign dilepton system. We estimate the efficiencies using the MC samples. As described before the second leg $p_{T}$ depends on the Higgs boson mass. We expect that the topological cut efficiency also depends on the Higgs boson mass. Figure 6.14 shows the topological cut efficiencies for the $W H$ and $W H+Z H$ signal as a function of the Higgs boson mass, where the trilepton category has been merged into the dilepton category based on the types for the leading two leptons.

\subsection{Trigger Efficiency and Other Scale Factors}

We referred to the trigger efficiency of ELECTRON_CENTRAL_18 in [79] and that of MUON_CMUP18 and MUON_CMX18 in [80].

\subsubsection{High $p_{T}$ Electron Trigger Efficiency}

In order to estimate the trigger efficiency used in this analysis, we use the so-called "backup" triggers. The backup triggers consist of the almost same trigger requirements except the quantity that should be estimated. Each trigger path has been tested separately and the correspondent efficiency has been calculated at each step [80].

The requirements for the high $p_{T}$ electron trigger and the backup triggers are listed in Table 6.6. The XFT and CT tracking efficiencies have been calculated using the W_NOTRACK trigger, which demands the same calorimeter requirements used for the high 
$p_{T}$ electron trigger, but does not require tracks associated with the EM clusters. The track trigger efficiencies thus can be measured with events coming from this trigger. In order to select the $W \rightarrow e \nu$ events, we apply the baseline $W$ selection cuts [79] to both triggers. The L1_XFT_PT8 trigger efficiency is measured by counting the $W$ candidate events passing the L1_XFT_PT8 trigger:

$$
\varepsilon(\text { L1_XFT_PT8 })=\frac{\# \text { of } W \text { candidates passing L1_XFT_PT8 }}{\# \text { of } W \text { candidates }}
$$

The efficiency curve is fitted as a function of $p_{T}$, and the resulting trigger efficiency is almost 96.6\%. The Level-3 tracking efficiency is measured by requiring the L1_CEM8_PT8 and L2_CEM16_PT8 bits set and counting the $W$ events with the Level-3 Electron 18 bit set:

$$
\varepsilon(\text { L3_PT_9 })=\frac{\# \text { of } W \text { candidates passing L1_CEM8_PT8 \& L2_CEM16_PT8 \& L3_CEM18_PT9 }}{\# \text { of } W \text { candidates passing L1_CEM8_PT8 \& L2_CEM16_PT8 }}
$$

The tracking efficiency is almost 99\%. The track trigger efficiency is independent of $p_{T}$ in the region of $p_{T}>10 \mathrm{GeV}$, but dependent on $\eta$ for both the Level-1 and Level-3 triggers as shown in Figure 6.15. The calorimeter trigger efficiency at Level-1 has been calculated using the high $p_{T}$ inclusive muon sample which requires one tight $\mathrm{CMU}$ or CMX muon without an isolation cut.

We select events which have CEM activity compatible with the L1_EM8 trigger by combining the calorimeter tower into the trigger tower geometry. The trigger efficiency is estimated by counting the events if the selected events fire the L1_EM8 trigger bit. The Level-2 calorimeter cluster trigger efficiency has been estimated using the W_NOTRACK_NOL2 trigger, which is the same as the W_NOTRACK trigger, but without any requirement at Level-2 (Level-2 Auto Accept). In order to get high purity electron sample, some quality cuts [79] are applied, but $E_{T}$ cut lowered to $18 \mathrm{GeV}$ and an isolation cut $I S O_{0.4}^{\text {cal }}$ less than $4 \mathrm{GeV}$. The $E_{T}$ distribution of electron candidates and the resulting L2_CEM16 trigger efficiency are shown in Figure 6.15. The efficiency curve is fitted as a function of $E_{T}$ :

$$
\varepsilon\left(\mathrm{L} 2 \_\mathrm{CEM} 16\right)=1-p_{0} \cdot \exp \left(-p_{1} \cdot E_{T}\right) .
$$

The L2_CEM16 trigger fires $96.4 \%$ of electrons around $E_{T}=20 \mathrm{GeV}$ and the efficiency reaches up to $99.1 \%$ at $E_{T}$ larger than $25 \mathrm{GeV}$.

The overall high $p_{T}$ electron trigger efficiency is almost dominated by the tracking trigger efficiency (Level-1 and Level-3) and calorimeter trigger efficiency (Level-2). Finally, the estimated electron trigger efficiency is $\varepsilon_{\text {ele }}=93.1 \%$ for the single electron.

\subsubsection{High $p_{T}$ Muon Trigger Efficiency}

We calculate the trigger efficiency values fro the CMUP and CMX muons using the dimuon candidates that fall in the $Z$ mass window $\left([80-100] \mathrm{GeV} / c^{2}\right)$. There are two methods that can be used for calculating the efficiencies: 
- Method 1: Count the number of existing CMUP (CMX) triggered legs in CMX (CMUP) triggered CMX-CMUP $Z$ candidate events,

- Method 2: Use the same type (CMUP-CMUP or CMX-CMX) $Z$ candidate events.

We apply the first method and follow the procedure as described in section 6.3. We obtain an overall $88.7 \pm 0.7 \%$ for MUON_CMUP18, and $95.4 \pm 0.6 \%$ for MUON_CMX18 integrated over the $200 \mathrm{pb}^{-1}$ data. The values listed here are the combined Level- $1 \times$ Level- $2 \times$ Level- $3 \times$ trigger efficiency. The inefficiencies are driven by the Level-1 triggers from the track trigger (XFT) requirements. The Level-2 trigger is fully efficient for CMX muons (auto accepted) or negligible inefficiency is expected for CMUP muons (8 $\mathrm{GeV} / c p_{T}$ requirements). The Level-3 trigger efficiency is measured to be $99.4 \pm 0.3 \%$ for CMUP muons and to be $99.2 \pm 0.3 \%$ for CMX muons [80]. The trigger efficiency has had slight time dependencies during the data taking. For example, a comparison of CMUP trigger efficiencies after the Level-1 XFT configuration change in October 2002 with a sample taken before December 2003 showed that the efficiency dropped from $90.0 \pm 2.3 \%$ to $86.7 \pm 3.6 \%$ [56], although the two values are consistent within the Binomial errors. And the CMX Level-1 trigger was included a CSX hit requirement in October 2002 as well. The CDF Run I CSX tag efficiency for CMX muons were about $85 \%$, which required coincidence of the two sandwich layers. Dropping the coincidence requirement in Run II, the CSX triggers are expected to be about $99 \%$ efficient [58]. This is also checked by using a sub-sample of the CMUP-CMX $Z$ muons by counting which CMX muon pass the CSX requirement that already passed the other trigger requirements. The efficiency is found to be $\varepsilon_{C S X}=99.3 \pm 0.5 \%$ [57].

\subsubsection{Event Weight for the Difference of Integrated Luminos- ity}

We choose the single lepton trigger efficiency in this analysis since the leading lepton with our high $p_{T}$ cut is expected to be already efficient compared to the 2nd lepton with $p_{T}>6 \mathrm{GeV} / c$. We use $93.1 \pm 7.0 \%$ for ELECTRON_CENTRAL_18, $88.7 \pm 0.7 \%$ for MUON_CMUP18, and $95.4 \pm 0.6$ for MUON_CMX18. It is necessary to consider the difference of integrated luminosity between the CEM/CMUP triggers and the CMX trigger. In order to conveniently handle the CMX part, we introduce a weight $w=L_{X} / L$ for the events in which the leading lepton is a CMX muon, where $L$ is the integrated luminosity of the CEM or CMUP trigger and $L_{X}$ is that of the CMX trigger. This weight is (175.3 $\pm 10.3) /(193.5 \pm 11.4)=0.905$. 


\subsection{Total Detection Efficiency}

The total efficiencies for various Higgs boson masses are listed in Table 6.7 and shown in Figure 6.17. The mass dependence of the acceptance is due to the kinematical and topological cut efficiencies. In the high- (low-) mass region the efficiency is about 1.5 (1.0)\% for the $W H \rightarrow W W^{*} W^{*} \rightarrow$ dilepton (including trilepton). In the case of the $W H+Z H$ signal the efficiency is about $1.0 \%$ at the high-mass region.

CDF Run II Preliminary

\begin{tabular}{c|cccc}
\hline \hline Higgs boson mass $\left(\mathrm{GeV} / c^{2}\right)$ & 90 & 100 & 110 & 120 \\
\hline$W H\left(\times 10^{-2}\right)$ & $1.00 \pm 0.04$ & $1.01 \pm 0.04$ & $1.12 \pm 0.04$ & $1.33 \pm 0.04$ \\
$W H+Z H\left(\times 10^{-2}\right)$ & $0.49 \pm 0.02$ & $0.62 \pm 0.03$ & $0.68 \pm 0.03$ & $0.63 \pm 0.03$ \\
\hline Higgs boson mass $\left(\mathrm{GeV} / c^{2}\right)$ & 130 & 140 & 150 & 160 \\
\hline$W H\left(\times 10^{-2}\right)$ & $1.42 \pm 0.05$ & $1.70 \pm 0.05$ & $1.68 \pm 0.05$ & $1.55 \pm 0.05$ \\
$W H+Z H\left(\times 10^{-2}\right)$ & $0.81 \pm 0.03$ & $0.84 \pm 0.03$ & $0.94 \pm 0.03$ & $0.88 \pm 0.04$ \\
\hline Higgs boson mass $\left(\mathrm{GeV} / c^{2}\right)$ & 170 & 180 & 190 & 200 \\
\hline$W H\left(\times 10^{-2}\right)$ & $1.70 \pm 0.05$ & $1.69 \pm 0.05$ & $1.86 \pm 0.05$ & $1.76 \pm 0.05$ \\
$W H+Z H\left(\times 10^{-2}\right)$ & $0.93 \pm 0.04$ & $1.02 \pm 0.04$ & $0.92 \pm 0.03$ & $0.98 \pm 0.04$ \\
\hline \hline
\end{tabular}

Table 6.7: Total signal acceptance as a function of the Higgs boson mass. The denominator of the acceptance is $W H(W H+Z H) \rightarrow W W W(V V V) \rightarrow$ dilepton or trilepton events.

\subsection{Systematic Uncertainties}

In this section we describe the systematic error related to the signal acceptance. The systematic error of background estimation are described in [77]. We list the sources of systematic error in Table 6.8. We use $160 \mathrm{GeV} / c^{2}$ Higgs boson mass sample as the reference sample. The statistical uncertainty of the MC samples is about $2 \%$ for each lepton pair and $3.7 \%$ for the total dilepton. We make three samples to check the PDF, FSR, and ISR systematic uncertainties. We take relative differences of the geometrical and kinematical acceptance as the systematic error. The central value for the acceptance has been obtained with the CTEQ PDF's, while we generate a MC sample with the MRST PDF. The systematic error due to the FSR and ISR are estimated using two special samples generated with PYTHIA. The first sample is generated with both the QED and QCD ISR's switched off. The second one uses a different parameter setting, so called the Tune B, for the underlying event. The Tune $\mathrm{A}$ is used for the reference PYTHIA sample. The total systematic error is $6.4 \%$. To check the mass dependence we make $110 \mathrm{GeV} / c^{2}$ Higgs boson mass samples and we 
CDF Run II Preliminary

\begin{tabular}{c|ccc|c}
\hline \hline Sources & $e e(\mathbf{\%})$ & $\mu \mu(\mathbf{\%})$ & $e \mu(\mathbf{\%})$ & Dilepton $\mathbf{( \% )}$ \\
\hline Uncertainties of ISO and ID scale factor & 0.20 & 0.15 & 0.28 & 0.37 \\
Uncertainties of trigger efficiency & 1.8 & 0.11 & 1.6 & 2.4 \\
Statistical uncertainties of the MC & 1.7 & 1.5 & 2.4 & 3.7 \\
Uncertainties from PDF & 1.1 & 1.6 & 1.6 & 1.5 \\
Difference of FSR (Tune A $\leftrightarrow$ Tune B) & 4.8 & 4.3 & 1.8 & 3.2 \\
Difference of ISR $(\mathrm{ON} \leftrightarrow$ OFF) & 2.8 & 3.5 & 2.9 & 3.0 \\
\hline Total $\left(160 \mathrm{GeV} / c^{2}\right)$ & & & & 6.4 \\
Total $\left(110 \mathrm{GeV} / c^{2}\right)$ & & & 7.5 \\
\hline \hline
\end{tabular}

Table 6.8: Systematic error sources and errors.

find the total systematic error to be $7.5 \%$. The mass dependence of the systematic error is not large. 

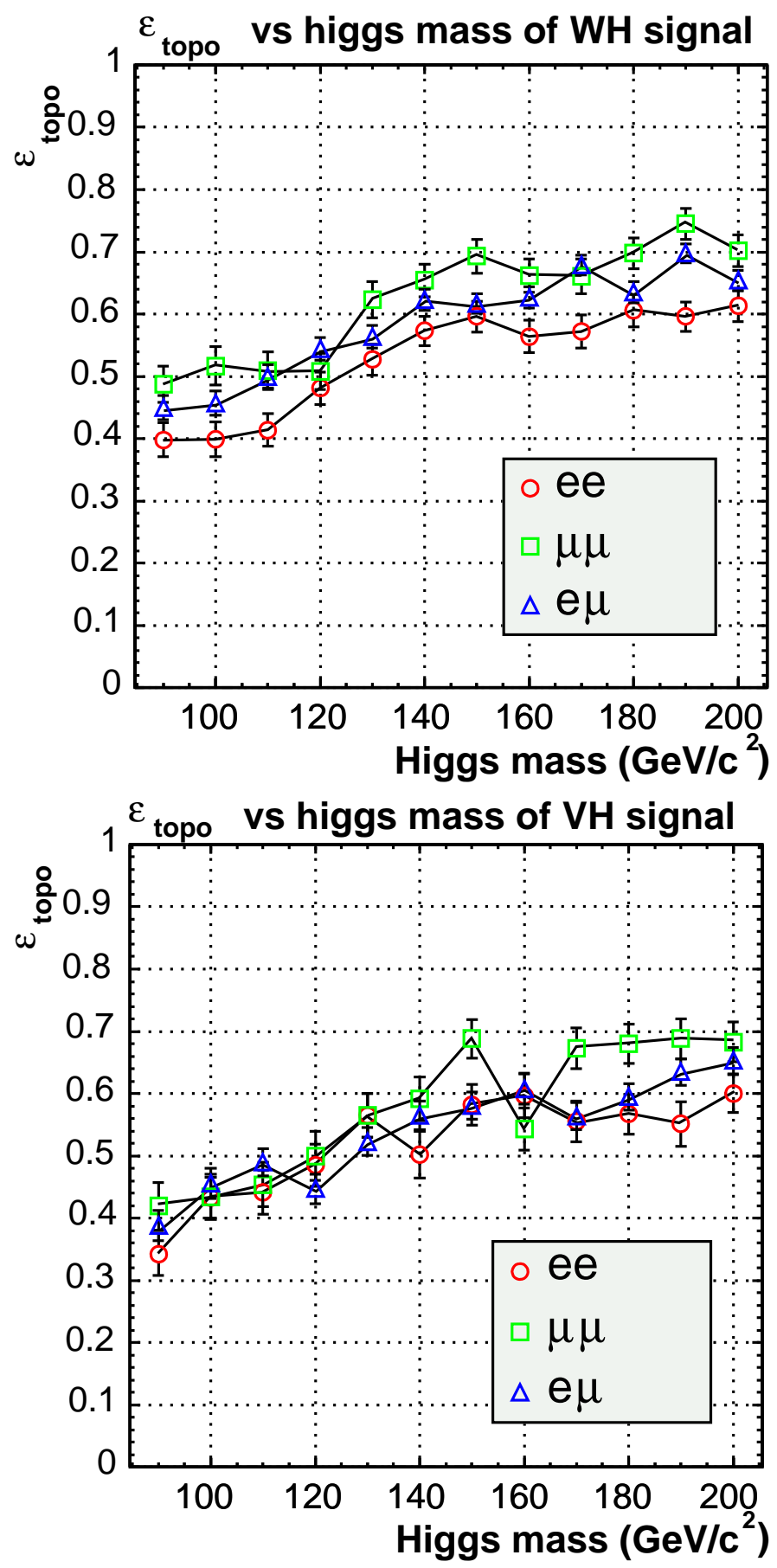

Figure 6.14: Upper (Lower) plot is topological selection cuts efficiencies as a function of the Higgs boson mass for $W H(W H+Z H)$ production. The circle point is dielectron channel, square one is dimuon channel, and triangle one is $e \mu$ channel. 


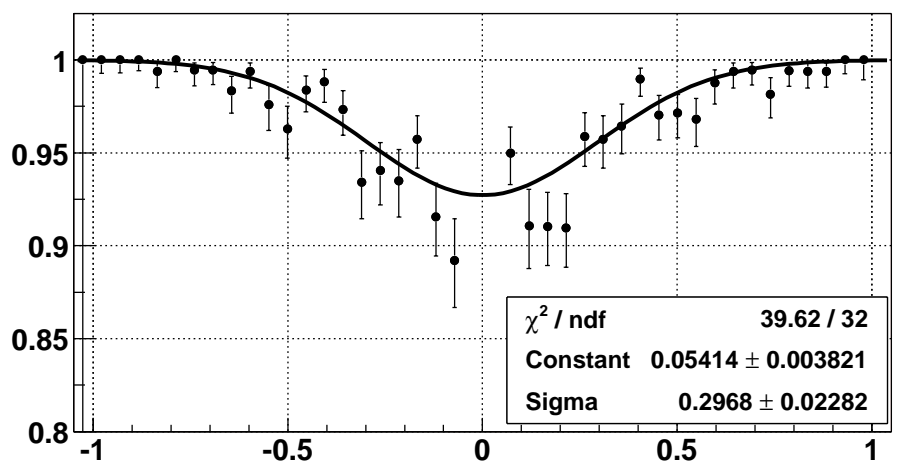

L1 Track Efficiency for W Candidates vs $\eta$

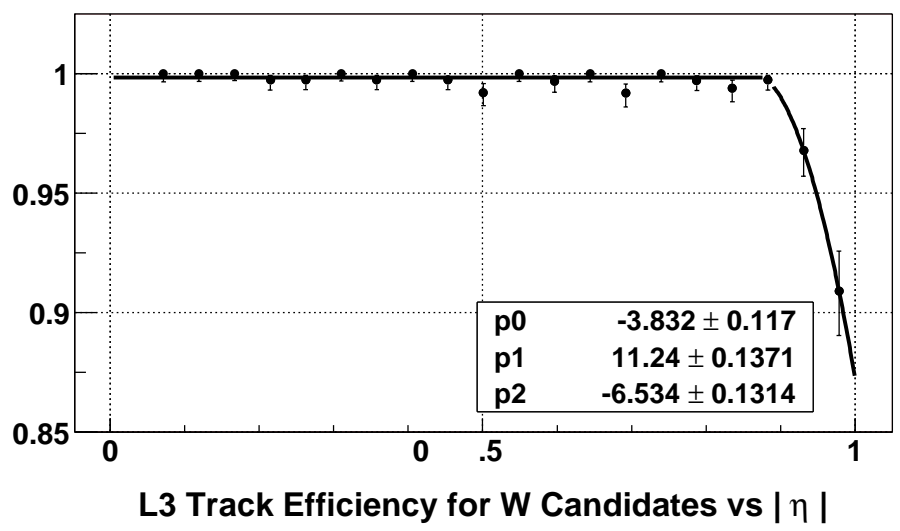

Figure 6.15: Level-1 (top) and Level-3 (bottom) tracking efficiencies as a function of $\eta$ measured by CES.

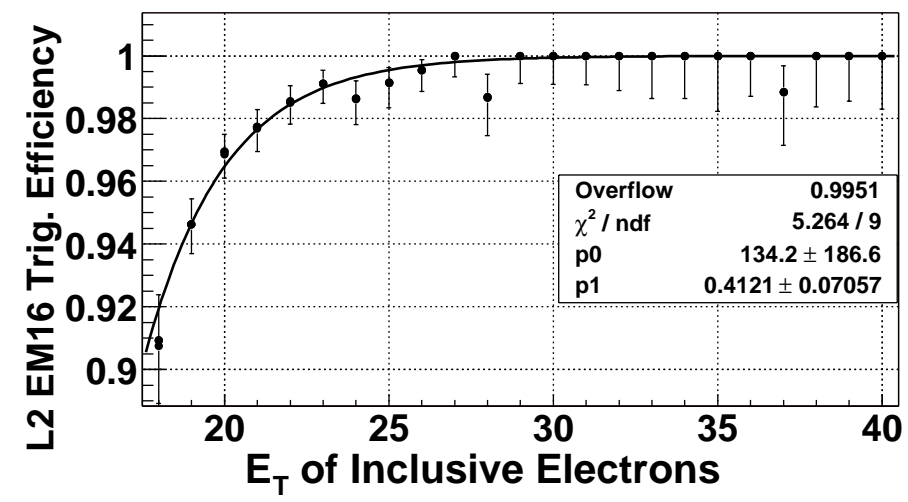

Figure 6.16: L2_CEM16 trigger efficiency as a function of $E_{T}$. 


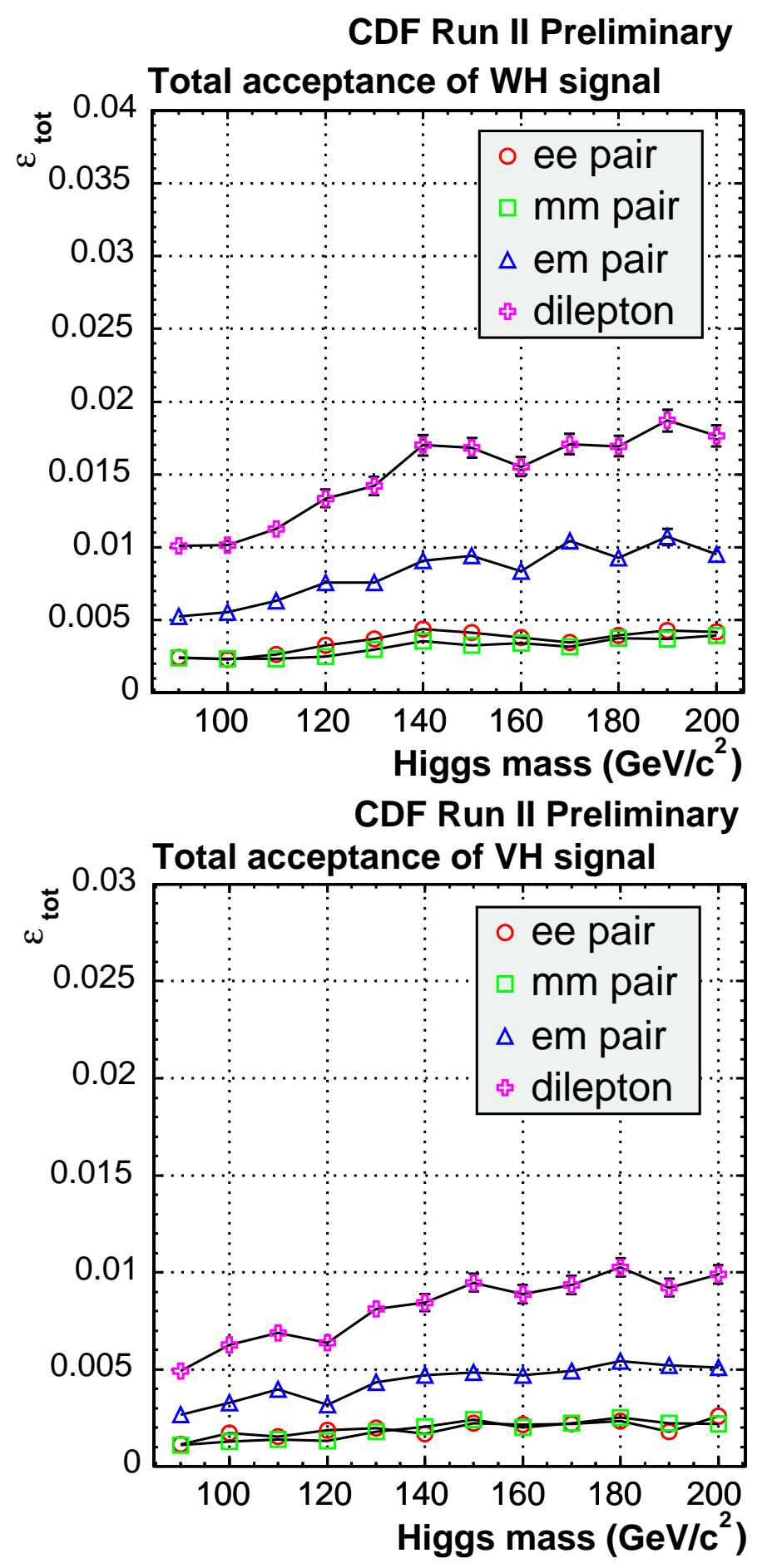

Figure 6.17: Upper (Lower) plot is total signal acceptance as a function of the Higgs boson mass for $W H(W H+Z H)$ production. The circle point is dielectron channel, square one is dimuon channel, triangle one is $e \mu$ channel, and crossed one is the dilepton total (including trilepton events). 


\section{Chapter 7}

\section{Results and Conclusions}

\subsection{Pseudo Experiments}

The pseudo experiment is a mock numeric test. Since we have a number of expected backgrounds and the signal acceptance for the final like-sign dilepton events, we can simulate the observed events and the limit of $W H$ production cross section. Before opening the signal box we need to make pseudo experiments to check whether the final observed limit is reasonable level from the background expectation and the signal acceptance. We make 300 pseudo experiments for each Higgs boson mass point. The signal acceptance and integrated luminosity $(193.5 \pm 11.4 \mathrm{pb})$ is made to fluctuate by Gaussian uncertainties and the number of observed events is given by the Poisson probability with the expected background events. The 95\% C.L. upper limit of signal events is calculated using the Bayesian method with flat prior probability [81]. The cross section limit to the $W H \rightarrow W W W$ decay mode is calculated by the following formula:

$$
\sigma_{95}=\frac{N_{95}}{B r(\geq 2 \text { lepton }) \cdot \varepsilon_{t o t} \cdot L},
$$

where $\sigma_{95}$ is a $95 \%$ C.L. cross section upper limit, $N_{95}$ is a $95 \%$ C.L. upper limit of signal events, and $\operatorname{Br}(\geq 2$ lepton $)$ is a branching ratio of $W W W \rightarrow$ dilepton or trilepton decay mode. Figures 7.1 and Table 7.1 show the results of the pseudo experiments. The expected cross section upper limits are $16.0 \pm 4.7 \mathrm{pb}$ for the Higgs boson mass of $110 \mathrm{GeV} / c^{2}$ and $11.8 \pm 3.8 \mathrm{pb}$ for the Higgs boson mass of $160 \mathrm{GeV} / c^{2}$.

\subsection{Observed 95\% C.L. Cross Section Upper Limit}

We observed no event in the signal box in the $p_{T 2}$ vs $p_{T 12}$ plane. We calculated the $95 \%$ C.L. upper limit on the cross section in the same way as the pseudo experiments. Figure 7.2 showed the observed limit line and the expected cross section limit line 
obtained from the pseudo experiment results. We set the 95\% C.L. upper limit of the cross section $\sigma(W H) \times B r(H \rightarrow W W)$ to be $12 \mathrm{pb}$ for the Higgs boson mass of 110 $\mathrm{GeV} / c^{2}$ and $8 \mathrm{pb}$ for the Higgs boson mass of $160 \mathrm{GeV} / c^{2}$.

\subsection{The Other Direct SM Higgs Boson Search in CDF Run II}

Currently we got the other two preliminary cross section limit for the Standard Model Higgs boson in CDF Run II. The first one is a search for new particle decaying into $b \bar{b}$ and produced in association with $W^{ \pm}$boson in the reaction $W H \rightarrow \ell \nu b \bar{b}$. This search focus on the Standard Model Higgs boson or Technicolor particle decaying into $b \bar{b}$ using approximately $162 \mathrm{pb}^{-1}$ [82]. Events with an electron or muon, missing $\mathbb{E}_{T}$, and two jets, one of them $b$-tagged, are selected. The number of tagged events and the dijet mass distribution are consistent with the Standard Model expectation. The 95\% C.L. upper limit on the production cross section times branching ratio is about $5 \mathrm{fb}$ for the Higgs boson mass $115 \mathrm{GeV}$. The second one is a search for the Standard Model Higgs production and decay in the channel $g g \rightarrow H \rightarrow W W \rightarrow \ell \nu \ell \nu$ [83]. Observing no signal excess, they set a production cross section upper limit as a function of the Higgs boson mass. They employ the fact that the azimuthal angle between the finalstate leptons $\Delta \phi_{\ell \ell}$ is predicted to be smaller on average for the Higgs boson decays than for the background processes. The $95 \%$ C.L. limit of the production cross section times branching ratio is about $6 \mathrm{pb}$ for the Higgs mass $160 \mathrm{GeV} / c^{2}$. Figure 7.3 showed the observed limit line and the expected cross section limit for the $W H \rightarrow \ell \nu b \bar{b}$, $g g \rightarrow H \rightarrow W^{+} W^{-}$, and this analysis results [84].

\subsection{Conclusions}

We searched for the neutral Higgs boson production associated with the $W$ boson using high- $p_{T}$ isolated like-sign dilepton events in the CDF Run-II data corresponding to an integrated luminosity of $193.5 \mathrm{pb}^{-1}$. We first studied the background components in our base-line like-sign sample which was created by requiring the leading lepton $p_{T}$ $>20 \mathrm{GeV} / c$ and the second lepton $p_{T}>6 \mathrm{GeV} / c$, and confirmed that, overall, the entire sample was consistent with our background expectation. Based on the $S / \sqrt{B}$ calculation using signal Monte Carlo's and our background expectation, the signal region was then determined in the plane of the second lepton $p_{T}\left(p_{T 2}\right)$ versus the vector sum of $p_{T}$ 's of the two leptons $\left(p_{T 12}\right)$. The signal region was $p_{T 2}>16$ (18) $\mathrm{GeV} / c$ and $p_{T 12}>35 \mathrm{GeV} / c$ for the Higgs boson masses $<160 \mathrm{GeV} / c^{2}(>160$ $\left.\mathrm{GeV} / c^{2}\right)$. No event was found, while the total background was expected to be 0.95 \pm 0.61 (stat.) \pm 0.18 (syst.) events, the $110 \mathrm{GeV} / c^{2}$ bosophilic (fermiophobic) Higgs 
to be about 0.06 events assuming the same production cross section as the Standard Model Higgs boson, and the $160 \mathrm{GeV} / c^{2}$ Standard Model Higgs boson to be about 0.03 events. We set cross section upper limits $\sigma(W H) \times \operatorname{Br}(H \rightarrow W W)<12 \mathrm{pb}$ at the $95 \%$ C.L. for the $110 \mathrm{GeV} / c^{2}$ Higgs and $8 \mathrm{pb}$ for the $160 \mathrm{GeV} / c^{2}$ Higgs. This analysis was structured from simple techniques only: conventional isolation, high- $p_{T}$ lepton identification, and simple kinematical requirements to define the signal region. There were no signal-specific cuts such as missing- $E_{T}$ and other topological cuts. The present result therefore provided a conservative physics interpretation. 
CDF Run II Preliminary

\begin{tabular}{c|cccc}
\hline \hline Higgs boson mass $\left(\mathrm{GeV} / c^{2}\right)$ & 90 & 100 & 110 & 120 \\
\hline$W H$ & $17.5 \pm 4.8$ & $17.4 \pm 4.8$ & $16.1 \pm 4.8$ & $13.9 \pm 4.5$ \\
Observed $W H$ & 13.0 & 13.0 & 11.7 & 9.8 \\
$W H+Z H$ & $22.6 \pm 3.9$ & $20.2 \pm 4.6$ & $19.2 \pm 4.7$ & $19.9 \pm 4.7$ \\
Observed $W H+Z H$ & 19.9 & 16.1 & 14.8 & 15.7 \\
\hline Higgs boson mass $\left(\mathrm{GeV} / c^{2}\right)$ & 130 & 140 & 150 & 160 \\
\hline$W H$ & $13.1 \pm 4.4$ & $11.0 \pm 3.7$ & $11.1 \pm 3.7$ & $12.0 \pm 4.0$ \\
Observed $W H$ & 9.2 & 7.7 & 7.8 & 8.4 \\
$W H+Z H$ & $16.9 \pm 4.8$ & $16.6 \pm 4.9$ & $14.9 \pm 4.6$ & $15.9 \pm 4.8$ \\
Observed $W H+Z H$ & 12.4 & 12.1 & 10.7 & 11.5 \\
\hline Higgs boson mass $\left(\mathrm{GeV} / c^{2}\right)$ & 170 & 180 & 190 & 200 \\
\hline$W H$ & $10.9 \pm 3.7$ & $11.0 \pm 3.7$ & $10.0 \pm 3.5$ & $10.6 \pm 3.7$ \\
Observed $W H$ & 7.7 & 7.7 & 7.0 & 7.4 \\
$W H+Z H$ & $15.2 \pm 4.6$ & $14.0 \pm 4.5$ & $15.2 \pm 4.6$ & $13.7 \pm 4.4$ \\
Observed $W H+Z H$ & 10.9 & 10.0 & 11.0 & 9.7 \\
\hline \hline
\end{tabular}

Table 7.1: Expected limit and observed limit for each Higgs boson mass point. The error is taken from the RMS of limit distribution. 

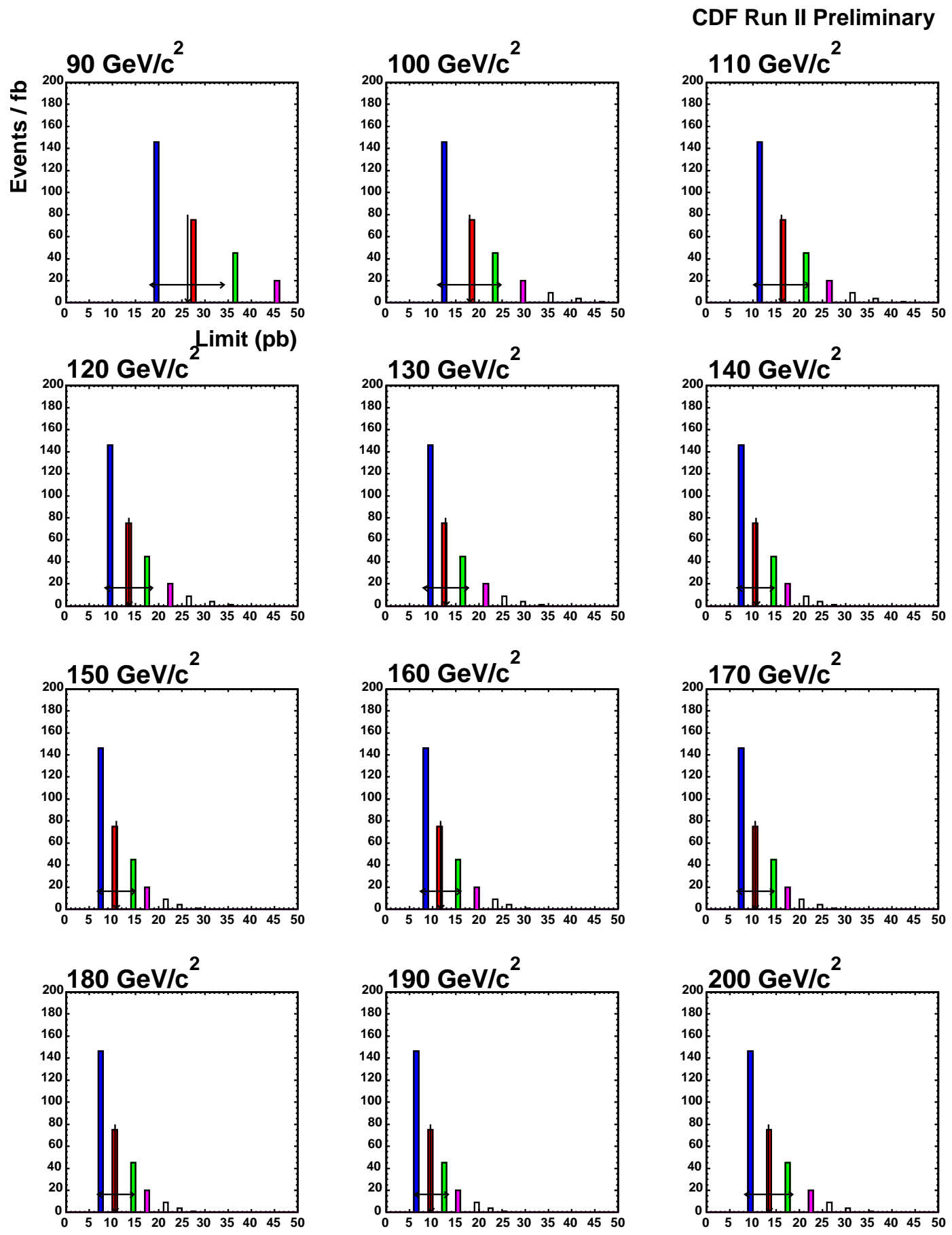

Figure 7.1: Upper limit on the cross section for each mass point from pseudoexperiments. Arrows show the mean and RMS. 

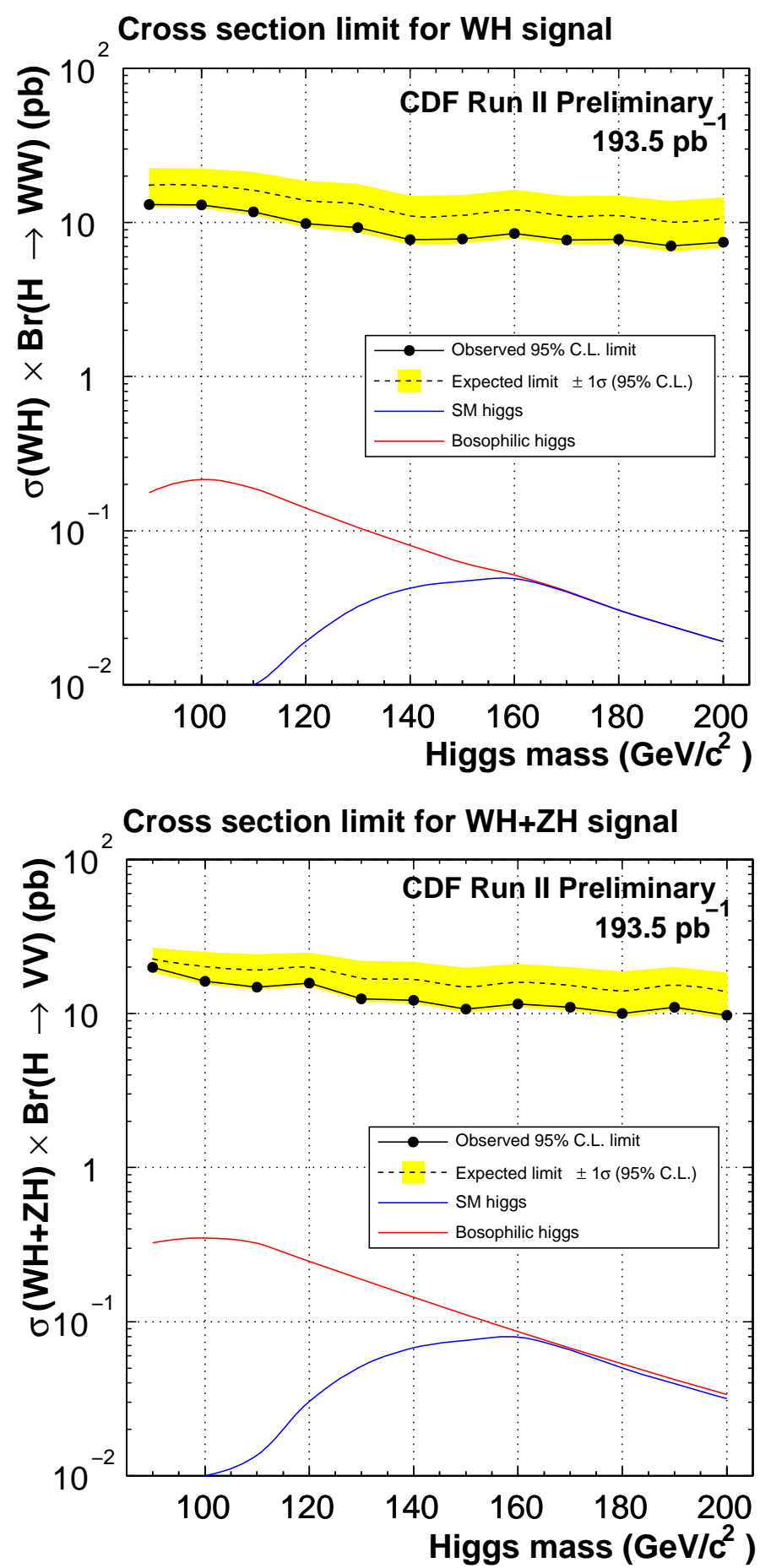

Figure 7.2: Upper (Lower) plot shows 95\% C.L. upper limits on the production cross section times branching fraction as a function of Higgs boson mass for the $W H(W H+$ $Z H)$ production, together with the cross sections of the Standard Model and of the benchmark scenario for the bosophilic Higgs boson. Results from pseudo-experiments for expected limits are also shown. 


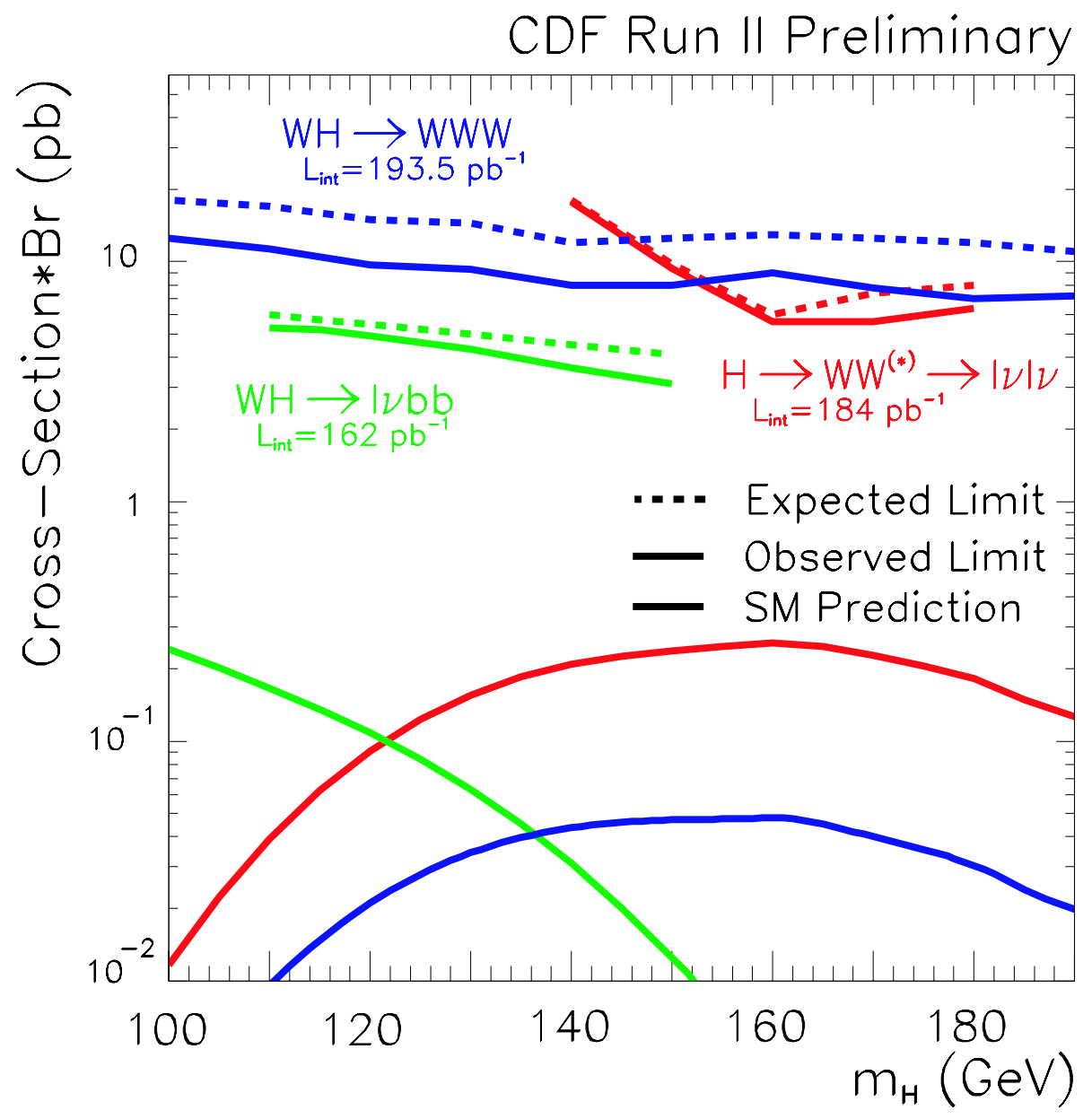

Figure 7.3: CDF Run II preliminary cross section limit for the Standard Model Higgs boson. 


\section{Bibliography}

[1] P. Renton, "Electroweak Interactions: Introduction to the Physics of Quarks and Leptons", Cambridge University Press (1990).

[2] L. Smolin, "How far are we from the quantum theory of gravity?", hep-th/0303185, (2003).

[3] K. Hagiwara et al.., Phys. Rev. D 66010001 (2002).

[4] R. K. Ellis, W. J. Stirling \& B. R. Webber. "QCD and Collider Physics". Cambridge University Press (1996).

[5] P. W. Higgs, "Broken symmetries, massless particles and gauge fields", Phys. Lett. 12 132-133 (1964).

[6] P. W. Higgs, "Spontaneous symmetry breakdown without massless bosons", Phys. Rev. 145 1156-1163 (1966).

[7] P. W. Higgs, "Broken symmetries and the masses of gauge bosons", Phys. Rev. Lett. 13 508-509 (1964).

[8] Gerard 't Hooft, "Renormalization of massless Yang-Mills fields", Nucl. Phys. B33 173-199 (1977).

[9] Gerard 't Hooft, "Renormalisable lagrangians for massive Yang-Mills fields", Nucl. Phys. B35 167-188 (1971).

[10] M. J. G. Veltman, "Perturbation theory of massive Yang-Mills fields", Nucl. Phys. B7 637-650 (1968).

[11] M. Carena, J. S. Conway, H. E. Haber, J. D. Hobbs, et al., hep-ph/0010338 (2000).

[12] The LEP Collaborations ALEPH, DELPHI, L3, OPAL, the LEP Electroweak Working Group and the SLD Heavy Flavour Group, "A Combination of Preliminary Electroweak and Constraints on the Standard Model", CERN-EP/2002-091 hep-ex/0212036 (2002). 
[13] The LEP Collaborations ALEPH, DELPHI, L3, OPAL, the LEP Electroweak Working Group, "Search for the Standard Model Higgs Boson at LEP", CERN$\mathrm{EP} / 2003-011$ (2003).

[14] T. Hambye and K. Riesselmann, Phys. Rev. D 557255 (1997).

[15] G. Altarelli and G. Isidori, Lett. B337 141 (1994); J. A. Casas, J. R. Espinosa and M. Quiros, Phys. Lett. B342 171 (1995); B382 374 (1996).

[16] K. Riesselmann, DESY-97-222 hep-ph/9711456 (1997).

[17] M. Spira, hep-ph/9711456, (1997).

[18] H. Fusaoka and Y. Koide, Phys. Rev. D57 3986 (1998).

[19] A. Djouadi, J. Kalinowski and M. Spira, Comput. Phys. Commun. 10856 (1998).

[20] A. Djouadi, J. Klinowski, and M. Spira, Z. Phys. C70 435 (1996); S. Moretti and W. J. Stirling, Phys. Lett. B347 291 (1995).

[21] T. G. Rizzo, Phys. Rev D 22389 (1980); W. -Y. Keung and W. J. Marciano, Phys. Rev. D 30248 (1984); R. N. Cahn, Rep. Prog. Phys. 52389 (1989).

[22] See e.g. , ATLAS Collaboration, "ATLAS Detector and Physics Performance: Technical Design Report, Volume 2", ATLAS TDR 15, CERN/LHCC 99-15 Chapter 19, (1999).

[23] A computer program library with cross-section fro all Higgs production processes relevant to the Tevatron Higgs search is available from http://people.web.psi.ch/spira/proglist.html .

[24] T. Han and S. Willenbrock, Phys. Lett. B273 167 (1991).

[25] S. Mrenna and C. P. Yuan, Phys. Lett. B416 200 (1998).

[26] M. Spira, Fortschr. Phys. 46203 (1998).

[27] R. K. Ellis and S. Veseli, Phys. Rev. D 60011501 (1999).

[28] H. Georgi, S. Glashow, M. Machacek, and D. V. Napnoulos, Phys. Rev. Lett. 40 692 (1978).

[29] A. Djouadi, M. Spira, and P. M. Zerwas, Phys. Lett. B264 440 (1991); S. Dawson, Nucl. Phys. B359 283 (1991). 
[30] D. Graudenz, M. Spira, and P. M. Zerwas, Phys. Rev. Lett. 701372 (1993); M. Spira, A. Djouadi, D. Graudenz, and P. M. Zerwas, Nucl. Phys. B453 17 (1995).

[31] C. Balazs and C. -P. Yuan, Phys. Lett. B478 192 (2000).

[32] C. Nelson, Phys. Rev. D 371220 (1988).

[33] M. Dittmar and H. Dreiner, Phys. Rev. D 55167 (1997).

[34] A. Stange, W. Marciano, and S. Willenbrock, Phys. Rev. D 491354 (1994).

[35] The ALEPH, DELPHI, L3, and OPAL Collaborations The LEP Higgs Working Group, "Searches for Higgs Bosons Decaying into Photons: Preliminary Combined Results Using LEP Data Collected at Energies up to 209 GeV", hep-ex/0107035, (2001).

[36] P. J. Wilson and H. J. Frisch, "Search for a Higgs Decaying to Two Photons", Phys. Rev. D64 092002 (2001).

[37] "Details about Fermilab's accelerators", http://www.fnal.gov//pub/inquiring/physics/accelerators/chainaccel.html.

[38] "Tevatron Run II handbook", http://www-bd.fnal.gov/lug/runII_handbook/RunII_index.html.

[39] F. Abe et al., Nucl. Instrum. Methods Phys. Res. Sect. A 271, 387 (1988); D. Amidei et al., Nucl. Instum. Methods Phys. Res. Sect. A 350, 73 (1994); F. Abe et al., Phys. Rev. D 52, 4784 (1995); P. Azzi et al., Nucl. Instrum. Methods Phys. Res. Sect. A 360, 137 (1995); The CDF collaboration, "The CDF II Detector Technical Design Report", Fermilab-Pub-96/390-E (1996).

[40] In the CDF coordinate system, $\theta$ is the polar angle with respect to the proton beam direction and $\phi$ is the azimuthal angle. The pseudorapidity is defined as $-\ln (\tan (\theta / 2))$. The transverse momentum of a particle is $p_{T}=p \sin \theta$. The transverse energy defined for calorimeter responses is similarly given by $E_{T}=E \sin \theta$. The missing transverse energy $\mathbb{E}_{T}$ is the vector pointing opposite the vector sum of transverse energies over all the calorimeter towers.

[41] T. Sjostrand, L. Lonnblad, and S. Mrenna, Comput. Phys. Commun. 135, 238 (2001).

[42] J. Cockcroft and E. Walton. Proc. Roy. Soc., 136:619, (1932). 
[43] W. K. H. Panofsky et al., Berkeley Proton Linear Accelerator, Rev. Sci. Instrum., 26:111, (1955).

[44] D. Mohl, G. Petrucci, L. Thorndahl, and Simon Van Der Meer, "Physics and Technique of Stochastic Cooling", Phys. Rept. 58:73-119 (1980).

[45] The CDF II Collaboration, "The CDF II Detector Technical Design Report", Fermilab-Pub-96, 390-E, (1996).

[46] A. Mukherjee and R. Wagner, "COT Central Outer Tracker", Public CDF6267, (2003).

[47] C. Green, J. Kowalkowsi, and M. Paterno, "CDF Simulation Framework Developer's Guide", CDF5369, (2000).

[48] H. Wenzel "Tracking in the SVX", CDF1790 (1998).

[49] M. Brozovic, A. T. Goshaw, and W. J. Robertson, "Jet Vertex Resolution in a Run Z Event Sample", CDF5698, (2001).

[50] M. Coca et al., "A First Look at Run II High $p_{T}$ Electrons", CDF5803, (2003).

[51] K. Yasuoka et al., Nuclear Instruments and Methods, A 267315 (1988).

[52] P. Tamburello, http://www-cdf.fnal.gov/internal/physics/top/r2leptons/etf/agenda/ 10_15_01.html

[53] J. Nielsen et al., "Trigger Efficiencies for High $E_{T}$ Electrons", CDF6234, (2002).

[54] J. D. Bellinger et al., "A Guide to Muon Reconstruction and Software for Run II", CDF5870, (2002).

[55] C. Hays et al., "The COT Pattern Recognition Algorithm and Offline Code", CDF6992, (2004).

[56] M. Karagoz Unel et al., "Search for New Particles in High Mass Dimuon Channel in CDF Run II Data", CDF6344, (2004).

[57] See http://www-cdf.fnal.gov/internal/physics/joint_physics/docs/mueff.pdf, (2004).

[58] M. Karagoz Unel, Thesis "Searches for new Physics Using High Mass Dimuons at the CDF II Experiment", CDF7296 (2004). 
[59] "Measurement of $Z$ and Drell-Yan Production Cross-Section Using Dimuons in $p \bar{p}$ Collisions at $\sqrt{s}=1.8 \mathrm{TeV}$ ", Fermilab-pub-98/280-E. CDF4092, (1998).

[60] P. de Barbaro, A. Bodek, E. Hayashi, K. Maeshima, C. Grosso-Pilcher, and M. Pillai, "A Search for $Z^{\prime}$ decaying to $\mu \mu$ using Run IA data and a first look at Run 1B data", CDF2910, (1994).

[61] W. Badgett, Ph. D Thesis, University of Michigan, "Measurement of $R(W / Z)$ from muons", (1992).

[62] P. Schlabach, "Probing the Central Calorimeter Energy Scale with Run0 Muons", CDF2896, (1994).

[63] F. Abe et al., Phys. Rev. Lett., 74 2900, (1995).

[64] V. Martin and L. Cerrito, "Muon Cuts and Efficiencies for 4.11 Analysis", CDF6825, (2004).

[65] Top group data of 4.11.1, http://www-cdf.fnal.gov/internal/physics/top/topdata/TopData_4111.html.

[66] D. Perkins, "Introduction to High Energy Physics", Addison Wesley Publishing Company, (2000).

[67] http://www-cdf.fnal.gov/internal/dqm/goodrun/good.html. We use the list of version 4.

[68] J. Nachtman M. Worcester D. Saltzberg, "Background Estimation and Verification for the Like-sign Dilepton Search", CDF6365, (2003).

[69] A. Taffard, "Run II Cosmic Ray tagger", CDF6100, (2003).

[70] H. Bachacou et al., "ttbar Event Selection and Detection Efficiency for Winter 2003 Lepton+Jets Analyses", CDF6084, (2003).

[71] H. Kobayashi, K. Yamamoto, Y. Seiya, "Base-Line Selection of Like-sign Dilepton Events from High- $p_{T}$ Inclusive Lepton Datasets in RUN II", CDF7260, (2004).

[72] J. Proudfoot, F. Ukegawa, A. B. Wicklund, "PHOTON CONVERSION BACKGROUND IN THE INCLUSIVE ELECTRON SAMPLE", CDF1297, (1990).

[73] C. Ferretti, http://cdfrh0.grid.umich.edu ${ }^{\sim} \quad$ claudiof/physics/Wjets/xsec/alpgen_xsec.html .

[74] D. Amidi et al., "Measurements of $W \rightarrow \mu \nu$ and $Z \rightarrow \mu \mu$ production cross sections and R using CDF Run II Data", CDF6681, (2004). 
[75] D. Amidi et al., "Measurements of $\sigma \times B(W \rightarrow e \nu), \sigma \times B(Z \rightarrow e e)$ and the ratio R using CDF Run II Data," CDF6711, (2004).

[76] B. Rossi, "High-Energy Particles", Prentice-Hall, (1952).

[77] H. Kobayashi, K. Yamamoto, Y. Seiya, "Background Estimation for High- $p_{T}$ Likesign Dilepton Analysis", CDF7261, (2004).

[78] W. K. Sakumoto A. Hocker, "Event $\left|z_{v t x}\right|<60 \mathrm{~cm}$ Cut Efficiency for Run II", CDF6331, (2003).

[79] V. Martin L. Cerrito, "Muon Cuts and Efficiencies for 4.11 Analyses", CDF6825, (2004).

[80] Y. K. Kim, J. Nielsen, L. Tompkins, G. Veramendi, "Trigger Efficiencies for High $p_{T}$ Electrons", CDF6234, (2004).

[81] CDF Statistics Committee - Software : "BAYES", See also the committee's web pages http://www-cdf.fnal.gov/physics/statistics/statistics_software.html.

[82] The CDF Collaboration, "Search for New Particle $X \rightarrow b \bar{b}$ Produced in Association with $W$ Bosons at $\sqrt{s}=1.96$ TeV", Public CDF7126, (2004).

[83] The CDF Collaboration, "Search for the Standard-Model Higgs Boson in the Decay Channel of $H \rightarrow W W \rightarrow \ell \nu \ell \nu$ with $184 \mathrm{pb}^{-1}$ of Run II Data at CDF", Public CDF7152, (2004).

[84] The CDF Collaboration, "Search for the $W H$ Production Using High- $p_{T}$ Isolated Like-Sign Dilepton Events in Run II", Public CDF7307, (2004). 